\title{
PASSIVE FLOW CONTROL OF ROTARY-WING AND FIXED- WING AIRCRAFT AIRFOILS VIA SURFACE-BASED TRAPPED VORTEX GENERATORS
}

\author{
By \\ Khider J. Al-Jaburi \\ M.Sc. Aeronautical Engineering \\ in partial fulfillment of the requirements for the degree of
}

A thesis submitted to the Faculty of Graduate Studies and Research

Doctor of Philosophy

in

Aerospace Engineering

Ottawa-Carleton Institute for Mechanical \& Aerospace Engineering

Department of Mechanical \& Aerospace Engineering

Carleton University

Ottawa, Ontario, Canada

Copyrights $@$ Khider J. Al-Jaburi

January 2019 
To the secret of existence and the source of life, who gave me all the love and compassion, my Mother.

To the one who sacrificed himselffor my happiness, who did his utmost to get me to this stage, who strived for my comfort, my Father.

To the ones who stood beside me, have been my inspiration and motivation to continue on, my Wife and Children.

To you all, I modestly dedicate my thesis. 


\begin{abstract}
A novel passive flow control concept for subsonic and transonic flows over 2D airfoils is proposed and examined via CFD. The control concept is based on the local modification of the airfoil geometry via a newly proposed Surface-based Trapped Vortex Generator (STVG) concept. This aims to reduce drag or to increase lift without deteriorating the original lift and/or drag characteristics of the airfoil, respectively. The benefits of such flow control technique were demonstrated for flow conditions representative of the flows seen on commercial jets in cruise or on helicopter main rotors in forward flight.
\end{abstract}

For transonic flows, a NACA 0012 airfoil exposed to a freestream of Mach 0.7 and $\operatorname{Re}=9 \times 10^{6}$ as well as a NASA SC(3)-0712(B) supercritical airfoil exposed to a freestream of Mach 0.78 and $\mathrm{Re}=30 \times 10^{6}$, were examined. The upper surface modifications demonstrated the ability to reduce the strength of the shockwave on the upper surface of the airfoil with only a small penalty in lift, yet, with increased lift-to-drag ratio. Lower surface modifications could significantly increase the lift-to-drag ratio for the full range of the investigated angles of attack. Moreover, the proposed geometries were investigated for a range of Mach number from 0.3 to 0.86 using NASA SC(3)-0712(B) supercritical airfoil. The modified airfoil outperforming the baseline airfoil at all freestreams.

For helicopter main rotor flows, three major contributions were made. First, the STVG control was applied to a NACA 0012 airfoil undergoing dynamic stall at constant freestream. Here the aim was to mitigate the negative effects of dynamic stall, i.e. for the reduction of peak negative pitching moment while not deteriorating significantly the original lift and drag characteristics. 2D CFD simulations of a NACA 0012 airfoil exposed to a freestream of Mach 0.3 and $\mathrm{Re}=3.76 \times 10^{6}$ and undergoing $\mathrm{a} 15^{\circ} \pm 10^{\circ}$ pitch oscillation 
with a reduced frequency of 0.101 were conducted. In case of the upper surface modifications, the best geometries could reduce the peak negative pitching moment by as much as $37-63 \%$, while sacrificing only $2-10 \%$ of peak lift and reducing drag by $14-38 \%$. On the other hand, the lower surface modifications demonstrated the ability to increase lift by $4-16 \%$ with only minor penalty in pitching moment and drag.

Second, a comprehensive methodology for simulating 2D (shock-induced) dynamic stall at fluctuating freestream was proposed in this work. 2D CFD simulation of a SC1095 airfoil exposed to a fluctuating freestream of Mach $0.537 \pm 0.205$ and $R e=$ $6.1 \times 10^{6}$ (based on the mean Mach number) and undergoing a $10^{\circ} \pm 10^{\circ}$ pitch oscillation with a frequency of $4.25 \mathrm{~Hz}$ was conducted. These conditions were representative of the flow experienced by a helicopter rotor blade section of the UH-60A helicopter in forward flight. The results suggest that the fluctuating freestream alters the dynamic stall mechanism documented for constant freestream in a major way, emphasizing that inclusion of this effect in the prediction of dynamic stall related rotor loads is imperative for rotor performance analysis and blades design.

Finally, the novel STVG passive flow control concept was investigated for controlling the flow in 2D shock-induced dynamic stall at fluctuating freestream. The SC1095 airfoil was exposed to the fluctuating freestreams mentioned above but this time with the STVG concept. Results showed that the best geometries could reduce the peak negative pitching moment by as much as $9-23 \%$ during the transonic phase of a cycle and by as much as $19-71 \%$ during the dynamic stall phase. Also, they are able to reduce peak drag by $8-20 \%$ for the transonic phase and by $15-44 \%$ in the dynamic stall phase. On the other hand, the lift-to-drag ratio was significantly increased by $3-28 \%$ per one rotor cycle. All the above advantages came at virtually no penalty in lift. 


\section{Preface}

The current thesis consists of Chapters $1-5$, which encompass in Chapter 1 the introduction and the motivation behind the research, in Chapter 2 a literature survey of the most relevant studies on flow control techniques, the methodological approach in

Chapter 3, and the results and analysis sections in Chapter 4. Chapter 5 presents the conclusions, along with the thesis overall contributions and some future research recommenation.

The main findings of the thesis have been disseminated in a number of scientific publications (some of them still under review), which full list is provided below. The first three $[A-C]$ are conference papers while the next four $[D-G]$ are journal papers. They are listed in chronological order.

[A] Al-Jaburi K., Feszty D., "Passive Flow Control of Dynamic Stall via Surface-based Trapped Vortex Generators", $73^{\text {rd }}$ Annual Forum of the American Helicopter Society, Fort Worth, Texas, May 9-11, 2017.

[B] Jee D., Al-Jaburi K. and Feszty D., "Impact of variable freestream 2D CFD dynamic stall data on helicopter forward flight performance and prediction", $36^{\text {th }}$ Aeronautic Conference, AERO 2017 conference, CASI (Canadian Aeronautics and Space Institute), Toronto, ON, Canada, May 16 - 18, 2017.

[C] Jee D., Al-Jaburi K. and Feszty D., "Investigating the impact of using CFD generated unsteady Mach number dynamic stall data for numerical rotor analysis of helicopter forward flight", 35 ${ }^{\text {th }}$ AIAA Applied Aerodynamics Conference, AIAA AVIATION Forum, Denver, Colorado, June 5-9, 2017.

[D] Al-Jaburi, K. J., and Feszty, D., "Passive Flow Control of Dynamic Stall via Surfacebased Trapped Vortex Generators," Journal of the American Helicopter Society, Vol. 63, No. 3, 2018, pp. 1-14.

doi: https://doi.org/10.4050/JAHS.63.032005 
[E] Al-Jaburi, K. J., Feszty, D., and Nitzsche, F. "A Methodology for Simulating 2D Shock-induced Dynamic Stall at Flight Test-based Fluctuating Freestream," Chinese Journal of Aeronautics (to be published), 2018.

[F] Al-Jaburi, K. J., and Feszty, D., "Fixed and Rotary Wing Transonic Aerodynamic Improvement via Surface-based Trapped Vortex Generators," Journal of Aerospace Engineering (under review), 2018.

[G] Al-Jaburi, K. J., Feszty, D., and Nitzsche, F. "Passive Flow Control of 2D Shock induced Dynamic Stall at Flight Test-based Fluctuating Freestream," submitted to the Journal of Aerospace Science and Technology, 2018.

Part of the materials discussed in Chapter 2, Section 2.6; Chapter 3, Section 3.4;

Chapter 4, Subsection 4.4.1; and Chapter 5, Subsection 5.1.2 were copyrighted materials. They were published in the American Helicopter Society (AHS) peer-reviewed journal paper [D] or [87] in this work. Permission to reprint the texts, figures and tables from paper [D] in the sections indicated above had been obtained from the AHS publisher, as designated by the letters associated with the thesis document. Readers who wish to cite from the indicated copyrighted materials are required to cite paper [D] itself as per the citations listed before.

The rest of the thesis is not copyrighted materials as yet; hence, readers who wish to cite any section from the thesis (other than those from [D]) are advised to cite the current thesis itself.

Khider Al-Jaburi was responsible for the main idea, the designs, the numerical analyses of the present research, and prepared all manuscripts. Dr. Daniel Feszty and Professor Fred Nitzsche provided thesis supervisions and feedbacks on the draft manuscripts. 


\section{Acknowledgements}

First, I would like to give my earnest thanks and appreciation to my parents for their unconditional support, reassurance, love and encouragements during my endeavors to complete this research. Thank you for always being there for me when I need you even when we are miles apart. Without you, this research would not have been possible.

My sincere gratitude goes to Dr. Daniel Feszty. I still remember, it was early in the Winter of 2014 term when I first approached his office with my idea of using the airfoil geometry of the paper airplane in a scientific research. A research involves two of the most complicated aerodynamic phenomena. He was very open-minded and supportive. He responded with a smile and thoughtfulness, "why not, let us do it". It was more than what a young researcher would have needed to peruse his goals. From that day forward, Dr. Feszty has done his utmost to help me achieve my research goals and bring them to life. I also wish to gratefully acknowledge Professor Fred Nitzsche for his co-supervision and overseeing the last stages of the research.

To my brother and sisters, thank you for your love, encouragement, and support to accomplish this huge task. My profound thanks go to my children Baylasan, Layan, and Leen for their unwavering understanding at a young age that their father had to work so hard and be absent from them so much. Forgive me for not often playing with you and spend enough time with you. Thank you for inspiring me to be the best father I can ever be for you. And most of all, I graciously thank my wife Zainab. Dear, I cannot begin to express my gratitude for all your love and support, and for all you have sacrificed willingly without any hesitations to help me through all the stages of this research. 


\section{Table of Contents}

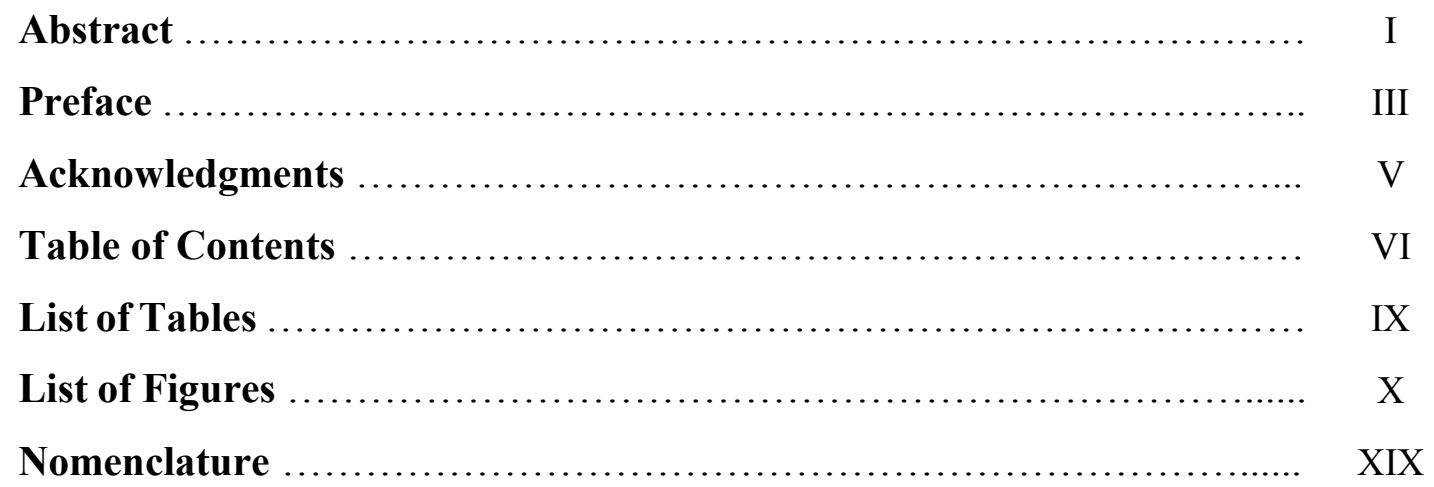

1 Introduction 1

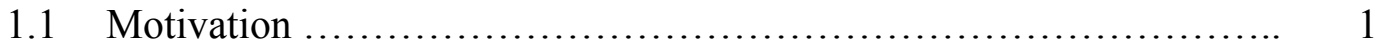

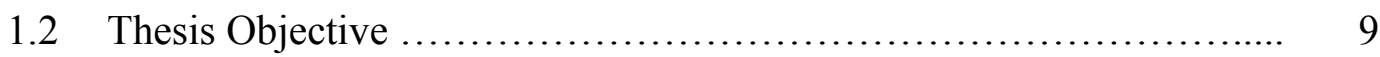

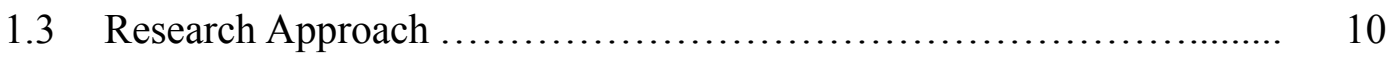

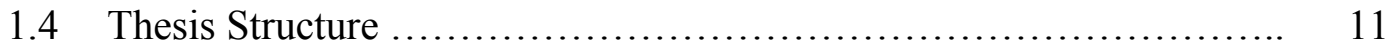

2 Background and Literature Review $\quad 12$

2.1 Chapter Overview .......................................... 12

2.2 Backward Facing Step and flow Control Methods.................... 15

2.3 Airfoils with Backward Facing Step on the Surface ................. 21

2.3.1 Unconventional Airfoils: Kline-Fogleman (KF) Airfoils...... 21

2.3.2 Conventional Airfoils with Backward Facing Step............ 23

2.4 The Vortex as a Mean of Airfoil Flow Control ....................... 27

2.5 Flow Control Methods for Rotorcraft Airfoils ........................ 33

2.6 Flow Control Methods for Airfoil Dynamic Stall ................... 37

2.7 The Novelty of Present Research .............................. 40

3 Computational Setup and Procedure $\quad 42$

$3.1 \quad$ Chapter Overview $\ldots \ldots \ldots \ldots \ldots \ldots \ldots \ldots \ldots \ldots \ldots \ldots \ldots \ldots \ldots \ldots \ldots, \quad 42$

3.2 Numerical Method ........................................ 43

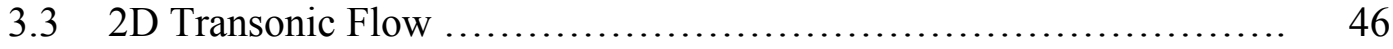

3.3.1 Test Cases ........................................ 46 
3.3.2 Computational Domain ................................. 46

3.3.3 Mesh Generation ................................................. 49

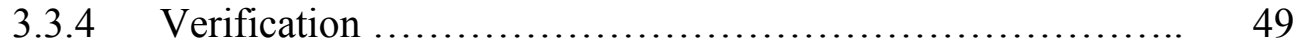

3.3.5 Validations .............................................. 51

3.3.6 Simulation Precautions ..................................... 52

3.4 2D Constant Freestream Dynamic Stall ............................ 57

3.4.1 Test Cases ................................................ 57

3.4.2 Computational Domain ................................. 57

3.4.3 Mesh Generation ........................................... 60

3.4.4 Initialization ........................................ 60

3.4.5 Verification ........................................... 62

3.4.6 Validations ........................................... 62

3.5 2D Fluctuating Freestream Dynamic Stall ......................... 65

3.5.1 Test Cases ............................................... 65

3.5.2 Computational Domain ................................ 67

3.5.3 Computational Domain Reconfiguration .................... 68

3.5.4 Computational Time Delay .............................. 70

3.5.5 Mesh Generation ............................................ 71

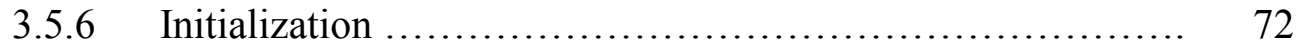

3.5.7 Verification .......................................... 73

3.5.8 Validations .......................................... 74

$4 \quad$ Results and Discussions $\quad \mathbf{8 0}$

$4.1 \quad$ Chapter Overview ............................................... $\quad 80$

4.2 Passive Flow Control Geometries ................................. 81

4.3 Passive Flow Control for 2D Transonic Aerodynamic Improvement .... 83

4.3.1 NACA 0012 Airfoil (Test Case 1) ........................... 85

4.3.1.1 NACA 0012 with $\mathrm{G}^{1} \mathrm{~L} 1$ Modification ............... 88

4.3.1.2 NACA 0012 with $\mathrm{G}^{3} \mathrm{~L} 6$ Modification ............... 91

4.3.1.3 NACA 0012 with $\mathrm{G}^{4}$ LTV1 Modification ............. 94

4.3.2 NASA SC(3)-0712(B) Supercritical Airfoil (Test Case 2) ...... 98

4.3.2.1 NASA SC(3)-0712(B) with $\mathrm{G}^{2} \mathrm{U} 3$ Modification ...... 98 
4.3.2.2 NASA SC(3)-0712(B) with G ${ }^{4}$ LTV1i Modification 102

4.3.3 Passive Flow Control Robustness Test ..................... 105

4.4 Passive Flow Control for Mitigation of 2D Dynamic Stall ............. 106

4.4.1 Constant Freestream Dynamic Stall ........................ 106

4.4.1.1 NACA 0012 with $\mathrm{G}^{1} \mathrm{U} 2$ Modification .............. 109

4.4.1.2 NACA 0012 with $\mathrm{G}^{3} \mathrm{U} 2$ Modification .............. 114

4.4.1.3 NACA 0012 with $\mathrm{G}^{2} \mathrm{~L} 1$ Modification ............... 119

4.4.2 Fluctuating Freestream Dynamic Stall ..................... 124

4.4.3 Fluctuating Freestream Dynamic Stall Control ............... 139

4.4.3.1 SC1095 with $\mathrm{G}^{3} \mathrm{U} 2$ Modification ................... 143

4.4.3.2 SC1095 with $\mathrm{G}^{2} \mathrm{~L} 1 \mathrm{Modification}$.................. 150

5 Conclusions, Contributions and Future Work 157

5.1 Conclusions ................................................... 157

5.1.1 Transonic Flow Aerodynamic Improvement ................ 157

5.1.2 Constant Freestream Dynamic Stall Mitigation ................ 159

5.1.3 Methodology to Investigate Shock-induced Dynamic Stall ..... 160

5.1.4 Shock-induced Dynamic Stall Mitigation ................... 161

5.2 Thesis Contributions ............................................ 162

$5.3 \quad$ Future Work ................................................. 165

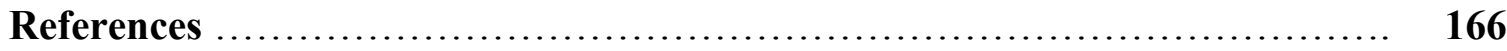

Appendix A. FLUENT Input Files ..................................... 182

Appendix B. Baseline Airfoils Geometries Coordinates ..................... 185

Appendix C. The Permission to Reproduce Copyrighted Materials ........... 196 


\section{List of Tables}

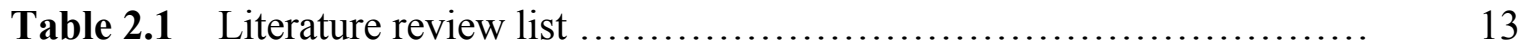

Table 2.1 Literature review list (Continued) …......................... 14

Table 2.2 Results summary from Al-Jaburi [22] experimental study ............. 27

Table 2.3 Summary of prior work on fluctuating freestream dynamic stall ...... 39

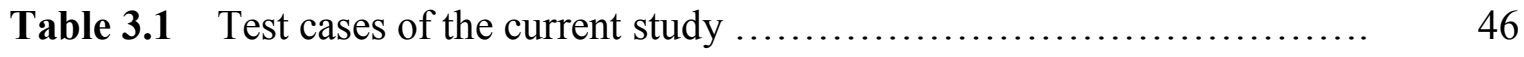

Table 3.2 Simulation parameters and conditions ............................ 65

Table 3.3 CFD literatures for fluctuating freestream dynamic stall in forward

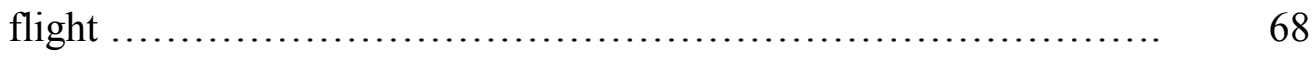

Table 4.1 Geometric specifications of the studied passive flow control .......... 84

Table 4.2 Results summary of the modified NACA 0012 airfoil ................ 87

Table 4.3 Results summary of the modified NACA 0012 airfoil ................ 108

Table 4.4 Summary of the CFD simulation results for the SC1095 at $f=4.25$ $\mathrm{Hz}, \operatorname{Re}=6.1 \times 10^{6}, M=0.537 \pm 0.205$ and $\alpha=10^{\circ} \pm 10^{\circ}$ 


\section{List of Figures}

Figure 1.1 Development of supersonic flow over an airfoil .................. 3

Figure 1.2

Lift, drag and pitching moment on a fixed-wing aircraft during

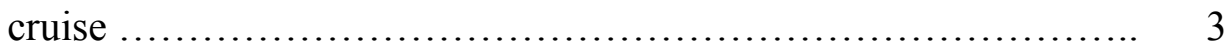

Figure 1.3 Top view of the main rotor disk and the velocity distribution [1] .... 4

Figure 1.4 Transonic and dynamic stall regions in forward flight .............. 5

Figure 1.5 Kline- Fogleman airfoil (a) and Fertis airfoil (b). Reproduced from

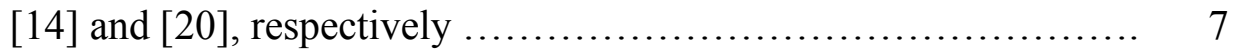

Figure 2.1 Flow over Backward Facing Step (reproduced from Paul [4]) ....... 15

Figure 2.2 Velocity profile distribution along Backward Facing Step

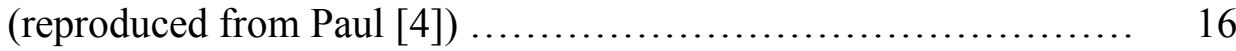

Figure 2.3 Kline-Fogleman Airfoil (reproduced from Kline et al. [14]) ......... 21

Figure 2.4 Kline-Fogleman Airfoil, $2^{\text {nd }}$ patent (reproduced from Kline et al.

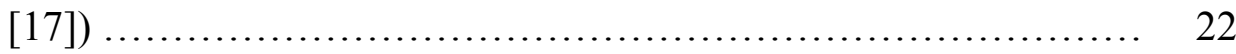

Figure 2.5 KFm family for RC airplanes (reproduced from [18]) ............. 23

Figure 2.6 Fertis-Smith airfoils (reproduced from Fertis et al. [20]) ............ 24

Figure 2.7 Fertis stepped airfoils with results (reproduced from Fertis [20]) ... 25

Figure 2.8 Baseline airfoil NACA 2415 and the airfoil with co-flow jet slot (reproduced from Zha and Paxton [26]) ....................... 28

Figure 2.9 Computational mesh of the synthetic jet inside the airfoil body (reproduced from You and Moin [27]) ......................... 29

Figure 2.10 Geometry of a leading-edge array of air-jet vortex generators with illustration of the physics of longitudinal vortex formation (reproduced from Prince et al. [30]) ......................... 30

Figure 2.11 Gurney flap (reproduced from Roedts [38]) ...................... 34

Figure 3.1 FLUENT control volume definition (reproduced from [80]) ........ 43 
Figure 3.2 Computational domain. (a) refined mesh area inside the domain (baseline airfoil) (b) refined mesh area inside the domain (modified airfoil) (c) mesh density of the surface modification (d) inflation layers and cell distribution around airfoil surface

Figure 3.3 Effects of distance to the far-field boundary with and without vortex correction for a NACA 0012 airfoil at $M=0.8, A o A=1.25^{\circ}$ (reproduced from [91])

Figure 3.4 Grid convergence analyses. Test Case 1. (a) NACA0012 airfoil, (b) NACA0012 airfoil with $\mathrm{G}^{3} \mathrm{~L} 6$ modification, $M=0.7$ and $R e=$ $9 \times 10^{6}$, angles of attack $4.74^{\circ}$ 50

Figure 3.5 Validation of Test Case 1, NACA0012 airfoil, $M=0.7$ and $R e=$ $9 \times 10^{6}$. First raw illustrates the aerodynamic results. Second raw illustrates the coefficient of the pressure distribution at angles of attack $1.5^{\circ}$ and $4.74^{\circ}$, respectively

Figure 3.6 Validation of Test Case 2, NASA SC(3)-0712(B) airfoil, $M=0.78$ and $R e=30 \times 10^{6}$, First raw illustrates the aerodynamic results. Second raw illustrates the coefficient of the pressure distribution at angles of attack $0.14^{\circ}$ and $1.04^{\circ}$, respectively

Figure 3.7 Computational domain and the mesh levels used in the transonic flow over rectangular cavity

Figure 3.8 Verification and validation of one of [8] test Cases, $M=0.8$ and $R e / m=1.55 \times 10^{7}$

Figure 3.9 NACA 0012 with $\mathrm{G}^{3} \mathrm{~L} 6$ modification 55

Figure 3.10 Time-independence study at the monitor points for Test Case 1, NACA 0012 with $\mathrm{G}^{3} \mathrm{~L} 6$ Modification at $A o A=3.01^{\circ}$ (first row) and $A o A=4.74^{\circ}$ (second row), at $M=0.7$ and $R e=9 \times 10^{6}$

Figure 3.11 Computational domain sketch, the "black" boundaries represent the pressure far field and the "gray" boundaries are the mesh interface .

Figure 3.12 Domain design study results representation using drag coefficient, $($ SST $k-\omega)$. NACA 0012, $M=0.3, R e=3.76 \times 10^{6}$ and $\alpha=15^{\circ} \pm 10^{\circ}$.. 
Figure 3.13 (a) Computational domain mesh, (b) refined mesh area inside the rotating domain and (c) inflation layers and cell distribution around airfoil surface

Figure 3.14 Time history of the aerodynamic loads for steady freestream dynamic stall simulation (SST $k-\omega)$. NACA 0012, $M=0.3, R e=$ $3.76 \times 10^{6}$ and $\alpha=15^{\circ} \pm 10^{\circ}$. Note the lack of initial transient, i.e. the periodicity of the curves immediately in the $1^{\text {st }}$ cycle after start-up ..

Figure 3.15 Verification tests, (a) grid-dependence, (b) time step dependence, (c) CFL number and (d) residual dependence. All using (SA). NACA $0012, M=0.3, R e=3.76 \times 10^{6}$ and $\alpha=15^{\circ} \pm 10^{\circ}$

Figure 3.16 Validation of the simulation results for (a) SA turbulence model and (b) SST $k-\omega$ turbulence model. NACA 0012, $M=0.3, R e=$ $3.76 \times 10^{6}$ and $\alpha=15^{\circ} \pm 10^{\circ}$

Figure 3.17 CAMRAD II vs Sinusoid AoA variation for UH-60A. Forward $\mathrm{M}=$ $0.205, \mathrm{r} / \mathrm{R}=0.865$ and advance ratio $\mu=0.33$ [71]

Figure 3.18 Freestream Mach number fluctuation versus angle of attack oscillation as a function of the azimuth angle

Figure 3.19 Computational domain sketch after the reconfiguration, the "black" lines represent the inlet and outlet boundaries with inlet to the left, the "dashed" lines were set to free-slip boundaries and the "gray" boundaries are the mesh interface

Figure 3.20 Mach number at the inlet and airfoil location (left) and the final Mach-AoA formulation used in the simulation (right)

Figure 3.21 Magnified images of the mesh used in dynamic stall simulation under fluctuating freestream

Figure 3.22 Time history of the aerodynamic loads for fluctuating freestream dynamic stall simulation. SC1095, $M=0.537 \pm 0.205, R e=6.1 \times 10^{6}$ and $\alpha=10^{\circ} \pm 10^{\circ}$

Figure 3.23 Results of the grid dependent study. SC1095, Frequency $=4.25 \mathrm{~Hz}$, $R e=6.1 \times 10^{6}, M=0.537 \pm 0.205$ and $\alpha=10^{\circ} \pm 10^{\circ}$ 74

Figure 3.24 Transonic flow validation. SC1095 airfoil, $R e=6.65 \times 10^{6}, M=0.8$. 75 
Figure 3.25 Validation of the fluctuating freestream conditions at the airfoil leading edge with the experiments of [106]. $R e=3 \times 10^{6}, \Delta \varphi=13.3^{\circ}$, $k=0.05, \alpha=8.5 \pm 13$ and $M=0.4 \pm 0.08$

Figure 3.26 Constant freestream (CFS) dynamic stall validated with [106]. SSCA09 airfoil, $\operatorname{Re}=3 \times 10^{6}, k=0.05, \alpha=8.5 \pm 13$ and $M=0.4 \ldots \ldots$

Figure 3.27 Fluctuating freestream (FFS) dynamic stall validated with [106].

SSC-A09 airfoil, $R e=3 \times 10^{6}, \Delta \varphi=13.3^{\circ}, k=0.05, \alpha=8.5 \pm 13$ and $M$ $=0.4 \pm 0.08$

Figure 4.1 Schematic of an airfoil with the geometric specifications of the BFS [22]

Figure 4.2 Airfoils with the passive geometries investigated in the study

Figure 4.3 Geometry modifications on a NACA 0012 and NASA SC(3)0712(B) airfoils 85

Figure 4.4 Aerodynamic loads of $\mathrm{G}^{1} \mathrm{~L} 1$ airfoil, $M=0.7$ and $R e=9 \times 10^{6} \ldots \ldots \ldots$ 89

Figure 4.5 Pressure coefficients of $\mathrm{G}^{1} \mathrm{~L} 1$ airfoil, $M=0.7$ and $R e=9 \times 10^{6}, \mathrm{AoA}$ $=2.5^{\circ}$

Figure 4.6 Mach number contours, baseline airfoil (left), $\mathrm{G}^{1} \mathrm{~L} 1$ airfoil (right), $M=0.7$ and $R e=9 \times 10^{6}, A o A=2.5^{\circ}$

Figure 4.7 Skin friction coefficient (x-component) for the baseline airfoil and the airfoil with $\mathrm{G}^{1} \mathrm{~L} 1$ modification, at $M=0.7$ and $R e=9 \times 10^{6}, C_{l}=$ 0.66

Figure 4.8 Aerodynamic loads of $\mathrm{G}^{3} \mathrm{~L} 6$ airfoil, $M=0.7$ and $R e=9 \times 10^{6}$ 92

Figure 4.9 Pressure coefficients of $\mathrm{G}^{3} \mathrm{~L} 6$ airfoil, $M=0.7$ and $R e=9 \times 10^{6}, \mathrm{AoA}$ $=2.5^{\circ}$

Figure 4.10 Mach number contours, baseline airfoil (left), $\mathrm{G}^{3} \mathrm{~L} 6$ airfoil (right), $M=0.7$ and $R e=9 \times 10^{6}, A o A=2.5^{\circ}$

Figure 4.11 Skin friction coefficient (x-component) for the baseline airfoil and the airfoil with $\mathrm{G}^{3} \mathrm{~L} 6$ modification, at $M=0.7$ and $R e=9 \times 10^{6}, C_{l}=$ 0.66

Figure 4.12 The $\mathrm{G}^{4} \mathrm{LTV} 1$ airfoil leading edge vortex, $M=0.7$ and $R e=9 \times 10^{6}$, $A o A=5^{\circ}$ 95

Figure 4.13 Aerodynamic loads of $\mathrm{G}^{4} \mathrm{LTV} 1$ airfoil, $M=0.7$ and $R e=9 \times 10^{6} \ldots . \quad 96$ 
Figure 4.14 Pressure coefficients of $\mathrm{G}^{4} \mathrm{LTV} 1$ airfoil, $M=0.7$ and $R e=9 \times 10^{6}$, $A o A=2.5^{\circ}$

Figure 4.15 Mach number contours, baseline airfoil (left), $\mathrm{G}^{4}$ LTV1 airfoil (right), $M=0.7$ and $R e=9 \times 10^{6}, A o A=2.5^{\circ}$

Figure 4.16 Skin friction coefficient (x-component) for the baseline airfoil and the airfoil with $\mathrm{G}^{4} \mathrm{LTV} 1$ modification, at $M=0.7$ and $R e=9 \times 10^{6}$, $C_{l}=0.66$

Figure 4.17 Aerodynamic loads for the NASA SC(3)-0712(B) airfoil with a $\mathrm{G}^{2} \mathrm{U} 3$ modification, $M=0.78$ and $R e=30 \times 10^{6}$ 100

Figure 4.18 Pressure coefficients for the NASA SC(3)-0712(B) airfoil with a $\mathrm{G}^{2} \mathrm{U} 3$ modification, $M=0.78$ and $R e=30 \times 10^{6}, A o A=2.5^{\circ} \ldots \ldots \ldots$

Figure 4.19 Mach number contours, NASA SC(3)-0712(B) baseline airfoil (left), with a $\mathrm{G}^{2} \mathrm{U} 3$ modification (right), $M=0.78$ and $R e=30 \times 10^{6}$, $A o A=2.5^{\circ}$

Figure 4.20 Aerodynamic loads for the NASA SC(3)-0712(B) airfoil with a $\mathrm{G}^{4}$ LTV1i modification, $M=0.78$ and $R e=30 \times 10^{6}$

Figure 4.21 Pressure coefficients for the NASA SC(3)-0712(B) airfoil with a $\mathrm{G}^{4}$ LTV1i modification, $M=0.78$ and $R e=30 \times 10^{6}, A o A=2.5^{\circ} \ldots \ldots$ 104

Figure 4.22 Mach number contours, NASA SC(3)-0712(B) baseline airfoil (left), with a $\mathrm{G}^{4} \mathrm{LTV} 1 \mathrm{i}$ modification (right), $M=0.78$ and $R e=$ $30 \times 10^{6}, A o A=2.5^{\circ}$ 104

Figure 4.23 Drag-Mach number graphs for the NASA SC(3)-0712(B) supercritical airfoil compared to $\mathrm{G}^{2} \mathrm{U} 3$ and $\mathrm{G}^{4} \mathrm{LTV} 1 \mathrm{i}$ modification at $A o A=2.5^{\circ}$ 106

Figure 4.24 NACA 0012 airfoil with the selected modifications applied 109

Figure 4.25 Aerodynamic loads for the $\mathrm{G}^{1} \mathrm{U} 2$ airfoil with the selected azimuthal positions. $M=0.3, \operatorname{Re}=3.76 \times 10^{6}$ and $\alpha=15^{\circ} \pm 10^{\circ}$

Figure 4.26 Pressure coefficient, instantaneous streamlines and velocity contours for the NACA 0012 airfoil and the $\mathrm{G}^{1} \mathrm{U} 2$ airfoil. $M=0.3$, $\operatorname{Re}=3.76 \times 10^{6}$ and $\alpha=15^{\circ} \pm 10^{\circ}$. 
Figure 4.26 (Continued) Pressure coefficient, instantaneous streamlines and velocity contours for the NACA 0012 airfoil and the $\mathrm{G}^{1} \mathrm{U} 2$ airfoil. $M=0.3, \operatorname{Re}=3.76 \times 10^{6}$ and $\alpha=15^{\circ} \pm 10^{\circ}$

Figure 4.26 (Continued) Pressure coefficient, instantaneous streamlines and velocity contours for the NACA 0012 airfoil and the $\mathrm{G}^{1} \mathrm{U} 2$ airfoil. $M=0.3, \operatorname{Re}=3.76 \times 10^{6}$ and $\alpha=15^{\circ} \pm 10^{\circ}$

Figure 4.27 Aerodynamic loads for the $\mathrm{G}^{3} \mathrm{U} 2$ airfoil with the selected azimuthal positions. $M=0.3, \operatorname{Re}=3.76 \times 10^{6}$ and $\alpha=15^{\circ} \pm 10^{\circ}$

Figure 4.28 Pressure coefficient, instantaneous streamlines and velocity contours for the NACA 0012 airfoil and the $\mathrm{G}^{3} \mathrm{U} 2$ airfoil. $M=0.3$, $R e=3.76 \times 10^{6}$ and $\alpha=15^{\circ} \pm 10^{\circ}$

Figure 4.28 (Continued) Pressure coefficient, instantaneous streamlines and velocity contours for the NACA 0012 airfoil and the $\mathrm{G}^{3} \mathrm{U} 2$ airfoil. $M=0.3, \operatorname{Re}=3.76 \times 10^{6}$ and $\alpha=15^{\circ} \pm 10^{\circ}$

Figure 4.28 (Continued) Pressure coefficient, instantaneous streamlines and velocity contours for the NACA 0012 airfoil and the $\mathrm{G}^{3} \mathrm{U} 2$ airfoil. $M=0.3, \operatorname{Re}=3.76 \times 10^{6}$ and $\alpha=15^{\circ} \pm 10^{\circ}$

Figure 4.29 Aerodynamic loads for the $G^{2} L 1$ airfoil with the selected azimuthal positions. $M=0.3, \operatorname{Re}=3.76 \times 10^{6}$ and $\alpha=15^{\circ} \pm 10^{\circ}$

Figure 4.30 Pressure coefficient, instantaneous streamlines and velocity contours for the NACA 0012 airfoil and the $\mathrm{G}^{2} \mathrm{~L} 1$ airfoil. $M=0.3$, $R e=3.76 \times 10^{6}$ and $\alpha=15^{\circ} \pm 10^{\circ}$

Figure 4.30 (Continued) Pressure coefficient, instantaneous streamlines and velocity contours for the NACA 0012 airfoil and the $\mathrm{G}^{2} \mathrm{~L} 1$ airfoil. $M=0.3, \quad R e=3.76 \times 10^{6}$ and $\alpha=15^{\circ} \pm 10^{\circ}$

Figure 4.30 (Continued) Pressure coefficient, instantaneous streamlines and velocity contours for the NACA 0012 airfoil and the $\mathrm{G}^{2} \mathrm{~L} 1$ airfoil. $M=0.3, \quad \operatorname{Re}=3.76 \times 10^{6}$ and $\alpha=15^{\circ} \pm 10^{\circ}$ 124

Figure 4.31 CFS $(M=0.4)$ vs. FFS $(M=0.4 \pm 0.08)$ dynamic stall of [106] experiment. SSC-A09 airfoil, $R e=3 \times 10^{6}, k=0.05, \alpha=8.5^{\circ} \pm 13^{\circ} \ldots \ldots$ 
Figure 4.32 CFS $(M=0.4)$ vs. FFS $(M=0.4 \pm 0.08)$ dynamic stall. Loads vs. coefficients. Scaled using the mean values vs. fluctuating values. Case study of [106] experiment. SSC-A09, $R e=3 \times 10^{6}, k=0.05, \alpha=$ $8.5 \pm 13$

Figure 4.33 CFS (left) vs. FFS (right) dynamic stall. White dashed-line represents maximum vorticity. Case study of [106] experiment. SSC-A09 airfoil, $R e=3 \times 10^{6}, k=0.05, \alpha=15.14^{\circ}$ and $M=0.4 \pm 0.08$

Figure 4.34 Compressible constant freestream (CFS) dynamic stall. SC1095, Re $=6.1 \times 10^{6}, f=4.25 \mathrm{~Hz}, \alpha=10 \pm 10$ and $M=0.537$

Figure 4.35 Top view sketch of UH-60A main rotor disk showing Mach number distribution along the blade in a forward flight phase according to Table 3.2, in Chapter 3

Figure 4.36 Comparison of aerodynamic loads at Fluctuating Freestream (FFS) dynamic stall, $(M=0.537 \pm 0.205)$ and at Constant Freestream (CFS) dynamic stall $(M=0.537)$ with the selected azimuthal positions. $\mathrm{SC} 1095, \operatorname{Re}=6.1 \times 10^{6}, f=4.25 \mathrm{~Hz}$, and $\alpha=10^{\circ} \pm 10^{\circ}$

Figure 4.37 Mach contours and instantaneous vorticities. SC1095 airfoil, $M=$ 0.537 (left) and $0.537 \pm 0.205$ (right), $R e=6.1 \times 10^{6}, f=4.25 \mathrm{~Hz}$ and $\alpha=10^{\circ} \pm 10^{\circ}$

Figure 4.37 (Continued) Mach contours and instantaneous vorticities. SC1095 airfoil, $M=0.537$ (left) and $0.537 \pm 0.205$ (right), $R e=6.1 \times 10^{6}, f=$ $4.25 \mathrm{~Hz}$ and $\alpha=10^{\circ} \pm 10^{\circ}$

Figure 4.37 (Continued) Mach contours and instantaneous vorticities. SC1095 airfoil, $M=0.537$ (left) and $0.537 \pm 0.205$ (right), $R e=6.1 \times 10^{6}, f=$ $4.25 \mathrm{~Hz}$ and $\alpha=10^{\circ} \pm 10^{\circ}$

Figure 4.37 (Continued) Mach contours and instantaneous vorticities. SC1095 airfoil, $M=0.537$ (left) and $0.537 \pm 0.205$ (right), $R e=6.1 \times 10^{6}, f=$ $4.25 \mathrm{~Hz}$ and $\alpha=10^{\circ} \pm 10^{\circ}$

Figure 4.38 UH-60A main rotor blade and SC1095 airfoil [111] 139

Figure 4.39 SC1095 transonic airfoil with the selected modifications applied ...

Figure 4.40 Aerodynamic loads SC1095 airfoil with $\mathrm{G}^{3} \mathrm{U} 2$ modification. $M=$ $0.537 \pm 0.205, f=4.25 \mathrm{~Hz}, \operatorname{Re}=6.1 \times 10^{6}$ and $\alpha=10^{\circ} \pm 10^{\circ}$ 
Figure 4.41 Pressure coefficient, Mach number contours superimposed with maximum instantaneous vorticity lines (white dashed boundaries) for the SC1095 airfoil and SC1095 with $\mathrm{G}^{3} \mathrm{U} 2 . M=0.537 \pm 0.205, f$ $=4.25 \mathrm{~Hz}, \operatorname{Re}=6.1 \times 10^{6}$ and $\alpha=10^{\circ} \pm 10^{\circ}$

Figure 4.41 (Continued) Pressure coefficient, Mach number contours superimposed with maximum instantaneous vorticity lines (white dashed boundaries) for the SC1095 airfoil and SC1095 with $\mathrm{G}^{3} \mathrm{U} 2$. $M=0.537 \pm 0.205, f=4.25 \mathrm{~Hz}, \operatorname{Re}=6.1 \times 10^{6}$ and $\alpha=10^{\circ} \pm 10^{\circ}$

Figure 4.41 (Continued) Pressure coefficient, Mach number contours superimposed with maximum instantaneous vorticity lines (white dashed boundaries) for the SC1095 airfoil and SC1095 with $\mathrm{G}^{3} \mathrm{U} 2$. $M=0.537 \pm 0.205, f=4.25 \mathrm{~Hz}, \operatorname{Re}=6.1 \times 10^{6}$ and $\alpha=10^{\circ} \pm 10^{\circ} \ldots \ldots$

Figure 4.41 (Continued) Pressure coefficient, Mach number contours superimposed with maximum instantaneous vorticity lines (white dashed boundaries) for the SC1095 airfoil and SC1095 with $\mathrm{G}^{3} \mathrm{U} 2$. $M=0.537 \pm 0.205, f=4.25 \mathrm{~Hz}, \operatorname{Re}=6.1 \times 10^{6}$ and $\alpha=10^{\circ} \pm 10^{\circ} \ldots \ldots$

Figure 4.42 Aerodynamic loads SC1095 airfoil with $\mathrm{G}^{2} \mathrm{~L} 1$ modification. $M=$ $0.537 \pm 0.205, f=4.25 \mathrm{~Hz}, \operatorname{Re}=6.1 \times 10^{6}$ and $\alpha=10^{\circ} \pm 10^{\circ}$

Figure 4.43 Pressure coefficient, Mach number contours superimposed with maximum instantaneous vorticity lines (white dashed boundaries) for the SC1095 airfoil and SC1095 with $\mathrm{G}^{2} \mathrm{~L} 1 . M=0.537 \pm 0.205, f$ $=4.25 \mathrm{~Hz}, \operatorname{Re}=6.1 \times 10^{6}$ and $\alpha=10^{\circ} \pm 10^{\circ}$

Figure 4.43 (Continued) Pressure coefficient, Mach number contours superimposed with maximum instantaneous vorticity lines (white dashed boundaries) for the SC1095 airfoil and SC1095 with $\mathrm{G}^{2} \mathrm{~L} 1$. $M=0.537 \pm 0.205, f=4.25 \mathrm{~Hz}, \operatorname{Re}=6.1 \times 10^{6}$ and $\alpha=10^{\circ} \pm 10^{\circ} \ldots \ldots$

Figure 4.43 (Continued) Pressure coefficient, Mach number contours superimposed with maximum instantaneous vorticity lines (white dashed boundaries) for the SC1095 airfoil and SC1095 with $\mathrm{G}^{2} \mathrm{~L} 1$. $M=0.537 \pm 0.205, f=4.25 \mathrm{~Hz}, \operatorname{Re}=6.1 \times 10^{6}$ and $\alpha=10^{\circ} \pm 10^{\circ} \ldots \ldots$ 
Figure 4.43 (Continued) Pressure coefficient, Mach number contours superimposed with maximum instantaneous vorticity lines (white dashed boundaries) for the SC1095 airfoil and SC1095 with $\mathrm{G}^{2} \mathrm{~L} 1$. $M=0.537 \pm 0.205, f=4.25 \mathrm{~Hz}, \operatorname{Re}=6.1 \times 10^{6}$ and $\alpha=10^{\circ} \pm 10^{\circ} \ldots \ldots$ 


\section{Nomenclature}

\begin{tabular}{|c|c|}
\hline Symbol & Definition [units] \\
\hline$a$ & Speed of sound $[\mathrm{m} / \mathrm{s}]$ \\
\hline$c$ & Airfoil chord length $[\mathrm{m}]$ \\
\hline$C_{d}$ & Section drag coefficient [-] \\
\hline$C_{f}$ & Section friction coefficient [-] \\
\hline $\mathrm{Cl}$ & Section lift coefficient [-] \\
\hline$C_{l} / C_{d}$ & Section lift-to-drag ratio [-] \\
\hline$C_{m}$ & Section pitching moment coefficient [-] \\
\hline$C_{p}$ & Pressure coefficient $[-]$ \\
\hline$I$ & Turbulence intensity [-] \\
\hline$k$ & Reduced frequency [-] \\
\hline$M$ & Mach number [-] \\
\hline$P_{\infty}$ & Air pressure $[\mathrm{Pa}]$ \\
\hline$r / R$ & Rotor radial location $[-]$ \\
\hline$R$ & Rotor radius $[\mathrm{m}]$ \\
\hline $\operatorname{Re}$ & Reynolds number [-] \\
\hline$t$ & time $[\mathrm{s}]$ (Chapter 3) and Airfoil thickness [m] (Chapter 4) \\
\hline$U$ & Freestream velocity $[\mathrm{m} / \mathrm{s}]$ \\
\hline$y^{+}$ & Dimensionless wall distance [-] \\
\hline$\alpha$ & Angle of attack [deg.] \\
\hline$\mu$ & Advance ratio $[-]$ \\
\hline
\end{tabular}




$\begin{array}{ll}v_{\infty} & \text { Air kinematic viscosity }\left[\mathrm{m}^{2} / \mathrm{s}\right] \\ \rho_{\infty} & \text { Air density }[\mathrm{kg} / \mathrm{m} 3] \\ \psi & \text { Blade azimuth [deg. }] \\ \Omega & \text { Angular velocity or Rotational frequency }[\mathrm{rad} / \mathrm{s}]\end{array}$

\section{Acronyms}

2D

AoA

BFS

CAMRAD

CFD

CFS

DBCS

FFS

NASA

RANS

URANS

SST

SA

STVG

\section{Definition}

Two Dimensional

Angle of Attack

Backward Facing Step

Comprehensive Analytical Model of Rotorcraft

Aerodynamics and Dynamics

Computational Fluid Dynamics

Constant Freestream

Density-based coupled solver

Fluctuating Freestream

National Aeronautics and Space Administration

Reynolds Averaged Navier-Stokes

Unsteady Reynolds Averaged Navier-Stokes

Shear Stress Transport

Spalart-Allmaras

Surface-based Trapped Vortex Generator 


\section{Chapter 1}

\section{Introduction}

\subsection{Motivation}

The main purpose of lifting surfaces for fixed-wing or rotary-wing aircraft is to produce lift as efficiently as possible i.e. without significant penalty in drag or pitching moment. In addition, the lifting surfaces shall be able to function at the designated angles of attack without stall. Although pitching moment can essentially be viewed as a side effect of generating lift, it is an important factor that affects the flight performance of both fixed-wing aircraft and rotary-wing aircraft [1]. For fixed-wing aircraft, the pitching moment calls for a method to counter this moment (for example via a horizontal stabilizer for conventional configuration) while for rotary-wing aircraft it is the source of potentially excessive vibration in the pitch links, limiting the forward flight performance of a helicopter.

There are several ways of increasing lift of a wing, which one can classify into active and passive flow control methods.

Active flow control methods mean achieving close to optimum performance in multiple flight regimes, usually via altering the geometry of the lifting surface or the state of the boundary layer via some type of mechanism. For fixed-wing aircraft, examples include high-lift devices (leading edge slats and/or trailing edge flaps) and boundary layer suction/blowing or the active control of airfoil shape (for more details, see Chapter 2). While some of these methods are well established for certain applications, they are 
typically used during takeoff and landing only, with limited or no capability to control the flow in other flight regimes such as transonic cruise for fixed-wing aircraft or high-speed forward flight for rotary-wing aircraft. For such applications, passive flow control technique could be more attractive.

Passive flow control - also known as flow control or flow manipulation - is very popular among researchers. Publications in this field vary from simple geometric modifications, such as vortex generators [2], to strategic control of surface roughness [3]. From these, vortex generators are one of the most popular and simplest methods to implement. Their purpose is to eliminate or delay flow separation by placing a vortex generator near the critical point at the airfoil.

In spite of the intensive research in active flow control methods, no viable methods have been so far found for two very important flight regimes affecting the fuel efficiency and the performance of the aircraft: transonic cruise for commercial passenger jets and forward flight for helicopters.

During transonic cruise of a fixed-wing aircraft, the flow velocity over the upper surface of the wing will increase to such extent that eventually regions of supersonic flow will appear, which, when decelerated back to the subsonic freestream flow, will generate a shockwave on the upper surface of the wing (Fig. 1.1). The presence of the shockwave leads to flow separation and an associated increase in drag and as a result in fuel consumption too. 


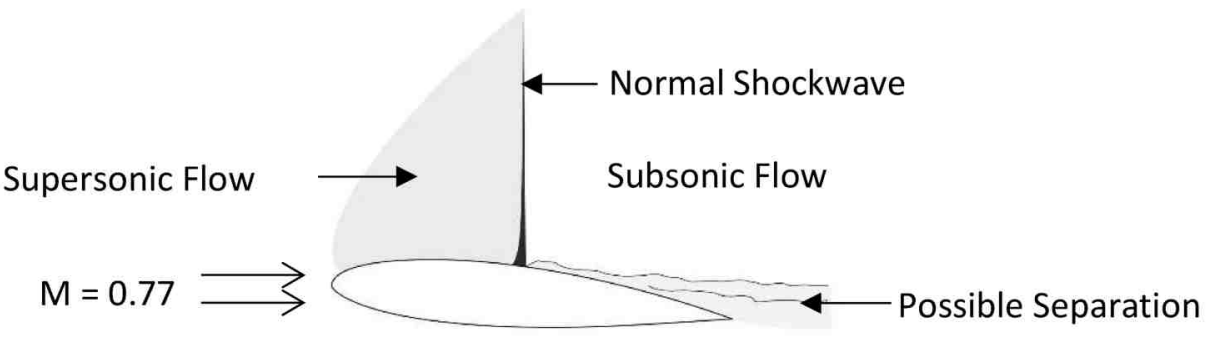

Figure 1.1 Development of supersonic flow over an airfoil.

Passive flow control in this case could for example aim to affect the strength of the shockwave or to provide flow reattachment after the separation while generating comparable lift than the original airfoil. Moreover, if the intended passive flow control method would provide lower pitching moment than the original wing airfoil, then it could call for less counter lift from the aircraft tail and therefore to decrease trim drag as shown in Fig. 1.2.

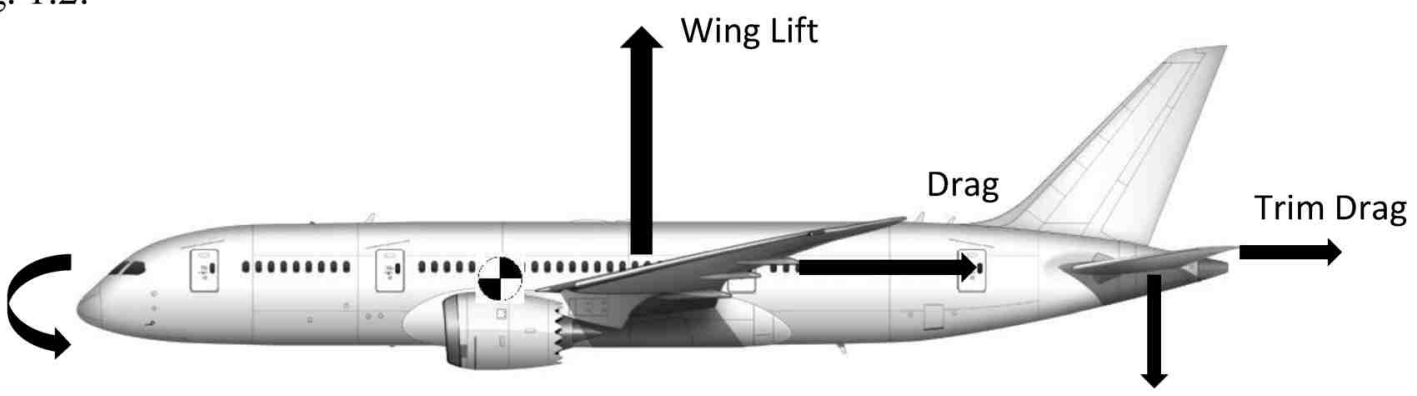

Pitching moment (-)

Tail Lift

Figure 1.2 Lift, drag and pitching moment on a fixed-wing aircraft during cruise.

For the forward flight phase of a rotary-wing aircraft, understanding of the difference between the aerodynamics of helicopter rotors in hover and forward flight is very important. Starting from hover (Fig. 1.3a), the velocity variation along the blade is azimuthally axisymmetric and radially linear with zero velocity at the root (hub) and 
maximum velocity, $V_{\text {tip }}$, at the bade tip. On the other hand, in forward flight (Fig. 1.3b), the velocity at the blade tip will be, $V_{t i p}+V$ on the right side of the rotor disk (advancing side) and $V_{t i p}-V$ on the left side of the rotor disk (retreating side), where $V$, is the forward flight speed, or freestream velocity. While the distribution of the velocity along the blade keeps the linear profile, it is no longer axisymmetric and varies in magnitude with respect to the blade azimuth angle [1].

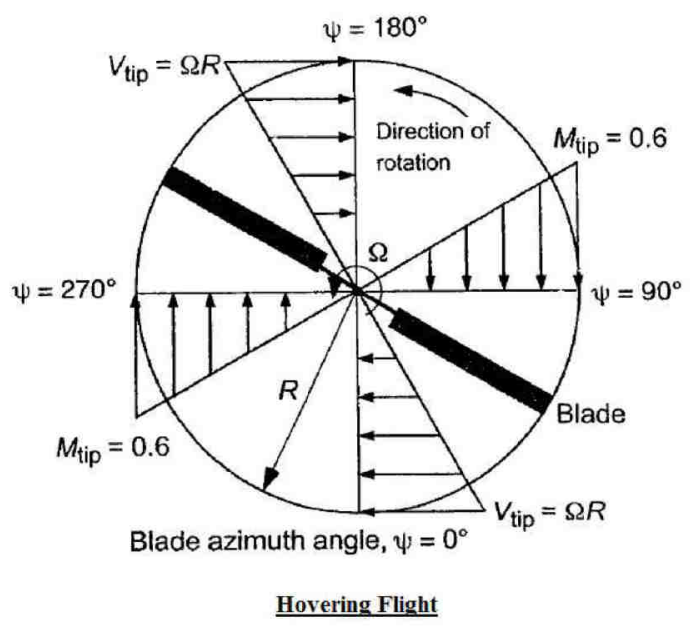

a

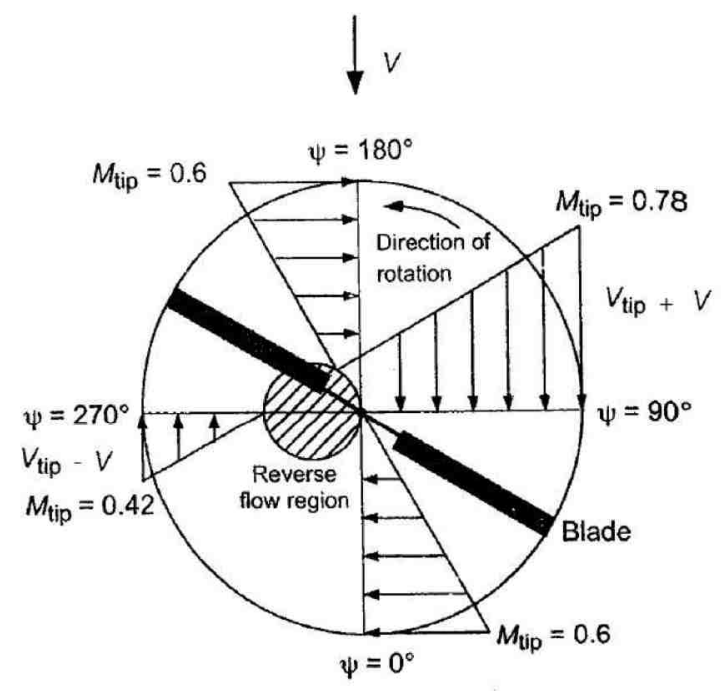

Forward Flight

b

Figure 1.3 Top view of the main rotor disk and the velocity distribution [1].

The unbalanced flow regimes and velocity distribution in the forward flight phase leads to rolling moment to the left (i.e. the retreating side of the rotor disk) if the same pitching angle would be kept at all times for all blades. To avoid this, the angle of attack (AoA) is periodically varied within each rotor revolution via the combination of blade flapping and cyclic pitch angle change via a swashplate mechanism. As a result, the blade on the advancing side of the rotor disk will experience low resultant angle of attack and possibly transonic flow and shockwaves at very high forward flight speeds. On the other hand, on the retreating side of the rotor disk the same blade will experience low speed 
flow and high angle of attack, which "side-effect" can be that it becomes subjected to the so-called dynamic stall at very high forward flight speeds as illustrated in Fig. 1.4.
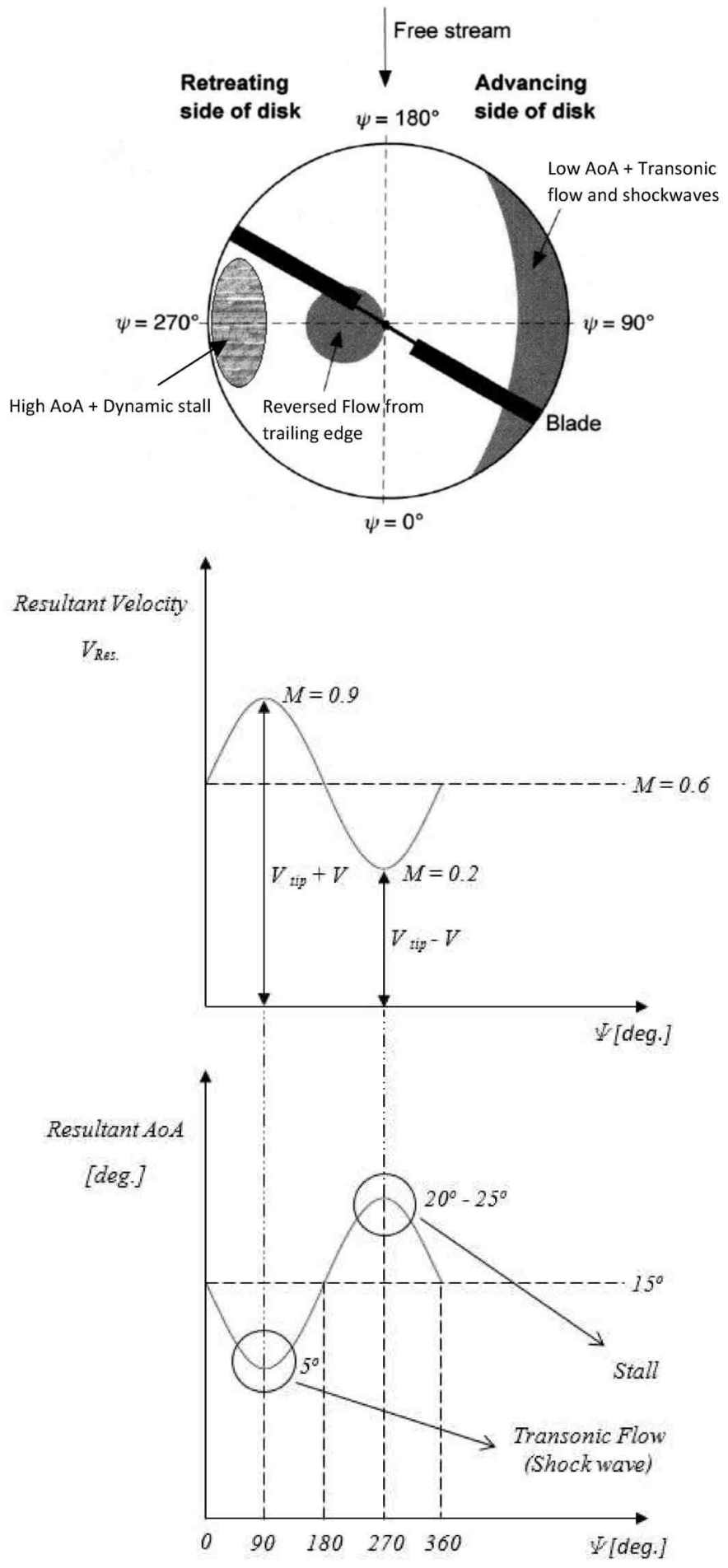

Figure 1.4 Transonic and dynamic stall regions in forward flight. 
As can be seen, the maximum forward flight speed of a helicopter is limited by the appearance of transonic flow and/or dynamic stall. These two phenomena together lead to excessive vibration, which limits the forward flight speed as well as deteriorates crew and the passengers comfort. If one could delay the onset of transonic effects and dynamic stall or to mitigate their negative effects, then the performance and ride comfort of a helicopter could be significantly improved. Achieving this with passive flow control would be highly desirable, since implementing an active flow control system in the rotating frame (of the helicopter blades) and in a high-centripetal force dominated environment is very challenging.

For rotary-wing applications, active flow control techniques typically aim to control the low-speed dynamic stall on the retreating blades in a high-speed forward flight regime, without addressing the control of transonic flow on the advancing blades. A major disadvantage of active flow control techniques is their complexity and weight when compared to passive flow control methods which offer a simpler and lighter way of flow control, although usually at a narrow range of flow conditions.

Therefore, it would be highly desired for both rotary-wing and fixed wing aircraft to find a simple yet effective passive flow control technique, which could improve the aerodynamic characteristics of an airfoil in both the transonic as well as in the low speed dynamic stall regime. This thesis aims to propose such original passive flow control concept and to examine its feasibility via Computational Fluid Dynamics (CFD). 
The proposed passive flow control technique is inspired from the idea of an airfoil with a Backward Facing Step (BFS) i.e. "stepped airfoil". Kline first discovered the advantages of such airfoil geometry accidently in 1970 for paper airplanes [14]. Because of the nature of the application - paper airplanes - stepped airfoils received only moderate attention from the scientific community initially. It was only in 1994 that Fertis have examined the feasibility of this concept for a real aircraft airfoil [20].

The main idea of the airfoil with BFS or a "stepped airfoil" is simply to replace one of the airfoil surfaces by a modified surface containing a BFS as shown in Fig. 1.5. According to Fertis [20], the BFS produces a stepwise aerodynamic discontinuity, which improves the aerodynamic characteristics and at the same time preserves most of the characteristics of the original airfoil. In addition, the turbulence resulted from the aerodynamic discontinuity will either move stall to higher angle of attacks or will produce less aggressive stall than the original airfoil. The aerodynamics of the stepped airfoil depends on the location as well as the extension of the BFS; these parameters can be easily accommodated by mechanical means to fit a certain flight condition and as a result benefiting all the improvements provided by the stepped airfoil.

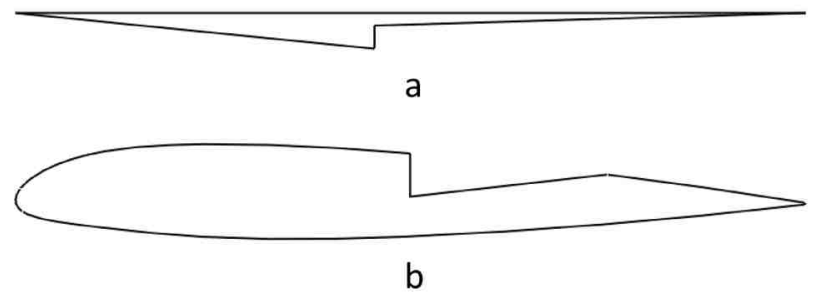

Figure 1.5 Kline- Fogleman airfoil (a) and Fertis airfoil (b). Reproduced from [14] and [20], respectively. 
In the past decades, several computational and experimental studies have been published with encouraging results $[21-25]$ but none of these examined the feasibility of stepped airfoils at high Reynolds numbers or transonic flow, i.e. for controlling the occurrence of shockwaves on airfoils.

The present research was inspired by the success gained from using BFS in one of the airfoil surfaces at low flight speeds $[21-25]$, hence, the present thesis will examine the feasibility of BFS geometries for mitigating the negative effects of transonic flow and dynamic stall. But more importantly, it aims to explore the potentials of some novel passive flow control techniques inspired by but "outperforming" BFS geometries for applications, where active flow control is difficult to apply; i.e. for fixed-wing transonic cruise or rotary-wing forward flight.

The specific goal of the study is to develop novel passive flow control concepts that could be implemented on any type of airfoil used for fixed-wing or rotary-wing aircraft, without deteriorating the original lift of the airfoil, while at the same time, mitigating the undesired characteristics (such as excessive drag or excessive pitching moment). If such geometry would exist, it could appear as a groove on the surface of the rotor blade or the wing on that portion of the span, where transonic flow or dynamic stall occurs. This promises to be a simple and more feasible alternative to any active control technology.

To the knowledge of the author, no other researchers have investigated such flow control techniques for transonic conditions and dynamic stall, and as such, this thesis presents original results. 


\subsection{Thesis Objectives}

Based on the above, the objectives of the present thesis are:

1. Develop a passive flow control concept, which can reduce transonic drag for fixedwing and rotary-wing aircraft lifting surfaces, i.e., during the cruise phase for commercial transport jets and on the advancing blades of a helicopter main rotor.

2. Develop a passive flow control concept, which can mitigate the negative effects of "classical" dynamic stall, i.e. drag and pitching moment, under oscillatory airfoil motion but constant freestream corresponding to the retreating blade flow on a helicopter rotor in forward flight. And examine whether there is an interaction between transonic flow (on the advancing blades) and dynamic stall (on the retreating blades) of a helicopter rotor. This calls for developing a methodology for simulating an oscillatory airfoil under fluctuating* freestream, where the amplitudes of pitch oscillation and freestream fluctuation are representative of helicopter forward flight conditions.

3. Develop a passive flow control concept, which can mitigate the negative effects of "real" dynamic stall, i.e. drag and pitching moment, under oscillatory airfoil motion and fluctuating freestream, where the amplitudes of pitch oscillation and freestream fluctuation are representative of helicopter forward flight conditions.

\footnotetext{
${ }^{*}$ The term used for the first time by Favier et al., 1988. See reference [64].
} 


\subsection{Research Approach}

In Aerodynamics, high Mach number and Reynolds numbers experiments, as well as unsteady Mach number experiments are considered to be as one of the most challenging ones, which are time consuming and expensive to conduct.

Since the current work proposes to examine large numbers of passive flow control concepts, for harsh environments, such as transonic flow and fluctuating freestream dynamic stall, it appeared logical to examine the feasibility of these concepts via numerical simulations first. Also, since the proposed geometries are entirely novel, it seemed logical to perform their feasibility studies on 2D airfoils / instead of 3D wings first. This allows to examine a large number of versions of the proposed flow control and to identify the most promising ones. Hence, the research objectives were met by performing Computational Fluid Dynamics (CFD) analyses of 2D airfoils under constant freestream dynamic stall, constant freestream transonic as well as fluctuating freestream dynamic stall conditions.

The concept of the research was the following: first, conduct transonic flow and dynamic stall simulations over a baseline airfoil, with careful verification and validation. Then, employ various BFS and inspired novel modifications to the baseline airfoil and compare their simulation results to the baseline airfoil. Then, conduct flow analyses of the most promising cases to understand the underlying physics behind the potential aerodynamic improvements. This approach is believed to give confidence in the simulation results and opens the path for the future practical experiments. 


\subsection{Thesis Structure}

According to the above, the rest of the thesis is structured in the following manner. In Chapter 2, a comprehensive literature review is provided on BFS based flow control methods, as well as some passive and active flow control methods, highlighting the originality of the proposed concepts. In Chapter 3, the computational method described in detail, which includes a discussion of the methods for discretization, and the method for solving the discretized system. The simulations special treatments used to provide confidence in the simulation results are also designated in Chapter 3. Chapter 4, addresses the thesis objective in sequence by analyse and discuss the results of the proposed STVG. And finally, Chapter 5 presents the conclusions and the thesis contributions along with some future work recommendations. 


\section{Chapter 2}

\section{Background and Literature Review}

\subsection{Chapter Overview}

Recall that the novel passive flow control technique proposed in this thesis was inspired by employing a Backward Facing Step (BFS) on the airfoil surface. Therefore, the literature review will start with reviewing the properties of BFS and the associated flow control methods. Following this, the airfoil with BFS on one of its surfaces will be discussed in detail by shedding light on the design aspects and the aerodynamic outcomes of each airfoil.

On the other hand, since the proposed concepts are strongly related to generating and trapping a vortex on one of the surfaces of a fixed-wing or rotary-wing airfoil, this chapter will also provide an overview of the various types of active and passive flow control of airfoils that involve a vortex as a mean of flow control, regardless of the value of Mach number. This will help to distinguish the originality of the current work later.

Eventually, a collective review about some of the important accomplishments in the field of flow control over rotorcraft blades will be presented at the end of the chapter.

Table 2.1 provides a summary list of the main publications discussed in the Literature Review, with their corresponding control method and possible application, to make it easier for the reader to navigate among them. 


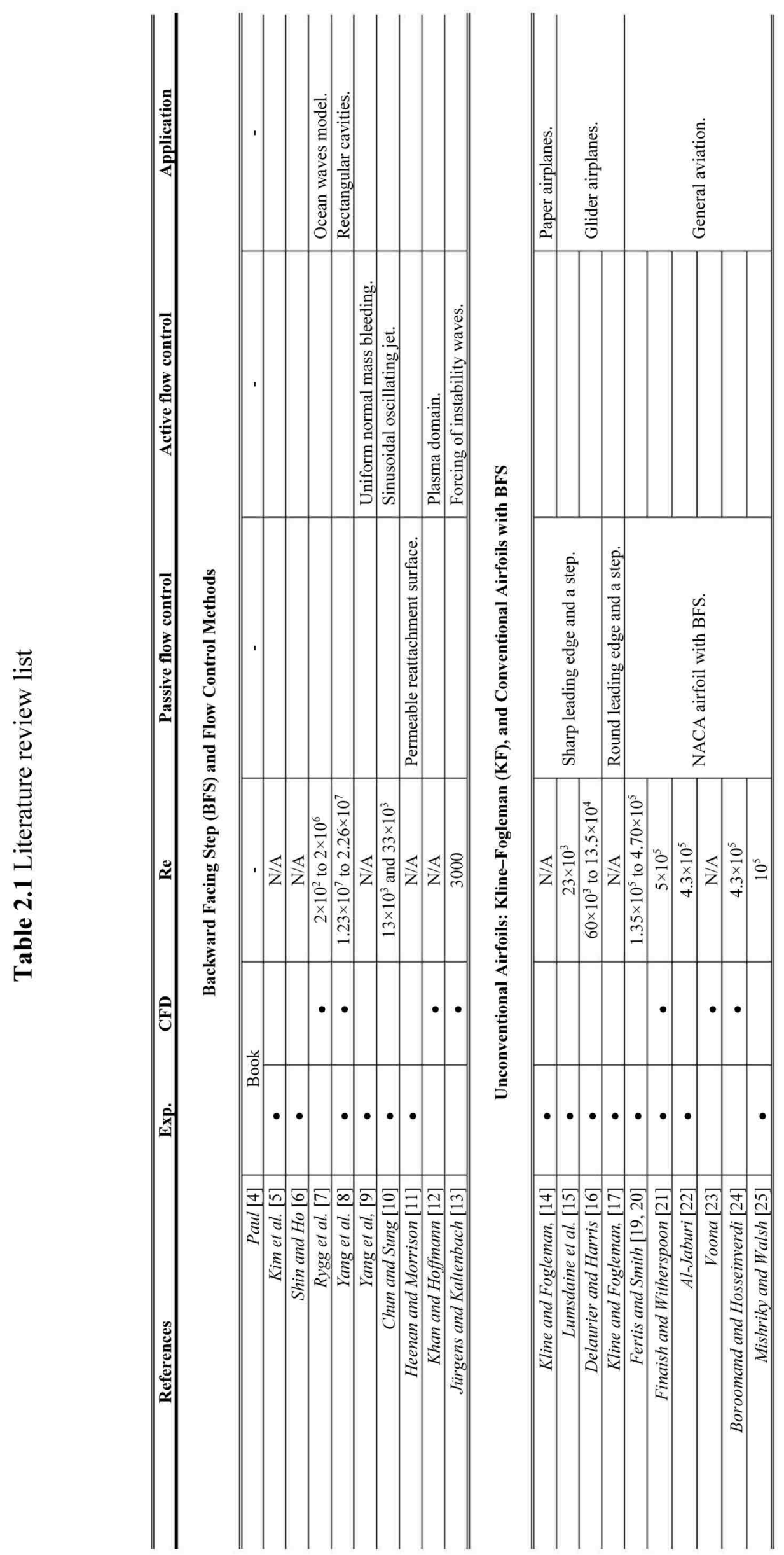




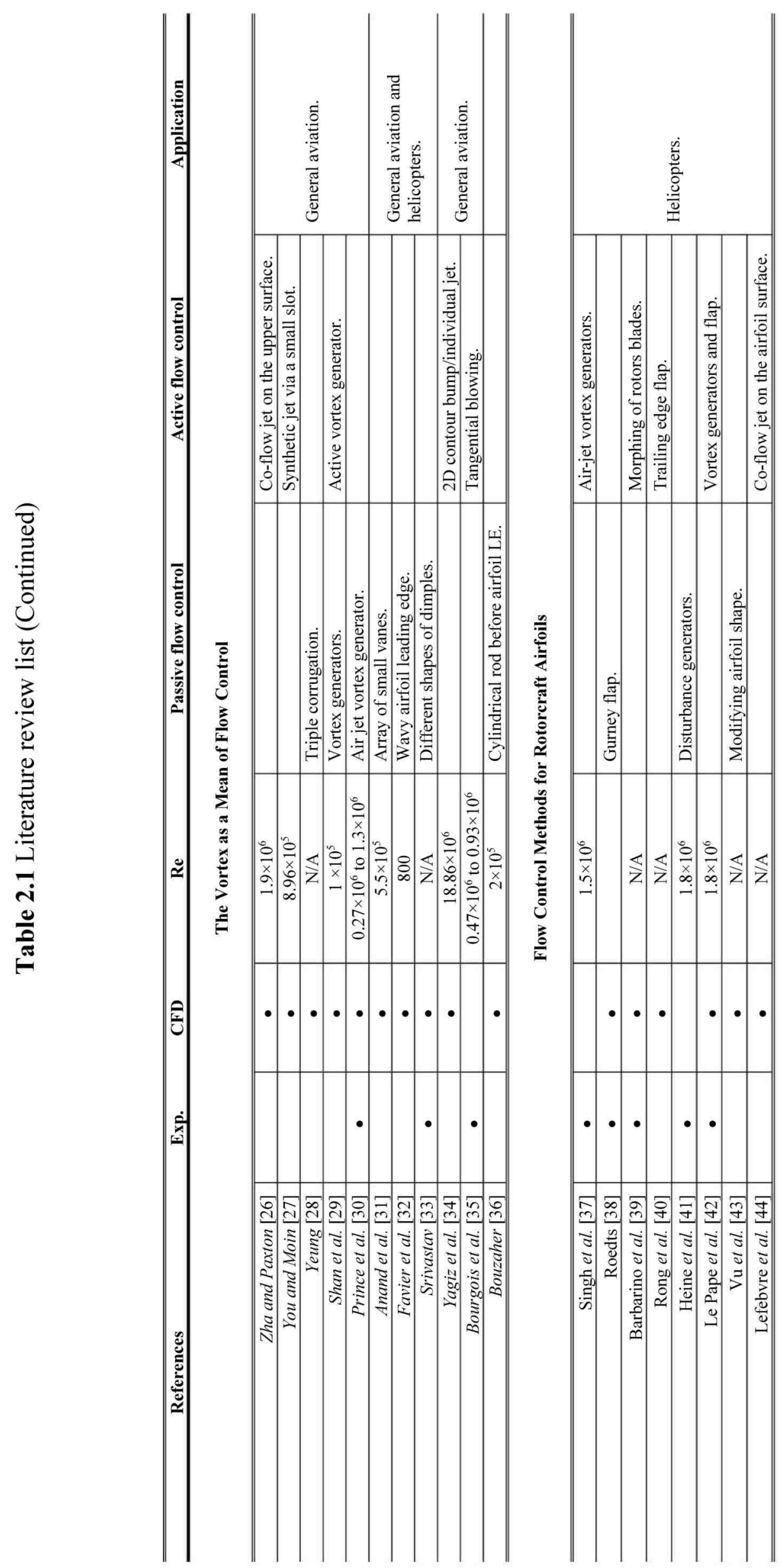




\subsection{Backward Facing Step and Flow Control Methods}

A BFS and the distribution of pressure coefficients along the step, with respect to the steps depth $(h)$ is shown in Fig. 2.1. from the experimental results of Paul [4]. In the same figure, it is evident that the effect of the step is to create a pressure rise along the surface.

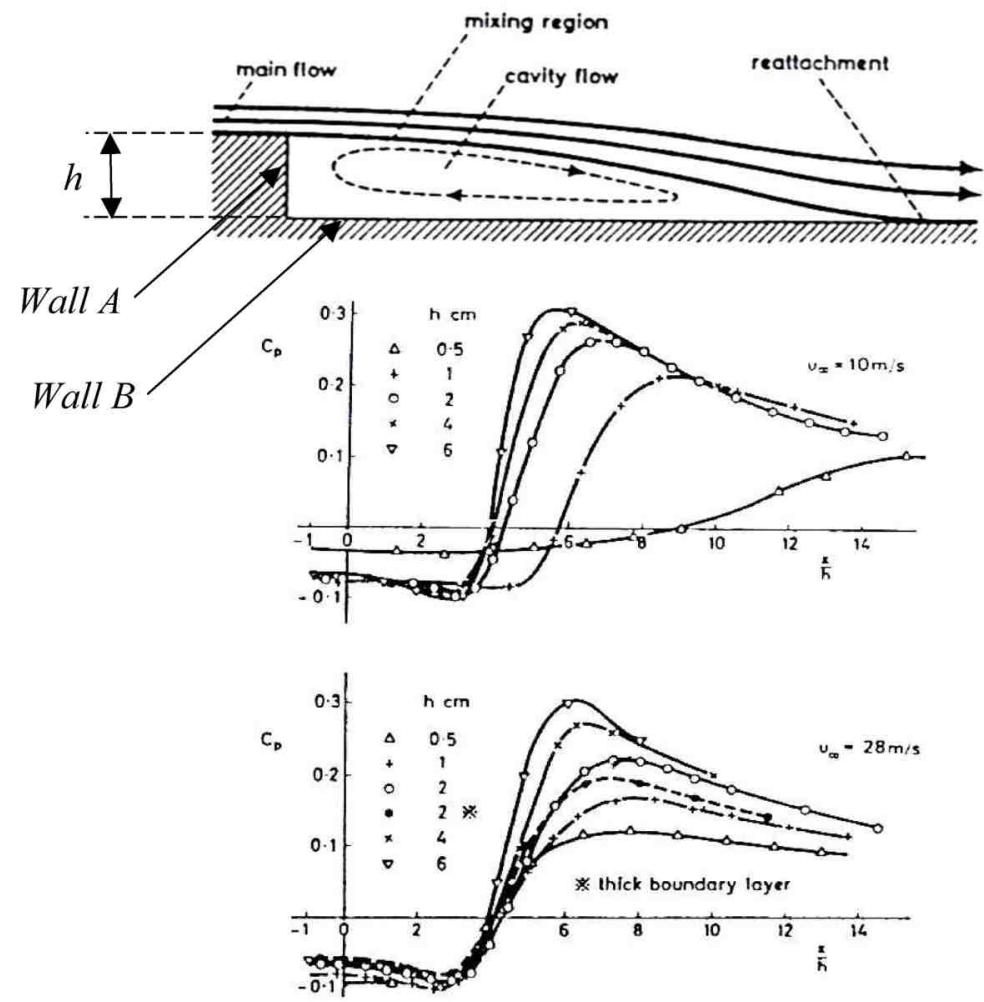

Figure 2.1 Flow over Backward Facing Step (reproduced from Paul [4]).

The data presented are averaged from different measurements. The values of $(x / h)$ that ranged from 0 to -1 represent the face of the step (Wall $A$ ), while the positive values represent the bottom of the step (Wall $B$ ). The base pressure, also known as the separation pressure, was the same regardless the thickness of the approaching boundary layer. In all cases, the base pressure was less than the free stream pressure and from Fig. 2.1, the base 
pressure decreases slightly after the step, but then rapidly increases indicating a reattachment of the separated flow inside the step.

According to Paul [4], the reason for considering the base pressure constant (regardless of the step depth or the boundary layer thickness) is that the turbulent shear stresses that preserve the cavity flow inside the step are independent of the step's depth or the thickness of the approaching boundary layer. Fig. 2.2 shows the separation region. Note that the dividing streamline lies above the inflection point. Therefore, the turbulence and the shear stress increases in the mixing region inside the step. Velocity profiles and the turbulence intensity can also be considered independent on the step depth and the thickness of the approaching boundary layer.

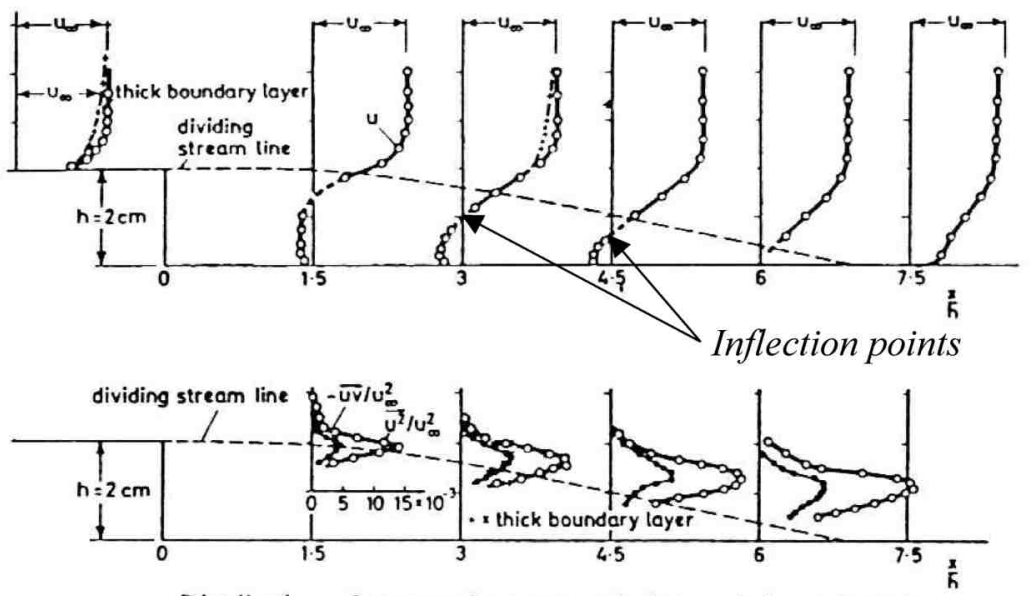

Distribution of streamwise mean velocity. turbulence intensity. and turbulent shear stress behind a backwardfacing step. $h=2 \mathrm{~cm}, u_{\infty}=$ $28 \mathrm{~m} / \mathrm{sec}$

Figure 2.2 Velocity profile distribution along Backward Facing Step (reproduced from Paul [4]).

Regarding the nature of the flow over a BFS, Kim et al. [5] conducted a practical study of incompressible flow over a BFS. They investigated the shear layer, the reattachment zone as well as the boundary layer after the re-attachment. The ratio of the 
step height to the boundary layer thickness at the separation was 2.2 and 3.3, respectively. In both cases, results indicated that the boundary layer at the separation was turbulent, turbulence intensity and shear stresses reached their maximum values in the reattachment zone, then the process was followed by a rapid diffusion near the surface of the step after the re-attachment. Once the flow was fully re-attached, it changed to a normal turbulent boundary layer before leaving the step.

Another experimental study of the re-circulation zone inside the BFS flow was conducted by Shih and Ho [6]. They studied the three-dimensional flow inside a BFS using Laser Doppler Anemometry (LDA). The step span ratio (channel width to step height) was relatively small. The three velocity components at the separation region were studied in detail. The velocity profile just before the step was two-dimensional and laminar, while inside the step the velocity profile was completely three-dimensional due to the small span ratio used.

Although the BFS is a well-known test case because of the simple geometry, it still embodies flow characteristics such as the separation point, reattachment as well as recirculating flow, which with only simple modifications can serve for other practical applications such as modeling of ocean waves [7]. Rygg et al. [7] used a rounded BFS for studying the nature of the flow of an ocean wave; the step was a $2 \mathrm{D}$ vertical version of two general circulation models, a $z$-coordinate model invented by the Massachusetts Institute of Technology, a general circulation model (MITgcm), and a $\sigma$-coordinate model originated by the Bergen Ocean Model (BOM). The flow was assumed to be homogeneous and irrotational. Reynolds numbers used in the study were based on the vertical eddy viscosity and ranged from $2 \times 10^{2}$ to $2 \times 10^{6}$. In all simulations, the separation 
occurred when the Reynolds numbers was higher or equal to $2 \times 10^{4}$. At this Reynolds number, eddies were generated at the curved surface due to the adverse pressure gradient for both models. The re-attachment point varied between $x / h=9.6$ to 10.5 . The separation point was located between $x / h=5.2$ and 5.3 and it was constant with time. Just downstream, after the separation point, there was a region with very low velocities, which is often referred to as "the dead-water region" in ocean studies. This region is larger using the MITgem than when BOM was used.

An experimental and computational investigation about the aerodynamic characteristics of transonic and supersonic flows over rectangular cavities (backward facing step joined with forward facing step) found that the shear-layer expands over the cavity leading edge and impacts on the cavity floor for closed cavity flow, whereas it overpasses in the open cavity [8]. The experiment was conducted with cavity length-todepth ratios of 6,10 and 15. Free stream angle of attack, yaw and roll was set to 0 degrees. The Mach numbers studied were 0.6, 0.8, 1.2 and 1.5 at Reynolds numbers of $1.23 \times 10^{7}, \quad 1.55 \times 10^{7}, 2.01 \times 10^{7}$ and $2.26 \times 10^{7}$ correspondingly. The static pressure distribution, on the other hand, was relatively uniform with small adverse gradients ahead of the back face inside the open cavity.

As for the BFS flow control, Yang et al. [9] conducted a practical investigation about how to reattach the separated flow over a BFS by using a uniform normal mass bleeding. The study focused on the flow directly after the step to the end of the step geometry using an LDA technique. Results showed that the mass bleeding reduced the magnitude of the reversed horizontal velocity component and the shear stress and turbulence intensity in the entire circulation region. An analysis of the vertical velocity 
component and turbulence intensity indicated that the mixing between the injected flow and the main flow velocity started at a distance of $x / h=0.4$ behind the step and increased at $x / h=0.8$ from the face of the step.

On the same track towards the separation control in BFS, one can use a sinusoidal oscillating jet injection from a thin slit near the separation line [10]. The study was experimental for Reynolds number based on the step height between $13 \times 10^{3}$ and $33 \times 10^{3}$. Major changes were obtained by using this technique, and as a result, the overall flow characteristics of separation and reattachment were significantly altered. The effect of the local forcing was observed to affect the flow at separation too, by modifying the roll-up process of the shear layer. In addition, a rapid increase in the pressure coefficient was noticed in the pressure recovery region because of the sinusoidal jet forcing. Therefore, a substantial reduction of the re-attachment lengths could be obtained by this approach.

On the other hand, Heenan and Morrison [11] came up with an interesting passive flow control technique to control the turbulent flow downstream of the BFS, by using a permeable reattachment surface. The results of this practical study showed that the global instability associated with inhomogeneous reattaching flow can effectively be removed by using a permeable reattachment surface, extending to the point where the instantaneous velocity vector near the surface is in the downstream direction. However, there is a drag penalty by using this method. It was also noticed that both of the surface pressure fluctuations and Reynolds stress have substantially reduced. The main result though is that the flow has essentially become stable.

Numerical study of unsteady supersonic flows over a BFS with plasma domain as the control technique indicated that the application of magnetic field increased the 
unsteady nature of the flow [12]. Two types of magnetic fields have been used, uniform and time-dependent. Application of the uniform magnetic field changed the flow structure in the entire domain, including an increase in oblique shock wave angle and decrease in flow velocity in the plasma domain. In the time-dependant magnetic field application, a short magnetic disturbance caused substantial disturbances in the flow field downstream of the step. Also noted was that the increase in unsteady nature of the step flow may provide some potential to enhance the mixing phenomenon for a typical shockboundary layer interaction problem.

Jürgens and Kaltenbach [13] made another numerical simulation of incompressible flow over a swept BFS. This time the study aimed to reduce the separation region by the forcing of instability waves. The Reynolds number with respect to the step height was 3,000 . To provide the instability waves, a fluid was blown and sucked periodically from a slot at the step edge. Results showed that the reattachment length could be reduced by $20 \%$ when the forcing with a sweep angle was less than 20 degrees, or by $29 \%$ for a sweep angle of 40 degrees. This change occurred because the non-linear regime of the shear layer development was reached earlier. As a result, the transition to turbulence was shifted upstream. 


\subsection{Airfoils with Backward Facing Step on the Surface}

In this section, a brief overview of the prior designs on airfoils using BFS as means of a passive flow control to improve flight characteristics.

\subsubsection{Unconventional Airfoils: Kline-Fogleman (KF) Airfoils}

In 1968, Richard Kline, a New York advertising-firm art director accidently discovered the idea of the stepped wing while making a paper airplane for his son. Later, in 1970, Kline and his colleague, Floyd Fogleman have filed for and were granted a patent for their invention [14]. Fig. 2.3 below, illustrates the KF airfoil and their paper airplane.

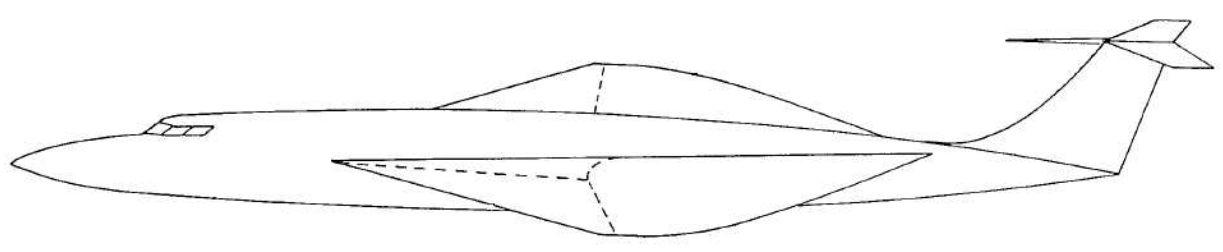

Figure 2.3 Kline-Fogleman Airfoil (reproduced from Kline et al. [14]).

Several flight tests performed on paper airplanes with the new KF airfoil demonstrated very high lift, great stability and pitching moment characteristics [14]. According to Kline, the airfoil resisted stall greatly, with a stall angle of attack pushed to as high as $30^{\circ}-40^{\circ}$, a range unreachable even by the best conventional airfoils. 
Lumsdaine et al. [15], have investigated the KF airfoil in wind tunnel in a research partially funded by NASA. The airfoil was tested with various Reynolds numbers, step longitudinal locations, step shapes and surface locations (i.e. whether it is on the upper or the lower surface). Results indicated that the KF airfoil had low lift-todrag ratio that made it not worthy for industrial use on a full-size aircraft regardless the noticeable high stability and stall resistance with significantly improved pitching moment characteristics.

In the same year, Delaurier and Harris [16] conducted another experimental study on the KF airfoil. Their results indicated that the KF airfoil could reach its maximum lift and lift to drag ratio when decreasing the step depth. It was also noticed that in contrast to classic cambered airfoils, the KF airfoil had a positive pitching moment about its aerodynamic center and that this was the main reason behind the great stability provided by such an airfoil.

Kline and Fogleman were granted another patent just a few years later for modifying their original design [17]. The new design contains a moveable part placed on the face of the step to enhance the gliding performance of the glider airplane that uses KF airfoil in its wing. Fig 2.4 is a sketch of the modified KF airfoil.

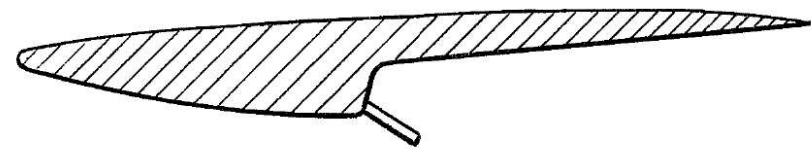

Figure 2.4 Kline-Fogleman Airfoil, $2^{\text {nd }}$ patent (reproduced from Kline et al. [17]). 
In the $21^{\text {st }}$ century, the KF airfoils have received a lot of interest from radiocontrolled (RC) model aircraft operators because of their simplicity, easy manufacturing (foam), great stability and stall resistance [18].

Powers [18] is renowned to be one of the pioneers in the world of RC airplanes and had very close communication with Kline himself who was encouraged to produce a new KF family for RC airplanes, which for this reason are called Kline-Fogleman modified airfoils (KFm) (see Fig. 2.5).

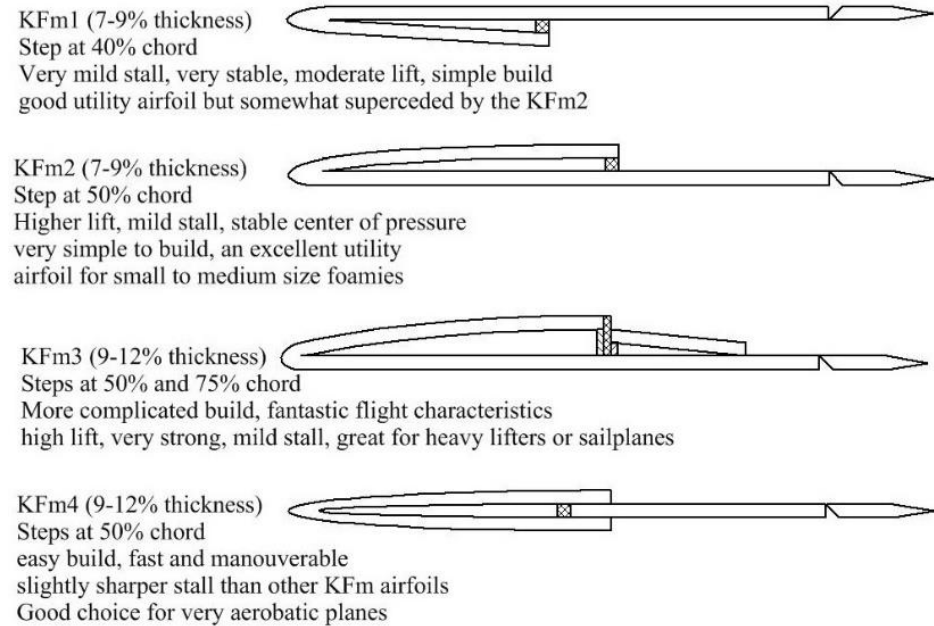

Figure 2.5 KFm family for RC airplanes (reproduced from [18]).

\subsubsection{Conventional Airfoils with Backward Facing Step}

A KF airfoil can essentially be described as a flat surface with sharp leading edge and a "step like protrusion" on the lower surface. Such airfoil was tested by Lumsdaine et al. [15] who reported that it is no better than a flat plate because of its poor lift to drag ratio.

Based on the idea of the KF airfoil, Fertis and Smith [19] have been granted a patent for a new airfoil designs with BFS on the upper surface. Fertis-Smith submitted 
two new models by essentially combining a KF airfoil with a conventional airfoil with the step at $50 \%$ of the airfoil chord on the upper surface as shown in Fig. 2.6. Wind tunnel tests showed that lift increased significantly in comparison to a conventional airfoil, while stall was either reduced or eliminated with only a slight increase in drag. Thus, in comparison to KF airfoil, a better lift to drag ratio for the full range of angle of attacks obtained [20].

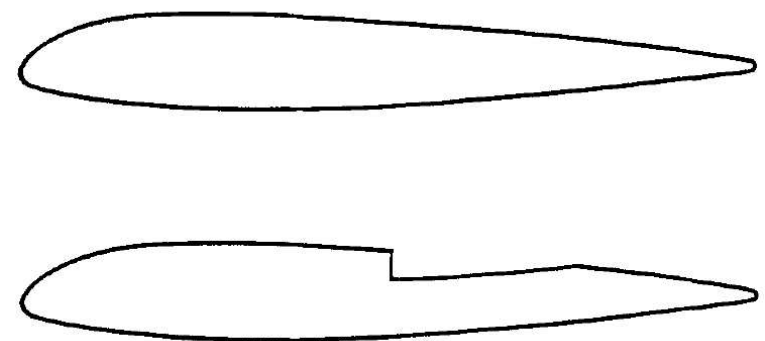

Figure 2.6 Fertis-Smith airfoils (reproduced from Fertis et al. [20]).

The airfoil used in the above study was a NACA 23012. The speed in the wind tunnel was $30.48 \mathrm{~m} / \mathrm{s}, 45.72 \mathrm{~m} / \mathrm{s}$ and $60.96 \mathrm{~m} / \mathrm{s}$. Fertis also conducted several flight tests to fully understand the capabilities of the new models. The airplane model used in the flight test was $2.134 \mathrm{~m}$ in length and the range of the flight speed was between $12.2 \mathrm{~m} / \mathrm{s}$ to $79.25 \mathrm{~m} / \mathrm{s}$. Fertis found that the flight performance increased significantly when the flight speed increased. Fig. 2.7 illustrates lift results of two different "stepped airfoil" models [20]. 


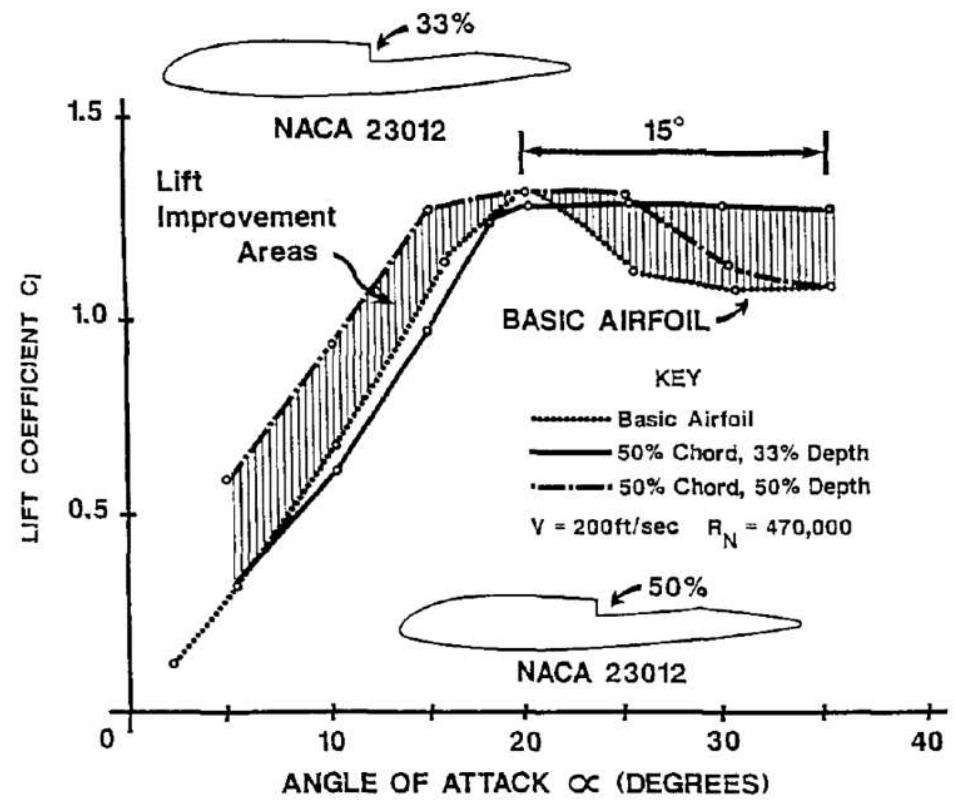

Figure 2.7 Fertis stepped airfoils with results (reproduced from Fertis [20]).

After Fertis' research, stepped airfoils gain new interest from other researchers. Finaish and Witherspoon [21] published an experimental and computational study about Fertis airfoils with a variation in the step depth. Several stepped NACA 0012 airfoils were fabricated with steps at $50 \%$ of the chord and compared to the clean airfoil. The step was installed on both the upper and the lower surfaces, respectively. The study focused on investigating the effects of flow separation and the shape of the vortex formed and how it will affect the pressure distribution at various angles of attack. The Reynolds number used in the experiments was $5 \times 10^{5}$. Results showed that the best stepped airfoil with favourable aerodynamic characteristics was the one with the step located in the lower surface at $50 \%$ of the chord and extending to the trailing edge.

Finaish's study [21] suggested to implement a flexible arrangement that allows various step configurations to be employed during flight phase because studies showed that various step configurations were good for different phases of the flight. Furthermore, 
during a single maneuver, vastly different configurations may be needed, and the best geometry needed for the intended maneuver may not be easily obvious.

With the above results, further investigation was needed to fully understand the effect of Reynolds number and flight speed on stepped airfoils and in particular, the step configurations. Therefore, Al-Jaburi [22] in his Master's thesis conducted an intensive experimental study in a wind tunnel with over 14 different stepped airfoils. The step was configured on a NACA0015 airfoil with various step depths, with all steps variants placed in the middle of the chord on both the upper surface and the lower surface of the airfoil. The Reynolds numbers based on the airfoil chord was $4.3 \times 10^{5}$, while the free stream Mach number around 0.1. Results indicated that, unlike in previous researches, not only the airfoil with the step on the lower surface (placed in the middle of the chord and extended to the trailing edge) gave a favourable outcome, but all airfoils with BFS. They yielded major improvements in the aerodynamic characteristics of the airfoil with substantial increase in lift, the lift to drag ratio, the range of the effective angles of attack and with an ability to delay stall. Table 2.2 summarises the results achieved from that study. 
Table 2.2 Results summary from Al-Jaburi [22] experimental study.

\begin{tabular}{lc|cc}
\hline \multicolumn{4}{c}{ Upper Surface Step Configuration } \\
\hline \multicolumn{1}{c}{ Highest Lift to Drag Ratio occurs at ... } & Longest Range of Angles of Attack occurs at ... \\
\hline Step Location (\%Chord) & 50 & Step Location (\%Chord) & 50 \\
Step Depth (\%Thickness) & 50 & Step Depth (\%Thickness) & 50 \\
Step Extension (\%Chord) & 25 & Step Extension (\%Chord) & 50 \\
\hline \hline \multicolumn{2}{c}{ Lower Surface } & Step Configuration \\
\hline Highest Lift to Drag Ratio occurs at ... & Longest Range of Angles of Attack occurs at ... \\
\hline Step Location (\%Chord) & 50 & Step Location (\%Chord) & 50 \\
Step Depth (\%Thickness) & 20 & Step Depth (\%Thickness) & 50 \\
Step Extension (\%Chord) & 25 & Step Extension (\%Chord) & 50 \\
\hline \hline
\end{tabular}

Several investigations were conducted later, as an example, Voona [23], Boroomand and Hosseinverdi [24] and lately Mishriky and Walsh [25]. They have all reported that the aerodynamic outcomes from stepped airfoils are strongly dependent on the baseline airfoil, i.e., it differs from one baseline airfoil to another, a fact that supports the findings of [21] and [22] above.

\subsection{The Vortex as a Mean of Airfoil Flow Control}

In this section of the literature review, some of the airfoil flow control technique attempts (both passive and active) that involves the vortex in their flow control concepts will be briefly discussed.

A circulation-based flow augmentation control method using co-flow jet on the upper surface of the airfoil proposed by Zha and Paxton [26], as shown in Fig. 2.8. The concept suggested placing an injection slot behind the leading edge and a suction slot just before the trailing edge. The baseline airfoil used in the study was a NACA2415. 
Numerical subsonic flow simulations have been conducted. Results showed that for low angles of attacks, the co-flow jet increased the lift while dramatically reducing drag. However, when the angle of attack increased, both the lift and drag increased well beyond the values with no co-flow control.
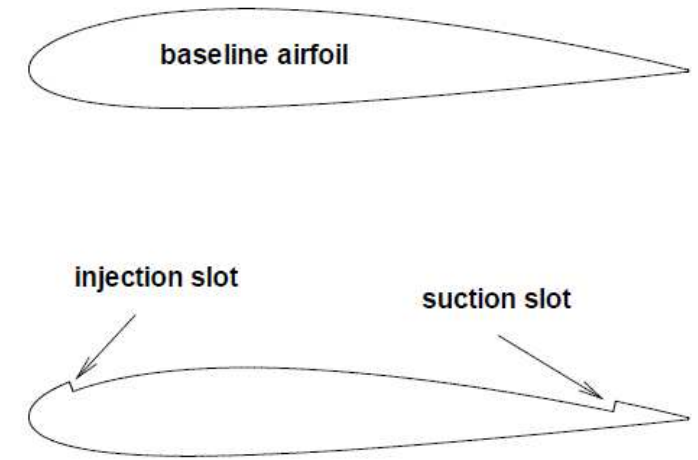

Figure 2.8 Baseline airfoil NACA 2415 and the airfoil with co-flow jet slot (reproduced from Zha and Paxton [26]).

On the same flow control track, You and Moin [27] performed a Large Eddy Simulation (LES) study on an airfoil with synthetic jet control via a small slot connected to a cavity inside the airfoil that produced oscillatory synthetic jet (Fig. 2.9). The slot was located just behind the leading edge of the airfoil. The study aimed to understand the effects of flow control on the boundary layer properties, flow separation, and lift enhancement. The airfoil used in the study was a NACA0015 at a Reynolds number of $8.96 \times 10^{5}$. Results proved that the synthetic jet actuation was able to effectively delay the start of flow separation and to significantly increase the lift coefficient compared to the uncontrolled airfoil. 


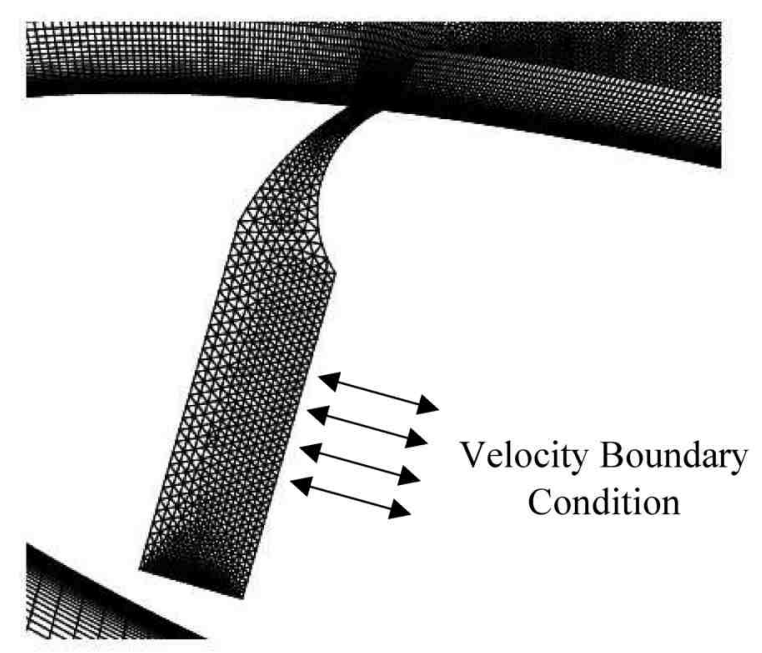

Figure 2.9 Computational mesh of the synthetic jet inside the airfoil body (reproduced from You and Moin [27]).

In another computational study, Yeung [28], triple corrugation on the upper surface of the airfoil was used as a passive flow control technique. The corrugations started just after the airfoil leading edge in a consecutive manner. Joukowsky's airfoil was used as the base airfoil for comparison. Results indicated a $10 \%$ enhancement in the lift force when using the corrugation configuration because of the vortices generated inside those cavities.

In a numerical study by Shan et al. [29], passive and active flow control with vortex generators over a NACA 0012 airfoil were examined at an angle of attack of 6 degrees. It was found that the passive vortex generators could partially eliminate the flow separation by reattaching the separated shear layer to the airfoil over a significant range. Meanwhile, with the active vortex generator, flow control was more effective, and the separation zone was not visible in the time-averaged results.

Prince et al. [30] came up with a passive air jet vortex generator-based flow control system, see Fig. 2.10. The proposed system was studied both computationally, 
experimentally and aimed to delay or suppress the trailing edge boundary layer separation and associated stall. The system consisted of an array of small orifices distributed span wise, which were embedded in the surface near the wing leading edge and supplied by a pressurized air reservoir inside the wing. Longitudinal vortices have been induced by the interaction between the jets emitting from each orifice and a free stream fluid flow.
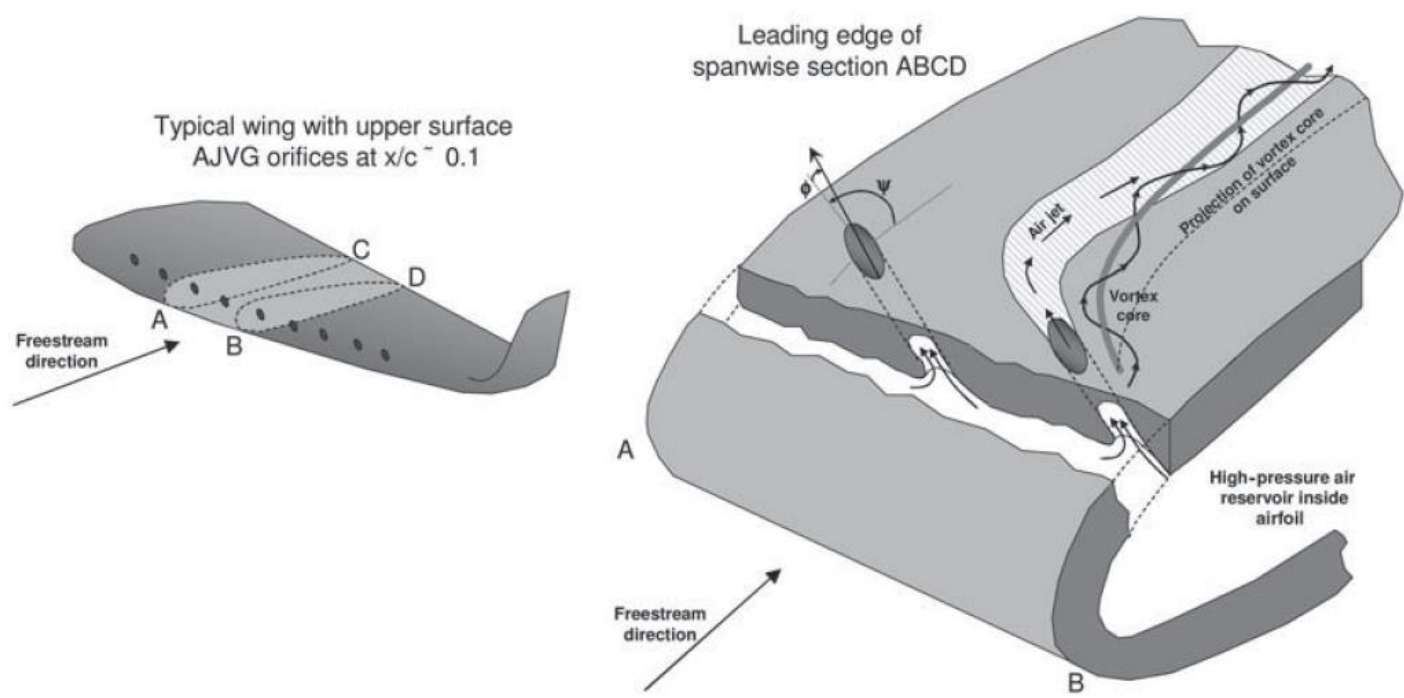

Figure 2.10 Geometry of a leading-edge array of air-jet vortex generators with illustration of the physics of longitudinal vortex formation (reproduced from Prince et al. [30])

The wings sections used were NACA 23012C and NACA 632-217. For NACA 23012C, experiments were performed with Reynolds number of $0.7 \times 10^{6}<\operatorname{Re}<1.1 \times 10^{6}$, while for NACA $63_{2}-217$, the experiments were performed with $0.27 \times 10^{6}<\operatorname{Re}<1.3 \times 10^{6}$. Results showed that the system was able to effectively delay trailing edge flow separation and stall to higher angles of attack. 
On the other hand, using an array of small vanes as vortex generators proved to be also useful [31]. The vanes could be placed in the suction side of the wing (upper surface) and were be able to delay stall and to increase lift with a recognizable reduction in drag at higher angles of attack. This study was purely computational, and the airfoil used in the study was NACA 0012 with a Reynolds number of $5.5 \times 10^{5}$.

A wavy airfoil leading edge, inspired by humpback whale flippers was proposed by Favier et al. [32]. The study was numerical only and conducted for low Reynolds numbers simulating finite wing. The wavy leading edge designed used a span wise sinusoidal function, in which its amplitude and wavelength constituted the parameters of passive flow control. A NACA 0020 wing placed at 20 degrees angle of attack with a massive leading-edge separation was used for the comparison. Results showed that when the wavelength was approximately equal to the chord and the amplitude was $7 \%$ of the chord, the shedding regime disappeared, and the flow was dominated by the streamwise structures generated by the bumps, while and the boundary layer was partially attached to the wall in correspondence with the crests positions.

Another flow control concept with multiple different shapes of dimples (outward and inward) was presented by Srivastav [33]. Subsonic flow over a NACA 0018 airfoil was studied experimentally and numerically and the results were compared. It was found that the use of the dimples delayed the boundary layer separation by creating more turbulence over the airfoil surface and as a result, reduce wake formation. In addition, the lift coefficient increased with some penalty in the drag coefficient.

According to Yagiz et al. [34], wave drag in a transonic flow may be reduced by using two-dimensional contour bump or an individual jet actuator, or the hybrid control 
that includes both these control devices. The airfoil used in the study was RAE 5243, a naturally laminar airfoil. The study was numerical, and once the flow solution for given parameters was obtained, the search for the optimum parameters to reduce the total drag of the airfoil was made via an optimizer.

Bourgois et al. [35] conducted an experimental study on an ONERA D profiled wing with a chord of $0.35 \mathrm{~m}$, modified with micro-jets and tangential blowing actuator to control the flow over the airfoil. They found that these devices were able to delay separation and improve aerodynamic performances of the wing. The experiments were conducted at Reynolds numbers of $0.47 \times 10^{6}-0.93 \times 10^{6}$. While the range for the angle of attack varied between -2 to 20 degrees. Flow visualizations by Particle Image Velocimetry (PIV) showed that, both actuators tend to increase the lift coefficient beyond stall and that the areas of the separated flow have been eliminated.

Flow separation control could also be achieved by placing a cylindrical rod in front of the leading edge of the airfoil [36]. In a LES study using ANSYS FLUENT 13, [36] showed that the effect of this rod is to accelerate the transition of the airfoil boundary layer by the interaction between the rods wake and the boundary layer. The airfoil used was a NACA 2415 at 15 degrees as angle of attack. The parameters that controlled the flow were the rod distance from the leading edge $(L)$ and the elevation of the rod corresponding to the leading edge $(h)$. Results showed that the laminar flow separation disappeared completely when the optimal parameter case $(h=0$ and $L=2.5 \times d$ and $3 \times d)$ used, where $(d)$ is the rod diameter. 


\subsection{Flow Control Methods for Rotorcraft Airfoils}

Since one of the main objectives of the current research is to propose a new passive flow control for rotorcraft airfoils, this section provides a literature review on some of the blade or (airfoil) flow control design aimed at improving the performance of helicopters.

In an experimental study by Singh et al. [37], the use of air-jet vortex generators placed on the airfoil upper surface have been examined to modify or perhaps to control dynamic stall. It was found that this method affected the pitching moment of the airfoil, which exhibited a delay in the stall as well as there was a noticeable reduction in the normal force hysteresis too. The airfoil examined was an RAE9645 section; the Reynolds number and Mach number were $1.5 \times 10^{6}$ and 0.13 , respectively, and the air-jet vortex generators consisted of two spanwise arrays located at $0.12 c$ and $0.62 c$.

Roedts [38] published a computational and experimental study of a helicopter blade design with passive flow control that aimed to enhance helicopter performance. For the experimental part, maximum test section velocity was approximately $67 \mathrm{~m} / \mathrm{s}$ and the airfoil used in the study was S903. The design suggested adding a Miniature TrailingEdge Effector or a so-called "Gurney flap" at $85 \%$ of the blade chord on the lower surface. The main rotor performance was compared to that of an RAH-66 Comanche. The results showed a $20 \%$ increase in the maximum velocity and $8 \%$ increase in the service ceiling on a Comanche military helicopter. Fig. 2.11 below illustrate the concept of Gurney flap. 


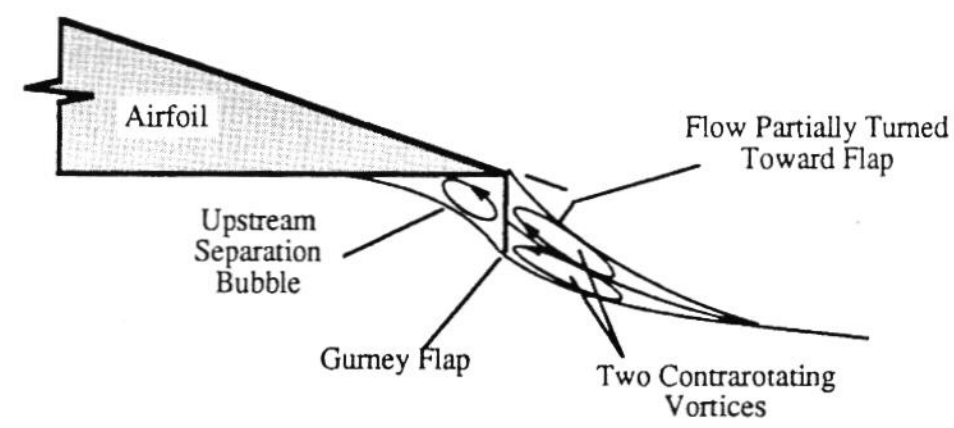

Figure 2.11 Gurney flap (reproduced from Roedts [38])

Morphing of helicopter rotor blades via chord extension was proved to be highly beneficial for stall delay, due to the ability to reduce the power near the envelope boundaries as well as to increase maximum gross weight, altitude and speed capability of the helicopter [39]. In this work, a design of a morphing mechanism extending the full chord of the airfoil (blade) was proposed. The feasibility of the design was studied using finite element analysis. The morphing cellular structure ensured that the maximum allowable local strains of the cellular structure are not exceeded even as the section undergoes a large global strain. In addition, various methods of flexible skin attachment to the morphing substructure and their ramifications were considered. After the fabrication, the design tested and proved to be functional for the purpose that was designed to serve.

Rong and PinQi [40] presented another computational study aiming to delay the dynamic stall of the helicopter retreating blade. The analysis included a trailing edge flap placed at $0.4 R-0.8 R$ from the root of the main rotor disk. The airfoil and the flap were NACA 64A006, and the ratio of the flap and the airfoil chords was 0.2. The Mach number used in the simulation was 0.5 . The flap was designed to deflect according to a 
sinusoidal signal with the movement starting from the equilibrium position at zero-degree azimuth of the rotor disk. Numerical results indicated that the flap could delay the dynamic stall of the retreating blade.

On the other hand, it was found that by using disturbance generators placed in the stagnation region of the airfoil could help to reduce the negative pitching moment peak and hysteresis effects, hence, to control the dynamic stall of the blade [41]. Three different disturbance generators were used; circular cylinders, forward equilateral wedges and backward equilateral wedges. The study was experimental, and the airfoil used was OA 209 subjected to high-speed Particle Image Velocimetry (PIV) and simultaneous pressure measurement. The experimental Reynolds and Mach numbers were $1.8 \times 10^{6}$ and 0.16 , respectively.

Le Pape of ONERA [42] studied experimentally and computationally active flow control of rotor blades. The study was divided into two parts; the first part aimed to examine the control of dynamic stall on the retreating blades while the second part was about improving the aerodynamics of the blade. In both parts, two different active flow control methods were used. Regarding the first part, a blade (airfoil) design with actively deployable vortex generators was used, with the actuator arrays placed at the leading edge of the airfoil. The purpose of the design was to delay the dynamic stall of the blade. As for the second part, an actively controlled flap aimed to enhance the aerodynamic performance of the blade. The flap was located at the trailing edge of the airfoil for a certain spanwise extension. Both systems actively actuated at various frequencies during the rotation of the main rotor. Reynolds number used in the experiments was $1.8 \times 10^{6}$ and the airfoil used in the study was OA 209. The results from this investigation were 
promising and showed that the design outcome was reliable and could be developed for applications in the future.

Modifying the shape of the airfoil for hover performance improvement was the focus of a numerical study made by $\mathrm{Vu}$ et al. [43]. This research proposed new geometry representation algorithms to obtain an optimal shape for helicopter rotor blades, which would enhance the aerodynamic performance of helicopters in hover. The optimization process was accomplished by integrating several programs developed by Lee himself. The new design included varying not only the airfoil coordinates but also the twist, the taper ratio, the point of taper initiation, blade root chord, and coefficients of the airfoil distribution function. The baseline airfoil for the study was NACA 0012 and the objective function for the optimization was the power in hover. Results of the modified blade shape indicated a reduction in the required hover power by $7.4 \%$ and an increase in the Figure of Merit $\left(P_{\text {ideal }} / P_{\text {hover }}\right)$ by $6.5 \%$, which was considered as an enhancement to the rotor blade original design.

One of the famous airfoil active flow control is the co-flow jet. In this method, a significant enhancement in the aerodynamic characteristics of a pitching moment could be achieved beside a noticeable delay of dynamic stall [44]. The authors of this study used an Unsteady Reynolds Average Navier-Stokes CFD code (URANS) at Mach number 0.4 with a reduced frequency of 0.1 . Three airfoils were subjected to the study NACA 1209, NACA 2209a and NACA 2209b. The simulated flow was transonic with shockwave-boundary layer interaction, and the maximum pitch angle was 10 degrees, the pitching angles studied was 5, 7.5 and 10 degrees respectively. However, because of the 
absence of experimental data at $\mathrm{M}=0.4$, the simulation validation was done at $\mathrm{M}=0.3$ for the SC 1095 airfoil only.

\subsection{Flow Control Methods for Airfoil Dynamic Stall}

From the previous researches, dynamic stall is one of the most limiting factors of a helicopter's flight envelope and its delay and/or mitigation of its negative effects promises to improve the performance and ride comfort of a helicopter.

There have been several techniques proposed to mitigate the negative effects of dynamic stall. Rotor-based active flow control systems, such as the Active Twist Rotor [45, 46] Active Pitch Link [47, 48], Actively Controlled Flap, [49], pulse jet actuators [50] as well as structural control systems, such as active stiffness control [51] have been successfully demonstrated at various levels of maturity in the past two decades. However, due to their complexity, weight and/or reliability, these systems have not yet found their way into serial production. A Hub Mounted Vibration Suppressor system (HMVS), which utilizes the out-of-phase rotation of two eccentric masses has been successfully flight tested recently and is expected to appear on production helicopters soon [52]. Several fuselage-based active control systems have been developed too, however, these do not address the problem of vibration and noise at their root - i.e. on the blade - and are typically much heavier than rotor-based systems [53].

Thus, the search for a simple, reliable, lightweight, blade-based control system is ongoing, as illustrated for example by the recent work of Gardner et al. [54], who proposed the use of a semi-passive backflow flap for mitigating the effects of dynamic stall. 
However, dynamic stall on a helicopter rotor blade occurs at the simultaneous variation of the relative freestream as well as of the effective angle of attack. At moderate and fast advance ratios, the amplitude of the relative freestream fluctuation can be as high as Mach 0.3 , the amplitude of the angle of attack as high as 10 degrees and all these occur at the rotational frequency, i.e. at about $3 \sim 5 \mathrm{~Hz}$. Unfortunately, reproduction of a fluctuating freestream variation of Mach 0.3 amplitude and at a frequency of $3 \sim 5 \mathrm{~Hz}$ is extremely challenging in ground-based experiments. Therefore, the vast majority of dynamic stall studies have historically focused on considering only the variation of the pitch angle, while neglecting the fluctuation in the freestream [55-59].

There have been only a handful of researchers who conducted experimental or CFD studies of dynamic stall by considering fluctuating freestream as well. Gosselin et al. [60] has conducted a CFD study on dynamic stall with fluctuating freestream, which was dedicated to exploring transonic effects on dynamic stall in fast forward flight, but with validating the simulation only at the lower extreme of the freestream fluctuation as well as with a narrow scope of the influence of numerical parameters, such as the size of the computational domain. Table 2.3 provides a summarized literature survey regarding dynamic stall at fluctuating freestream. 
Table 2.3 Summary of prior work on fluctuating freestream dynamic stall.

\begin{tabular}{|c|c|c|c|c|}
\hline References & Exp. & CFD & "Mach Number Range & Angle of Attack Range [deg.] \\
\hline "Gosselin et al. [60] & & - & $0.18-0.78$ & " $15 \pm 10$ \\
\hline Pierce et al. [61] & $\bullet$ & & $0.031-0.044$ & $6,10,14 \pm 4,18 \pm 4$ \\
\hline Maresca et al. [62] & $\bullet$ & & $0.007-0.058$ & $15 \pm 10,20 \pm 17$ \\
\hline Brendel and Mueller [63] & $\bullet$ & & $0.013-0.015$ & $-5,0,7$ \\
\hline Favier et al. [64] & $\bullet$ & & $0.007-0.058$ & $6 \pm 6,12 \pm 6$ \\
\hline Ellsworth and Mueller [65] & $\bullet$ & & $3.9 \times 10^{-5}-4.4 \times 10^{-5}$ & 7 \\
\hline Favier et al. [66] & - & $\bullet$ & $0.007-0.072$ & $9 \pm 6,12 \pm 6$ \\
\hline Shi and Ming [67] & $\bullet$ & & $0.017-0.052$ & $0-30,0-60,15-45,30-60$ \\
\hline Shi and Ming [68] & $\bullet$ & & $0.012-0.040$ & $0-60$ \\
\hline Gompertz et al. [69] & $\bullet$ & & $0.38-0.48$ & $8-20$ \\
\hline Kerstens and Williams [70] & $\bullet$ & & $0.008-0.032$ & $0-20$ \\
\hline Kerho [71] & & $\bullet$ & $0.34-0.76$ & $10 \pm 10$ \\
\hline Martinat et al. [72] & & $\bullet$ & $0.011-0.018$ & $12 \pm 6$ \\
\hline Gharali and Johnson [73] & & $\bullet$ & $0.009-0.078$ & $10 \pm 15$ \\
\hline Hird et al. [77] & $\bullet$ & & $0.32-0.48$ & $8.5 \pm 13$ \\
\hline Hird et al. [78] & $\bullet$ & & $0.35-0.45$ & $8<\alpha<20$ \\
\hline Zhao et al. [79] & & $\bullet$ & $0.22-0.58$ & $8 \pm 6$ \\
\hline
\end{tabular}

Kerho [71], Gharali et al. [73] as well as Glaz et al. [74, 75] have conducted CFD simulations of dynamic stall with fluctuating freestream, but either without validating the results to experiments and/or without exploring the transonic effects that could appear and affect the flow hysteresis $[71,74,75]$. In the case of Gharali et al. [73] the conditions were representative of wind turbines and not helicopters. Fernie et al. [76] and Hird et al. $[77,78]$ conducted experimental studies, but with freestream fluctuations corresponding to very modest advance ratios. Recently, Zhao et al. [79] conducted a CFD study with Mach number ranged from $0.2-0.6$ in an effort to study the effect of such a freestream velocity fluctuation on the aerodynamic characteristics of an airfoil subjected to oscillation. However, although the Mach number range suggested the occurrence of transonic flow on the advancing blade section, its effect was absent in the hysteresis loops and flow visualizations. 


\subsection{The Novelty of Present Research}

From the above literature review, it is clear that both kinds of vortex generators (passive and active) can significantly enhance the performance of a fixed-wing aircraft or rotary-wing aircraft. However, in all previously mentioned research, the focus was on a particular aspect of the aerodynamics characteristics, i.e. the lift enhancement or the mitigation of dynamic stall only, without further investigation of the other practical aspects of the design such as effects on drag or stability of the control system during the stall phase itself.

Furthermore, all the previously investigated designs (passive or active) were designed and tested for one type of baseline airfoil, or airfoils from the same family, or have relatively similar geometries. In addition, the flow regimes were limited or rather low subsonic with moderate Reynolds numbers, often not representative of actual flight regimes. And finally, many of the designs may be considered as rather futuristic because of their design complexity.

In general, active control systems are promising, but they are difficult to implement in practice, especially in harsh environments such as the transonic flow of the commercial jets or the flow conditions corresponding to the forward flight of a rotorcraft where the main rotor dominated by strong centripetal force and shock-induced dynamic stall.

As a result, the present research aims to prove the ability of a novel passive flow control technique to reduce drag and pitching moment and at the same time to increase the lift-to-drag ratio of airfoils in subsonic and transonic flow regimes, i.e. in the 
transonic cruise phase of a commercial jet or shock-induced dynamic stall for a helicopter in forward flight.

Furthermore, the research will investigate for the first time the performance of stepped airfoils in the harsh environments mentioned above (termed as "first generation" in this work) as well as a vast variety of novel passive flow control concepts (termed as "second, third and fourth generation" in this work), designed to be implemented on any type of airfoil or wing. These novel passive flow control generations should be designed as a potential alternative to active flow control systems. 


\section{Chapter 3}

\section{Computational Setup and Procedures}

\subsection{Chapter Overview}

In this chapter, the methods and techniques used for the numerical study of the current work are described. Because the study investigates more than one case, and each one is different from the others, the chapter was sectioned by test cases.

The chapter starts by presenting an overview of the numerical method employed by the ANSYS FLUENT code. Following that, the computational procedures of $2 \mathrm{D}$ transonic flow and 2D dynamic stall (at constant and fluctuating freestreams) will be discussed, respectively. In each section, the test case, computational domain, mesh generation, verification and validation will be discussed. Furthermore, in some cases, special attention is paid to prove the robustness of the simulations setup. 


\subsection{Numerical Method}

The ANSYS FLUENT code was used for the numerical simulations. According to the Theory Guide of the code [89], the Reynolds Averaged Navier-Stokes equations (RANS) as well as the unsteady version (URANS) are solved by utilizing a finite volume spatial discretization. In this approach, FLUENT uses a cell centered control volume, in which the computational domain is discretized into a mesh as shown in Fig. 3.1 below. The code allows to employ structured, unstructured and hybrid structured-unstructured meshes. The latter one was used for all simulations presented in this work.

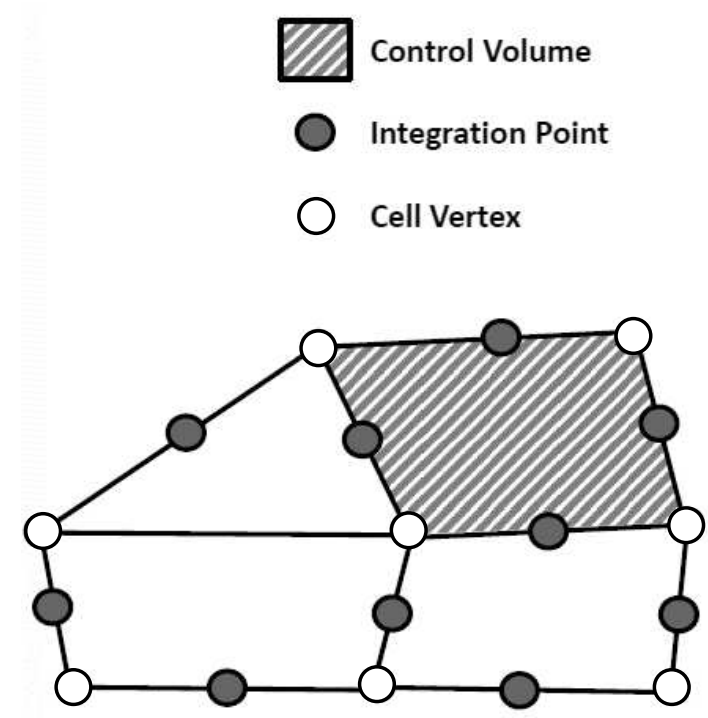

Figure 3.1 FLUENT control volume definition (reproduced from [80]).

Regarding the ANSYS FLUENT solver, it utilizes two-distinct solvers, a Pressurebased solver and a Density-based coupled solver (DBCS). In the current study the DBCS solver was used because it is more suitable for compressible flow simulations, especially, 
high Mach number flows [81]. In the DBCS Solver, equations for continuity, momentum, energy, and species, if required, are solved in vector form. Pressure is obtained through the equation of state. Additional scalar equations are solved in a segregated fashion. The density-based solver can use either an implicit or explicit solution approach. The current study utilized the implicit solver because this is generally preferred to the explicit one, which has a very strict limit on the time step size [80].

Since all the simulations in the study are compressible flow simulations, the gradient method was set to be "Green-Gauss Node Based"; this is more computationally expensive than the other methods but is more accurate. "Second Order Upwind" discretization was used for both the turbulent kinetic energy and specific dissipation rate to accurately predict drag because the default first order schemes are not sufficient [80].

The fluxes were resolved via the Roe-FDS scheme with $2^{\text {nd }}$ order upwind flow type. Sutherland's law was employed for the variation of viscosity with temperature. Depending on the flow simulated, two turbulent models were used in the study; SpalartAllmaras (SA) and SST $k-\omega$.

Regarding the 2D transonic flow, the numerical models for this kind of flow according to ANSYS FLUENT solver theory guide [81] should be set to SST $k-\omega$ model.

On the other hand, for the 2D dynamic stall cases under constant freestream, according to the recommendations in [71], [82] and [83], the SA turbulent model has been used. This was found to be effective and robust for a variety of flows including $2 \mathrm{D}$ separated airfoil flows. However, Richter et al. [84] found that while the SST $k$ - $\omega$ model achieved better qualitative predictions for both the main and secondary stall events, it exhibited deficiencies in the simulation of the reattachment during downstroke. 
Therefore, it was decided to use the one-equation SA turbulent model in the initial analyses phase of the study to reduce the computational costs, while the SST $k$ - $\omega$ two equations model was used in the final simulations to achieve better accuracy and more reliable solution.

Although for simulating constant freestream dynamic stall the SST $k$ - $\omega$ model is generally more accurate than the SA model [84], it was found by Richter et al. [85], Klein et al. [86] and Al-Jaburi et al. [87] that it generates two extra peaks in the aerodynamic characteristics $\left(C_{l}, C_{d}, C_{m}\right)$ around the peak angle of attack. These appear to occur due to the SST $k$ - $\omega$ model producing non-physical vortices. Therefore, because of the absence of a dynamic stall experiment representative of the conditions seen by a helicopter blade section in fast-forward flight (i.e. fluctuating freestream with shockwave on the tip of the advancing blade), it was decided that for all fluctuating freestream 2D dynamic stall cases the SA turbulence model will be used for all simulations, since this is expected to capture the flow physics more credibly as well as to reduce the computational cost. 


\subsection{D Transonic Flow}

\subsubsection{Test Cases}

All test cases shown in this thesis are based on experiments reported in $[88,89]$, since it is representative of transonic flow occurring both on a commercial jet aircraft's wing in cruise as well as on the advancing blade of a helicopter in forward flight. Table 3.1 below provides the details of the test cases used in the present work.

Table 3.1 Test cases for the $2 \mathrm{D}$ transonic studies.

\begin{tabular}{|c|c|c|c|c|}
\hline \multicolumn{5}{|c|}{ Test Case 1, [88] } \\
\hline Baseline Airfoil & Airfoil Chord & $\begin{array}{c}\text { Freestream Mach } \\
\text { Number }\end{array}$ & Reynolds Number* & Angle of Attack (AoA) \\
\hline NACA 0012 & $0.635 \mathrm{~m}$ & 0.7 & $9 \times 10^{6}$ & $0^{\circ}$ to $5^{\circ}$ \\
\hline \multicolumn{5}{|c|}{ Test Case 2, [89] } \\
\hline Baseline Airfoil & Airfoil Chord & $\begin{array}{c}\text { Freestream Mach } \\
\text { Number }\end{array}$ & Reynolds Number* & Angle of Attack (AoA) \\
\hline NASA SC(3)-0712(B) & $0.1524 \mathrm{~m}$ & 0.78 & $30 \times 10^{6}$ & $0^{\circ}$ to $5^{\circ}$ \\
\hline
\end{tabular}

It is worth to mention that during the experiment the accuracy of the pressure transducers was within $\pm 0.5 \%$ for Test Case 1 [88] and it was $\pm 0.25 \%$ for Test Case 2 [89].

\subsubsection{Computational Domain}

A 2D circular computational domain was used, extending $500 \mathrm{c}$ around the airfoil (Fig. 3.2). The size of the domain was chosen after recommendations found in the literature, pertaining to the study of transonic flow via 2D-CFD simulations. In Tomas et al. [90], it was recommended that for subsonic and transonic flow regimes, a modification to the free stream conditions $\left(u_{\infty}, v_{\infty}, P_{\infty}\right.$ and $\left.\rho_{\infty}\right)$ must be applied for 2D-CFD simulations of lifting bodies. This modification is named as "Point Vortex Correction" and called 
"Vortex Correction in 2D" in Blazek [91]. Since implementing the vortex correction inside FLUENT was found to be problematic, [90] also suggested an alternative method, which comprises from increasing the size of the domain to minimize the issues associated with the far-field boundary. Blazek [91] suggested that a minimum distance of 100 chords away from the airfoil would be required for turbulent subsonic flow simulations (see Fig. 3.3). More recently, Rumsey [92] suggested in a study of 2D NACA 0012 airfoil validation case to use a domain of 500 chords around the airfoil. Therefore, this latter recommendation was used to insure the accuracy of the results. The airfoil surfaces were set to no-slip wall boundary conditions with no heat flux or heat generation. The outer edges of the domain were set as pressure far-field, with the turbulence intensity set as $I=1 \%$. 


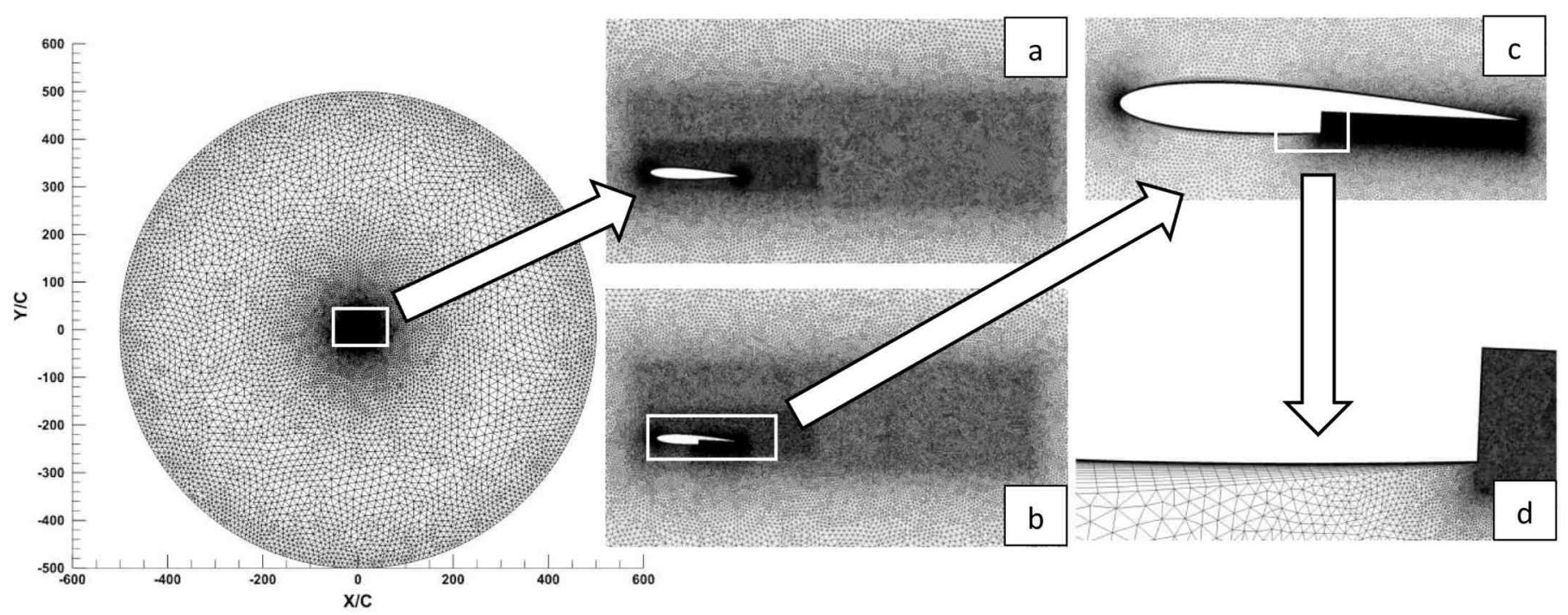

Figure 3.2 Computational domain for 2D transonic simulations: (a) refined mesh area inside the domain (baseline airfoil) (b) refined mesh area inside the domain (modified airfoil) (c) mesh density of the surface modification (d) inflation layers and cell distribution around airfoil surface.

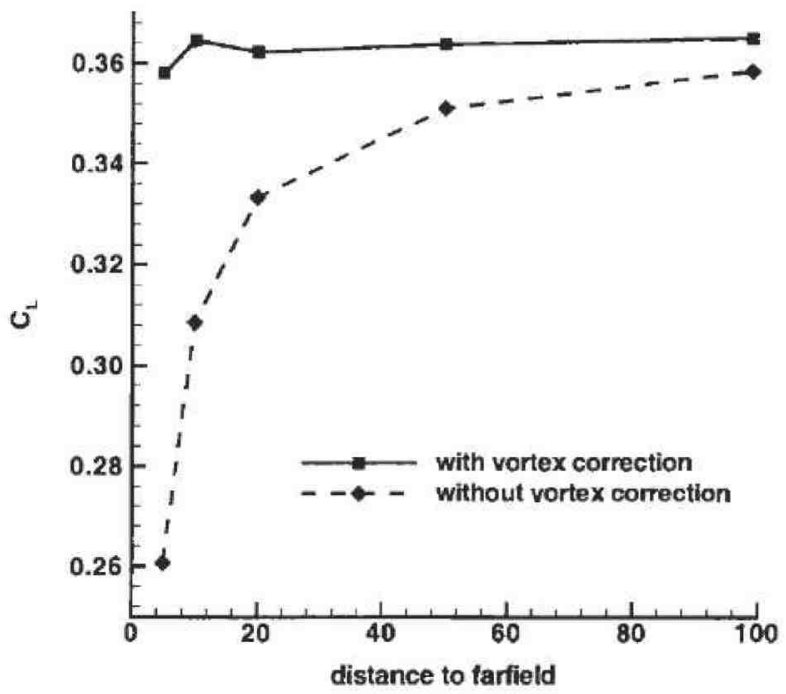

Figure 3.3 Effects of distance to the far-field boundary with and without vortex correction for a NACA 0012 airfoil at $M=0.8, A o A=1.25^{\circ}$ (reproduced from [91]). 


\subsubsection{Mesh Generation}

The total number of cells forming the computational domain was 178,738. A structured mesh consisting of rectangular cells was used only in the "inflation layer" around the airfoil, so that the boundary layer can be effectively captured. An unstructured mesh consisting of 2D triangles was used everywhere else outside of this inflation layer. The unstructured mesh was refined in the area above and downstream of the airfoil, where the shockwave induced separation is expected to appear, as well as in the area around the leading and trailing edges and also where the surface modifications on the airfoil were refined (Fig. 3.2 b and c). Exactly 650 cells were distributed along the airfoil surface. 40 layers of structured mesh were employed inside the inflation layer, with ensuring that the first spacing around the airfoil is $y^{+}<1$ for the entire simulations. Hence, no wall function was used in the turbulence model (Fig. 3.2 d).

\subsubsection{Verification}

Grid convergence analysis was performed using two airfoils, a clean airfoil without modification (baseline) and an airfoil with a modification. Both analyses were conducted at the separation angle of attack $\left(4.74^{\circ}\right)$ of the baseline airfoil to ensure that the simulations are independent of the mesh density. The coefficient of pressure $\left(C_{p}\right)$ and the $x$-component of the coefficient of friction $\left(C_{f}\right)$ were selected to be the parameters of the grid convergence analysis. The convergence criterion was set to $10^{-9}$ residual for the continuity, momentum and energy equations. 
This was accomplished by using a baseline mesh density of 178,738 cells. Also, the Courant number was varied from 5 to 200 with 3,500 iterations for each angle of attack simulation. Further refinement of the parameters above did not improve the accuracy of the results any further. Fig. 3.4 illustrates the results of the grid convergence analysis. Note that the same grid convergence test was performed for one of the modified airfoils as well (Fig. 3.4.b), which geometrical details will be explained later.
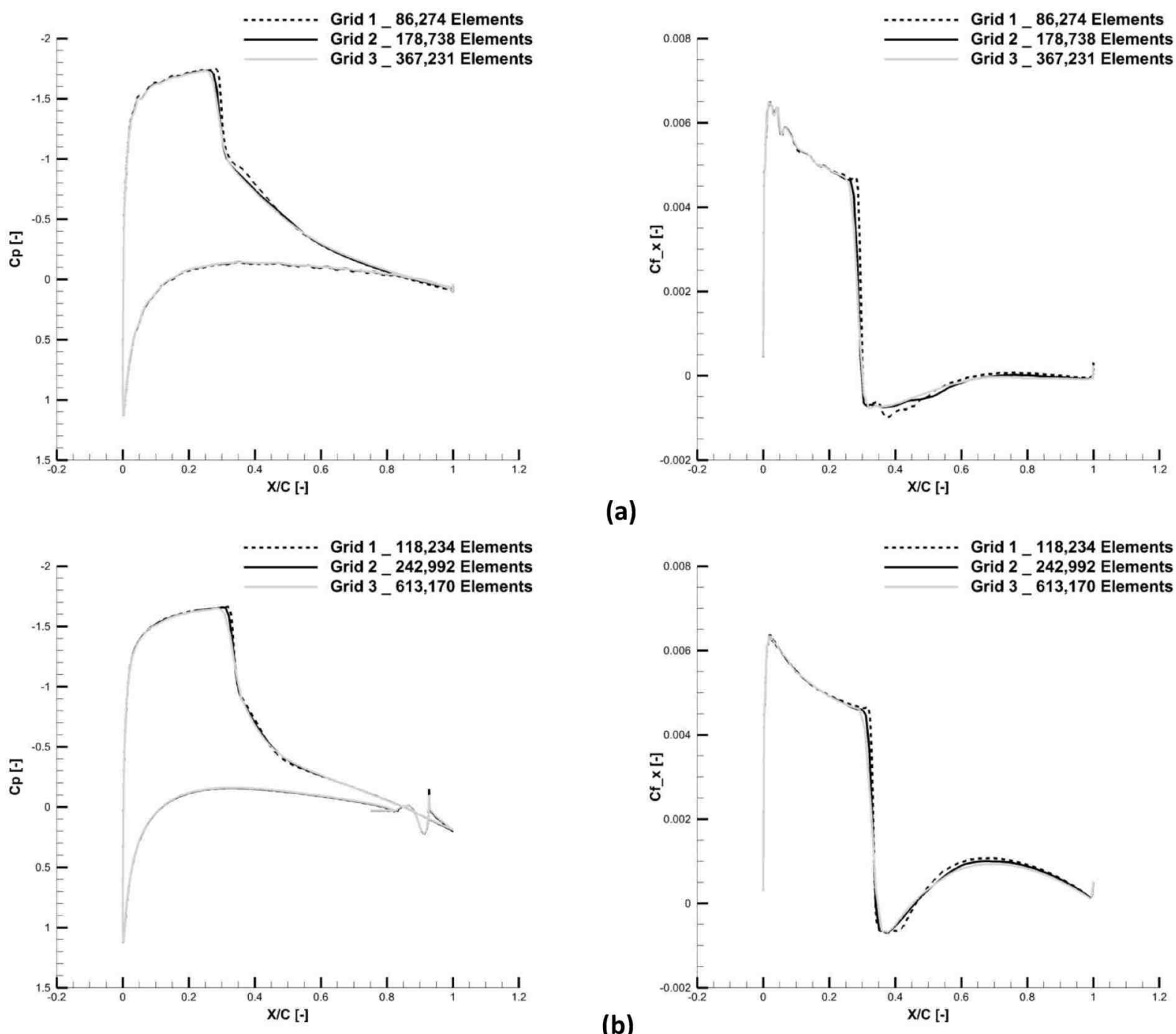

(a)

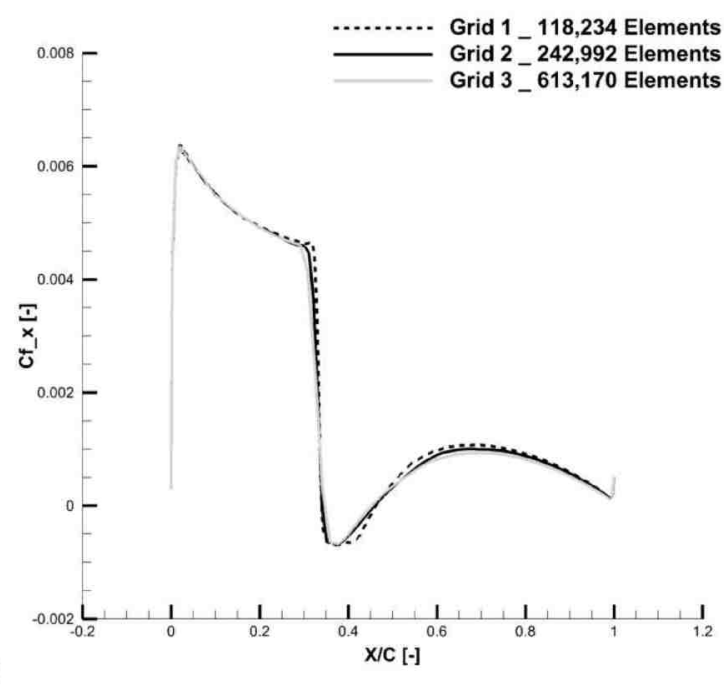

Figure 3.4 Grid convergence analyses. Test Case 1. (a) NACA0012 airfoil, (b) NACA0012 airfoil with $\mathrm{G}^{3} \mathrm{~L} 6$ modification, $M=0.7$ and $R e=9 \times 10^{6}$, angles of attack $4.74^{\circ}$. 


\subsubsection{Validation}

The results were compared to the experimental results of [88] and [89], i.e. to Test Cases 1 and 2 for the baseline airfoils. Excellent agreement was found by using the proposed mesh settings as shown in Figs. 3.5 and 3.6. Therefore, the selected numerical setup and parameters proved to be reliable enough to investigate the flow not only around the baseline airfoils, but also around the newly proposed modified airfoils in this study with some extra measurements as explained in the next section.
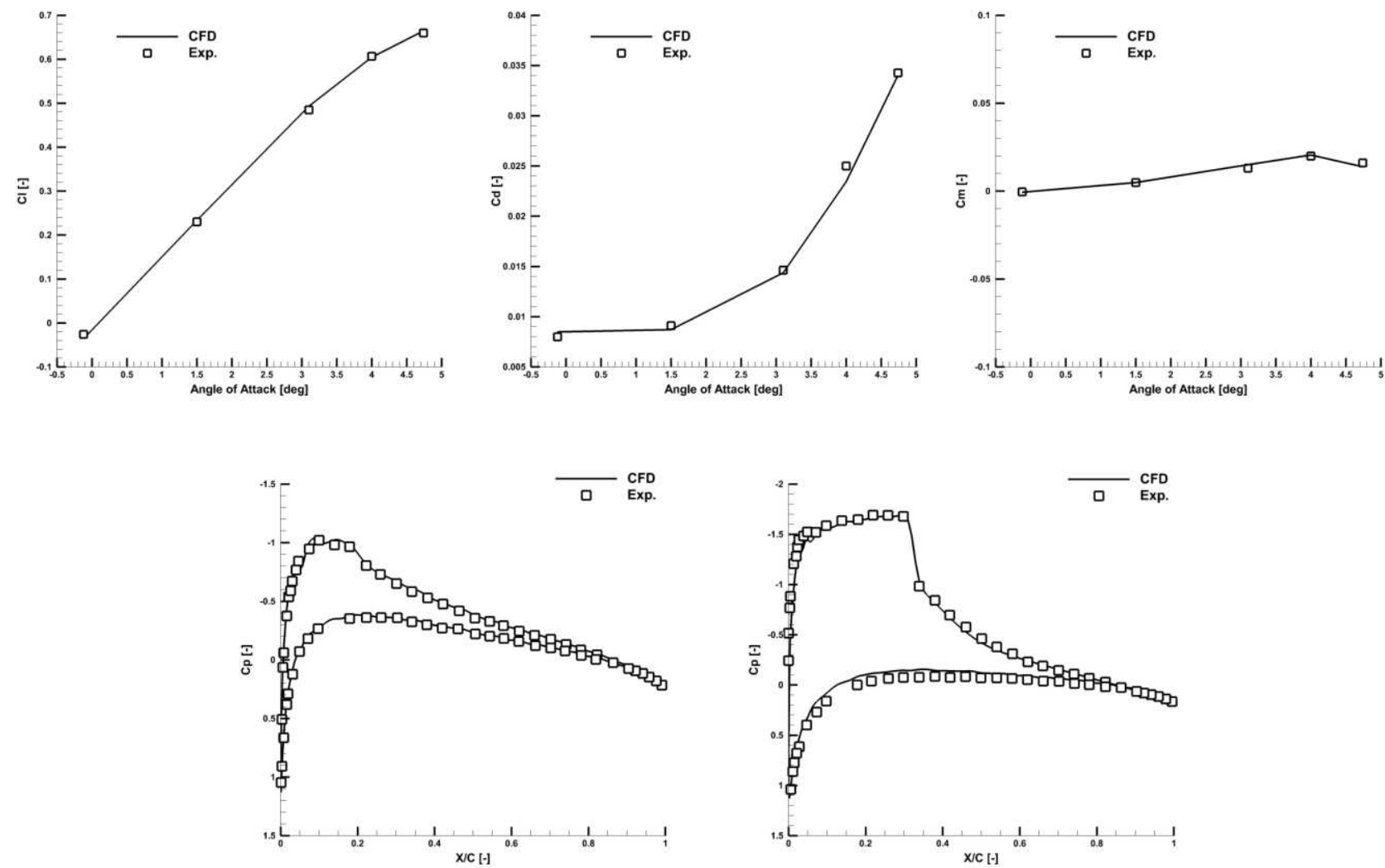

Figure 3.5 Validation of Test Case 1, NACA0012 airfoil, $M=0.7$ and $R e=9 \times 10^{6}$. First raw illustrates the aerodynamic results. Second raw illustrates the coefficient of the pressure distribution at angles of attack $1.5^{\circ}$ and $4.74^{\circ}$, respectively. 

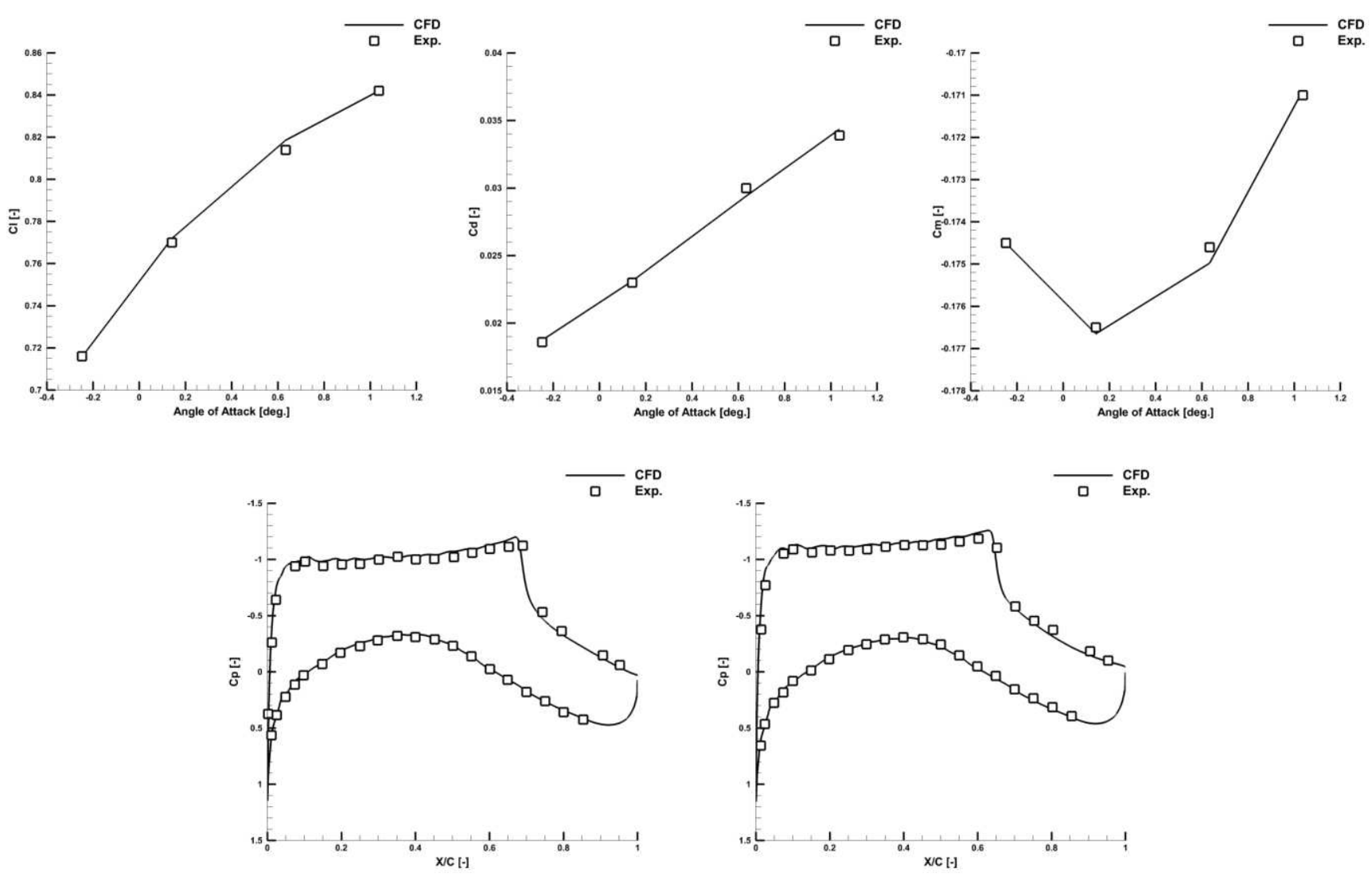

Figure 3.6 Validation of Test Case 2, NASA SC(3)-0712(B) airfoil, $M=0.78$ and $R e=$ $30 \times 10^{6}$, First row illustrates the aerodynamic results. Second row illustrates the coefficient of the pressure distribution at angles of attack $0.14^{\circ}$ and $1.04^{\circ}$, respectively.

\subsubsection{Simulation Precautions}

The proposed flow control concept is inspired by a BFS and as such, will involve geometry modifications via implementing a cavity on one of the airfoil surfaces. As will be seen later, the role of this cavity is to trap a vortex inside it. This raises two questions from computational point view: a) what mesh density should be employed inside the cavity to resolve this vortex properly? b) will the flow inside the cavity be steady or unsteady? 
In order to answer these questions, the following additional verification tests were conducted for transonic flows.

\section{A. Determining the minimum mesh density inside a cavity}

In order to determine the mesh density inside a cavity, verification and validation with 3 types of cavity flows was conducted. There are many investigations in the literatures regarding the transonic flow over the cavities. However, the current work is based on [8]. Cavities with length to depth ratio (L/D) in the range of $6 \leq \mathrm{L} / \mathrm{D} \leq 12$ were examined. These were an open cavity with length to depth ratio of $\mathrm{L} / \mathrm{D}=6$, a transitional cavity with $\mathrm{L} / \mathrm{D}=10$ and a closed cavity with $\mathrm{L} / \mathrm{D}=15$. All three cavities were simulated under two Mach numbers, 0.6 and 0.8 and Reynolds numbers per meter of $1.23 \times 10^{7}$ and $1.55 \times 10^{7}$, respectively. The computational domain and mesh is shown in Fig. 3.7.

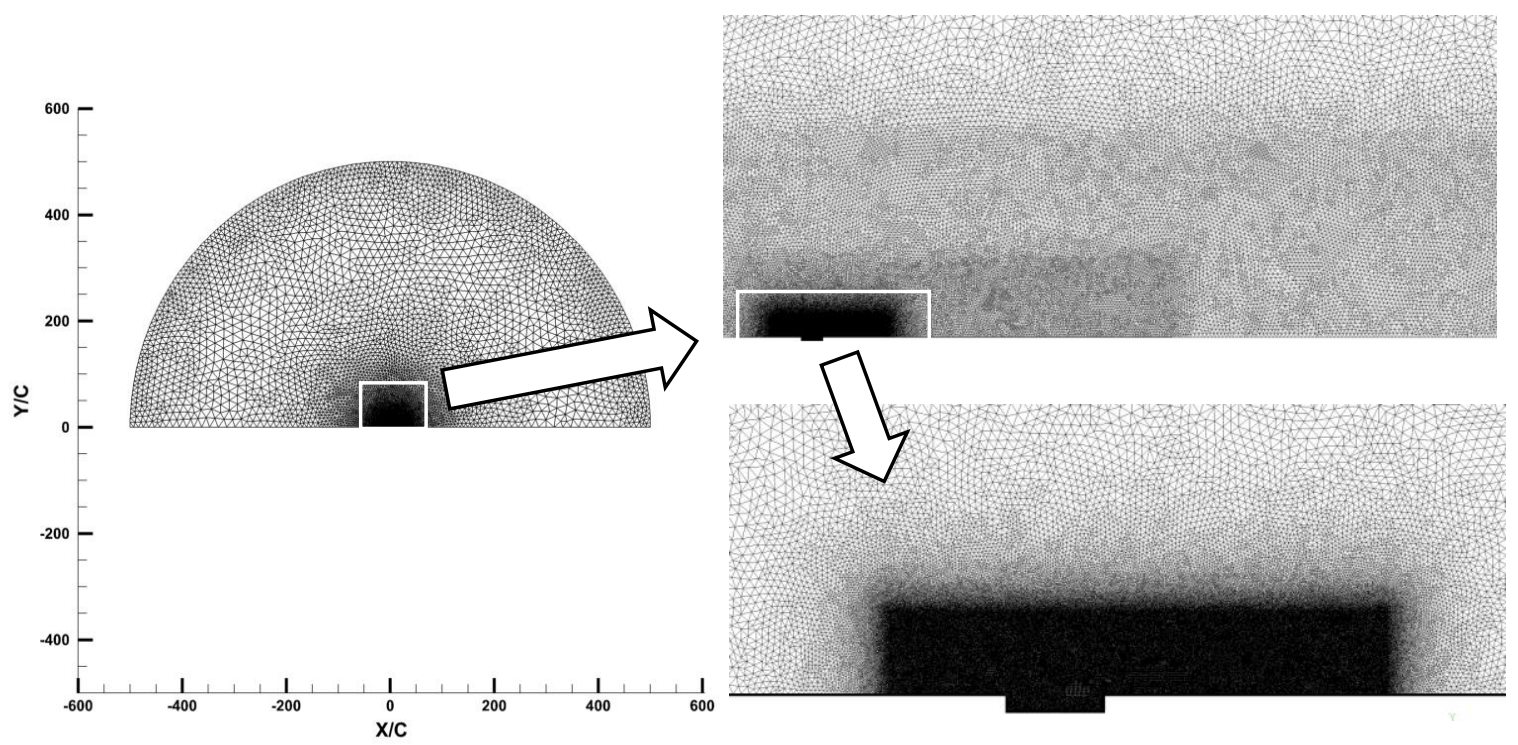

Figure 3.7 Computational domain and the mesh levels used in the transonic flow over rectangular cavity. 
The coefficient of pressure inside the cavities was selected as the parameter for the gird convergence study. Due to space constraint, only one example of the grid dependence study, along with the validation, is shown for the transonic flow over the cavity in Fig. 3.8.
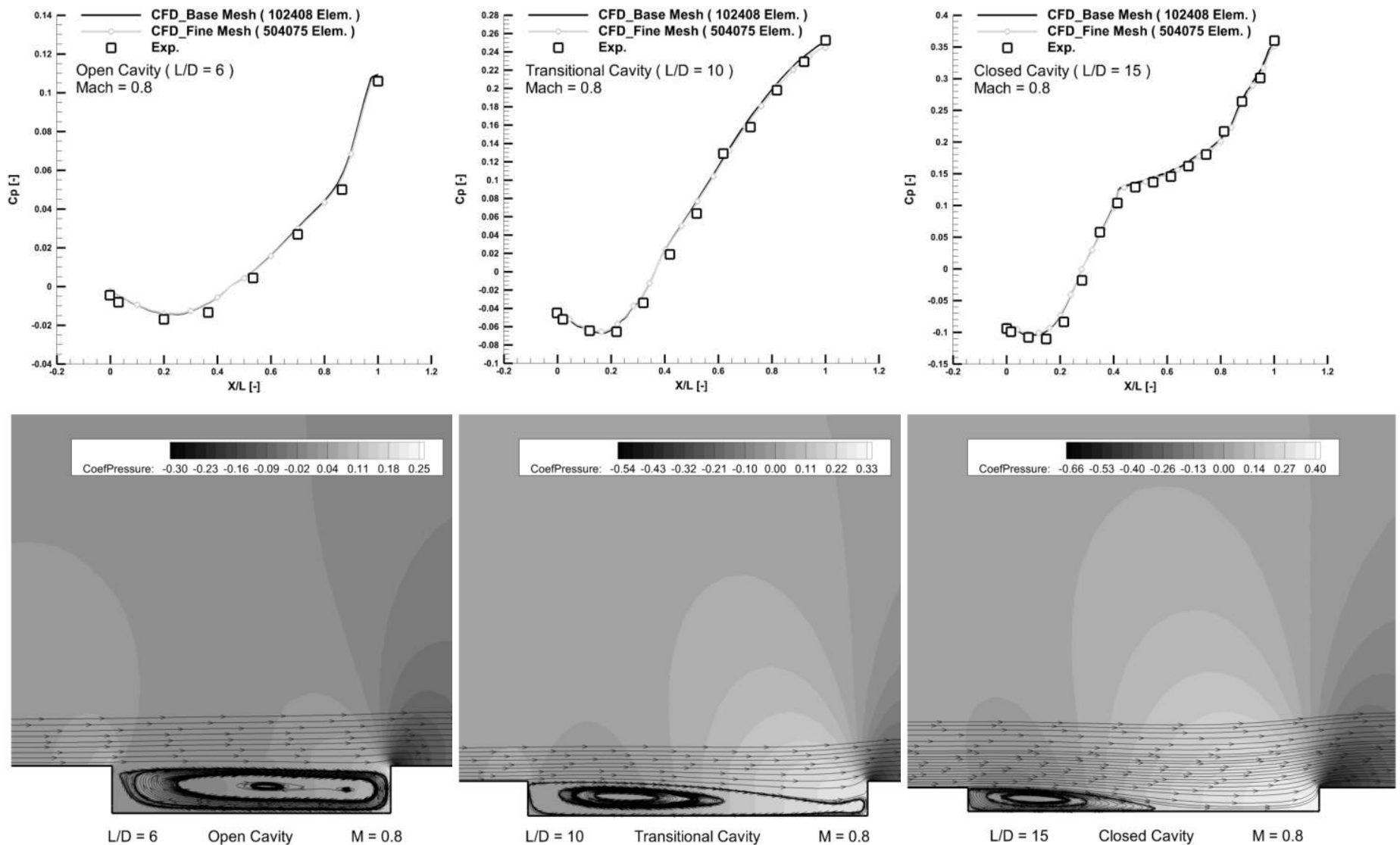

Figure 3.8 Verification and validation of one of [8] test Cases, $M=0.8$ and $R e / m=$

$$
1.55 \times 10^{7}
$$

As can be seen from Fig. 3.8, the baseline and fine meshes yielded identical results and as such, the baseline mesh characteristics (spacing at the wall, mesh density, etc.) were employed in all future cavity simulations. In Fig. 3.8, comparisons with the 
experimental data are shown, showing excellent validation of the proposed CFD methodology for cavities.

\section{B. Verification of the steady nature of flow inside the cavity}

In order to verify that the flow is steady inside the cavity, steady and unsteady simulations were conducted for an airfoil featuring one of the most complex cavity geometries proposed, a NACA 0012 airfoil with the $\mathrm{G}^{3} \mathrm{~L} 6$ modification. Note that the details of the surface modifications and their notations will be introduced later, in Chapter 4. At this point, only an illustration of the examined geometry is provided in Fig. 3.9.

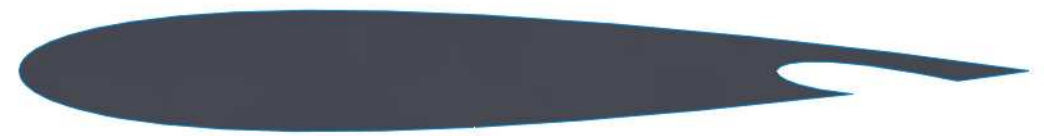

Figure 3.9 NACA 0012 with $\mathrm{G}^{3} \mathrm{~L} 6$ modification.

This test was conducted according to the following principles:

1. Two monitor points were created, one inside the cavity and one just after the shockwave to monitor the convergence of the solutions at each angle of attack, regardless of the residual limits of the continuity and energy equations. From Fig. 3.10 , one can clearly see that although the aerodynamic coefficients (drag in this case) reached their steady values approximately after 500 iterations, the two selected monitor points suggested that the solution is not yet converged. Therefore, the solution convergence for the modified airfoils was based on the convergence of the two selected monitor points for each following case. 
2. Since the proposed flow control technique might lead to unsteady flow in the “trapped vortex generator" region, time-dependent simulations (with $\Delta t=10^{-5} \mathrm{~s}$ ) were conducted at two typical angles of attacks representative of the fixed-wing and rotary-wing applications $\left(3.1^{\circ}\right.$ and $\left.4.74^{\circ}\right)$ using one of the most complicated design (NACA 0012 with the $\mathrm{G}^{3} \mathrm{~L} 6$ modification). Fig. 3.10 shows that the flow inside the cavity was actually steady and that there is no need to conduct an unsteady or transient simulation. Fig. 3.10 depicts the results of these tests.
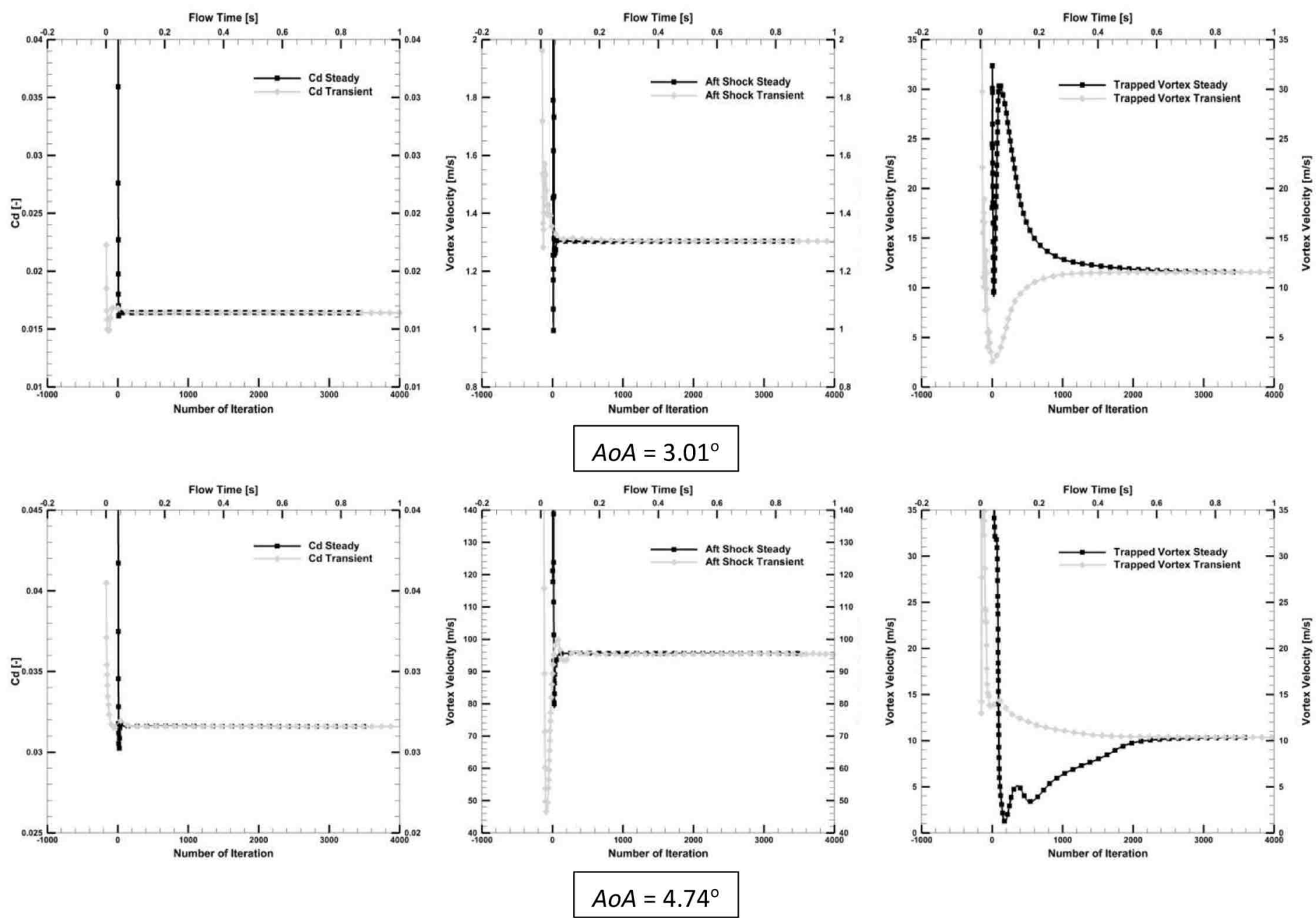

Figure 3.10 Time-independence study at the monitor points for Test Case 1, NACA 0012 with $\mathrm{G}^{3} \mathrm{~L} 6$ Modification at $A o A=3.01^{\circ}$ (first row) and $A o A=4.74^{\circ}$ (second row), at $M=0.7$ and $\operatorname{Re}=9 \times 10^{6}$. 


\subsection{D Constant Freestream Dynamic Stall}

\subsubsection{Test Cases}

The 2D dynamic stall test cases shown in this section were based on an experiment reported by McAllister et al. [93]. They represent flow conditions typical of dynamic stall occurring on a helicopter retreating blade in forward flight. The baseline NACA 0012 airfoil has a $0.61 \mathrm{~m}$ chord length and is subjected to freestream Mach number of 0.3 and chord-based Reynolds number of $3.76 \times 10^{6}$. The airfoil oscillates about the quarter chord as in the experiment and according to a sinusoidal motion described by the equation

$\alpha(t)=\alpha_{\text {mean }}+\alpha_{\text {amplitude }} \sin (\Omega t)$

in which the mean angle of attack $\left(\alpha_{\text {mean }}\right)$ was $15^{\circ}$ and the oscillation amplitude $\left(\alpha_{\text {amplitude }}\right)$ $10^{\circ}$. The reduced frequency $(k)$ for the current test case was 0.101 , where

$k=\Omega c / 2 U$

and $U$ is the velocity of the freestream. It is worth to mention that McAllister's experiment [93] had 1 pressure transducer on the airfoil nose, 16 on the upper surface and 10 on the lower surface. The collected data were reported to have an error by no more than \pm 0.2 for $C_{l}, \pm 0.10$ for $C_{d}$ and \pm 0.05 for $C_{m}$.

\subsubsection{Computational Domain}

A computational domain extending $500 c$ around the airfoil was employed (Fig. 3.11). Such size is quite unique since it has not been reported in the literature earlier (see CFD references provided in Chapter 2, Sections 2.5 and 2.6). Its use was prompted in an effort to provide a solid and accurate AoA oscillation that leads to robust simulation 
results, a domain design study was conducted to realize the best possible design. In the literature, the AoA oscillation is typically achieved either via a dynamic mesh, where only the airfoil is subjected to the oscillation with the mesh deforming accordingly [71, $84,85]$, or by oscillating the whole domain $[60,73]$. In the present work the domain was divided into two zones: a fixed one (ending $500 \mathrm{c}$ from the airfoil) and a rotating one, within which the airfoil was located (Fig. 3.11). The information exchanged between them via the interface rim was realized via FLUENT's sliding mesh technique [94]. It was found from the domain study above, that the sliding mesh approach outperforms the other techniques used in the literature as shown in Fig. 3.12, provided that the radius of the rotating domain is in the range of $10 c \leq R \leq 100 c$ to negate the interface effect on the flow around the airfoil. Hence, the radius of the rotating domain used in the current work was decided to be $100 c$.

The outer edges of the domain were set as pressure far field. In the absence of clear information about the turbulent intensity $(I)$ in the experiment of McAllister et al. [93], this parameter was selected to be $1 \%$, similar to that suggested by FLUENT's user guide [94] for external flow simulations. The airfoil surfaces were set to no-slip wall boundary conditions. 


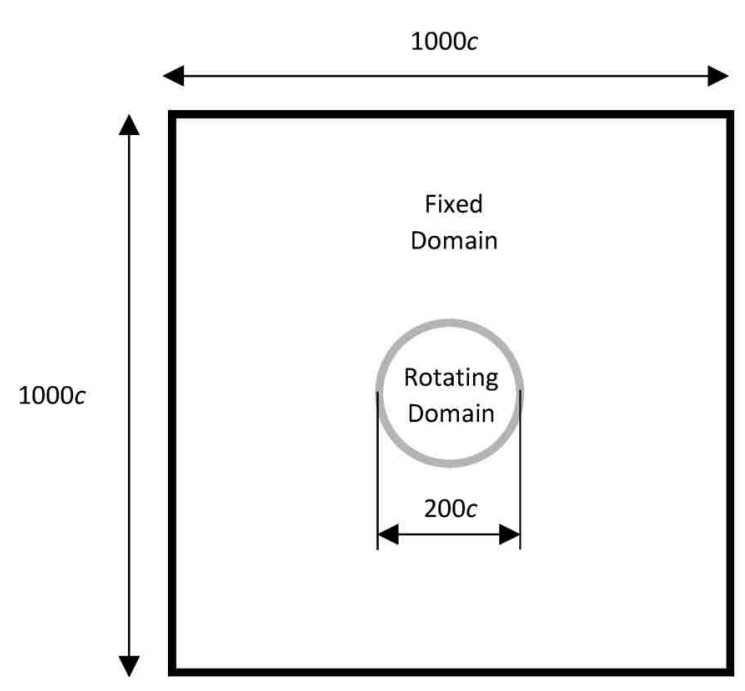

Figure 3.11 Computational domain sketch, the "black" boundaries represent the pressure far field and the "gray" boundaries are the mesh interface.

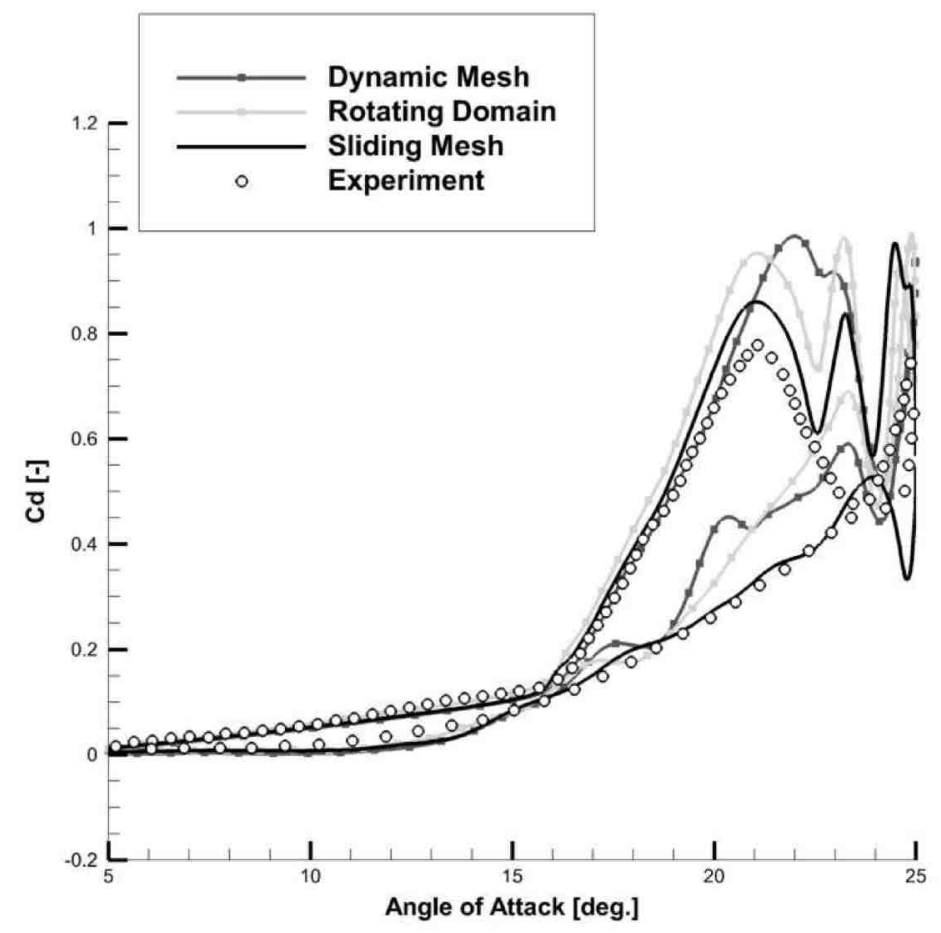

Figure 3.12 Domain design study results representation using drag coefficient, (SST $k$ -

$\omega)$. NACA $0012, M=0.3, \operatorname{Re}=3.76 \times 10^{6}$ and $\alpha=15^{\circ} \pm 10^{\circ}$. 


\subsubsection{Mesh Generation}

The computational domain was divided into cells via a hybrid structuredunstructured mesh, consisting of a total of 192,676 cells. A structured mesh consisting of rectangular cells was used only in the "inflation layer" around the airfoil, so that the boundary layer can be effectively captured. An unstructured mesh consisting of 2D triangles was used outside of this inflation layer. The unstructured mesh was refined in the area above and downstream of the airfoil, where the dynamic stall vortices are expected to appear (Fig. 3.13 b). 650 cells were distributed along the airfoil surface. 40 layers of structured mesh were employed inside the inflation layer, with the first spacing above the wall kept around $y^{+}=1$ since no wall function was used in the turbulence model (Fig. $3.13 \mathrm{c})$.

\subsubsection{Initialization}

The initialization of the transient simulations in this study was accomplished by generating an initial steady-state simulation, in which the airfoil was set to the minimum angle of attack of $5^{\circ}$, for which the flow is still attached on the airfoil surface. The steadystate simulation was run until the residuals of the continuity and energy equations reached $10^{-9}$ convergence level. This approach allowed to eliminate the initial transient of the unsteady simulations, i.e. to achieve periodic results for the integral aerodynamic coefficients after only one cycle. This approach saves lot of computational time and to the knowledge of the author, no other work has used it before. Hence, this is another original contribution of this thesis. Fig. 3.14 illustrates the time histories of the aerodynamic loads for 5 cycles, illustrating repeatability in the first cycle itself. 


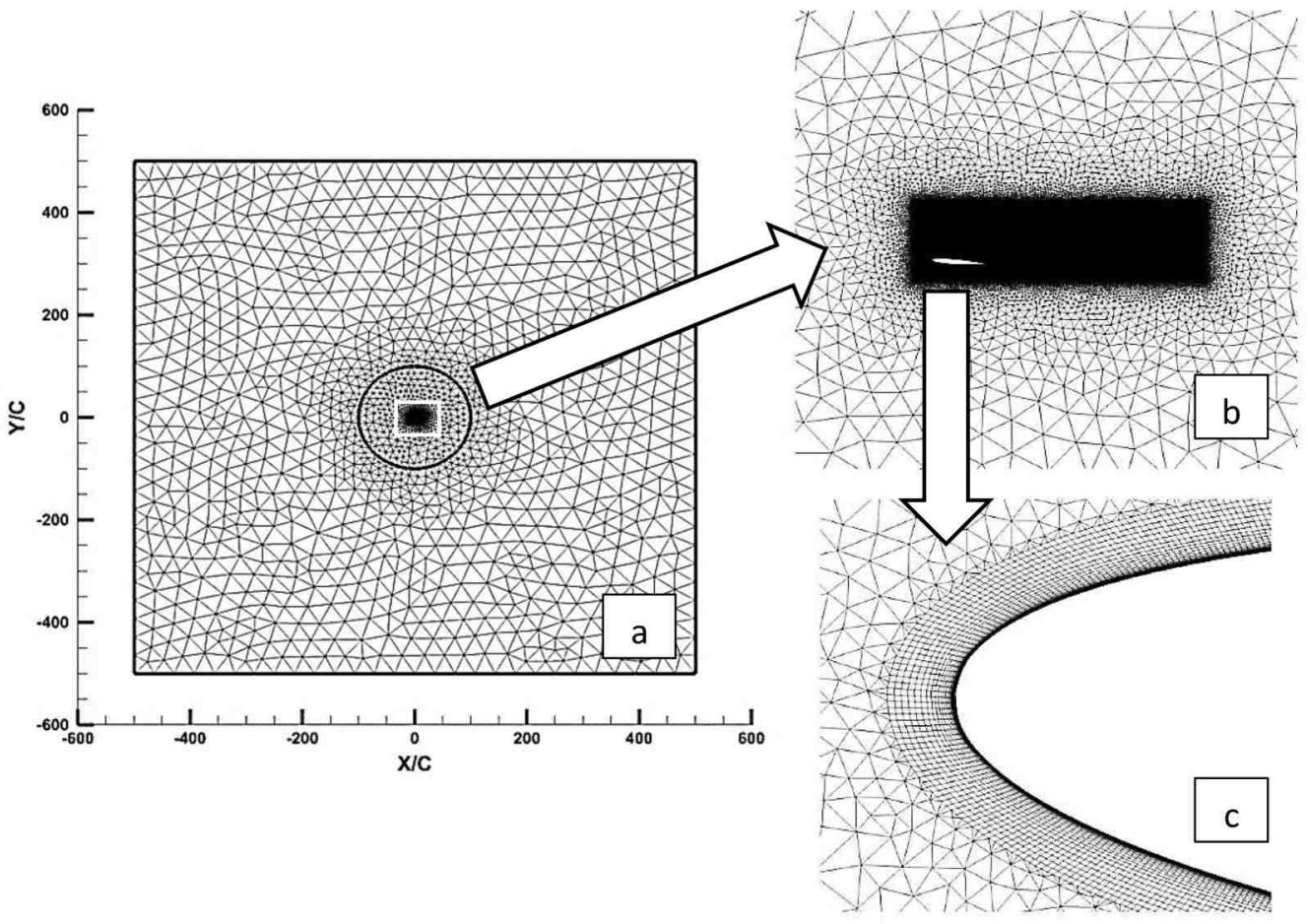

Figure 3.13 (a) Computational domain mesh, (b) refined mesh area inside the rotating domain and (c) inflation layers and cell distribution around airfoil surface.
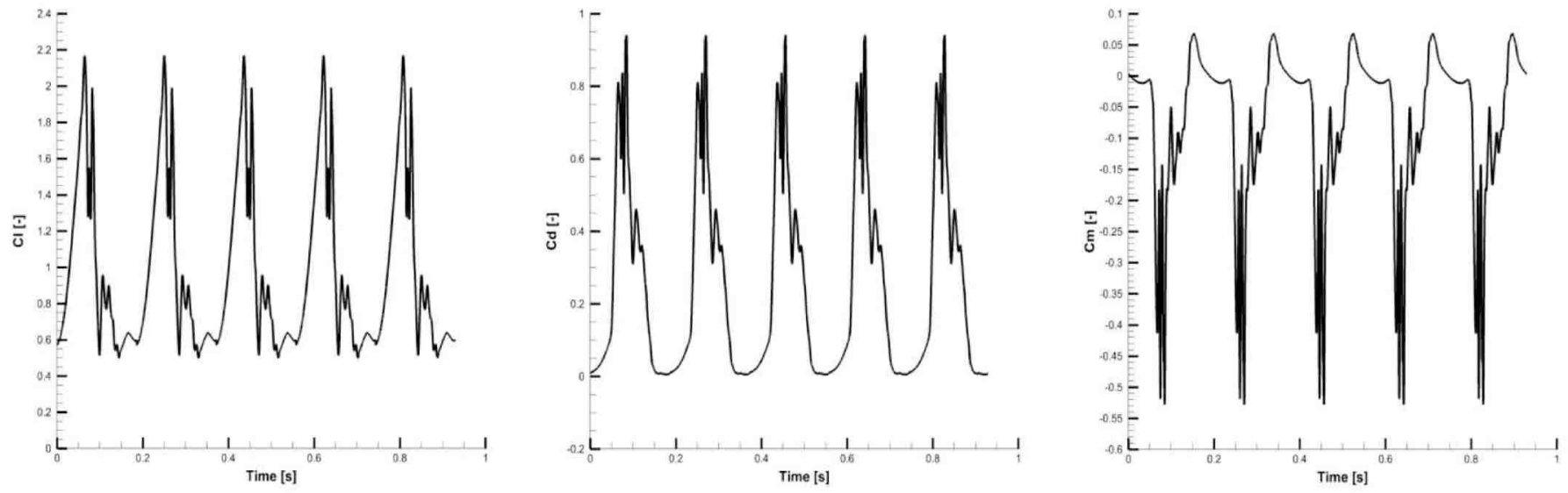

Figure 3.14 Time history of the aerodynamic loads for steady freestream dynamic stall simulation (SST $k-\omega)$. NACA 0012, $M=0.3, R e=3.76 \times 10^{6}$ and $\alpha=15^{\circ} \pm 10^{\circ}$. Note the lack of initial transient, i.e. the periodicity of the curves immediately in the $1^{\text {st }}$ cycle after start-up. 


\subsubsection{Verification}

Grid-dependence, time step dependence, Courant number dependence and residual dependence tests were performed for the baseline simulations. Due to space constraints, only the lift graphs from each test will be presented in this thesis. However, the end result of the verification was that the baseline mesh density of 192,676 cells and 1,600 time-steps per cycle shall be used with the Courant number (CFL) of 200, because in the implicit dual time method used here, CFL is not linked to the physical time-step, rather than that, it is linked to fictitious time-step. This required 20-30 iterations between each consecutive real time-step in the unsteady simulations to reach a residual of $10^{-3}$. Further refinement of these parameters did not improve the accuracy of the results any further, as shown in Figure 3.15.

\subsubsection{Validation}

The numerical method was validated for the baseline case, i.e. the NACA 0012 airfoil exposed to a constant freestream of Mach 0.3 and $R e=3.76 \times 10^{6}$ and undergoing a $15^{\circ} \pm 10^{\circ}$ pitch oscillation. Computational results were compared to the experimental data of McAllister et al. [93]. Fig. 3.16 shows the results of the validation for both the SA and SST $k$ - $\omega$ turbulence models, illustrating a very good agreement between CFD and experiment for both the SA and SST $k$ - $\omega$ turbulence models.

It is very important to draw the attention towards the two peaks in in all three parameters around the maximum angle of attack shown in Fig. 3.16b. It is not clear 
whether these peaks appear due to the use of the SST $k-\omega$ turbulence model in the simulations [85-87] or they were originally part of the experiment.
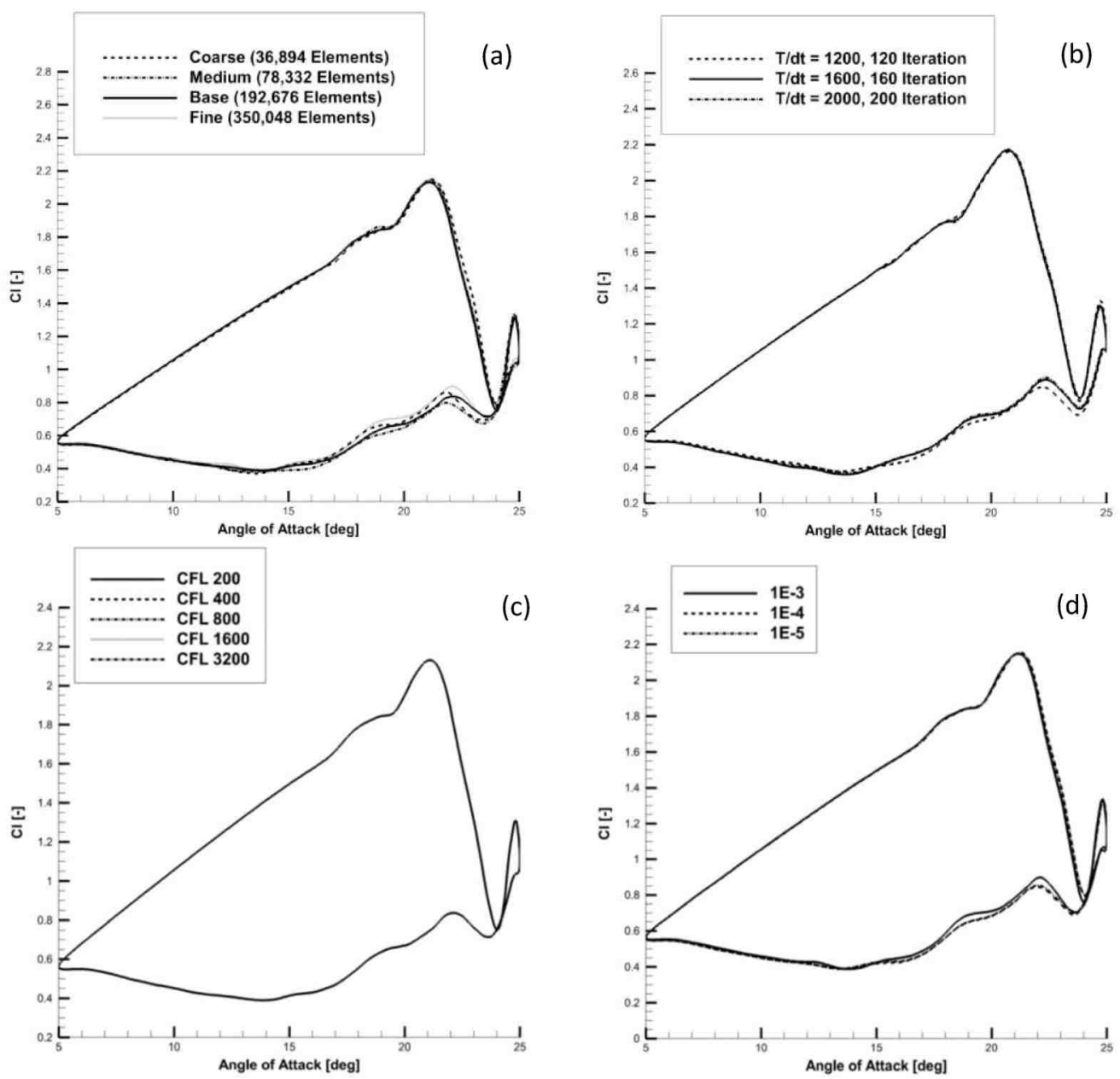

Figure 3.15 Verification tests, (a) grid -dependence, (b) time step dependence, (c) CFL number and (d) residual dependence. All using (SA). NACA 0012, $M=0.3, R e=$

$$
3.76 \times 10^{6} \text { and } \alpha=15^{\circ} \pm 10^{\circ} \text {. }
$$

McAllister et al. [93] reported an unusual large number of random irregularities, when conducting the experiment using the NACA 0012 airfoil for the same test case as in 
this study, except that $\alpha_{\text {mean }}=10^{\circ}$ and $\alpha_{\text {amplitude }}=5^{\circ}$. These irregularities were eliminated from the experimental report by using linear interpolation for the experimental data.

As a result, the peaks might be a phenomenon related to NACA0012 airfoil itself. If it was the SST $k-\omega$ model, then [84] concluded that the influence of turbulence modeling is test case dependent, and perhaps the current case needs to account for boundary layer transition by using a transitional model instead of the fully turbulent model used in this study.
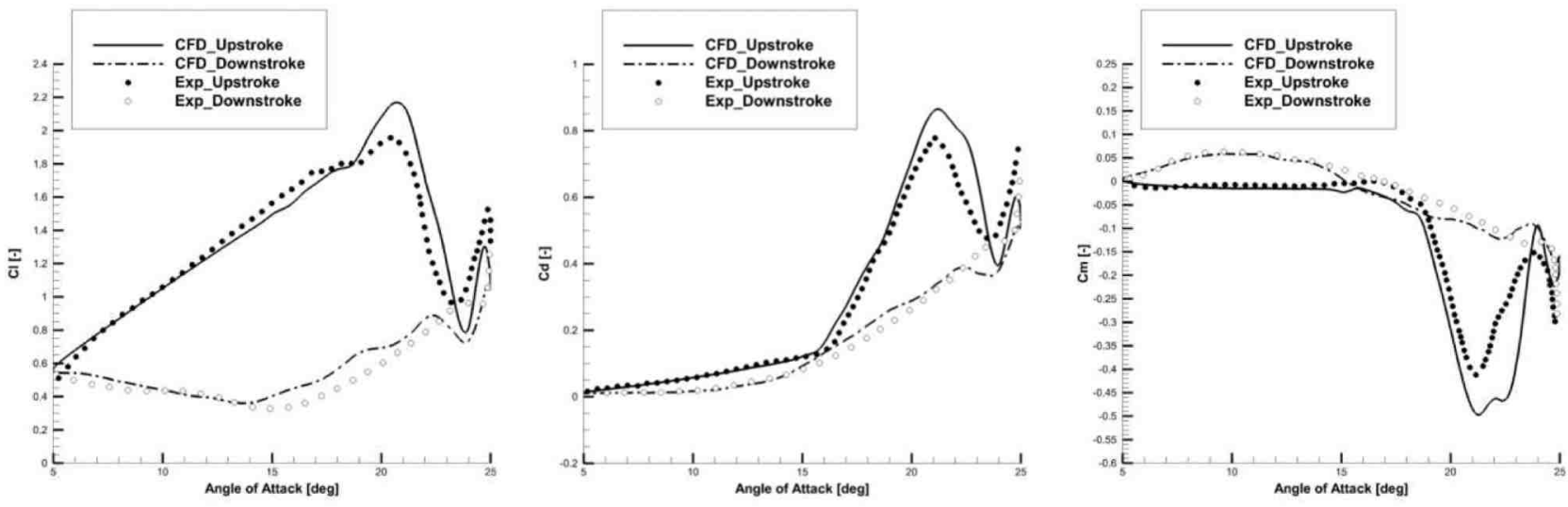

a
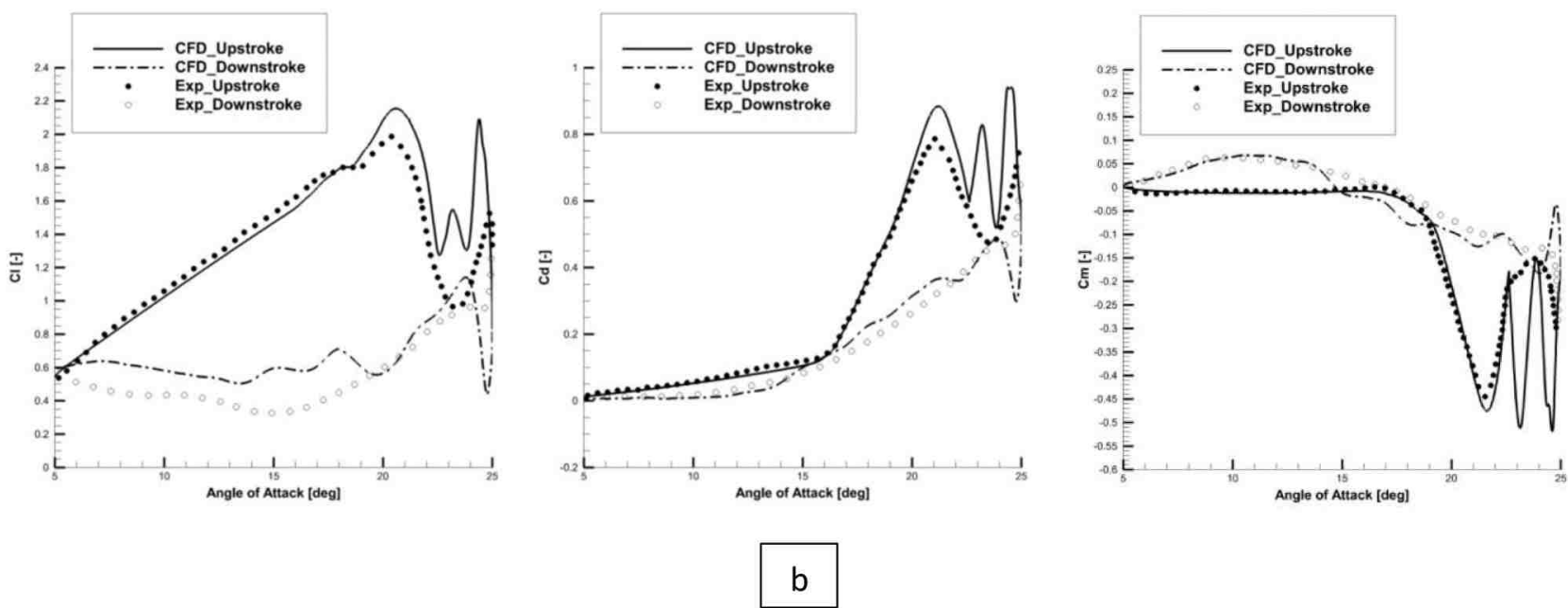

Figure 3.16 Validation of the simulation results for (a) SA turbulence model and (b) SST $k$ - $\omega$ turbulence model. NACA 0012. $M=0.3 . R e=3.76 \times 10^{6}$ and $\alpha=15^{\circ} \pm 10^{\circ}$. 


\subsection{D Fluctuating Freestream Dynamic Stall}

\subsubsection{Test Cases}

To provide realistic flight conditions as much as possible, the present study also considered the conditions of UH-60A Blackhawk helicopter in fast forward flight. Table 3.2 lists the simulation parameters and conditions. The operating parameters of UH-60A as well as the flight conditions were based on references [95-98]. The simulation conditions were chosen to be similar to that in Coleman et al. [99] (which is also based on UH-60A flight tests). Therefore, an advance ratio of 0.33 at radius $r / R=0.865$ of the main rotor radius was selected.

Table 3.2 Simulation parameters and conditions.

\begin{tabular}{lcll}
\hline \hline UH-60A parameters [95-98] & Simulation conditions [98-99] & \\
\hline Main Airfoil Chord & $0.53 \mathrm{~m}$ & Tip Mach number & 0.621 \\
Rotational Speed & $26.69 \mathrm{rad} / \mathrm{s}$ & Flight Mach number & 0.205 \\
Rotational frequency & $4.25 \mathrm{~Hz}$ & Local Mach number at $\mathrm{r} / \mathrm{R}=0.865$ & 0.537 \\
Tip speed & $218.25 \mathrm{~m} / \mathrm{s}$ & Local $R e$ at $\mathrm{r} / \mathrm{R}=0.865$ & $6.1 \times 10^{6}$ \\
\hline \hline
\end{tabular}

Regarding the angle of attack variation, Kerho [71] based on Bousman [100], predicted the blade pitching history of UH-60A using the comprehensive rotorcraft analysis code CAMRAD II at advance ratio of 0.33 and radius $r / R=0.865$. According to Kerho [71], a sinusoidal function of $10^{\circ}-10^{\circ} \sin (\Omega t)$ can be viewed as an acceptable approximation for the effective angle of attack history and was therefore used in the current study as well (Fig.3.17). 


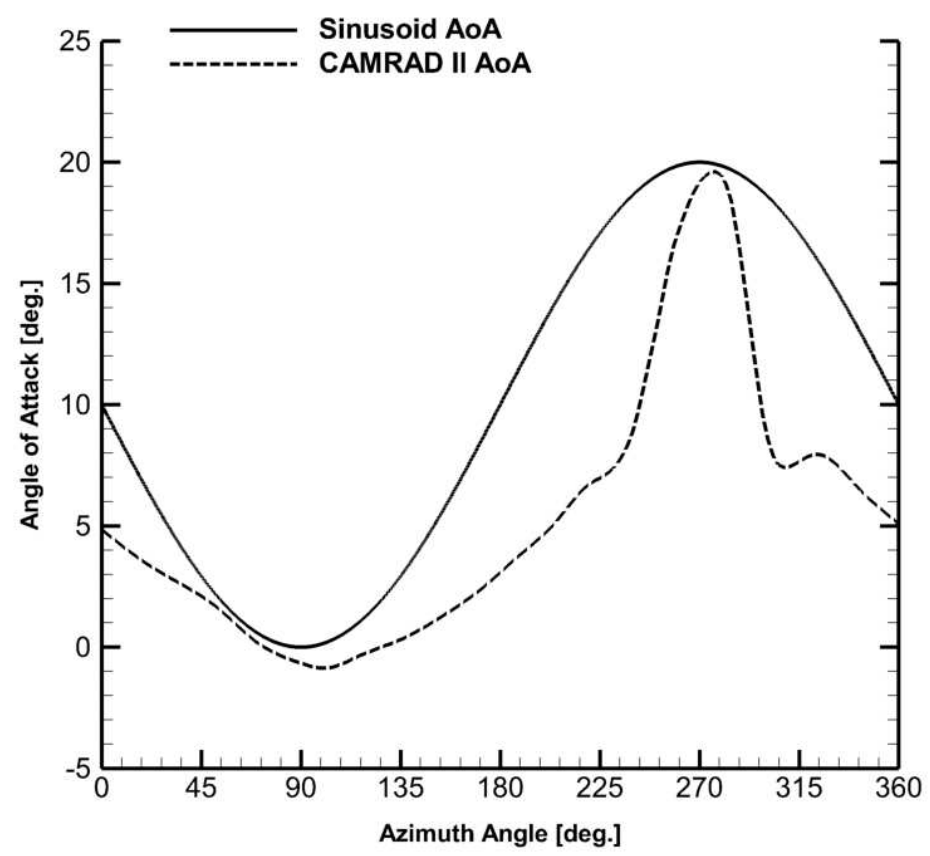

Figure 3.17 CAMRAD II vs Sinusoid AoA variation for UH-60A. Forward $\mathrm{M}=0.205$, $\mathrm{r} / \mathrm{R}=0.865$ and advance ratio $\mu=0.33[71]$.

According to Table 3.2, the Mach number fluctuation shall be set to $0.537 \pm 0.205$. Equations (3.3) and (3.4) provide the formulation of the angle of attack oscillation and Mach number fluctuation, while Fig. 3.18 illustrates their resulting combination.

$$
\begin{aligned}
& \alpha(t)=10-10 \cos (\Omega t) \\
& M(t)=0.537-0.205 \cos (\Omega t+\pi)
\end{aligned}
$$

Note that there is a phase difference of $180^{\circ}$ between the periodic fluctuation of the freestream Mach number and the angle of attack. 


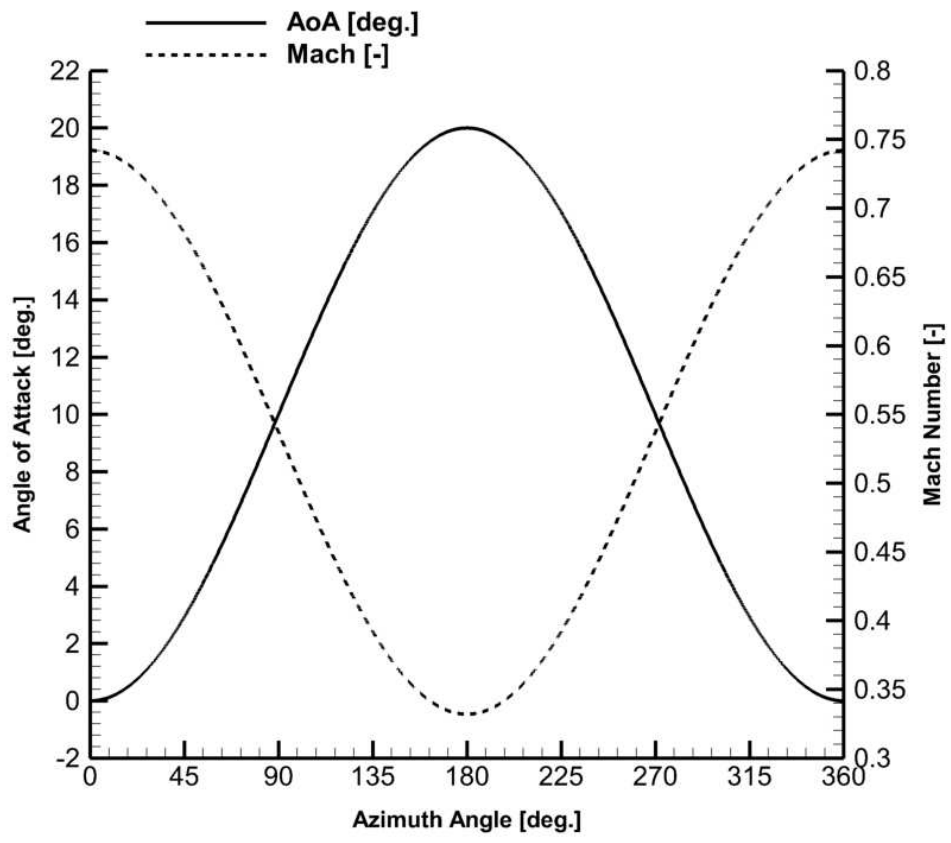

Figure 3.18 Freestream Mach number fluctuation versus angle of attack oscillation as a function of the azimuth angle.

\subsubsection{Computational Domain}

The domain and mesh developed in Sub-section 3.4.2 for constant freestream dynamic stall were adopted for the current, fluctuating freestream study. The novelty is the addition of the fluctuating freestream by setting the freestream at the inlet to Mach $0.537 \pm 0.205$. It was anticipated that this case would yield transonic flow at the extreme of the Mach number and thus it offers the possibility to examine the effects of transonic flow on dynamic stall.

Introducing a fluctuating freestream velocity simultaneously with the airfoils' oscillation can be achieved either by producing some form of velocity vector changes with time [60] or by a significantly more complicated procedure [71, 79]. Table 3.3 summarizes the differences between the current method and those used in the literature. 
Table 3.3 CFD literatures for fluctuating freestream dynamic stall in forward flight.

\begin{tabular}{cccc}
\hline \multicolumn{1}{c}{ References } & Inlet Location & \multicolumn{1}{c}{ Methodology } \\
\hline \hline Gosselin et al. [60] $10 c \quad$ & $\begin{array}{l}\text { C-type domain, in which the entire domain oscillates to provide } \\
\text { the angle of attack variation, while at the same time, the velocity } \\
\text { fluctuates at the inlet. }\end{array}$ \\
Kerho [71] & N/A & $\begin{array}{l}\text { C-type domain, in which the angle of attack oscillation is } \\
\text { achieved via grid deformation, while the freestream fluctuation is } \\
\text { accomplished by modifying the solver code to create an effect of } \\
\text { the body translating in the direction of the freestream, which } \\
\text { produces an additive velocity to the freestream. }\end{array}$ \\
Zhao et al. [79] & $\begin{array}{l}\text { Two embedded domains, with an inner C-type domain and an } \\
\text { outer rectangular domain. Velocity fluctuation is achieved via a } \\
\text { moving-embedded grid method where the airfoil is moving } \\
\text { periodically back-and-forth along the direction of the mean } \\
\text { freestream velocity, and at the same time, the angle of attack } \\
\text { oscillation is also achieved by periodically oscillating the airfoil. }\end{array}$
\end{tabular}

The methodology proposed in this work does not involve mesh deformation nor does not need to consider extra measures for the velocity vector changes, i.e. appears to be simpler and faster.

\subsubsection{Computational Domain Reconfiguration}

The computational domain was reconfigured to enable a fluctuating freestream simulation. Instead of using the pressure far field boundary condition, an inlet boundary condition was introduced at the left side of the domain, pressure outlet for the right side, while the upper and lower sides were set to be free-slip surfaces as shown in Fig. 3.19 This modification would provide a one directional flow for the fluctuating velocity, i.e. no change in the direction of the vectors was needed. 
An in-depth analysis to the physics of the fluctuating freestream at the inlet suggested that using the domain of Sub-section 3.4 .2 (inlet at $500 c$ or $265 \mathrm{~m}$ from the airfoil) is too large because the wave length of the velocity fluctuation is merely a 44.45 $\mathrm{m}$, i.e. for the velocity fluctuation to reach the airfoil, as many as 6 cycles are needed and this would imply too high computational cost. Therefore, based on the mean velocity, the inlet was proposed to be located at only $25 \%$ of the wave length $(\approx 18.9 \mathrm{c}$ in this case). It is also worth to mention that due to the inlet fluctuation conditions, the acoustic waves might also be propagated through the domain along with the velocity propagation.

As a result, the sizes of the domain from Sub-section 3.4.2 were modified to bring the inlet location to $20 c$, a distance, which proved to be the minimum sufficient inlet distance for a typical 2D CFD simulation [101].

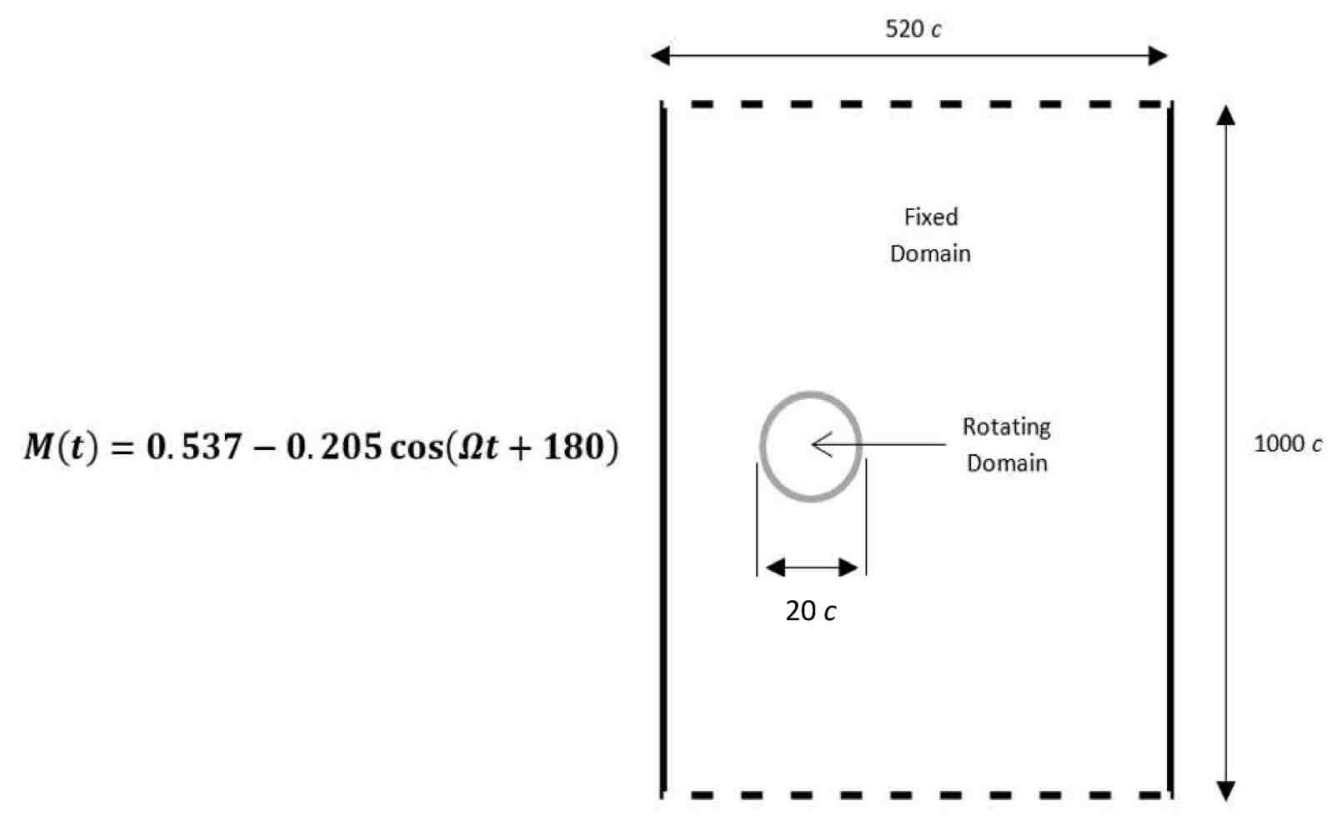

Figure 3.19 Computational domain sketch after the reconfiguration, the "black" lines represent the inlet and outlet boundaries with inlet to the left, the "dashed" lines were set to free-slip boundaries and the "gray" boundaries are the mesh interface. 


\subsubsection{Computational Time Delay}

Because changes at the inlet boundary take time to propagate to the airfoil, the resulting velocity profile at the airfoil location was in a phase delay relative to the inlet. Thompson $[102,103]$ suggested a solution for time-dependent boundary conditions (the inlet in this case), which is capable of addressing the time-difference behind this phasedelay accurately and it is given as:

$$
t_{d}(t)=\frac{20 c}{U(t)+a}
$$

Where $t_{d}$ is the time delay in seconds at each instantaneous velocity $U(t)$ and $a$ is the speed of sound. Once the time delay is calculated, the maximum time will be selected (usually at the minimum velocity for a uniform velocity profile) and then it will be implemented in the equations of motion. It was found that adding the time delay to equation (3.4) would increase the complexity of the simulation. Therefore, the best solution was to modify equation (3.3) to accommodate the time shift, leading to:

$$
\alpha(t)=10-10 \cos \left(\Omega\left(t-t_{d \max }\right)\right)
$$

Fig. 3.20 provides a detailed presentation for the velocity profiles and the final angle of attack and Mach number at the airfoil location. As seen in the figure, the intended formulation illustrated previously in Fig. 3.18 was successfully achieved. 

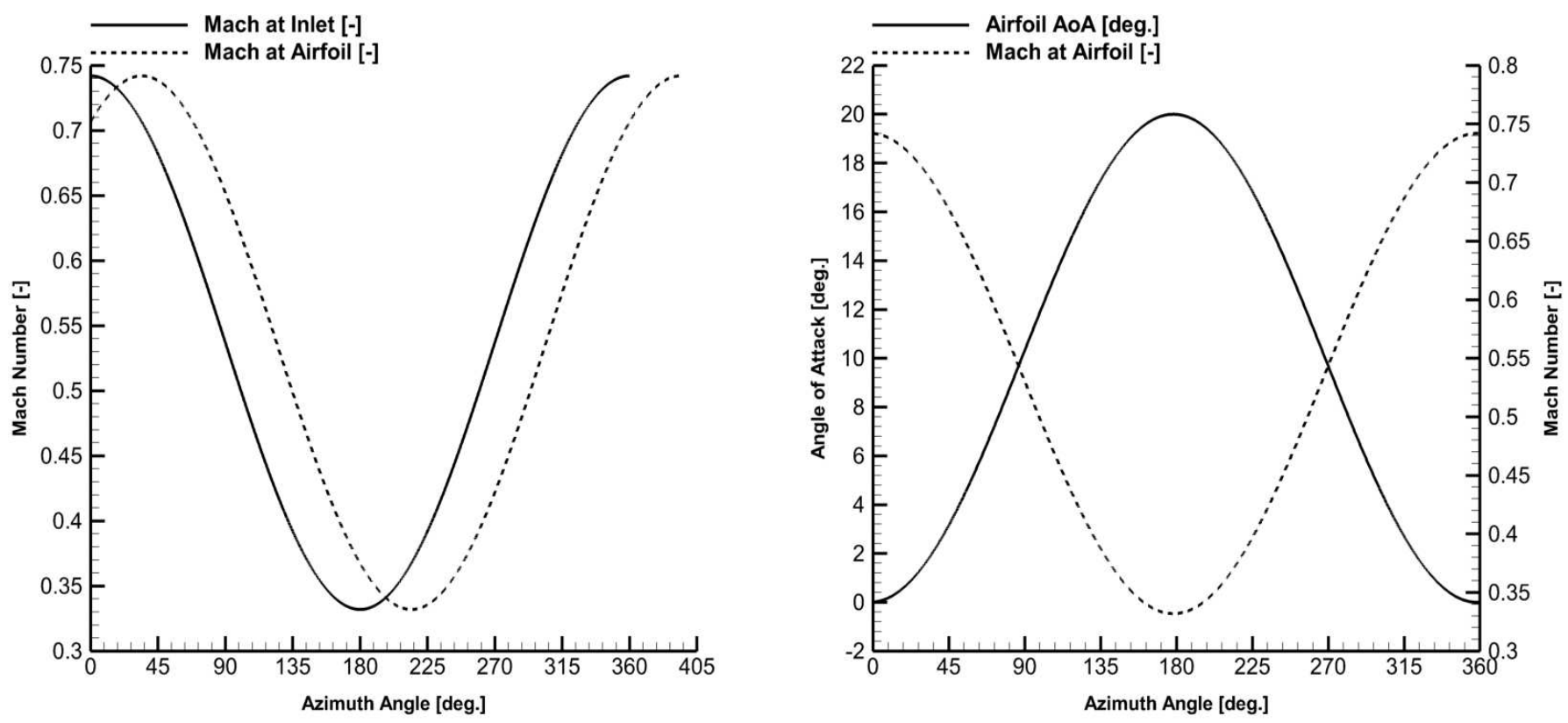

Figure 3.20 Mach number at the inlet and airfoil location (left) and the final Mach-AoA formulation used in the simulation (right).

\subsubsection{Mesh Generation}

The computational mesh was of a hybrid structured-unstructured type, with a total number of cells of 252,321. 650 cells were distributed along the surface of the airfoil. A structured rectangular mesh was used in the "inflation layer" around the airfoil, so that the boundary layer can be effectively captured. 40 layers of structured mesh were employed inside the inflation layer, with the first spacing around the wall kept well below $y^{+}=1$. Therefore, no wall function was used in the turbulence model. An unstructured mesh of 2D triangles was used everywhere outside of the inflation layer. The unstructured mesh was further refined in the area above and downstream of the airfoil as 
shown in Fig. 3.21, where the transonic shockwave and dynamic stall vortices are expected to appear.

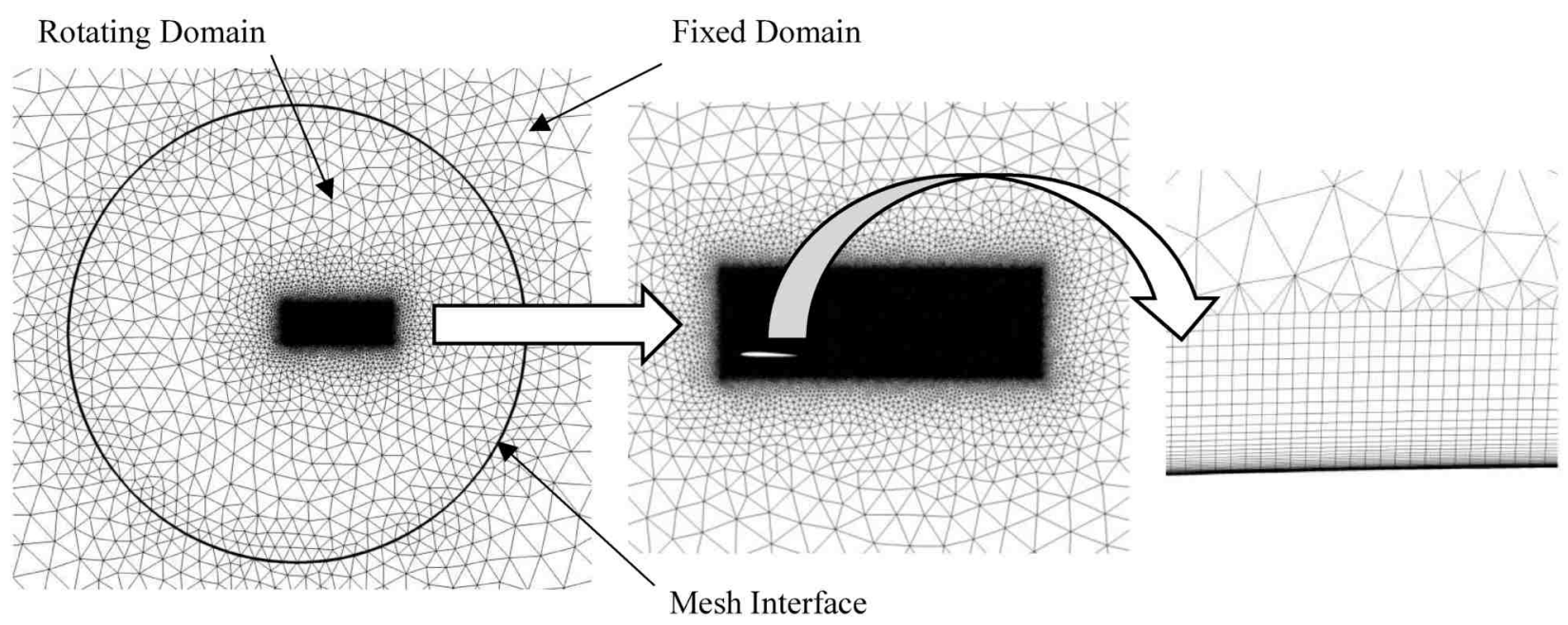

Figure 3.21 Magnified images of the mesh used in dynamic stall simulation under

fluctuating freestream.

\subsubsection{Initialization}

The initialization of the transient simulations was accomplished following the technique described in Sub-section 3.4.4. This meant that an initial steady-state simulation was generated with the airfoil set to the conditions corresponding to 0 degrees azimuth angle in Fig. 3.20, i.e. the minimum angle of attack and maximum Mach number. The steady-state simulation was run until the residuals of the continuity and energy equations converged to a level of $10^{-12}$. Then, the fluctuating freestream boundary condition was introduced in a fashion discussed above. Fig. 3.22 illustrates the time histories of the aerodynamic loads for 3 cycles. Note that with the help of the steady-state flow initialization, no initial transient is needed, periodicity can be observed directly from 
the $1^{\text {st }}$ cycle. Thus, following the methodology of this work, only one cycle is enough to simulate the fluctuating freestream dynamic stall.
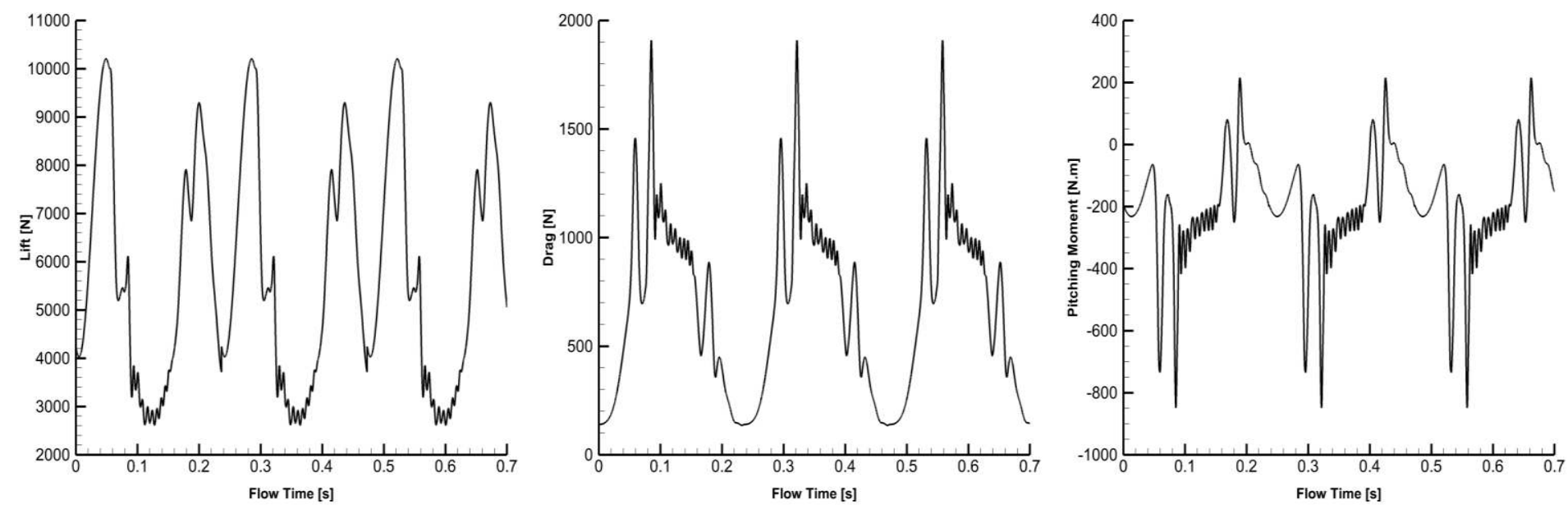

Figure 3.22 Time history of the aerodynamic loads for fluctuating freestream dynamic stall simulation. $\mathrm{SC1095,} M=0.537 \pm 0.205, R e=6.1 \times 10^{6}$ and $\alpha=10^{\circ} \pm 10^{\circ}$.

\subsubsection{Verification}

To increase the confidence in the above methodology, it was important to conduct a new series of grid dependence and other simulation parameters dependence studies to see if the modified domain and mesh was appropriate for a fluctuating freestream dynamic stall simulation. This was necessary because the inlet is now closer to the airfoil (20c) and the radius of the rotating domain was decreased from $100 c$ to only $10 c$. Timestep independence was achieved by using 2000 time-steps per pitching cycle and 100 inner iterations with a Courant number of 200 and a residual of $10^{-4}$ between each consecutive time-step. Fig. 3.23 shows the results of this grid convergence analysis. 

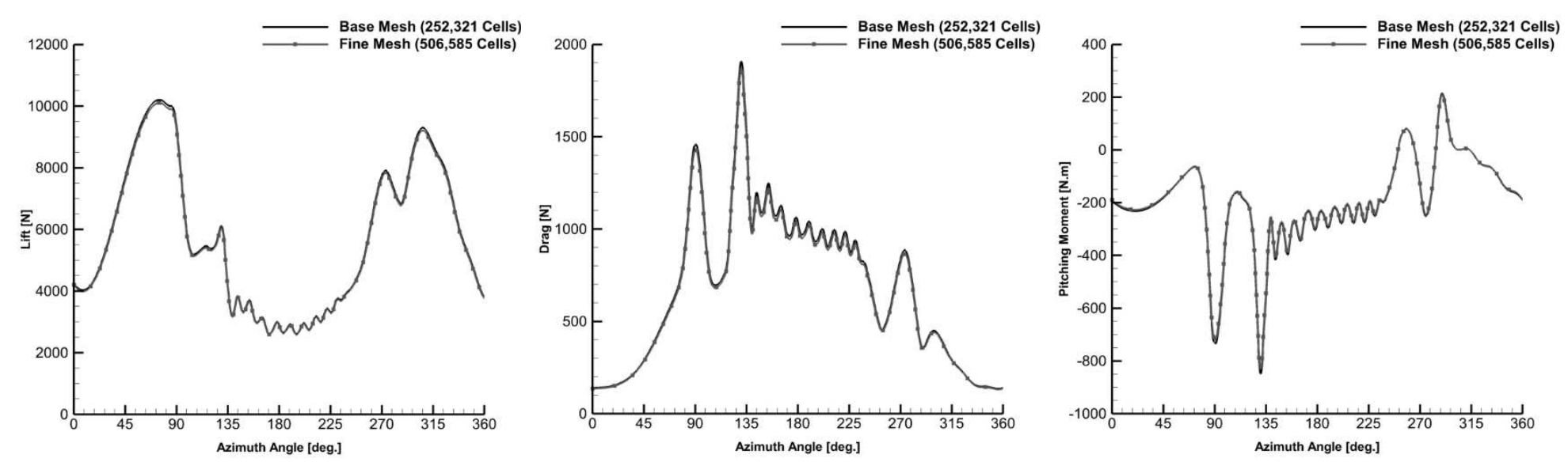

Figure 3.23 Results of the grid dependent study. SC1095, Frequency $=4.25 \mathrm{~Hz}, R e=$

$$
6.1 \times 10^{6}, M=0.537 \pm 0.205 \text { and } \alpha=10^{\circ} \pm 10^{\circ} .
$$

\subsubsection{Validation}

The validation process was divided into two parts. In the first one, a transonic steady-flow was compared to experiment to validate the ability of the current mesh to capture transonic flow on the advancing helicopter blades featuring a shockwave on the airfoil surface. In the second part, the methodology of simulating dynamic stall under fluctuating freestream conditions was compared to experiments which, however, did not featured transonic flow.

It is important to draw the attention to the fact that dynamic stall simulation under fluctuating freestream has never been validated before in the literature. And as such, this thesis will be the first one to provide such validation, presenting yet another novelty as well as supporting the reliability of the proposed simulation technique. 


\section{A. Transonic validation}

In this case a SC1095 airfoil was subjected to a transonic steady flow of Mach number 0.8 and Reynolds number of $5.65 \times 10^{6}$. These conditions correspond to the wind tunnel data of [104] for angles of attack of $2.1^{\circ}$ and $6.2^{\circ}$. The experiment in [104] was designed to test full scale rotorcraft airfoils at full scale Reynolds numbers and Mach numbers in the NASA Ames Eleven-Foot Transonic Wind Tunnel. Fig. 3.24 provides the pressure coefficient at the designated angles of attack. As illustrated in the figure, an excellent agreement was reached using the proposed mesh in this study. Hence, this mesh was considered to be suitable for simulating fluctuating freestream dynamic stall where shock-induced flow separation might occur.
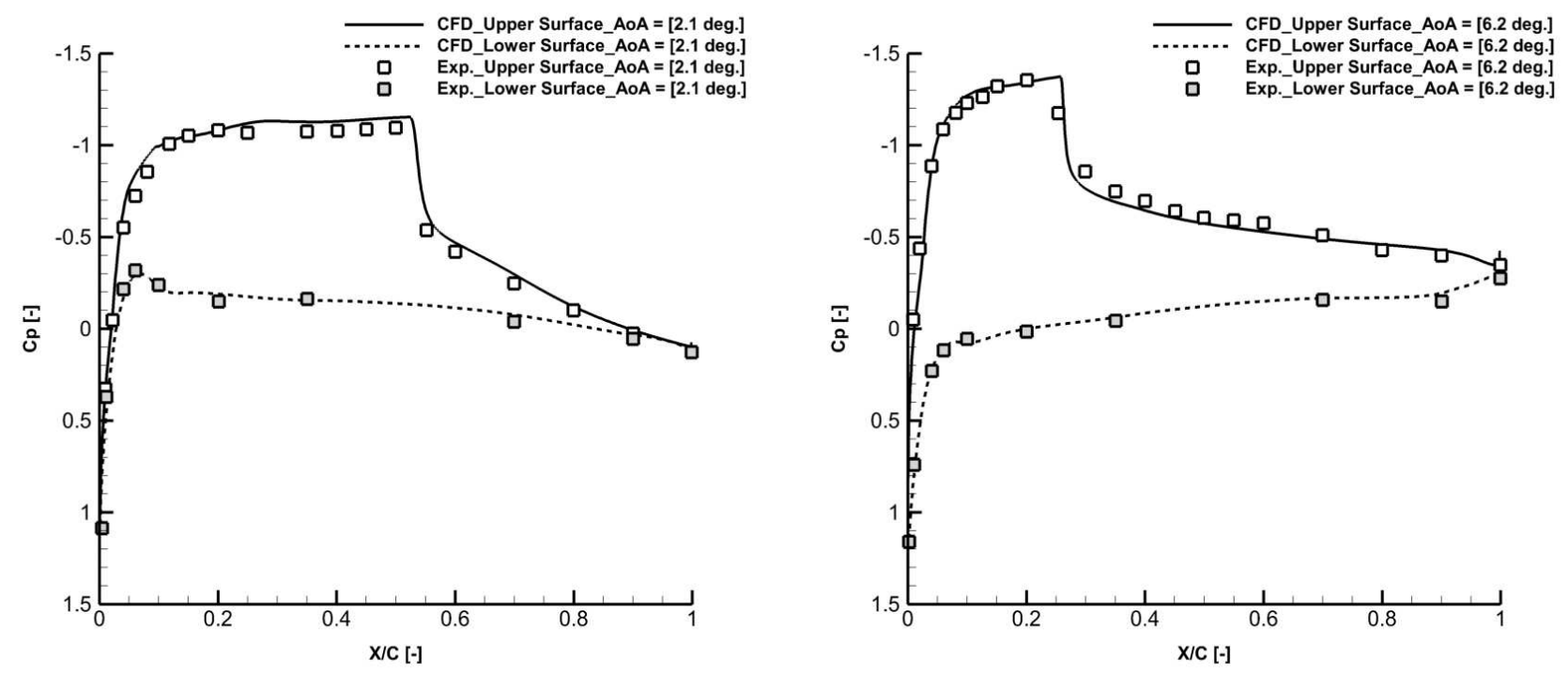

Figure 3.24 Transonic flow validation. SC1095 airfoil, $R e=6.65 \times 10^{6}, M=0.8$. 


\section{B. Fluctuating freestream validation}

The validation in this section was accomplished with the help of [77], [78], [105] and [106]. The experiment involves a SSC-A09 airfoil undergoing a dynamic stall in a fluctuating freestream environment, with the reduced frequency of 0.05 for the simultaneous pitching oscillation of $8.5^{\circ} \pm 13^{\circ}$ and Mach fluctuation of $0.4 \pm 0.08$. The airfoil's oscillation is given by Equation (3.7) while the Mach number fluctuation is approximated by Equation (3.8). Note that equation (3.8) is adopted from Ref. 76, where $\Delta \varphi$ represents the phase difference.

$$
\begin{aligned}
& \alpha(t)=\alpha_{o}-\Delta \alpha \cos (\Omega t) \\
& M(t)=M_{o}+\Delta M \cos (\Omega t-\Delta \varphi)
\end{aligned}
$$

Although this experiment has a very low Mach number amplitude (0.08) compared to that of the current case $(0.205)$, as well as it only focused on recording only lift and pitching moment (drag was omitted), it was decided to be used here for validation purposes because so far this is the only experiment that involves dynamic stall representative of helicopter forward flight as well as some moderate freestream fluctuation. Also, the frequency of pitch and Mach fluctuations is representative of helicopters, up to $17 \mathrm{~Hz}$ [106]. However, the experiment still has some considerable limitations, which can be summarized as: 
A. Pressure tabs limitation: The airfoil model in [106] was outfitted with 53 surface pressure taps that cover approximately $80 c \%$ of the airfoil from the leading edge, i.e. the rest of the $20 c \%$ of the airfoil was left without pressure taps due to geometrical limitation. Hence, the pressure at the trailing edge vicinity was estimated via a ghost tap in post-processing, which might lead to a possible post-processing error. Moreover, due to the insufficient resolution of the pressure taps near the leading edge [106], the accuracy of the results was significantly affected, especially when the leading-edge vortex was convecting downstream on the airfoil upper surface with the increasing angle of attack.

B. Test section aspect-ratio: The test section used in [106] had an aspect-ratio of 1. According to [106], at such low aspect-ratio the two-dimensionality of the flow was likely not perfect, which could lead to an artificially high velocity in the mid-span region because of the end wall boundary layers constricting the flow. Also, the low aspect-ratio was responsible for delaying the onset of dynamic stall and promoting an early dynamic reattachment, weakening the leading-edge vortex. As a result, the pitching moment peak was smaller than expected (lower strength) and the effective angles of attack were changed due to the downwash caused by the end walls of the test section.

C. Pitch oscillation assembly vibration: when the experiment of [106] was designed, the intended maximum angle of attack was $20^{\circ}$. However, with the unpowered pitching amplitude, and the significant vibration in the oscillation assembly, this made the measured amplitude angle of attack to be increasing with frequency. Therefore, when the pitching amplitude increased, the angular acceleration and the effective frequency at the onset of stall or reattachment were slightly higher than the design values. 
Figs. $3.25,3.26$ and 3.27 illustrates the results of the entire validation process. As one can see, fluctuation freestream simulation matches the experimental data quite well especially when one considers the limitations of the experiment above and the expected overshoots in the peak values of the hysteresis loops. The discrepancy in the downstroke phase of moment is very common in the literature even for constant freestream dynamic stall and is likely linked to the turbulence model used. Finally, note that in the experiment of [106] the following uncertainty levels occurred: for Mach number \pm 0.005 ; for Reynolds number \pm 5000 , for angle of attack $\pm 0.05^{\circ}$, for $C_{p}, \pm 0.05$, for $C_{l}, \pm 0.05$, for $C_{m}$, \pm 0.02 , and for $T_{\infty}, \pm 0.05 \mathrm{~K}$.

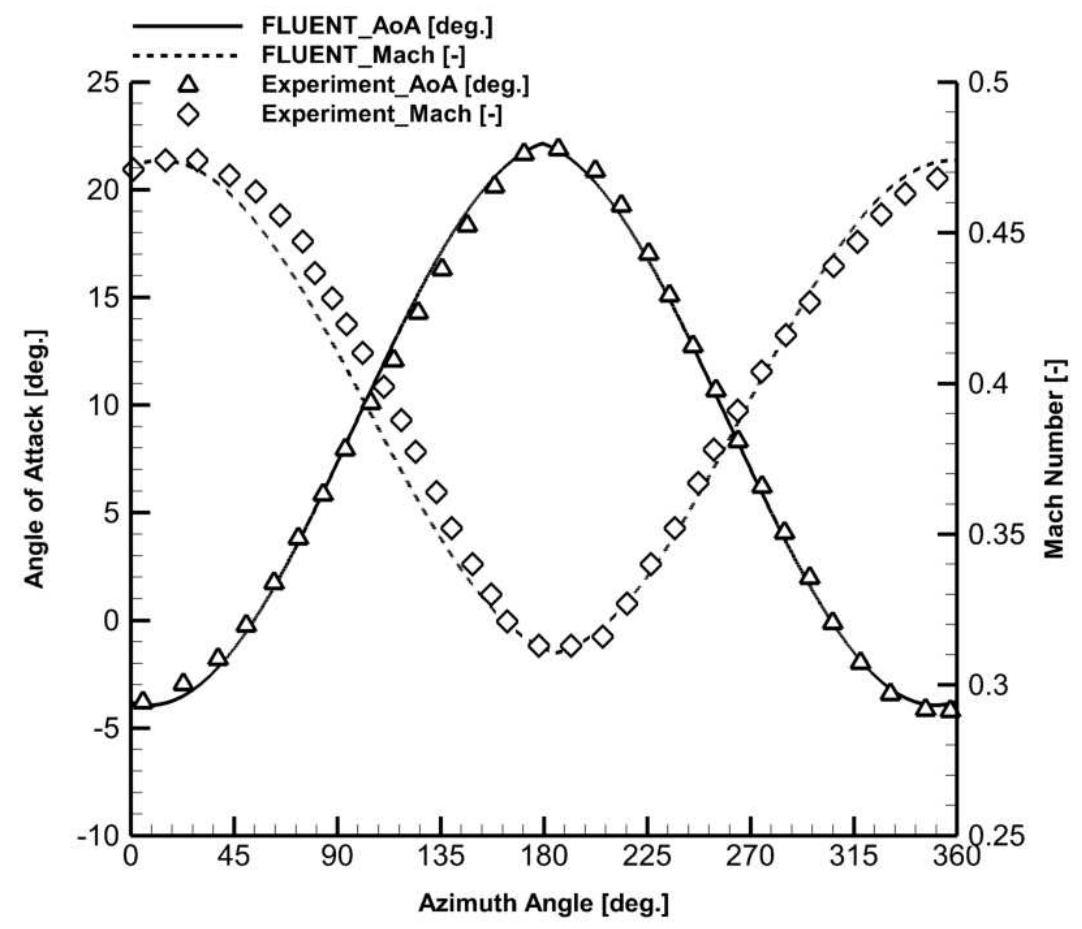

Figure 3.25 Validation of the fluctuating freestream conditions at the airfoil leading edge with the experiments of [106]. $R e=3 \times 10^{6}, \Delta \varphi=13.3^{\circ}, k=0.05, \alpha=8.5 \pm 13^{\circ}$ and $M=$

$$
0.4 \pm 0.08
$$



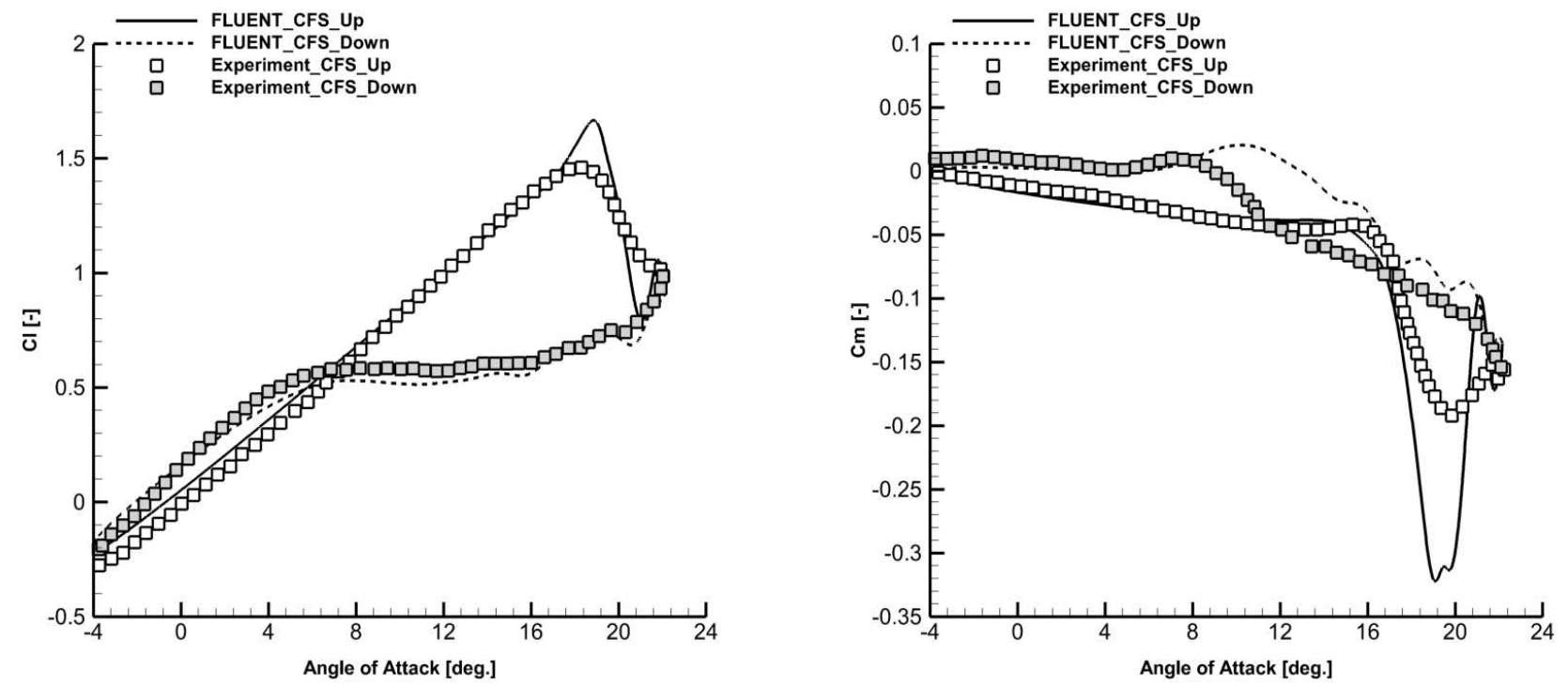

Figure 3.26 Constant freestream (CFS) dynamic stall validated with [106]. SSC-A09 airfoil, $\operatorname{Re}=3 \times 10^{6}, k=0.05, \alpha=8.5 \pm 13$ and $M=0.4$.
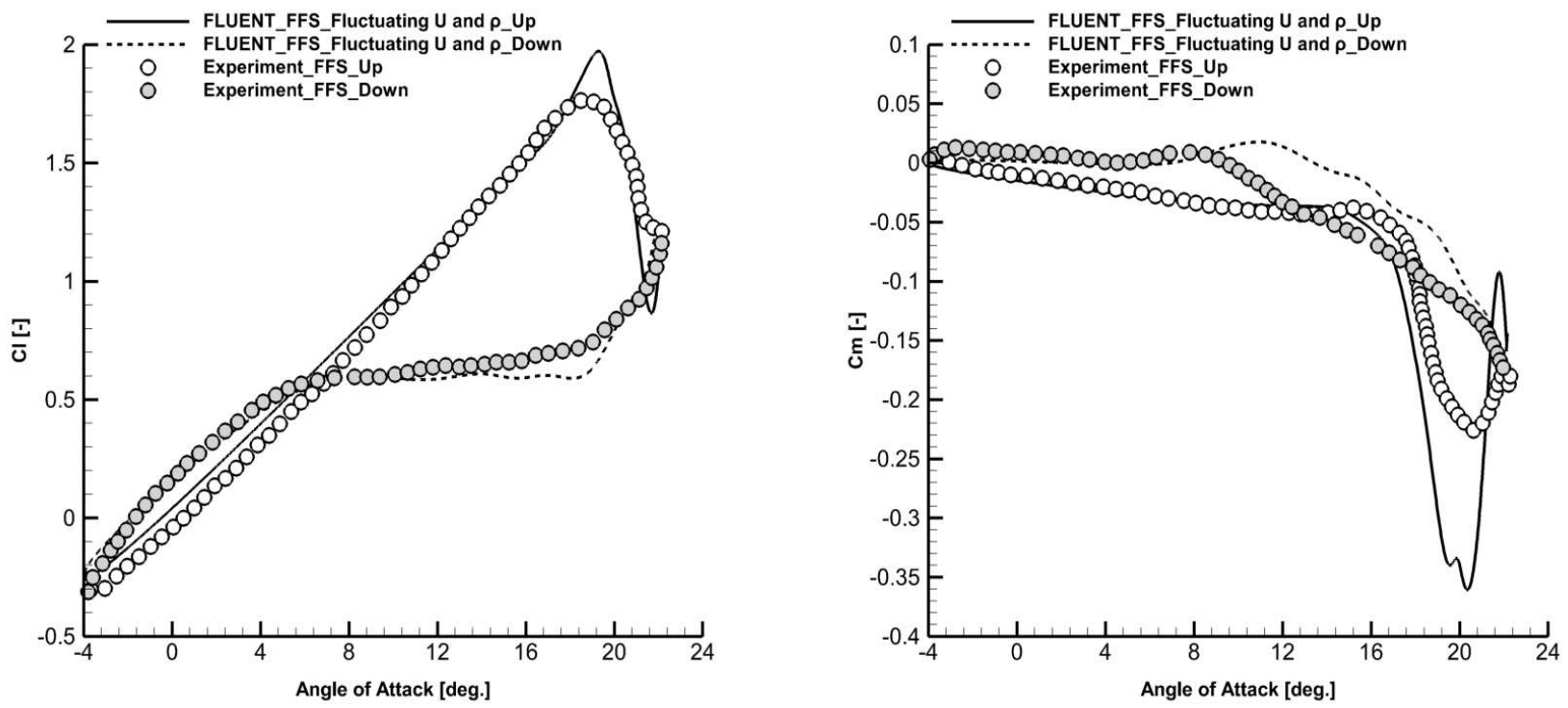

Figure 3.27 Fluctuating freestream (FFS) dynamic stall validated with [106]. SSC-A09 airfoil, $R e=3 \times 10^{6}, \Delta \varphi=13.3^{\circ}, k=0.05, \alpha=8.5 \pm 13$ and $M=0.4 \pm 0.08$. 


\section{Chapter 4}

\section{Results and Discussions}

\subsection{Chapter Overview}

As noted earlier, this thesis aims to investigate the effect of the proposed novel passive flow control technique and its variants on the performance of both rotary- and fixed-wings aircraft. The study will focus on two major flow regimes, transonic cruise of commercial jets as well as helicopters blade dynamic stall during forward flight (at constant and fluctuating freestreams). Accordingly, the results discussed in the chapter will follow the same sequential order of Chapter 3 to preserve the structure of the thesis.

The chapter begins with a list of the investigated passive flow control geometries. The list includes the stepped airfoils, termed as " $1{ }^{\text {st }}$ Generation," and the newly designed, surface-based trapped vortex generator geometries, termed as " 2 nd, $3^{\text {rd }}$, and $4^{\text {th }}$ Generations" in this work. 


\subsection{Passive Flow Control Geometries}

Recall that the novel passive flow control geometries were inspired by airfoils with Backward Facing Steps (BFS), i.e. stepped airfoils. Therefore, the stepped airfoils were also part of the research covered in this thesis. It is worth to mention that it will be a novelty to investigate the airfoils with BFS at high Reynolds numbers and Mach numbers. The discussion provides an understanding on how the presence of a step with the associated recirculation vortex affects the upstream flow (shockwave strength) and the downstream flow (flow reattachment and stall characteristics). The studied BFS configurations was chosen according to the experimental study of [22]. From the many configurations attempted, only the best three will be presented in this document.

Regarding the geometric specifications, the thesis will employ the same terminology as in [22] to identify the changes in the BFS configurations. Fig. 4.1 shows a stepped airfoil with the geometry specifications.

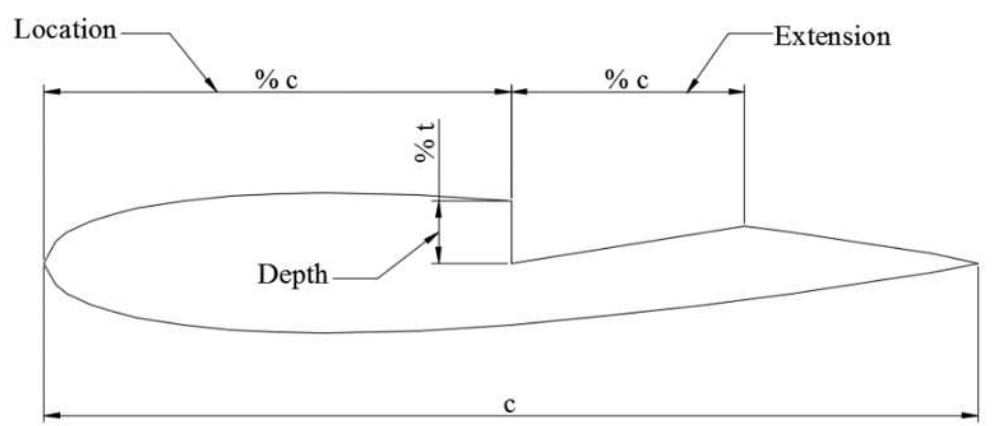

Figure 4.1 Schematic of an airfoil with the geometric specifications of the BFS [22]. 
The passive flow control is created by simply modifying one of the airfoil surfaces by implementing a geometrical alteration termed as "Surface-based Trapped Vortex Generator" or STVG. Note that a "trapped vortex generator" is any type of surface modification leading to the occurrence of a trapped vortex. This excludes the classical "backward facing step" but not the new geometries, as will be proposed in this work. The modified airfoils were developed by considering 32 different geometries (13 on the upper surface and 13 on the lower surface plus 6 mixed configurations) for generating trapped vortices. The geometries presented in this thesis were arrived to after meticulous partial-optimization (at the location of the STVG) studies by investigating their effects systematically. The optimization process was made by studying and modifying the pressure distribution on the surfaces of the airfoil until the final design was reached. This was made with the help of the FLUENT Adjoint Solver Technology [107]. The optimization goal was to find the best shape and location for the surface modification for which it would provide an optimal performance. In addition to the stepped airfoils, three groups of novels geometries were developed and investigated. The four groups based on their main features are: stepped airfoils, trailing-vortex airfoils, body-trapped vortex airfoils and leading-trailing vortex airfoils.

The main reason behind inventing such large number modifications was to provide a large variety of trapped vortex concepts, from which one can select the appropriate design for a specific application. Fig. 4.2 provides an illustration of the four passive flow control concepts generations (implemented on the surfaces of the airfoil), and Table 4.1 gives the geometric specifications. 
$1^{\text {st }}$ Generation: Backward Facing Step Modifications

$\mathrm{G}^{1} \mathrm{U} 1$
$\mathrm{G}^{1} \mathrm{U} 2$
$\mathrm{G}^{1} \mathrm{U} 3$

$3^{\text {rd }}$ Generation: Body-trapped Vortex Modifications
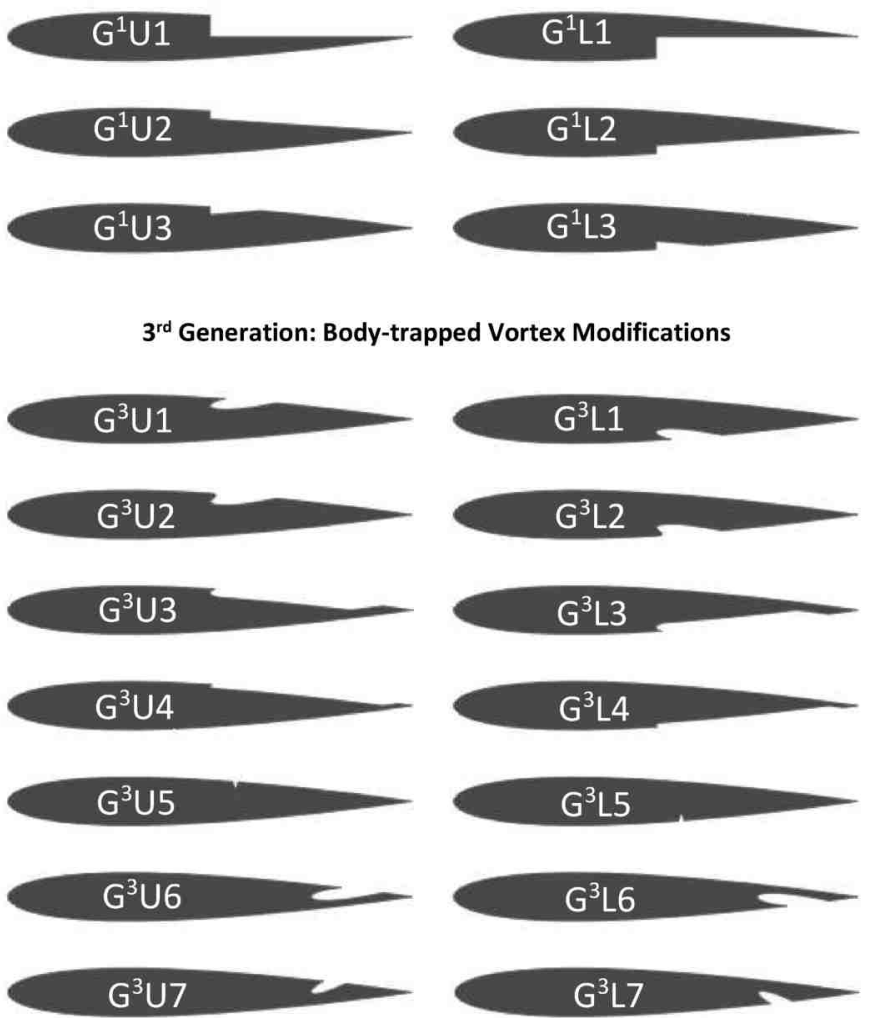

$2^{\text {nd }}$ Generation: Trailing-vortex Modifications
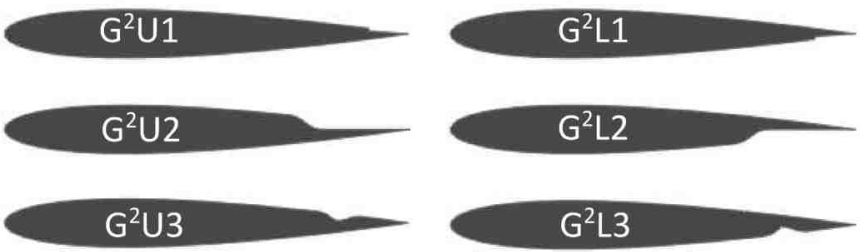

$4^{\text {th }}$ Generation: LE-TE Vortices Modifications

\section{$\mathrm{G}^{4} \mathrm{LTV} 1$}

$\mathrm{G}^{4} \mathrm{LTV} 2$

$\mathrm{G}^{4} \mathrm{LTV} 3$
$\mathrm{G}^{4}$ LTV1i

$\mathrm{G}^{4} \mathrm{LTV} 2 \mathrm{i}$

$\mathrm{G}^{4} \mathrm{LTV} 3 \mathrm{i}$

Figure 4.2 Airfoils with the passive geometries investigated in the study.

\subsection{Passive Flow Control for 2D Transonic Aerodynamic Improvement}

In this section baseline airfoils were selected to be a NACA 0012 and NASA $\mathrm{SC}(3)-0712$ (B) airfoils, because of the availability of transonic experimental data from NASA [88, 89]. It should be also noted that the baseline airfoils geometries were designed based on the coordinates reported in the experiment reports, i.e. they have thick and blunt trailing edges to match the conditions of the experiments as much as possible. 
Table 4.1 Geometric specifications of the studied passive flow control.

Upper Surface Modifications

$I^{\text {st }}$ Generation: Backward Facing Step Modifications

\begin{tabular}{|c|c|c|c|}
\hline Model & Location $\% c$ & Depth \% $t$ & Extent $\% c$ \\
\hline $\mathrm{G}^{1} \mathrm{U} 1$ & 50.00 & 50.00 & To the TE \\
\hline $\mathrm{G}^{1} \mathrm{U} 2$ & 50.00 & 19.00 & To the TE \\
\hline $\mathrm{G}^{1} \mathrm{U} 3$ & 50.00 & 19.00 & 12.50 \\
\hline
\end{tabular}

$2^{\text {nd }}$ Generation: Trailing-vortex Modifications

\begin{tabular}{|c|c|c|c|}
\hline Model & Location $\% c$ & Depth $\% t$ & Extent $\% c$ \\
\hline $\mathrm{G}^{2} \mathrm{U} 1$ & 90.00 & 25.00 & To the TE \\
\hline $\mathrm{G}^{2} \mathrm{U} 2$ & 75.00 & 50.00 & To the TE \\
\hline $\mathrm{G}^{2} \mathrm{U} 3$ & 82.00 & 45.26 & 10.54 \\
\hline
\end{tabular}

$3^{\text {rd }}$ Generation: Body-trapped vortex Modifications

\begin{tabular}{|c|c|c|c|}
\hline Model & Location $\% c$ & Depth \% $t$ & Extent $\% c$ \\
\hline $\mathrm{G}^{3} \mathrm{U} 1$ & 50.00 & 17.40 & 16.40 \\
\hline $\mathrm{G}^{3} \mathrm{U} 2$ & 50.00 & 18.00 & 16.40 \\
\hline $\mathrm{G}^{3} \mathrm{U} 3$ & 50.00 & 19.80 & 42.80 \\
\hline $\mathrm{G}^{3} \mathrm{U} 4$ & 50.00 & 9.20 & 46.50 \\
\hline $\mathrm{G}^{3} \mathrm{U} 5$ & 56.00 & 16.50 & 1.27 \\
\hline $\mathrm{G}^{3} \mathrm{U} 6$ & 75.00 & 50.00 & 17.80 \\
\hline $\mathrm{G}^{3} \mathrm{U} 7$ & 75.00 & 50.00 & 8.50 \\
\hline
\end{tabular}

Lower Surface Modifications

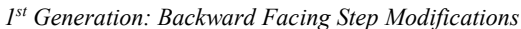

$\begin{array}{cccc}\text { Model } & \text { Location } \% c & \text { Depth \% } \boldsymbol{t} & \text { Extent \% } c \\ \mathrm{G}^{1} \mathrm{~L} 1 & 50.00 & 50.00 & \text { To the TE } \\ \mathrm{G}^{1} \mathrm{~L} 2 & 50.00 & 19.00 & \text { To the TE } \\ \mathrm{G}^{1} \mathrm{~L} 3 & 50.00 & 19.00 & 12.50\end{array}$

$2^{\text {nd }}$ Generation: Trailing-vortex Modifications

\begin{tabular}{|c|c|c|c|}
\hline Model & Location $\% c$ & Depth \% $t$ & Extent $\% c$ \\
\hline $\mathrm{G}^{2} \mathrm{~L} 1$ & 90.00 & 25.00 & To the TE \\
\hline $\mathrm{G}^{2} \mathrm{~L} 2$ & 75.00 & 50.00 & To the TE \\
\hline $\mathrm{G}^{2} \mathrm{~L} 3$ & 82.00 & 45.26 & 10.54 \\
\hline
\end{tabular}

$3^{\text {rd }}$ Generation: Body-trapped vortex Modifications

\begin{tabular}{|c|c|c|c|}
\hline Model & Location $\% c$ & Depth \% $t$ & Extent $\% c$ \\
\hline $\mathrm{G}^{3} \mathrm{~L} 1$ & 50.00 & 17.40 & 16.40 \\
\hline $\mathrm{G}^{3} \mathrm{~L} 2$ & 50.00 & 18.00 & 16.40 \\
\hline $\mathrm{G}^{3} \mathrm{~L} 3$ & 50.00 & 19.80 & 42.80 \\
\hline $\mathrm{G}^{3} \mathrm{~L} 4$ & 50.00 & 9.20 & 46.50 \\
\hline $\mathrm{G}^{3} \mathrm{~L} 5$ & 56.00 & 16.50 & 1.27 \\
\hline $\mathrm{G}^{3} \mathrm{~L} 6$ & 75.00 & 50.00 & 17.80 \\
\hline $\mathrm{G}^{3} \mathrm{~L} 7$ & 75.00 & 50.00 & 8.50 \\
\hline
\end{tabular}

Leading and Trailing Edges Modifications

$4^{\text {th }}$ Generation: LE-TE Vortex Modifications

$\begin{array}{cc}\text { Model } & \text { Location \% } c \\ \mathrm{G}^{4} \mathrm{LTV1} & 00.00 \mathrm{LE} \& 99.68 \mathrm{TE} \\ \mathrm{G}^{4} \mathrm{LTV} 2 & 00.00 \mathrm{LE} \& 95.37 \mathrm{TE} \\ \mathrm{G}^{4} \mathrm{LTV3} & 00.00 \mathrm{LE} \& 87.21 \mathrm{TE}\end{array}$

$\mathrm{G}^{4} \mathrm{LTV} 3$

$4^{\text {th }}$ Generation: Inversed LE-TE Vortex Modifications

Model

$\mathrm{G}^{4}$ LTV1i

$\mathrm{G}^{4} \mathrm{LTV} 2 \mathrm{i}$

$\mathrm{G}^{4} \mathrm{LTV} 3 \mathrm{i}$
Location \% $c$ $00.00 \mathrm{LE} \& 99.68 \mathrm{TE}$ $00.00 \mathrm{LE} \& 95.37 \mathrm{TE}$ 00.00 LE \& 87.21 TE
Depth \% $t$ $50.00 \mathrm{LE} \& 00.00 \mathrm{TE}$ $50.00 \mathrm{LE} \& 50.00 \mathrm{TE}$ $50.00 \mathrm{LE} \& 50.00 \mathrm{TE}$
Extent \% $c$ $50.00 \mathrm{LE} \& 00.00 \mathrm{TE}$ $50.00 \mathrm{LE} \& 50.00 \mathrm{TE}$ $50.00 \mathrm{LE} \& 50.00 \mathrm{TE}$

1.67 LE \& $0.32 \mathrm{TE}$ 1.67 LE \& 4.63 TE $1.67 \mathrm{LE} \& 12.79 \mathrm{TE}$ 
In the current section, only three from the four modification groups above will be discussed in detail on a NACA 0012 airfoil, followed by the demonstration of the modifications on a NASA SC(3)-0712(B) supercritical airfoil. Fig. 4.3 illustrates the selected airfoils with their corresponding group terminology.
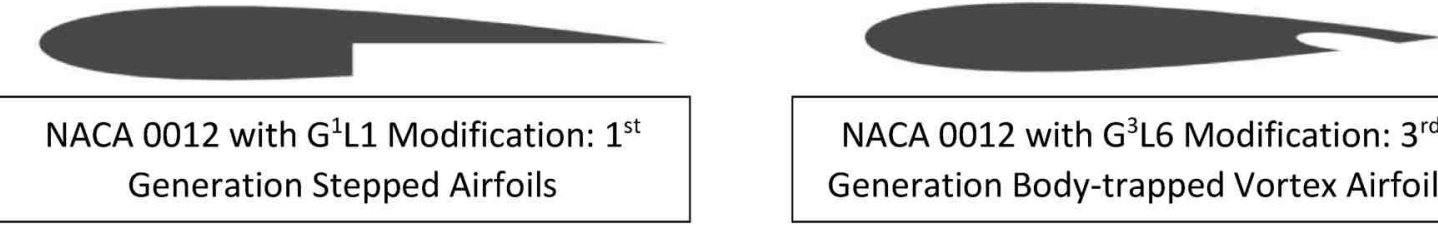

NACA 0012 with $\mathrm{G}^{3}$ L6 Modification: $3^{\text {rd }}$ Generation Body-trapped Vortex Airfoils

NACA 0012 with $\mathrm{G}^{4}$ LTV1 Modification: $4^{\text {th }}$

Generation Leading-Trailing Vortices Airfoils

NASA SC(3)-0712(B) with $\mathrm{G}^{2} U 3$ Modification: $2^{\text {nd }}$ Generation Trailing-Vortex Airfoils
NASA SC(3)-0712(B) with $G^{4} L T V 1$ i Modification: $4^{\text {th }}$ Generation Leading-Trailing Vortices Airfoils

Figure 4.3 Geometry modifications on a NACA 0012 and NASA SC(3)-0712(B) airfoils.

\subsubsection{NACA 0012 Airfoil (Test Case 1)}

CFD simulations of 2D transonic flow for all 32 airfoils (NACA 0012 with modifications) were completed with the numerical parameters described in Chapter 3. The summary of the simulation results is shown in Table 4.2. In all cases, simulations were conducted between $A o A$ of $0^{\circ}$ to $5^{\circ}$. The performance of an airfoil was judged based on the average $A o A\left(2.5^{\circ}\right)$ because it represents a typical cruise angle of attack of a commercial jet or the advancing blade angle of attack of a helicopter in forward flight. In both scenarios, shockwave-induced separation is expected to occur. 
Recall that the research of this work aims to design such passive flow control concept, which is an alternative to the conventional stepped airfoils and which does not deteriorate the desired aerodynamic characteristics of the original airfoil, while offering significant improvements in the lift-to-drag ratio or by decreasing wave drag.

As can be seen from Table 4.2 , the proposed novel techniques (i.e. $2^{\text {nd }}, 3^{\text {rd }}$ and $4^{\text {th }}$ generation airfoils) perform remarkably well in comparison to the conventional stepped airfoil (termed as $1^{\text {st }}$ generation: backward facing step modifications in Table 4.2). For most modifications, the shockwave-induced separation was successfully decreased, which leads to a noteworthy drag reduction. The highest drag reduction was achieved when using the modification on the upper surface of the airfoil. Yet, lift was significantly increased when using the proposed new concepts on the lower surface of the airfoil.

For all the newly proposed concepts $\left(2^{\text {nd }}, 3^{\text {rd }}\right.$ and $4^{\text {th }}$ generations $)$, as much as $44 \%$ increase in the lift-to-drag ratio was achieved regardless of whether the geometry modification was implemented on the upper or lower surface of the airfoil.

On the other hand, the conventional stepped airfoils (i.e. the $1^{\text {st }}$ generation), with a surface modification on the upper surface failed to increase the lift-to-drag ratio, indicating that what worked at low speeds, (gliders and RC airplanes [17-25]), might not be suitable for high Mach number and high Reynolds number transonic flows. This was the main reason behind seeking alternative designs to the stepped airfoils.

The search for the reasons for these phenomena was conducted through the detailed analysis of the flow field for all airfoils. Due to space constraints, only a selection of these detailed results (not necessarily the best) is provided in the following sections for 3 modified NACA 0012 airfoils (Fig. 4.3 and indicated by gray arrow in Table 4.2), two with lower surface and one with a leading-edge modification. 
Table 4.2 Results summary of the modified NACA 0012 airfoil.

Upper Surface Modifications

$I^{\text {st }}$ Generation: Stepped Airfoils

\begin{tabular}{|c|c|c|c|c|}
\hline \multirow[b]{2}{*}{ Model } & \multicolumn{4}{|c|}{$A o A=2.5 \mathrm{deg}$} \\
\hline & $\Delta C_{d}[-] \%$ & $\Delta C_{l}[-] \%$ & $\Delta C_{m}[-] \%$ & $\Delta C_{l} / C_{d}[-] \%$ \\
\hline $\mathrm{G}^{1} \mathrm{U} 1$ & 51.0 & -32.9 & -74.3 & -54.6 \\
\hline $\mathrm{G}^{1} \mathrm{U} 2$ & 18.9 & -23.3 & -78.0 & -34.0 \\
\hline $\mathrm{G}^{1} \mathrm{U} 3$ & 9.3 & 2.8 & -36.4 & -3.8 \\
\hline
\end{tabular}

$2^{\text {nd }}$ Generation: Trailing-vortex Airfoils

\begin{tabular}{|c|c|c|c|c|}
\hline \multirow[b]{2}{*}{ Model } & \multicolumn{4}{|c|}{$A o A=2.5 \mathrm{deg}$} \\
\hline & $\Delta C_{d}[-] \%$ & $\Delta C_{l}[-] \%$ & $\Delta C_{m}[-] \%$ & $\Delta C_{l} / C_{d}[-] \%$ \\
\hline $\mathrm{G}^{2} \mathrm{U} 1$ & -15.1 & 9.4 & -47.1 & 31.9 \\
\hline $\mathrm{G}^{2} \mathrm{U} 2$ & -13.3 & 1.2 & -64.4 & 19.5 \\
\hline $\mathrm{G}^{2} \mathrm{U} 3$ & -13.7 & 8.2 & -50.2 & 28.1 \\
\hline
\end{tabular}

$3^{\text {rd }}$ Generation: Body-trapped vortex Airfoils

\begin{tabular}{|c|c|c|c|c|}
\hline Model & \multicolumn{4}{|c|}{$A o A=2.5 \mathrm{deg}}$. \\
\hline $\mathrm{G}^{3} \mathrm{U} 1$ & -2.2 & 4.3 & -45.5 & 9.1 \\
\hline $\mathrm{G}^{3} \mathrm{U} 2$ & -1.2 & 3.4 & -48.8 & 7.1 \\
\hline $\mathrm{G}^{3} \mathrm{U} 4$ & -20.1 & -5.6 & -76.7 & 20.9 \\
\hline $\mathrm{G}^{3} \mathrm{U} 5$ & -12.0 & 13.7 & -29.1 & 32.1 \\
\hline $\mathrm{G}^{3} \mathrm{U} 6$ & -10.6 & 4.2 & -51.7 & 19.1 \\
\hline
\end{tabular}

Lower Surface Modifications

$1^{\text {st }}$ Generation: Stepped Airfoils

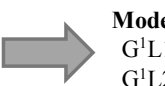

$\mathrm{G}^{1} \mathrm{~L} 1$

$G^{1} L 2$

\begin{tabular}{cccc}
\multicolumn{5}{c}{$\boldsymbol{A o \boldsymbol { A } = \mathbf { 2 . 5 } \mathrm { deg } .}$} \\
\hline $\boldsymbol{\Delta} \boldsymbol{C}_{\boldsymbol{d}[-]} \%$ & $\boldsymbol{\Delta} \boldsymbol{C}_{\boldsymbol{l}}[-] \%$ & $\boldsymbol{\Delta} \boldsymbol{C}_{\boldsymbol{m}}[-] \%$ & $\boldsymbol{\Delta} \boldsymbol{C}_{\boldsymbol{l}} / \boldsymbol{C}_{\boldsymbol{d}}[-] \%$ \\
84.6 & 65.8 & 58.2 & -12.4 \\
40.8 & 54.7 & 62.3 & 12.4 \\
17.1 & 26.3 & 66.4 & 10.4
\end{tabular}

$2^{\text {nd }}$ Generation: Trailing-vortex Airfoils

\begin{tabular}{|c|c|c|c|c|}
\hline \multirow[b]{2}{*}{ Model } & \multicolumn{4}{|c|}{$A o A=2.5 \mathrm{deg}$} \\
\hline & $\Delta C_{d}[-] \%$ & $\Delta C_{l}[-] \%$ & $\Delta C_{m}[-] \%$ & $\Delta C_{l} / C_{d}[-] \%$ \\
\hline $\mathrm{G}^{2} \mathrm{~L} 1$ & -10.9 & 18.9 & -6.4 & 36.6 \\
\hline $\mathrm{G}^{2} \mathrm{~L} 2$ & -2.8 & 28.8 & 77.3 & 35.5 \\
\hline $\mathrm{G}^{2} \mathrm{~L} 3$ & -7.8 & 20.8 & 34.1 & 34.0 \\
\hline
\end{tabular}

$3^{\text {rd }}$ Generation: Body-trapped vortex Airfoils

\begin{tabular}{|c|c|c|c|c|}
\hline \multirow[b]{2}{*}{ Model } & \multicolumn{4}{|c|}{$A o A=2.5 \mathrm{deg}}$. \\
\hline & $\Delta C_{d}[-] \%$ & $\Delta C_{l}[-] \%$ & $\Delta C_{m}[-] \%$ & $\Delta C_{l} / C_{d}[-] \%$ \\
\hline $\mathrm{G}^{3} \mathrm{~L} 1$ & 3.8 & 21.1 & 5.2 & 19.3 \\
\hline $\mathrm{G}^{3} \mathrm{~L} 2$ & 4.2 & 21.0 & 1.9 & 18.7 \\
\hline $\mathrm{G}^{3} \mathrm{~L} 3$ & -0.4 & 39.1 & 47.0 & 42.9 \\
\hline $\mathrm{G}^{3} \mathrm{~L} 4$ & -0.9 & 39.7 & 44.5 & 44.2 \\
\hline $\mathrm{G}^{3} \mathrm{~L} 5$ & -11.5 & 14.5 & -28.8 & 32.4 \\
\hline $\mathrm{G}^{3} \mathrm{~L} 6$ & 10.2 & 28.0 & -36.7 & 14.4 \\
\hline $\mathrm{G}^{3} \mathrm{~L} 7$ & -7.8 & 18.5 & -10.8 & 31.5 \\
\hline
\end{tabular}

Leading and Trailing Edges Modifications

$4^{\text {th }}$ Generation: LE-TE Vortices Airfoils

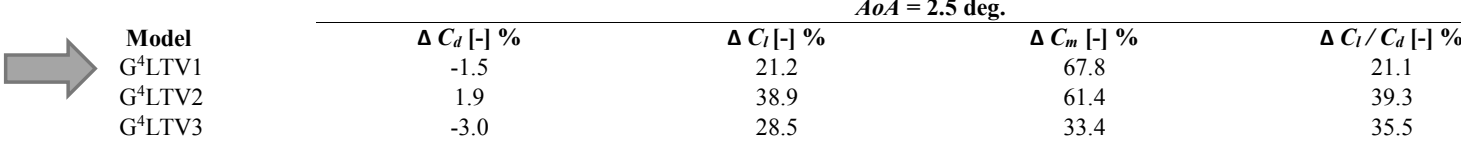

$4^{\text {th }}$ Generation: LE-TE Vortices Airfoils (Inverted)

\begin{tabular}{|c|c|c|c|c|}
\hline Model & \multicolumn{4}{|c|}{$A o A=2.5 \mathrm{deg}$} \\
\hline $\mathrm{G}^{4} \mathrm{LTV} 2 \mathrm{i}$ & -18.4 & -4.0 & -75.8 & 20.2 \\
\hline
\end{tabular}




\subsubsection{NACA 0012 with $\mathrm{G}^{1}$ L1 Modification}

The baseline airfoil has a BFS on the lower surface of the airfoil, located $0.5 c$ from the leading edge and a step depth of $0.5 t$, extended to the trailing edge, as shown in Fig. 4.3.

Fig. 4.4 provides the aerodynamic loads of the $\mathrm{G}^{1} \mathrm{~L} 1$ airfoil compared to the baseline airfoil. Locating the step at the lower surface produces higher lift compared to the baseline airfoil. Note that for the same lift, the $\mathrm{G}^{1} \mathrm{~L} 1$ airfoil needs to be oriented at only half of the angle of attack of the baseline airfoil.

Therefore, this airfoil outperforms the baseline airfoil at lower angles of attack, as illustrated in the lift-to-drag ratio diagram on Fig. 4.4. However, it also provides a larger negative pitching moment for the full range of the angle of attack compared to the baseline airfoil. It shall also be noted that the $\mathrm{G}^{1} \mathrm{~L} 1$ airfoil increases the strength of the shockwave, as shown in the $C_{P}$ plots in Fig. 4.5 as well as on the Mach number contours in Fig. 4.6, which leads to increased lift and drag. Fig. 4.7 depicts the $x$-component of the skin friction coefficient at 0.66 lift of the baseline airfoil, indicating the occurrence of a separation earlier in the baseline airfoil compared to that in the modified airfoil. Moreover, the separation of the modified airfoil followed by a weak re-attachment instead of the complete flow separation on the upper surface of the baseline airfoil.

The reason for the dramatic lift increase is likely due to the increase in the pressure inside the step due to the vortex on the lower surface as shown in Fig. 4.5 (the pressure inside the step increases at higher angles of attack). 

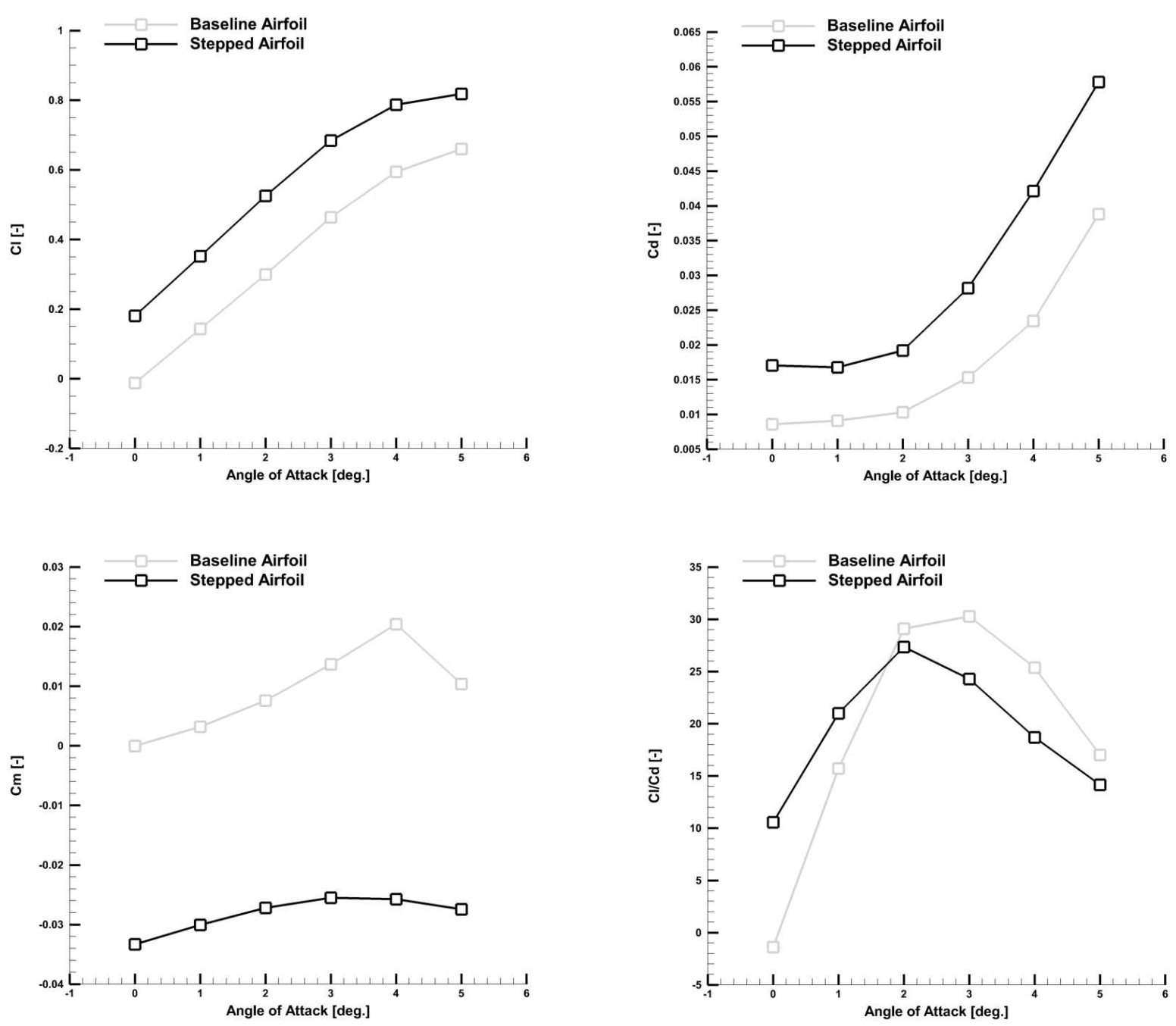

Figure 4.4 Aerodynamic loads of $\mathrm{G}^{1} \mathrm{~L} 1$ airfoil, $M=0.7$ and $R e=9 \times 10^{6}$.

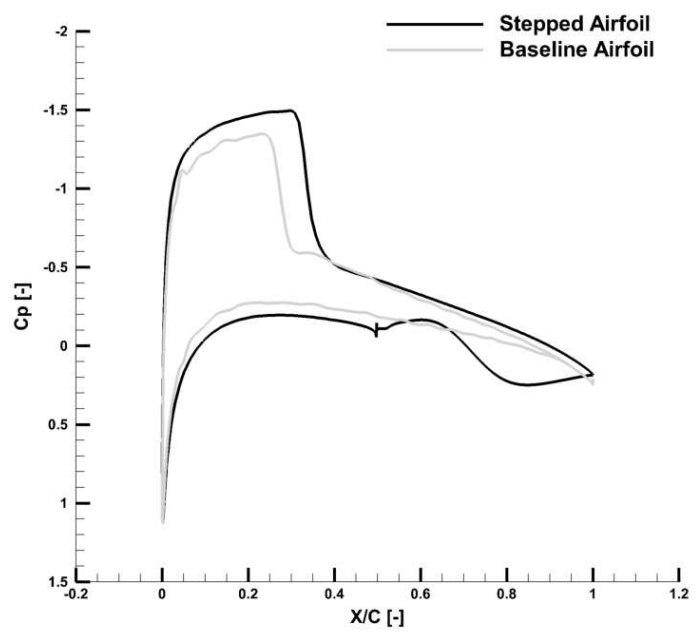

Figure 4.5 Pressure coefficients of $\mathrm{G}^{1} \mathrm{~L} 1$ airfoil, $M=0.7$ and $R e=9 \times 10^{6}, A o A=2.5^{\circ}$. 

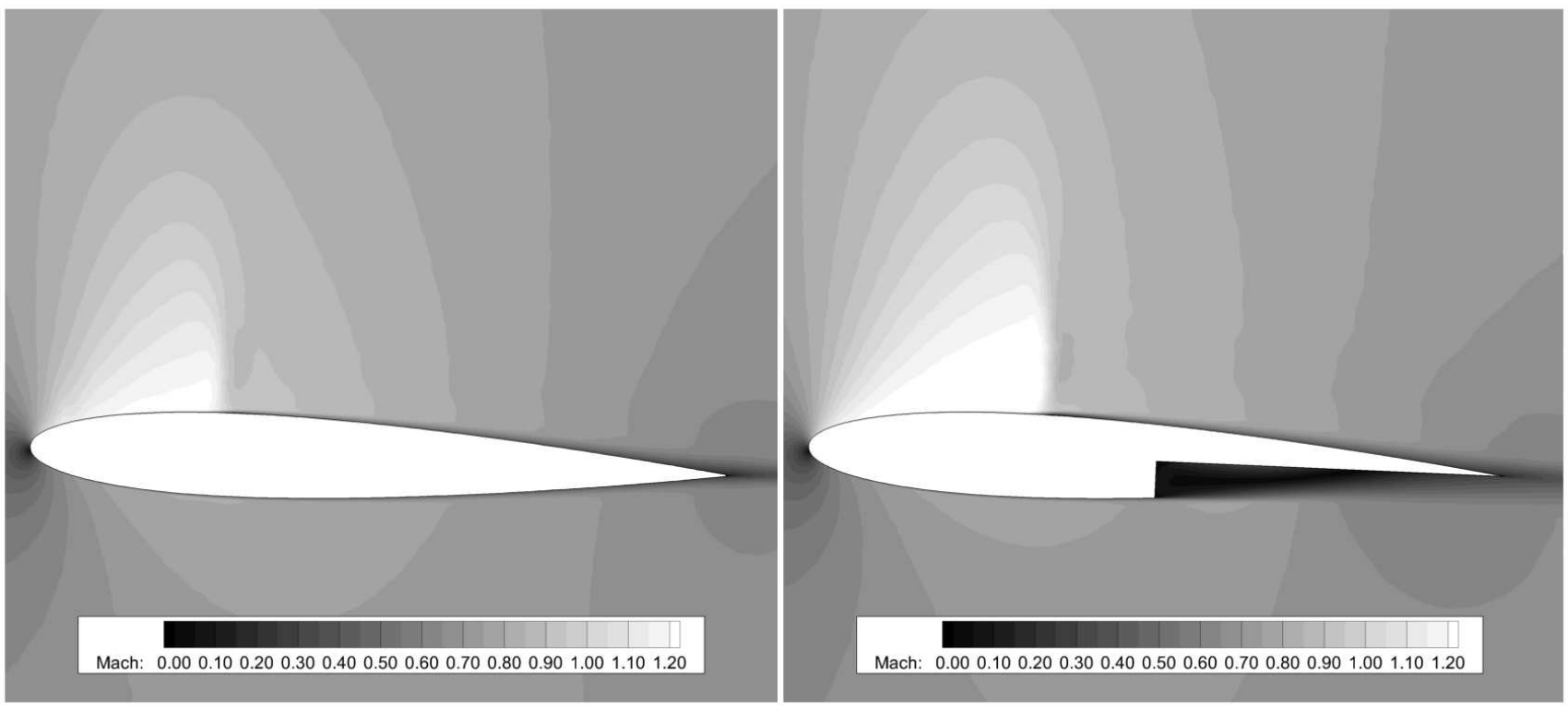

Figure 4.6 Mach number contours, baseline airfoil (left), $\mathrm{G}^{1} \mathrm{~L} 1$ airfoil (right), $M=0.7$ and $R e=$ $9 \times 10^{6}, A o A=2.5^{\circ}$.
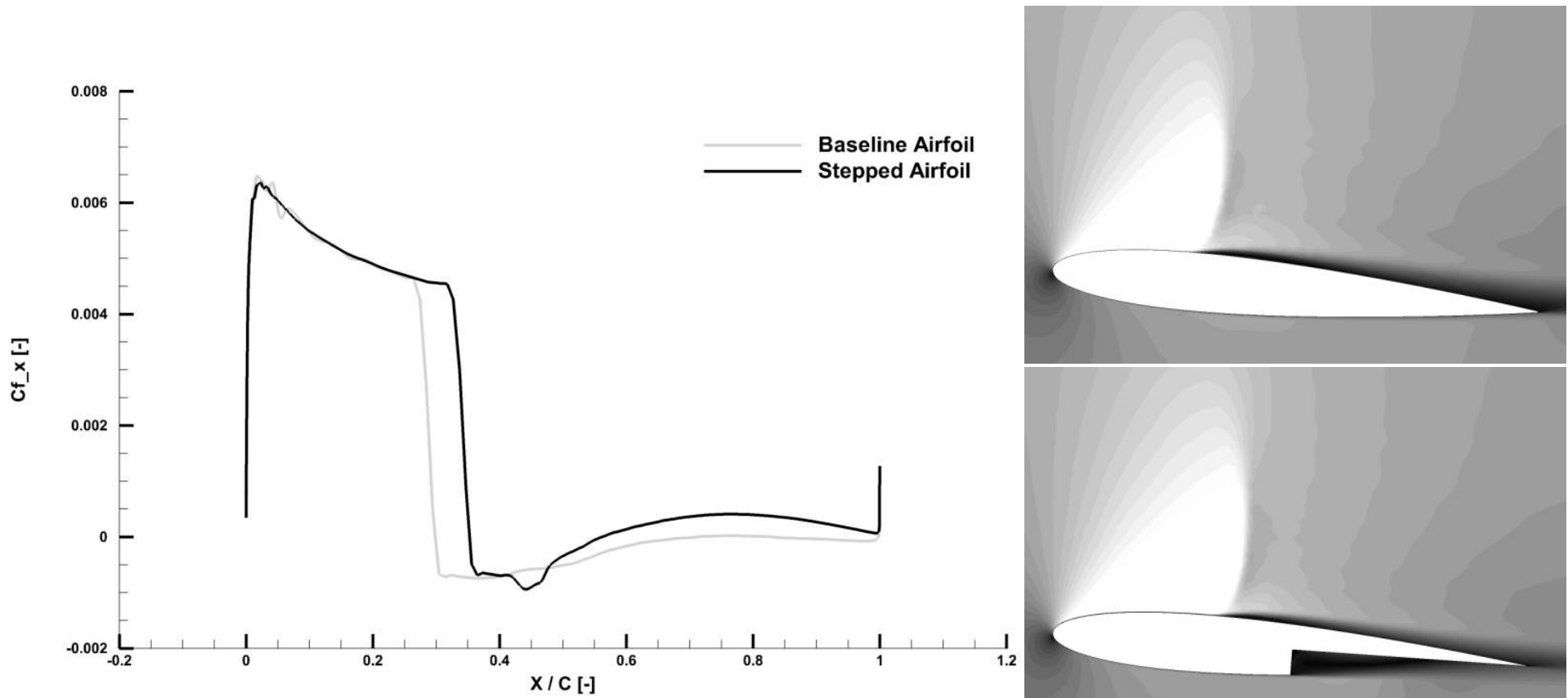

Figure 4.7 Skin friction coefficient (x-component) for the baseline airfoil and the airfoil with $\mathrm{G}^{1} \mathrm{~L} 1$ modification, upper surface, at $M=0.7$ and $R e=9 \times 10^{6}, C_{l}=0.66$. 


\subsubsection{NACA 0012 with $\mathrm{G}^{3}$ L6 Modification}

From Table 4.2 it is clear that placing the vortex on the airfoil's lower surface leads to an increase in the lift coefficient, and sometimes an increase of the drag coefficient as well due to a stronger shockwave. The $G^{3} L 6$ modification was designed with the aim to reduce the drag coefficient for a case when the vortex is placed on the upper or lower surface.

Fig. 4.3 illustrates the $\mathrm{G}^{3} \mathrm{~L} 6$ modification on the baseline airfoil, which literally "traps" the vortex inside a curved cavity towards the trailing edge of the lower surface of the airfoil. This was designed with the specific aim of preserving the vortex structure, i.e. without transforming the vortex into a random turbulent flow as seen in the case of the stepped airfoils. The modification is located on the lower surface, at $0.75 c$ from the airfoil's leading edge, with a cavity depth and length of $0.5 t$ and $0.178 c$, respectively.

The integrated aerodynamic characteristics are shown in Fig. 4.8. As can be seen, the main objective of the design was successfully achieved: the modified airfoil basically preserves or reduce the drag of the baseline airfoil while changing the lift and pitching moment only. Moreover, when compared to the baseline airfoil, the $G^{3} L 6$ airfoil shows major improvements in the lift-to-drag ratio, especially at lower angles of attacks. The shockwave has become slightly stronger, as illustrated by the $C_{P}$ plots on Fig. 4.9 which increase lift as shown in Table 4.2 and Fig. 4.10.

Although the cavity conceals the generated vortex, this did not prevent the vortex ability to help the upper surface boundary layer to re-attach. The outer edge of the vortex acted as if it was part of the airfoil skin and it was not causing any turbulence to the flow on the lower surface (Fig. 4.11). Also, in Fig. 4.11, it is worth to draw the attention to a 
minor separation bubble compared to that of the baseline airfoil that occurred earlier upstream.
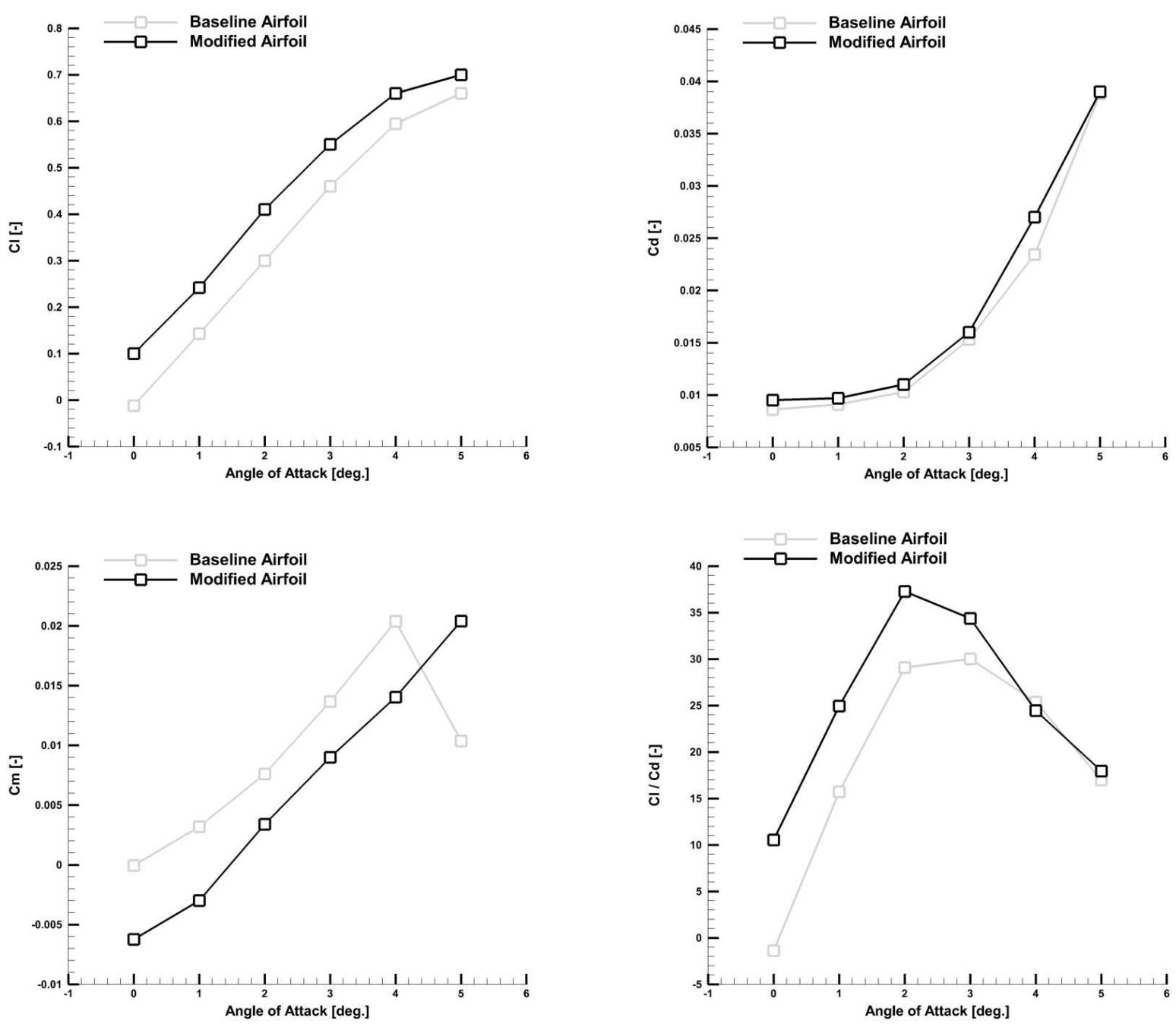

Figure 4.8 Aerodynamic loads of $\mathrm{G}^{3} \mathrm{~L} 6$ airfoil, $M=0.7$ and $R e=9 \times 10^{6}$. 


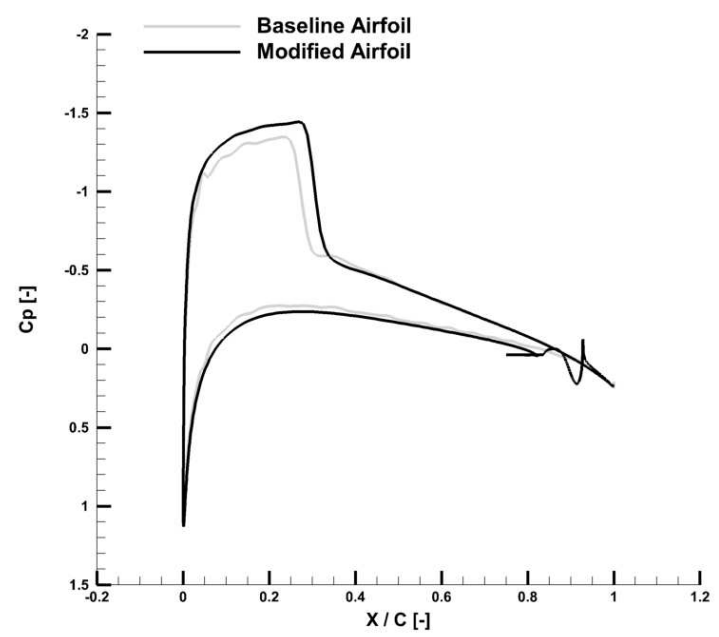

Figure 4.9 Pressure coefficients of $\mathrm{G}^{3} \mathrm{~L} 6$ airfoil, $M=0.7$ and $R e=9 \times 10^{6}, A o A=2.5^{\circ}$.
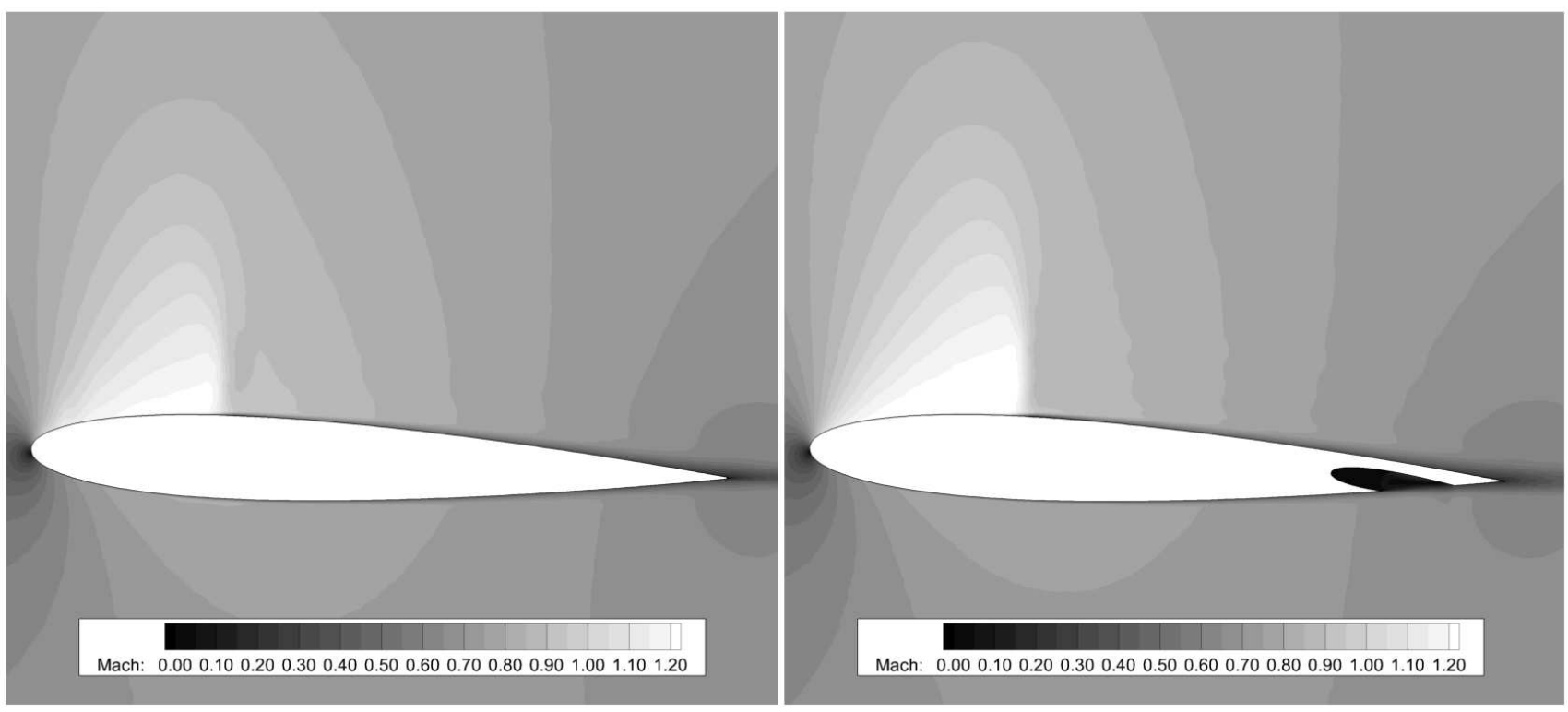

Figure 4.10 Mach number contours, baseline airfoil (left), $\mathrm{G}^{3} \mathrm{~L} 6$ airfoil (right), $M=0.7$

$$
\text { and } \operatorname{Re}=9 \times 10^{6}, A o A=2.5^{\circ} \text {. }
$$



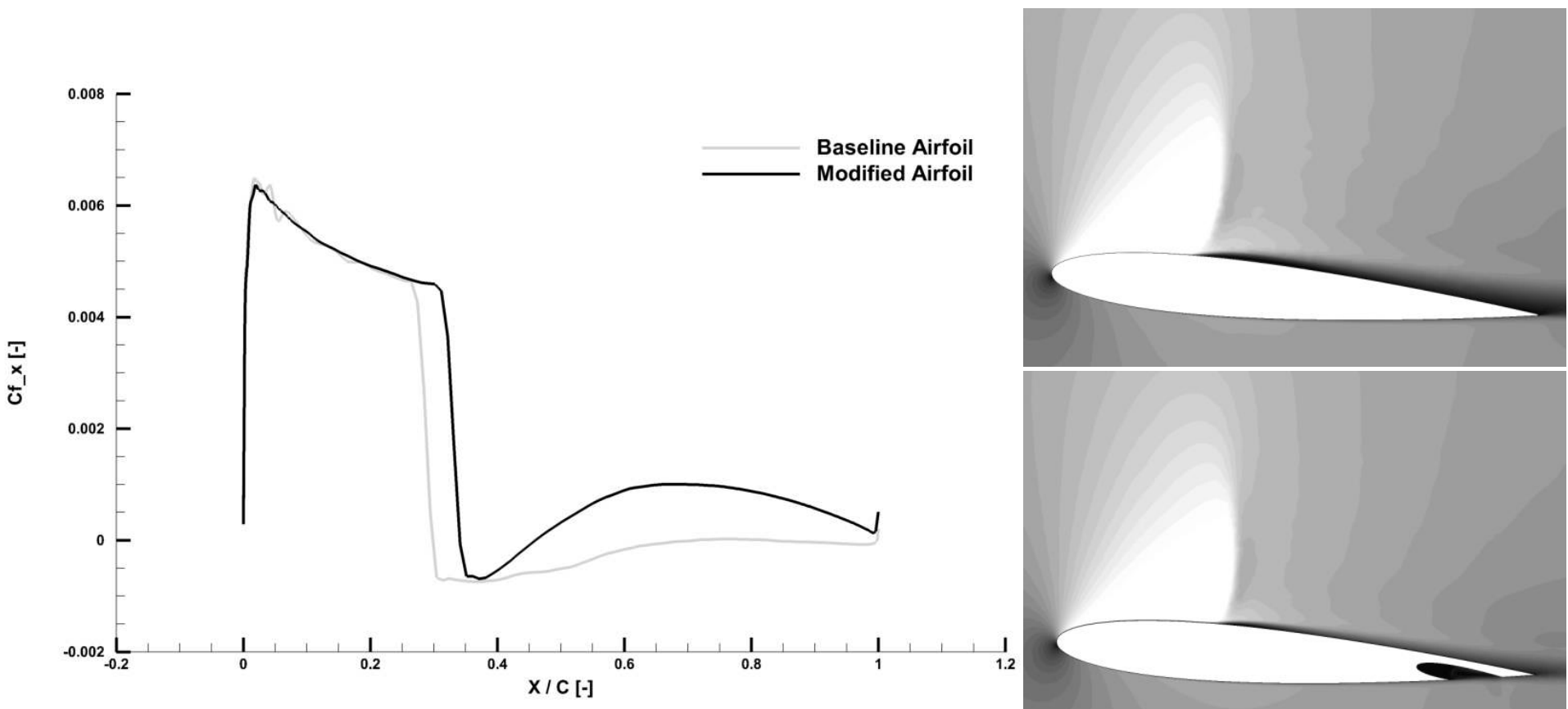

Figure 4.11 Skin friction coefficient (x-component) for the baseline airfoil and the airfoil with $\mathrm{G}^{3} \mathrm{~L} 6$ modification, upper surface, at $M=0.7$ and $R e=9 \times 10^{6}, C_{l}=0.66$.

\subsubsection{NACA 0012 with $G^{4} L T V 1$ Modification}

The $\mathrm{G}^{4} \mathrm{LTV} 1$ airfoil is the first of the fourth-generation "mixed leading-trailing edges modifications" designs, which aims to modify the shape of the leading and trailing edges of the baseline airfoil. The current modification includes only a curved face-cavity on the leading edge. The designs of the fourth-generation geometry modifications were concluded after careful partial optimization studies, and they are the first to introduce a "cavity technique" on the airfoil leading edge. Fig. 4.3 illustrates the G ${ }^{4}$ LTV1 modification on the baseline airfoil.

One of the most important aspects of this design is the small "nose vortex" that this airfoil's leading-edge cavity generates. The intended vortex is barely visible at lower 
angles of attack but grows larger at higher angles of attack, as seen in Fig. 4.12 for $A o A$ of $5^{\circ}$

It is surprising to see that with a simple modification of the airfoil leading edge shape, the aerodynamic characteristics of the airfoil can be improved significantly, as shown in Fig. 4.13. The lift increased right from angle of attack $0^{\circ}$, yet: at virtually no penalty in drag. As a result, the lift-to-drag ratio has greatly increased.

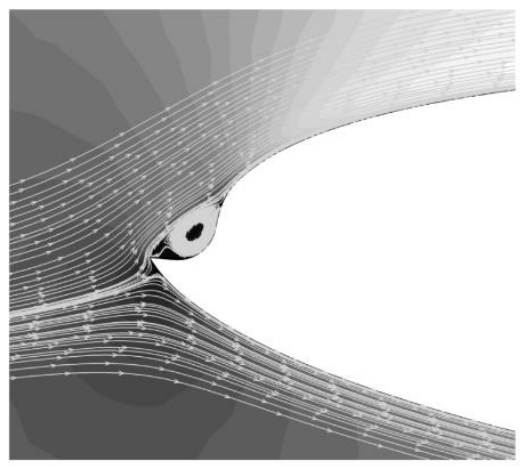

Figure 4.12 The $\mathrm{G}^{4} \mathrm{LTV} 1$ airfoil leading edge vortex, $M=0.7$ and $\operatorname{Re}=9 \times 10^{6}, A o A=5^{\circ}$.

Another remarkable effect of the leading-edge modification is the minor increase in the strength of the shockwave for the full range of angles of attack and the ability to move it forward downstream. Furthermore, $\mathrm{G}^{4} \mathrm{LTV} 1$ modification was able to control the flow separation by reattaching the separated flow from the airfoil upper surface at higher angles of attack even with such a small vortex at the leading edge (see Figs. 4.14 and 4.15).

In other words, the higher the angle of attack, the further the shock moves downstream. keeping the flow attached longer (Fig. 4.16), which is likely the reason behind the lift increase and the notable drag reduction which leads to higher lift-to-drag ratio compared to the baseline airfoil. 

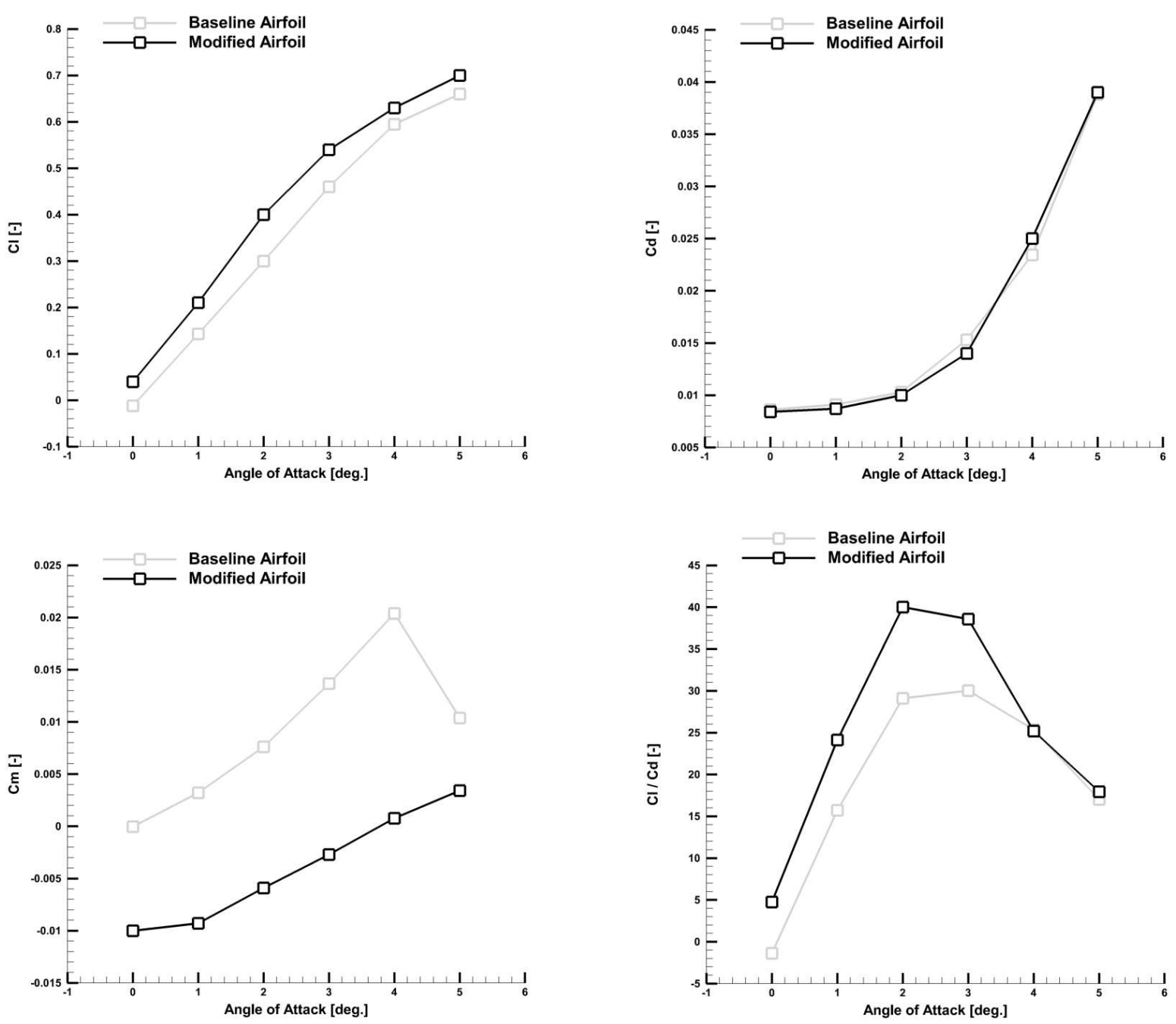

Figure 4.13 Aerodynamic loads of $\mathrm{G}^{4} \mathrm{LTV} 1$ airfoil, $M=0.7$ and $R e=9 \times 10^{6}$.

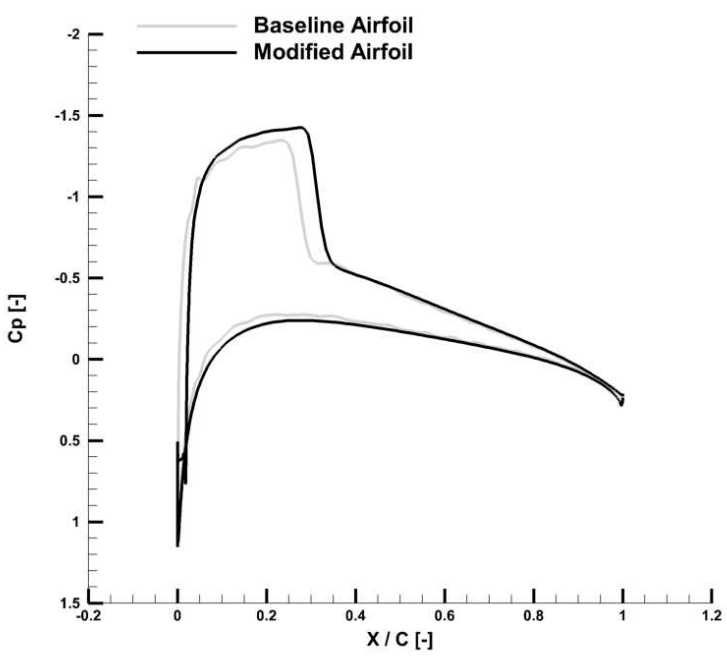

Figure 4.14 Pressure coefficients of $\mathrm{G}^{4} \mathrm{LTV} 1$ airfoil, $M=0.7$ and $R e=9 \times 10^{6}, A o A=2.5^{\circ}$. 

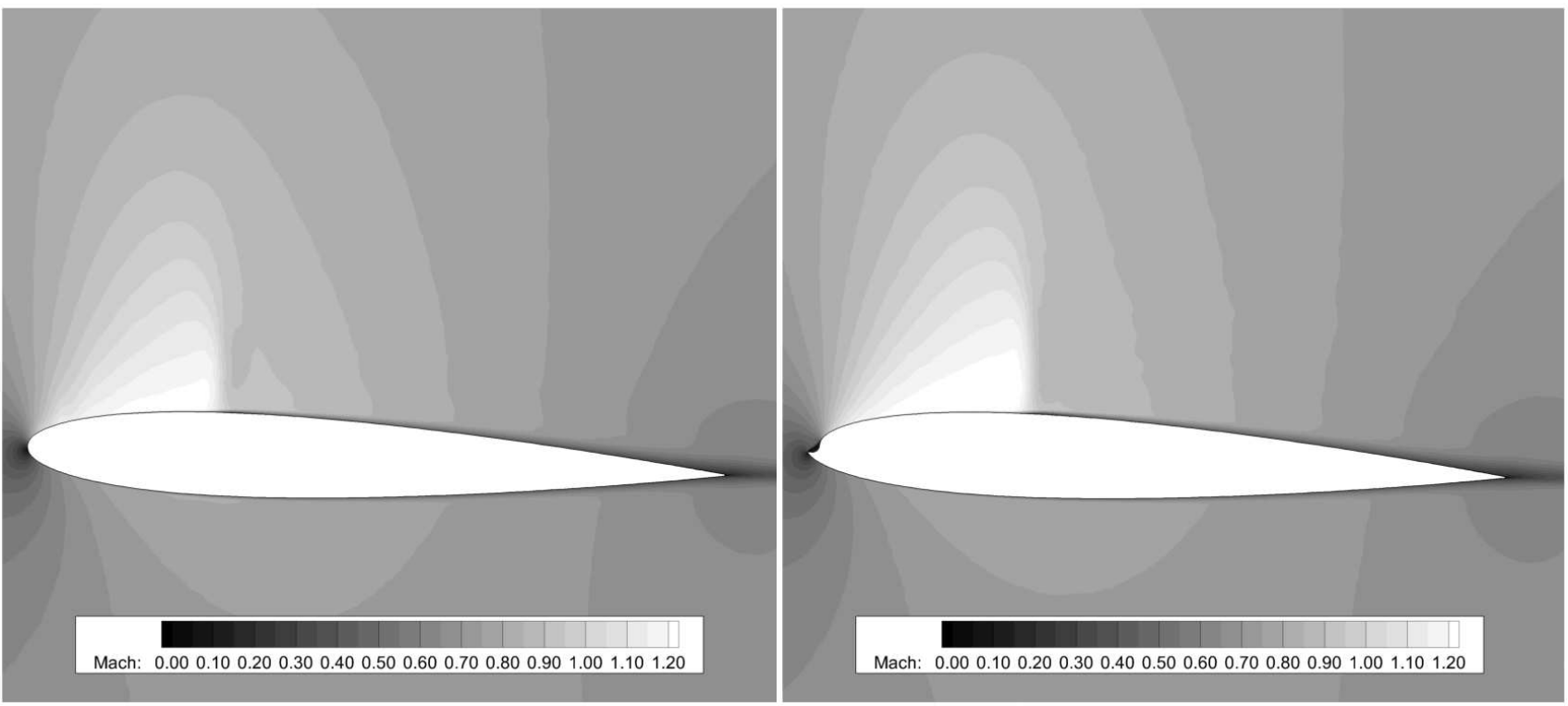

Figure 4.15 Mach number contours, baseline airfoil (left), $\mathrm{G}^{4} \mathrm{LTV} 1$ airfoil (right), $M=0.7$ and $R e=9 \times 10^{6}, A o A=2.5^{\circ}$.

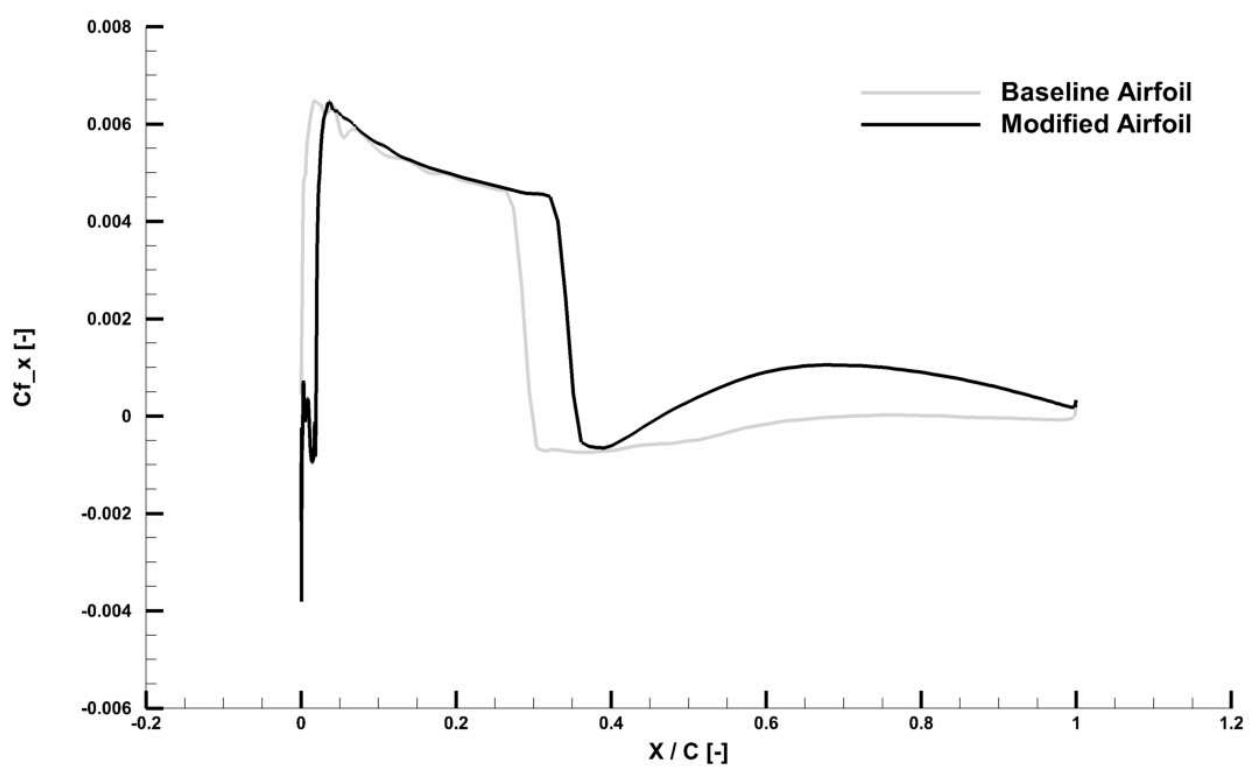

Figure 4.16 Skin friction coefficient (x-component) for the baseline airfoil and the airfoil with $\mathrm{G}^{4} \mathrm{LTV} 1$ modification, upper surface, at $M=0.7$ and $R e=9 \times 10^{6}, C_{l}=0.66$. 


\subsubsection{NASA SC(3)-0712(B) Supercritical Airfoil (Test Case 2)}

In addition to the discussions presented earlier, it was important to test the validity of the conclusions on a realistic supercritical airfoil. Thus, a NASA SC(3)-0712(B) supercritical airfoil was modified with the $\mathrm{G}^{2} \mathrm{U} 3$ and $\mathrm{G}^{4} \mathrm{LTV} 1 \mathrm{i}$ type modifications. According to [89], the test conducted on the baseline NASA SC(3)-0712(B) supercritical airfoil was part of the Advanced Technology Airfoil Tests (ATAT) program. A significant portion of this test was carried out in cooperation with three manufacturers of large commercial transport aircraft (Boeing, Douglas, and Lockheed). One of the main objectives of the ATAT program was to provide the industry with the ability to study their airfoils at flight Reynolds number. Therefore, the NASA SC(3)-0712(B) airfoil was selected to validate the concepts proposed above for their potential use on a conventional transport jet.

\subsubsection{NASA SC(3)-0712(B) with $G^{2} U 3$ Modification}

This is a $2^{\text {nd }}$ generation modification applied to the NASA SC(3)-0712(B) airfoil (Fig. 4.3). It features an upper surface cavity close to the trailing edge, at $0.82 c$ from the leading edge with a depth of $0.4526 t$ and extended to $0.1054 c$.

The aerodynamic results of the modified airfoil are shown in Fig. 4.17 for angles of attack ranged from $0^{\circ}$ to $5^{\circ}$. The modified airfoil increased the lift-to-drag ratio for the full range of the investigated angles of attack as well as reduced the drag and the strength of shockwave when compared to the baseline airfoil. As expected, the airfoil produces a smaller negative pitching moment than its unmodified counterpart, which was the main characteristic of the upper surface modifications in general, as shown previously in Table 
4.2. It is also worth to mention that these results come at virtually no lift penalty (penalty was in the vicinity of $2 \%$ ).

The pressure distribution at an angle of attack $2.5^{\circ}$ (corresponding to cruise) is given in Fig. 4.18. The figure shows that with only a simple modification of the upper surface, the strength of the shockwave can be reduced. The pitching moment also reduced because the lower negative pressure on the upper surface near the trailing edge. This creates a countering moment, opposing the negative nose-up pitching moment.

Fig. 4.19 provides maximum Mach number contours for the investigated flow regimes at angle of attack $2.5^{\circ}$. A careful examination of these figures supports the discussion above and further explain the physics behind the results from the proposed modification. 

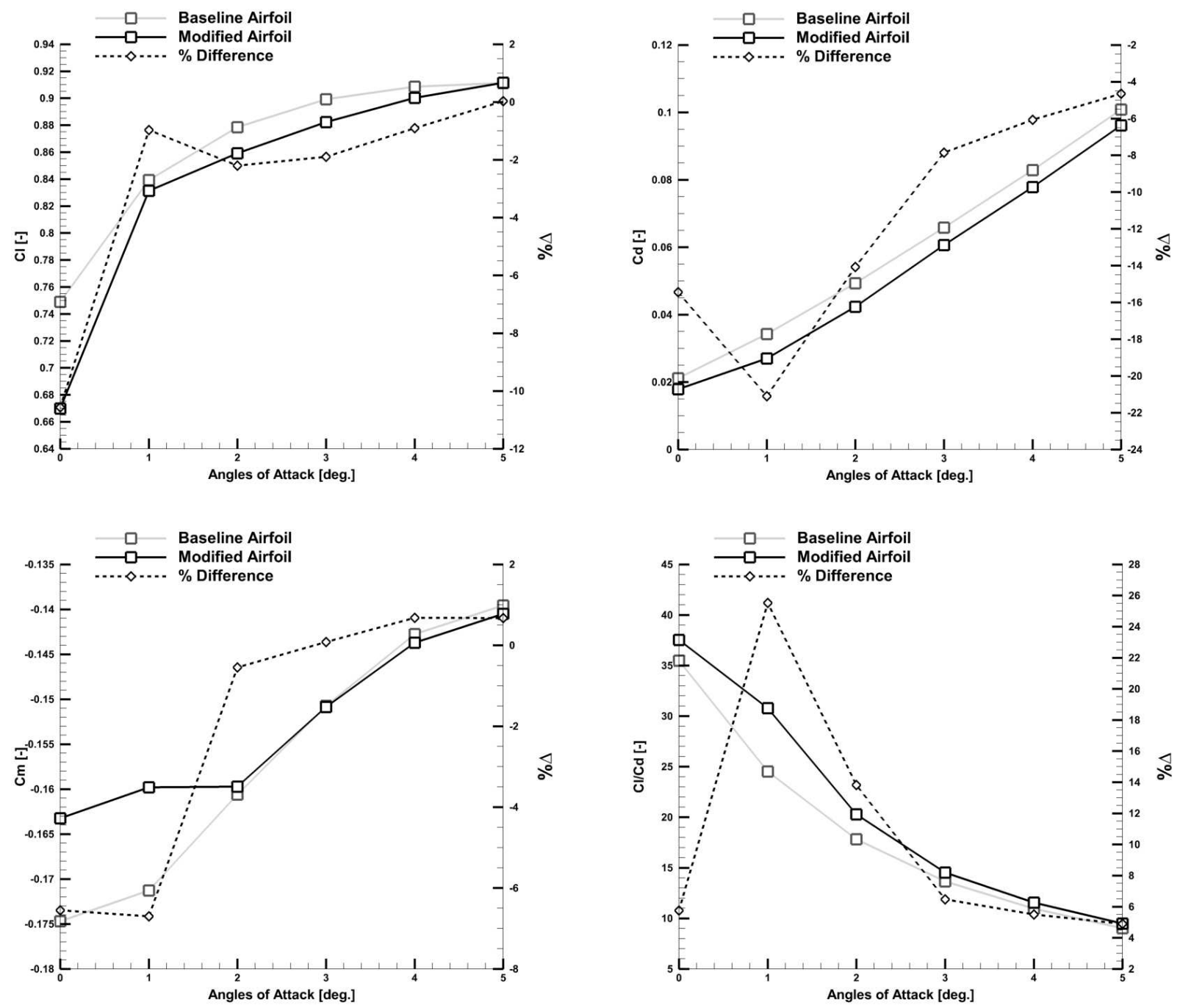

Figure 4.17 Aerodynamic loads for the NASA SC(3)-0712(B) airfoil with a $\mathrm{G}^{2} \mathrm{U} 3$ modification, $M=0.78$ and $R e=30 \times 10^{6}$. 


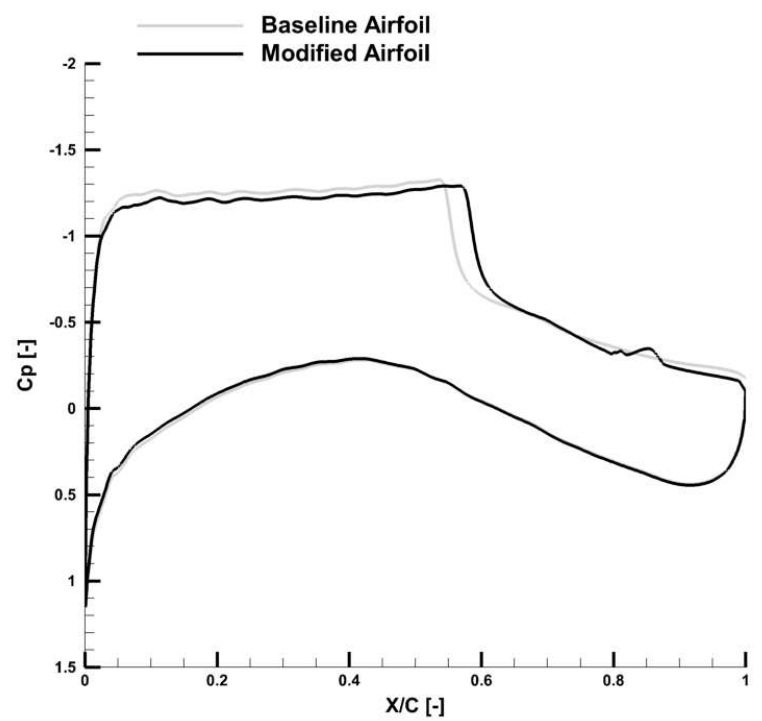

Figure 4.18 Pressure coefficients for the NASA SC(3)-0712(B) airfoil with a $\mathrm{G}^{2} \mathrm{U} 3$ modification, $M=0.78$ and $R e=30 \times 10^{6}, A o A=2.5^{\circ}$.
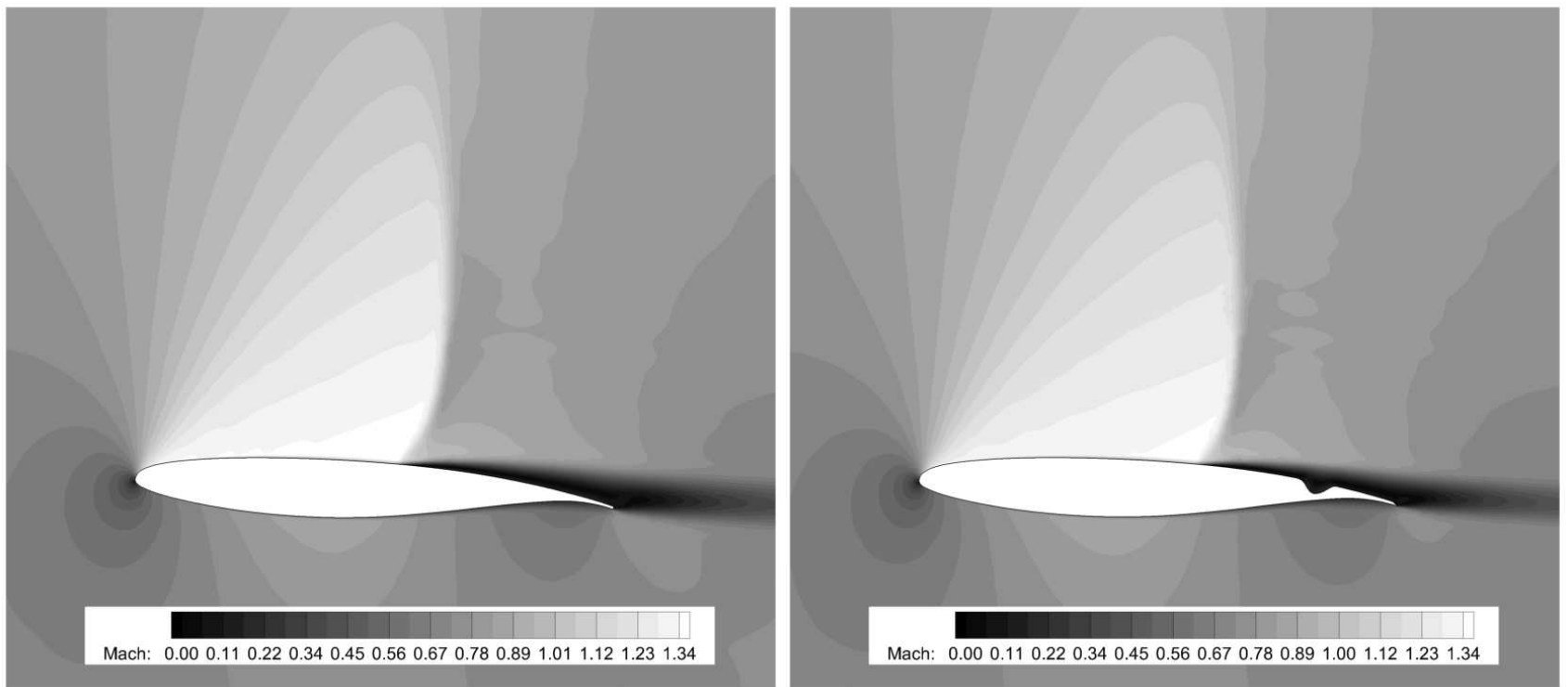

Figure 4.19 Mach number contours, NASA SC(3)-0712(B) baseline airfoil (left), with a $\mathrm{G}^{2} \mathrm{U} 3$ modification (right), $M=0.78$ and $R e=30 \times 10^{6}, A o A=2.5^{\circ}$. 


\subsubsection{NASA SC(3)-0712(B) with $G^{4} L T V 1 i$ Modification}

The geometry of the modified NASA SC(3)-0712(B) airfoil with the $\mathrm{G}^{4} \mathrm{LTV} 1 \mathrm{i}$ modification is given in Fig. 4.3. It is an inverted version of the $\mathrm{G}^{4} \mathrm{LTV} 1$ modification of the previously discussed modification used on the NACA 0012 airfoil (for Test Case 1). Unlike the NACA 0012 airfoil with $\mathrm{G}^{4} \mathrm{LTV} 1$ modification, the vortex this time is in contact with the lower surface of the airfoil.

Fig. 4.20 illustrates the aerodynamic results of the modified NASA SC(3)0712(B) airfoil compared to the baseline airfoil. As can be seen, the results prove that the proposed modifications in this thesis not only work on symmetrical airfoils, such as the NACA 0012 with a specific flow regime (see Table 4.2) but bring similar advantages on one of the most modern airfoils that used in the industry for large transport aircraft.

The pressure distribution of the modified NASA SC(3)-0712(B) airfoil at angle of attack $2.5^{\circ}$ is also compared to the baseline airfoil in Fig. 4.21.

From Figs. 4.21 and 4.22, one can clearly observe that the effect of the modification is that it reduces the strength of the shockwave on the upper surface of the airfoil. Hence, a noteworthy drag reduction occurs, with only a small penalty in lift (mostly below 10\%). However, the lift-to-drag ratio significantly increases for the full range of angles of attack, along with a remarkable deduction in pitching moment. The low pitching moment leads for lower trim drag from the horizontal tail. Combining all the improvements above, the proposed modification has the potential to yield better flight performance and significant reduction in the fuel consumption during cruise flight phase. 

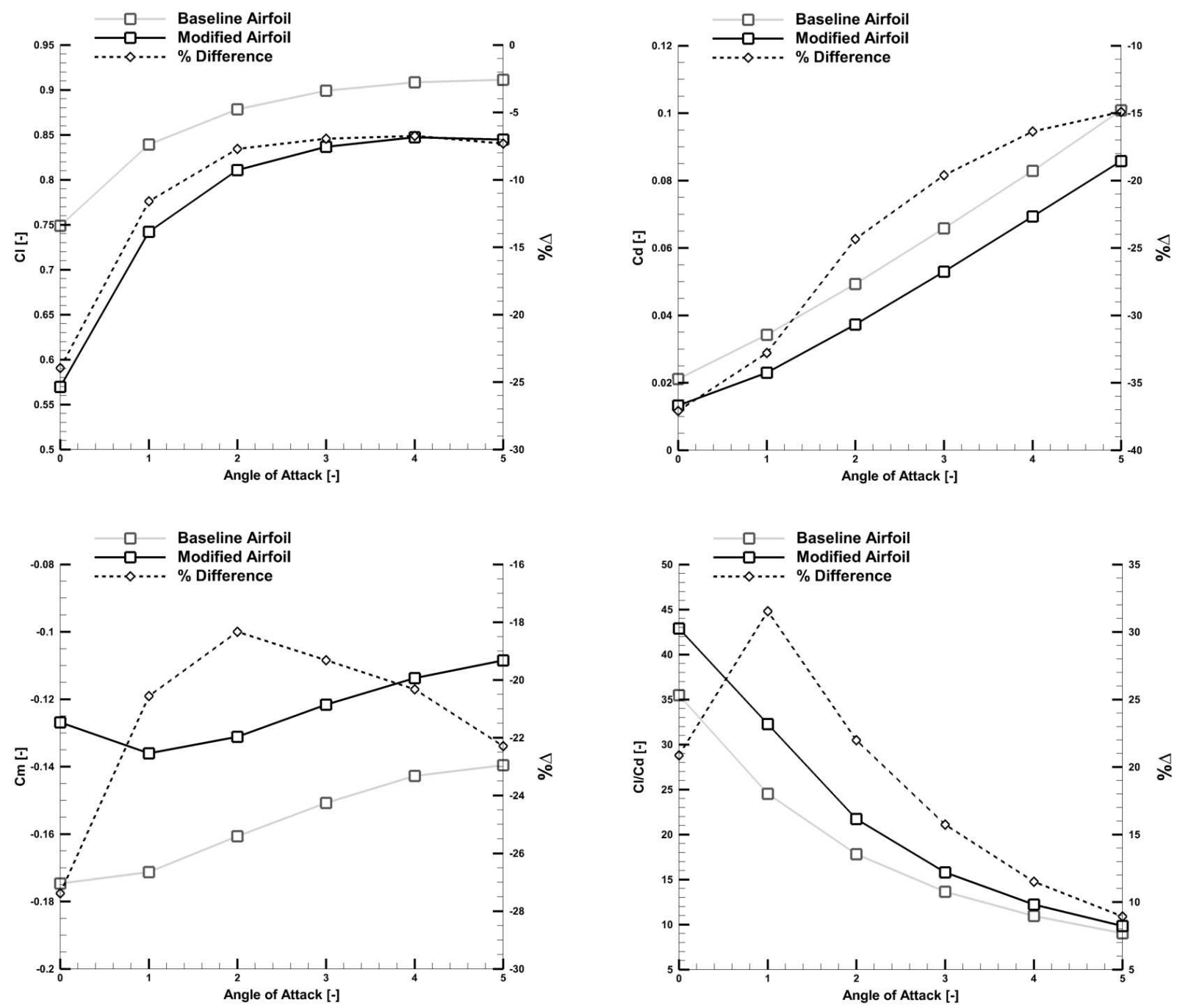

Figure 4.20 Aerodynamic loads for the NASA SC(3)-0712(B) airfoil with a $\mathrm{G}^{4} \mathrm{LTV} 1 \mathrm{i}$ modification, $M=0.78$ and $R e=30 \times 10^{6}$. 


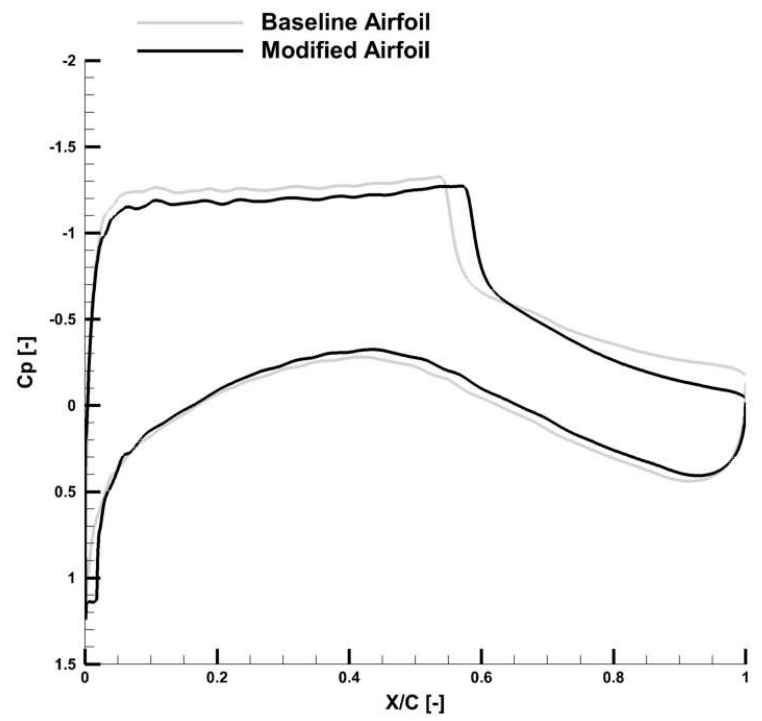

Figure 4.21 Pressure coefficients for the NASA SC(3)-0712(B) airfoil with a $\mathrm{G}^{4} \mathrm{LTV} 1 \mathrm{i}$ modification, $M=0.78$ and $R e=30 \times 10^{6}, A o A=2.5^{\circ}$.
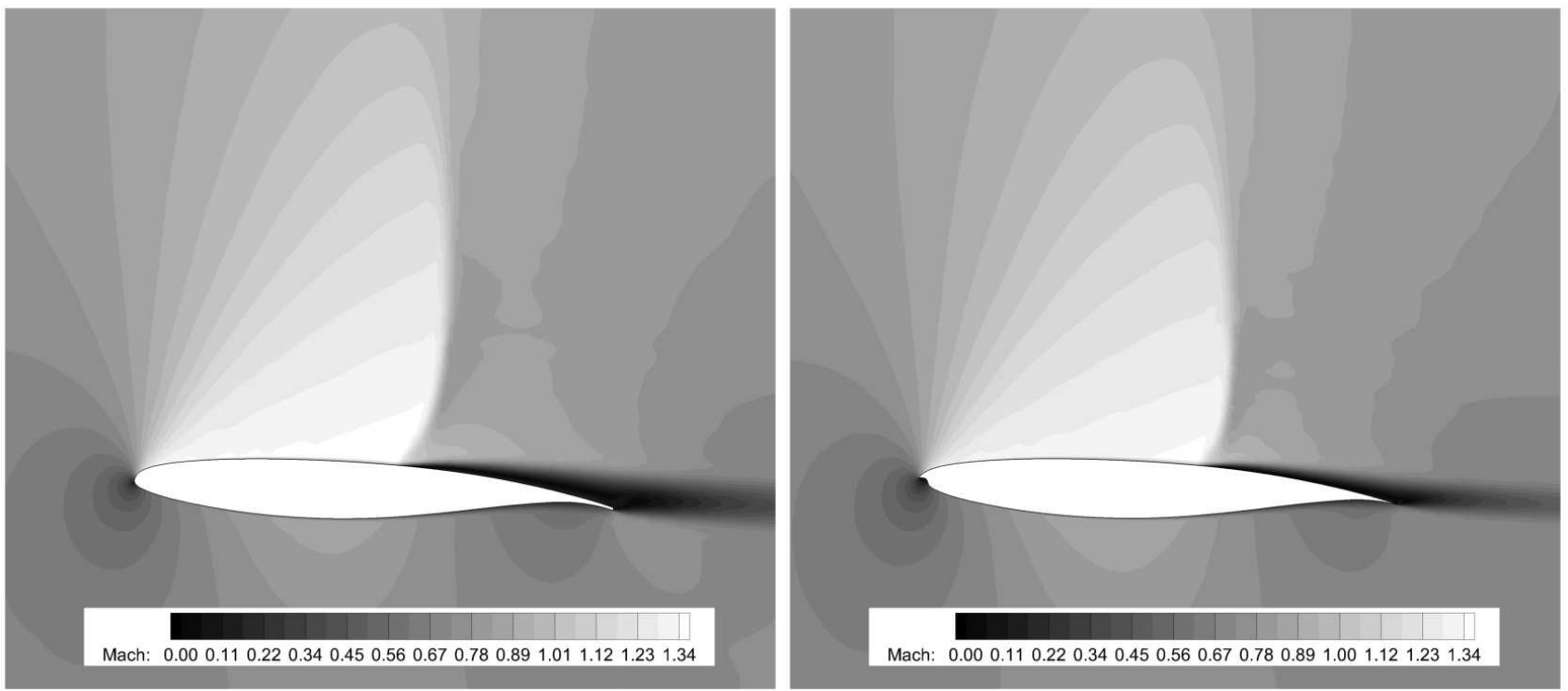

Figure 4.22 Mach number contours, NASA SC(3)-0712(B) baseline airfoil (left), with a $\mathrm{G}^{4} \mathrm{LTV} 1 \mathrm{i}$ modification (right), $M=0.78$ and $R e=30 \times 10^{6}, A o A=2.5^{\circ}$. 


\subsubsection{Passive Flow Control Robustness Test}

Since the proposed surface modifications could be sensitive to changes in the flight conditions, such as Mach number changes during take-off and landing, the proposed modifications were further investigated for these flight conditions.

The baseline NASA SC(3)-0712(B) airfoil and modified versions with $\mathrm{G}^{2} \mathrm{U} 3$ and $\mathrm{G}^{4} \mathrm{LTV} 1 \mathrm{i}$ modifications were analyzed in this section for Mach numbers 0.3 to 0.86 at angle of attack $2.5^{\circ}$. Fig. 4.23 shows the drag as well as the percentage reduction of drag against Mach number at fixed angle of attack using the proposed modification. The selected Mach numbers' range is ideal for take-off, cruise and landing phases of a typical commercial jet [108].

As can be seen from Fig. 4.23, the deduction in the drag increase with the increase in Mach number regardless the type of the modification used, which indicates a possible better flight performance and a promising reduction in the fuel consumption, especially during the cruise phase, hence, the proposed modification could significantly enhance the aerodynamic characteristics of fixed-wing aircraft. 

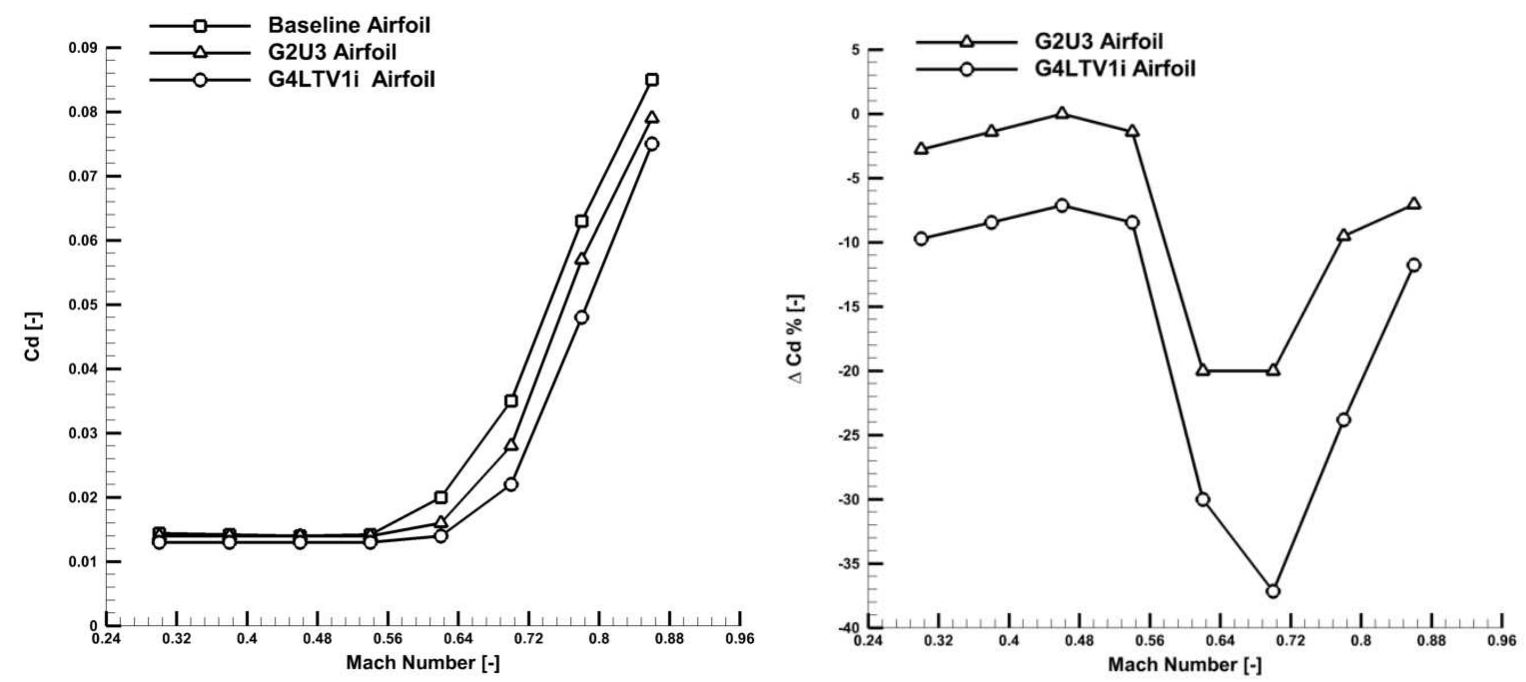

Figure 4.23 Drag-Mach number graphs for the NASA SC(3)-0712(B) supercritical airfoil compared to $\mathrm{G}^{2} \mathrm{U} 3$ and $\mathrm{G}^{4} \mathrm{LTV} 1 \mathrm{i}$ modification at $A o A=2.5^{\circ}$.

\subsection{Passive Flow Control for Mitigation of 2D Dynamic Stall}

A NACA 0012 airfoil was used as the "baseline" airfoil in this section, because a wide range of dynamic stall experimental data is available for it. As before, surface-based passive flow control was created by modifying one of the airfoil surfaces by implementing a trapped vortex generator in it.

\subsubsection{Constant Freestream Dynamic Stall}

CFD simulations of 2D dynamic stall under a constant freestream of 0.3 Mach number (subsonic) were completed with the numerical parameters described earlier in Chapter 3. Only 26 airfoils form those shown in Fig. 4.2 were simulated, because the $4^{\text {th }}$ Generation modifications were solely designed for $0.7 \leq \mathrm{M} \leq 0.9$. Hence, these were excluded from this section. The summary of the simulation results is shown in Table 4.3. 
The parameters, on which basis the performance of an airfoil was determined were: a) the peak lift coefficient $\left.\left(C_{l, \max }\right), \mathrm{b}\right)$ the peak drag coefficient $\left(C_{d, \max }\right)$, and c) the peak negative pitching moment $\left(C_{m, \max }\right)$.

Recall, the goal was to design such a passive flow control concept, which does not deteriorate the lift characteristics of the original airfoil significantly, while offering significant improvements in drag and pitching moment, i.e. which decreases peak drag and peak negative pitching moments, especially around the maximum angle of attack region, where dynamic stall occurs. Reduction of the pitching moment is desired, since this translates to vibratory loads in the pitch links during forward flight. As can be seen from Table 4.3, some of the airfoils perform remarkably well. For example, nearly all upper surface modifications reduce the peak negative pitching moment and peak drag by as much as $60 \%$ and $30 \%$, respectively, while sacrificing only about $5 \sim 10 \%$ of the peak lift. On the other hand, the lower surface modifications can increase the peak lift by about $10-16 \%$, but at the expense of a minor increase in peak drag and peak negative pitching moment as well.

To help increase the understanding of the potentials of the new designs, the aerodynamic loads will be presented for both static and unsteady test cases. Static means slow change in the angle of attack, which means that flow phenomenon behaves in a quasi-steady manner [1]. Whereas, unsteady means a fast change in the angle of attack, which causes the unsteady nature of the flow phenomenon to become important [1], this case also known as dynamic stall case.

The search for the reasons behind the presented results from the novel designs was conducted through detailed analysis of the flow via flow visualizations for all 
airfoils. However, due to space constraints, only a selection of such detailed results is shown in this section for 3 airfoils (indicated by gray arrows in Table 4.3).

Table 4.3 Results summary of the modified NACA 0012 airfoil.

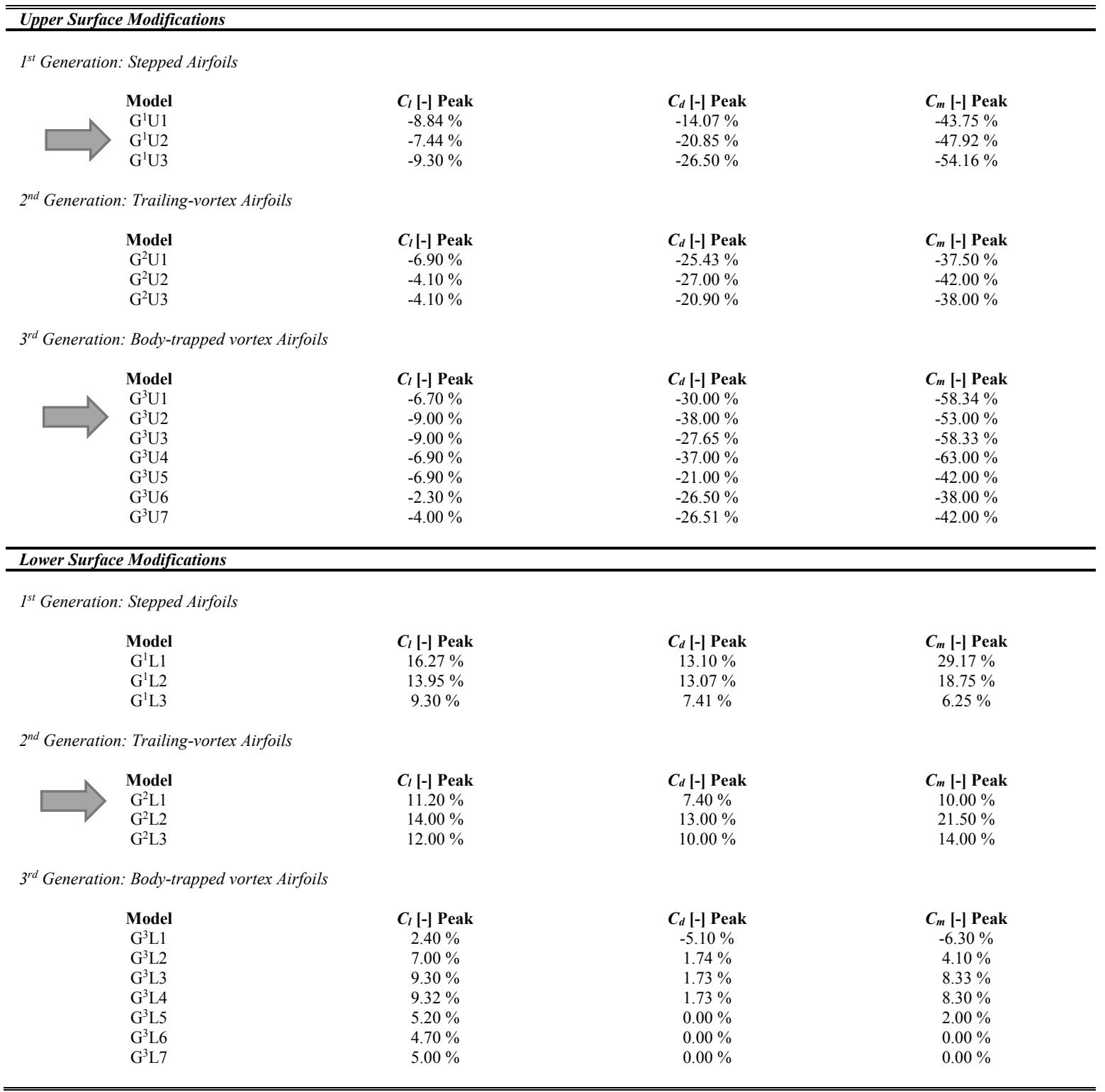

Fig. 4.24 illustrates a NACA 0012 airfoil with the selected modifications from the table above. 
Figure 4.24 NACA 0012 airfoil with the selected modifications applied.

\subsubsection{NACA 0012 with $G^{1} U_{2}^{2}$ Modification}

The $\mathrm{G}^{1} \mathrm{U} 2$ airfoil has a BFS on the upper surface, starting at $0.5 c$ and extending to the trailing edge. The depth of the step is $0.19 t$. Fig. 4.24 above illustrates the shape of the airfoil. From Table 4.3, one can see that this airfoil exhibits outstanding ability to mitigate the negative effects of dynamic stall by significantly decreasing drag and pitching moment (by about $21 \%$ and $48 \%$, respectively) with only a moderate loss in the peak lift (-7\%). Fig. 4.25 presents the results of the aerodynamic loads compared to the baseline airfoil for both static stall and dynamic stall conditions. It is worth to note that in terms of the static aerodynamic results, the $\mathrm{G}^{1} \mathrm{U} 2$ airfoil demonstrates better aerodynamic behavior than the baseline airfoil in terms of better stall resistance, the increase in the range of the operational angles of attack as well as lower drag at higher angles of attack.

For the dynamic stall case, the modified airfoil shows substantial increase in lift during the downstroke phase of the oscillation, between angles of attack of $24^{\circ}$ to $15^{\circ}$.

The pressure coefficients, instantaneous streamlines and velocity of the baseline and modified airfoils are compared in Fig. 4.26. A careful examination of these figures 
allows to conclude that the presence of the vortex inside the step has almost no effect on the flow at lower angles of attack. However, its effect becomes significant at higher angles of attack.

The driving mechanism of the pitching moment reduction can be explained via examining the flow visualization and pressure distribution plots in Fig. 4.26. Note that the flow is virtually unchanged at low angles of attacks (Frames A-C). The $C_{p}$ plots, however, start to significantly deviate from Frame D. A dynamic stall vortex is created on both airfoils; however, its extent is confined to the region before the step on the modified airfoil. This is likely due to the step geometry preventing some of the fluid to recirculate back to the vortex. This leads to a smaller and likely weaker dynamic stall vortex for the modified airfoil, which - when convected downstream (Frames E, F) - will generate less suction on the upper surface towards the trailing edge. This, in turn, reduces the negative pitching moment significantly (Frames D-G). The reduced size and strength of the dynamic stall vortex will lead to less drag too, although it is speculated that perhaps the fluid in the dynamic stall vortex inside the step might impact the flow in a way so that it creates a forward pointing force. This might also contribute to the drag reduction.

The velocity reduction on the upper surface is likely due to the interaction between the vortices shed from the airfoil upper surface as well as the vortex expansion inside the step at higher angles of attack. This latter effect is in line with the conclusions made by Al-Jaburi in his experiments on several stepped airfoils at low freestream Mach number $[22]$.

The opposite behavior can be observed during the downstroke phase. The surface velocity behind the step is larger than that for the baseline airfoil, which leads to lower 
pressure and increased lift during the downstroke phase, as shown in Frame H on Figs.

\subsection{5 and 4.26 .}
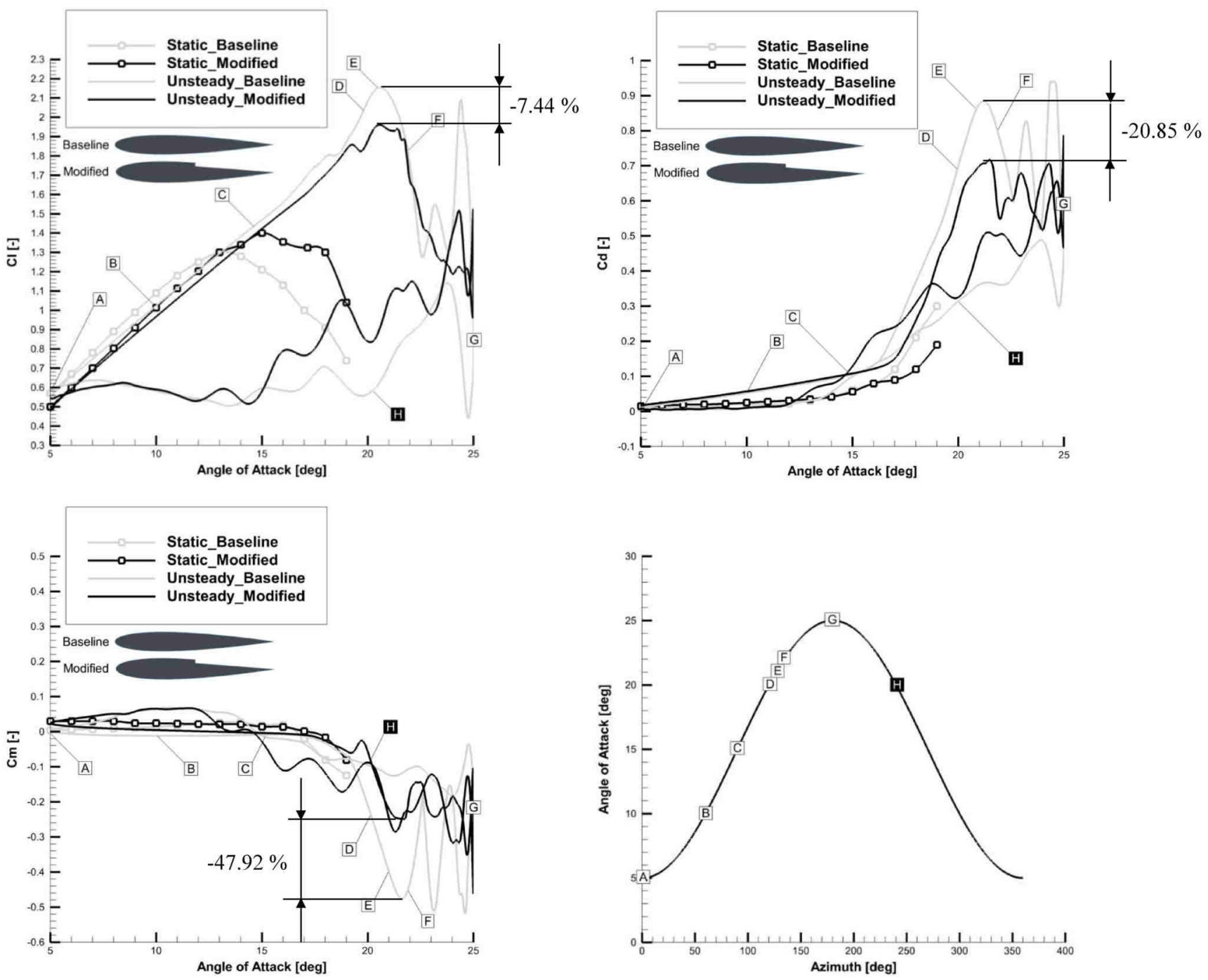

Fig. 4.25 Aerodynamic loads for the $\mathrm{G}^{1} \mathrm{U} 2$ airfoil with the selected azimuthal positions.

$$
M=0.3, \operatorname{Re}=3.76 \times 10^{6} \text { and } \alpha=15^{\circ} \pm 10^{\circ} .
$$



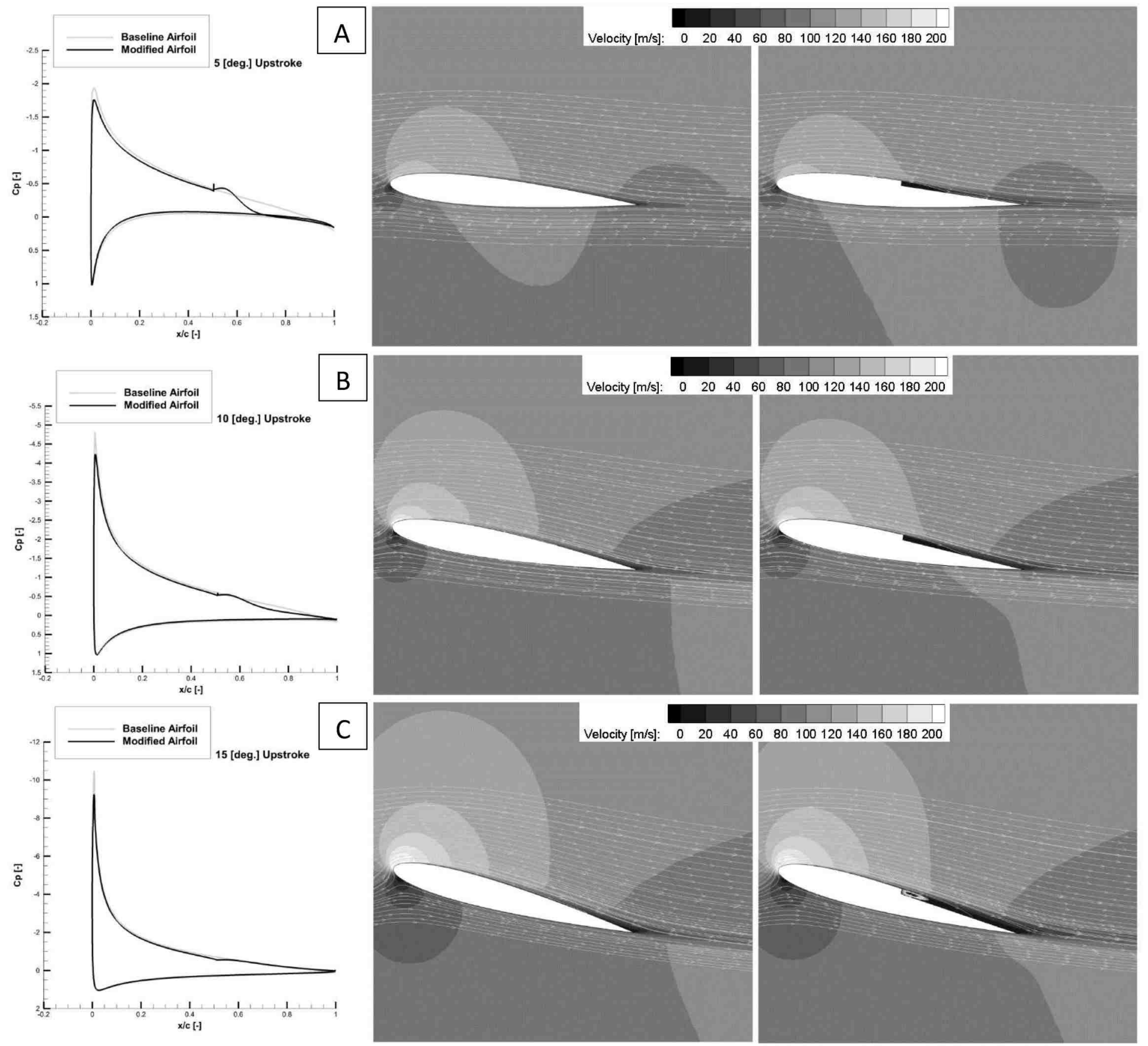

Fig. 4.26 Pressure coefficient, instantaneous streamlines and velocity contours for the

NACA 0012 airfoil and the $\mathrm{G}^{1} \mathrm{U} 2$ airfoil. $M=0.3, R e=3.76 \times 10^{6}$ and $\alpha=15^{\circ} \pm 10^{\circ}$. 

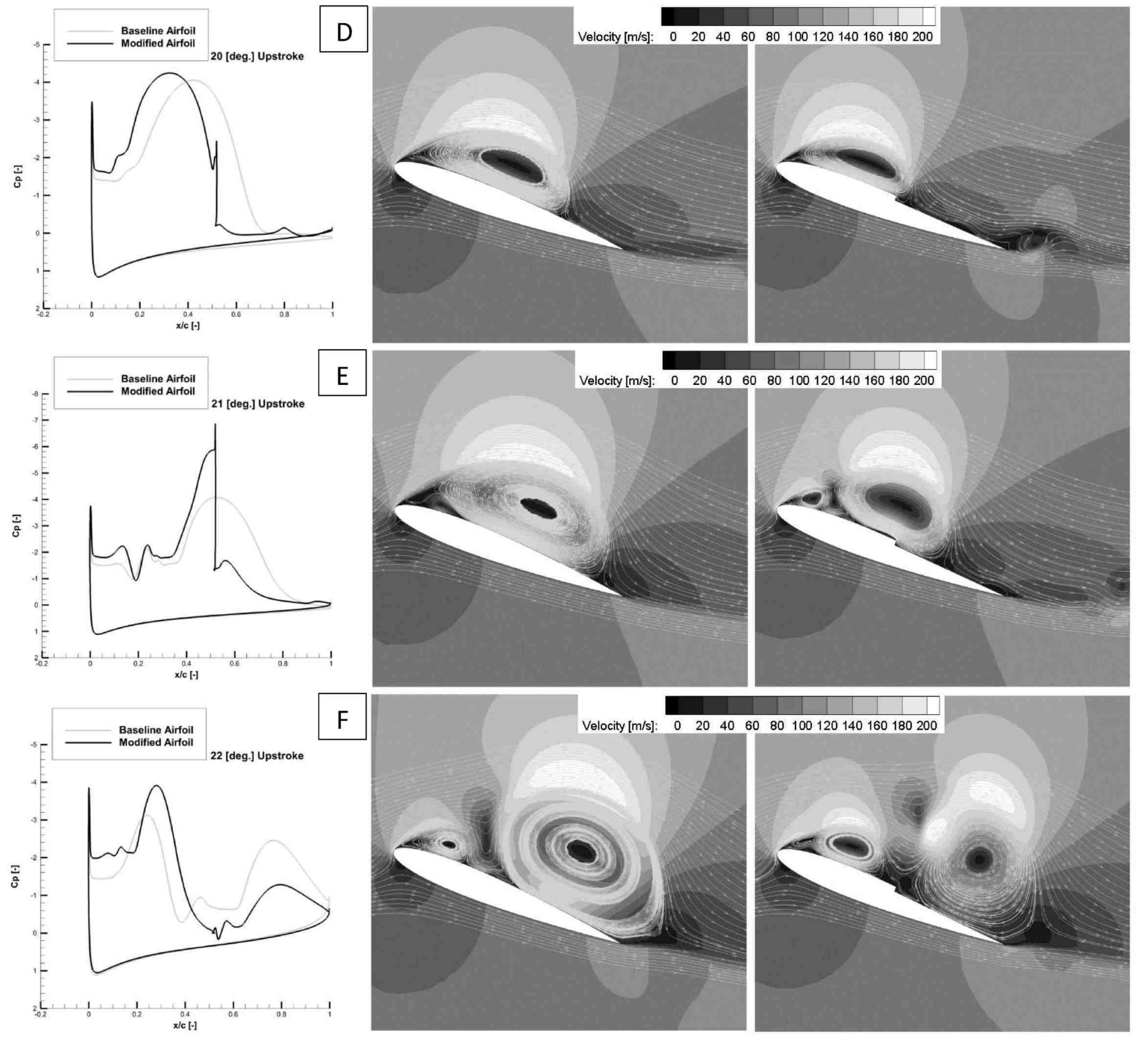

Figure 4.26 (Continued) Pressure coefficient, instantaneous streamlines and velocity contours for the NACA 0012 airfoil and the $\mathrm{G}^{1} \mathrm{U} 2$ airfoil. $M=0.3, R e=3.76 \times 10^{6}$ and $\alpha=$ $15^{\circ} \pm 10^{\circ}$ 

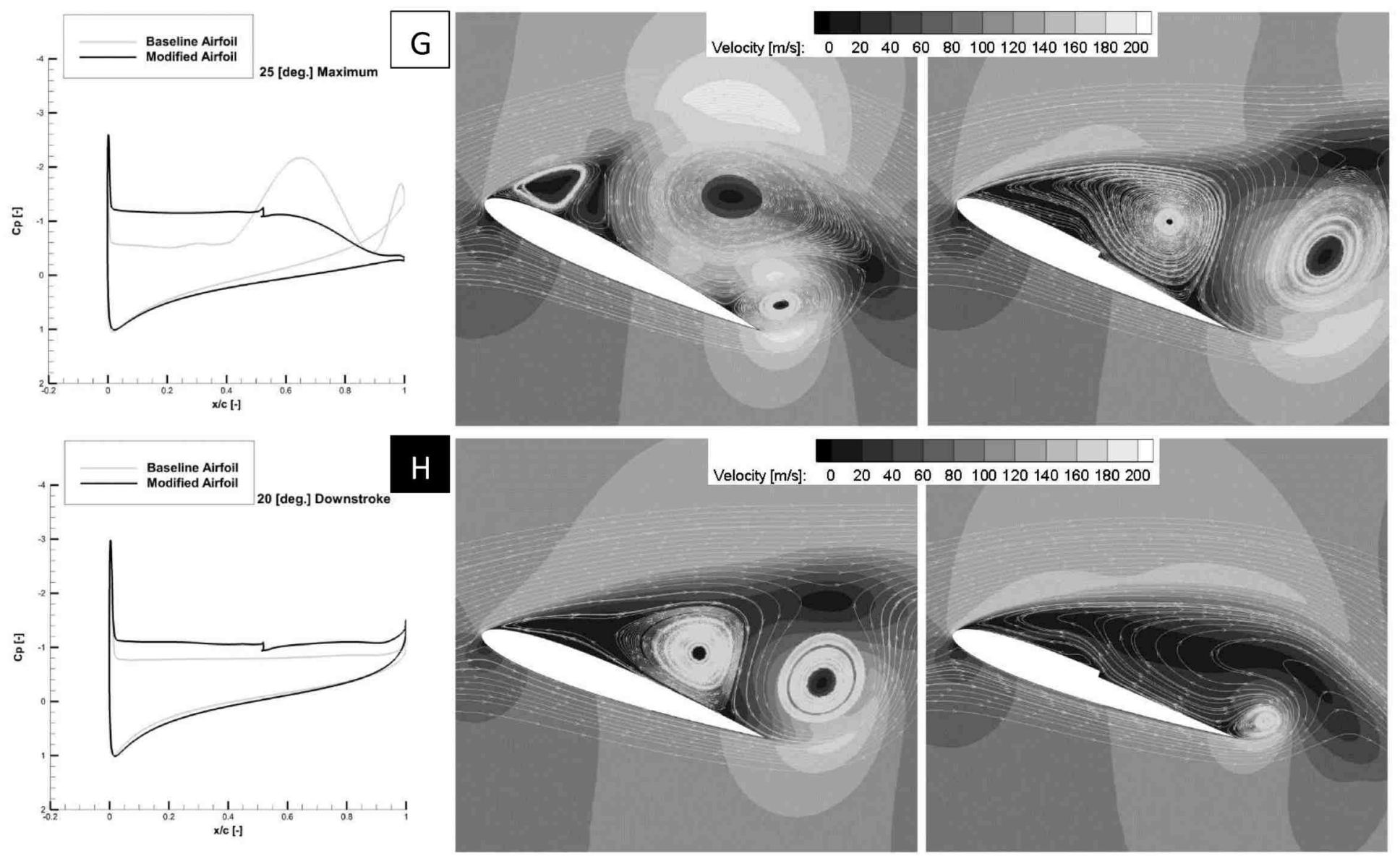

Figure 4.26 (Continued) Pressure coefficient, instantaneous streamlines and velocity contours for the NACA 0012 airfoil and the $\mathrm{G}^{1} \mathrm{U} 2$ airfoil. $M=0.3, \operatorname{Re}=3.76 \times 10^{6}$ and $\alpha=$

$$
15^{\circ} \pm 10^{\circ}
$$

\subsubsection{NACA 0012 with $G^{3} U 2$ Modification}

This modification geometry is able to control the shedding of the dynamic stall vortex over the upper surface via trapping some of this vortex in the cavity. The airfoil with this modification has the cavity at $0.5 c$ with a depth of $0.18 t$ and extended only $0.164 c$ towards the trailing edge. Fig. 4.24 illustrates the shape of $\mathrm{G}^{3} \mathrm{U} 2$ airfoil, while Figs. 4.27 and 4.28 show the aerodynamic loads and the pressure coefficient, 
instantaneous streamlines and velocity contours, respectively, compared to the baseline NACA 0012 airfoil.

Although the geometry of the vortex generator is very different from the previously analyzed $\mathrm{G}^{1} \mathrm{U} 2$ airfoil, it has preserved that desired characteristic of the airfoil, that the flow is not affected at low angles of attack. Thus, from the aerodynamic loads shown on Fig. 4.27, it is clear that in the static test case, this new design outperforms the baseline airfoil again, since the $\mathrm{G}^{3} \mathrm{U} 2$ airfoil has a higher stall angle as well as at higher angles of attack, this new airfoil has higher lift and pitching moment with lower drag compared to the baseline airfoil. Moreover, one can observe that up to an angle of attack $20^{\circ}$ the two airfoils behave practically identically, after which the drag and pitching moment become significantly reduced (by $38 \%$ and $53 \%$, respectively), while sacrificing only $9 \%$ of peak lift. The reasons for the reduction in pitching moment become clear after examining the pressure coefficients in frames D to F of Fig. 4.28. In these frames, one can observe that the vortex generator modifies the pressure distribution in a way so that most of the lift remains very close to the pitching axis. As a result, it will prevent the occurrence of large nose down pitching moment. The velocity contours and streamlines of Fig. 4.28 allow to understand the reason for this effect: it is the cavity trapping some of the fluid entrained in the dynamic stall vortex, as well as keeping it confined to the front half of the airfoil upper surface.

At the same time, because the cavity has the ability to trap part/a portion of the dynamic stall vortex, it will likely decrease its strength, and this will reduce the drag dramatically (see Fig. 4.28). The reason behind the reduction the drag is similar to the analysis given for the $G^{1} U 2$ airfoil. Although there is a loss in lift because of this phenomenon ( $9 \%$ ), this appears to be an acceptable penalty for a more than $50 \%$ decrease 
in the pitching moment which may lead to the reduction of pitch link vibratory loads.
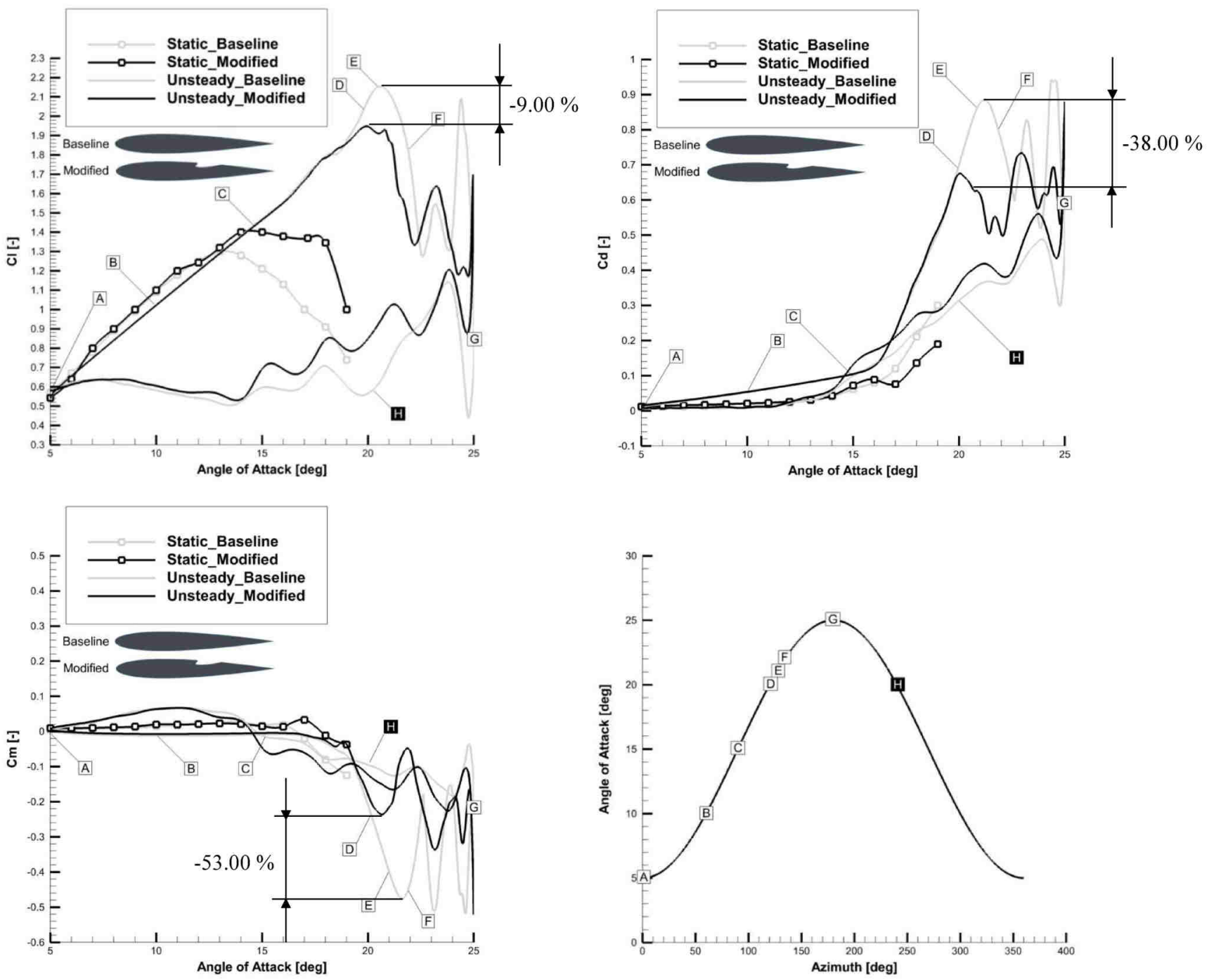

Figure 4.27 Aerodynamic loads for the $\mathrm{G}^{3} \mathrm{U} 2$ airfoil with the selected azimuthal positions. $M=0.3, \operatorname{Re}=3.76 \times 10^{6}$ and $\alpha=15^{\circ} \pm 10^{\circ}$. 

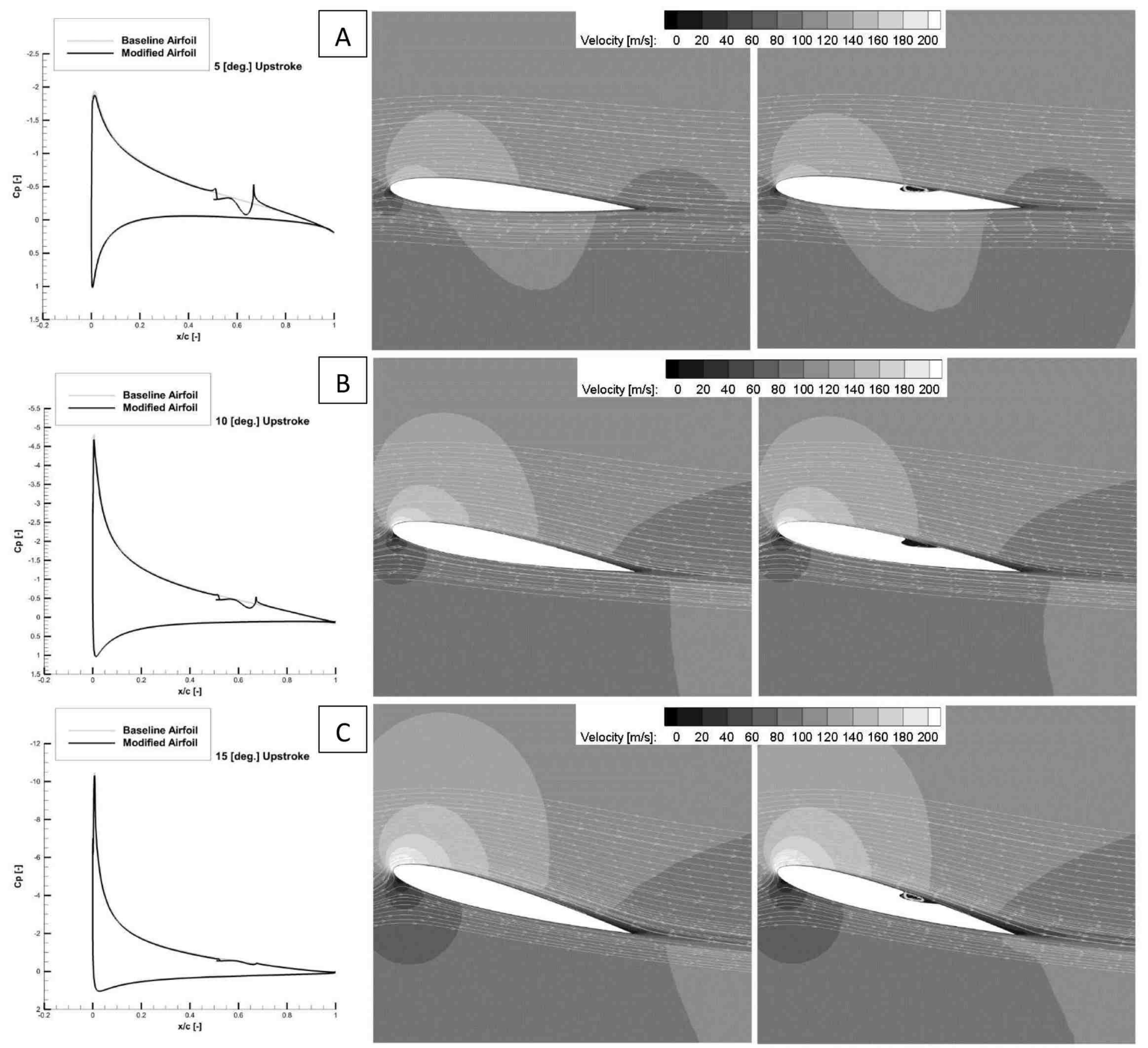

Figure 4.28 Pressure coefficient, instantaneous streamlines and velocity contours for the NACA 0012 airfoil and the $\mathrm{G}^{3} \mathrm{U} 2$ airfoil. $M=0.3, R e=3.76 \times 10^{6}$ and $\alpha=15^{\circ} \pm 10^{\circ}$. 

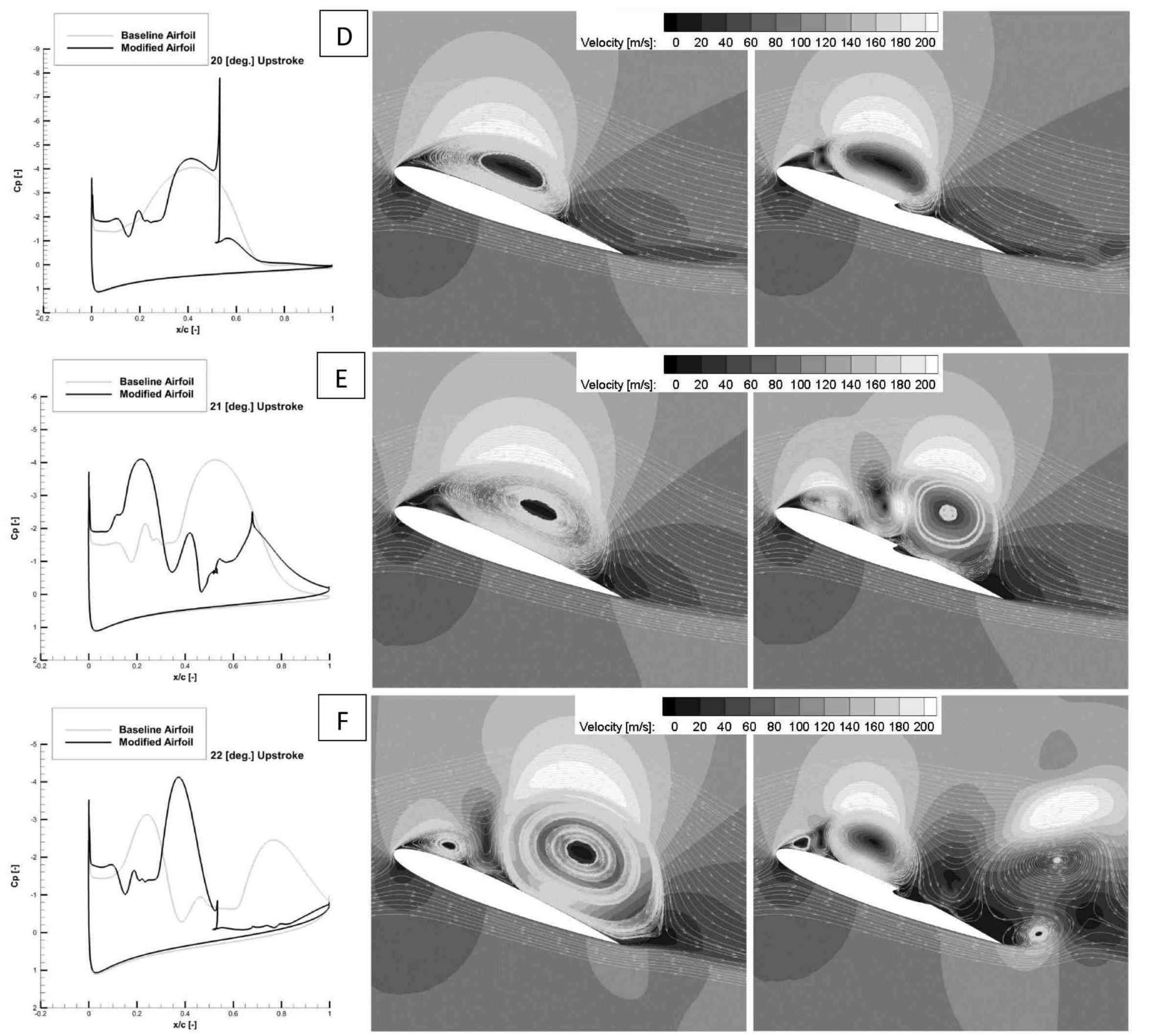

Figure 4.28 (Continued) Pressure coefficient, instantaneous streamlines and velocity contours for the NACA 0012 airfoil and the $\mathrm{G}^{3} \mathrm{U} 2$ airfoil. $M=0.3, R e=3.76 \times 10^{6}$ and $\alpha=$ $15^{\circ} \pm 10^{\circ}$. 

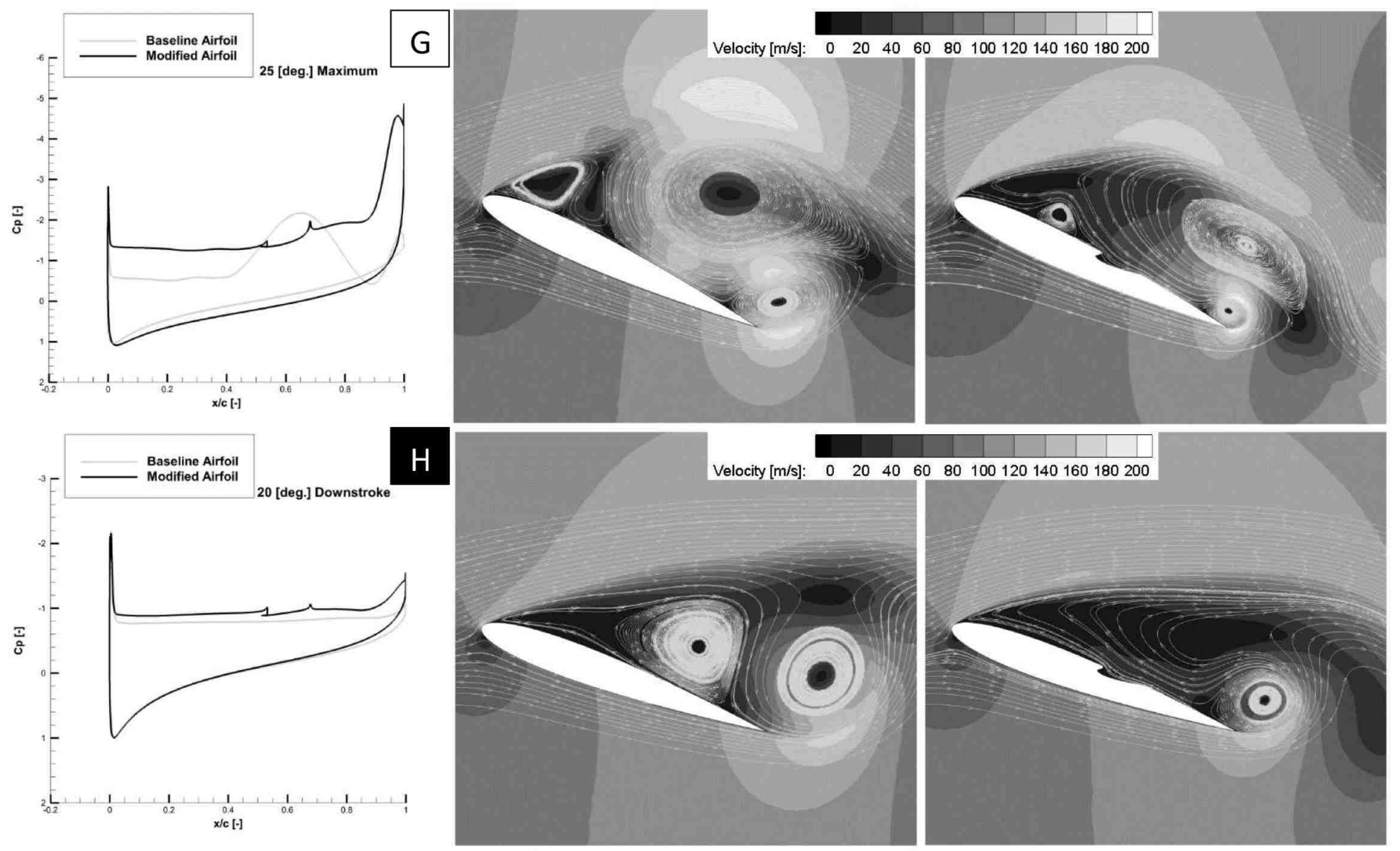

Figure 4.28 (Continued) Pressure coefficient, instantaneous streamlines and velocity contours for the NACA 0012 airfoil and the $\mathrm{G}^{3} \mathrm{U} 2$ airfoil. $M=0.3, \operatorname{Re}=3.76 \times 10^{6}$ and $\alpha=$

$$
15^{\circ} \pm 10^{\circ}
$$

\subsubsection{NACA 0012 with $G^{2} L 1$ Modification}

A lower surface modification, termed $\mathrm{G}^{2} \mathrm{L1}$, will be discussed in detail as well. This has a small step-like, inclined surface near the trailing edge, starting at $0.9 c$. The choice of this geometry was dictated by structural design considerations: the smaller the modification, the better from the structural point of view. The STVG is on the lower surface of the airfoil with a depth of $0.25 t$, (relative to the local thickness, where the 
modification starts). Fig. 4.24 previously illustrated the geometry of NACA 0012 airfoil with the $\mathrm{G}^{2} \mathrm{~L} 1$ modification.

The aerodynamic loads are shown in Fig. 4.29. It is clear from this that the $\mathrm{G}^{2} \mathrm{~L} 1$ airfoil is superior to the baseline airfoil in terms of the lift characteristics at less than $10 \%$ penalty only in drag or pitching moment. For static test, the airfoil generates higher lift for the full range of angles of attack and increases $C_{l, \max }$. Yet, this increased lift comes at lower drag than for the baseline airfoil. For dynamic stall case, the peak lift increased by about $11 \%$ improving considerably the stall characteristics.

This could be explained by examining the pressure distributions in Fig. 4.30. The airfoil has the ability to preserve the pressure distribution of the baseline airfoil on most of its geometry except at the step, at this location, a noteworthy increase in the pressure on the lower surface is observed at practically all angels of attack. Moreover, from angle of attack $21^{\circ}$, the velocity on the upper surface starts to significantly increase, providing more suction, which is the reason behind the lift improvement compared to the baseline airfoil.

These improvements come at nearly no penalty in drag and pitching moment. The increase in the drag and pitching moment (specifically after $20^{\circ}$ ) is likely associated with the minor increase in velocity due to the considerable vortex expansion on the upper surface as a result of the modification of the lower surface of the airfoil [22], see Fig. 4.30. Moreover, better flow reattachment was found starting from $25^{\circ}$ and during the downstroke phase.

The most interesting observation here is that with a truly minor modification of the lower surface, one can affect the flow significantly on the upper surface. 

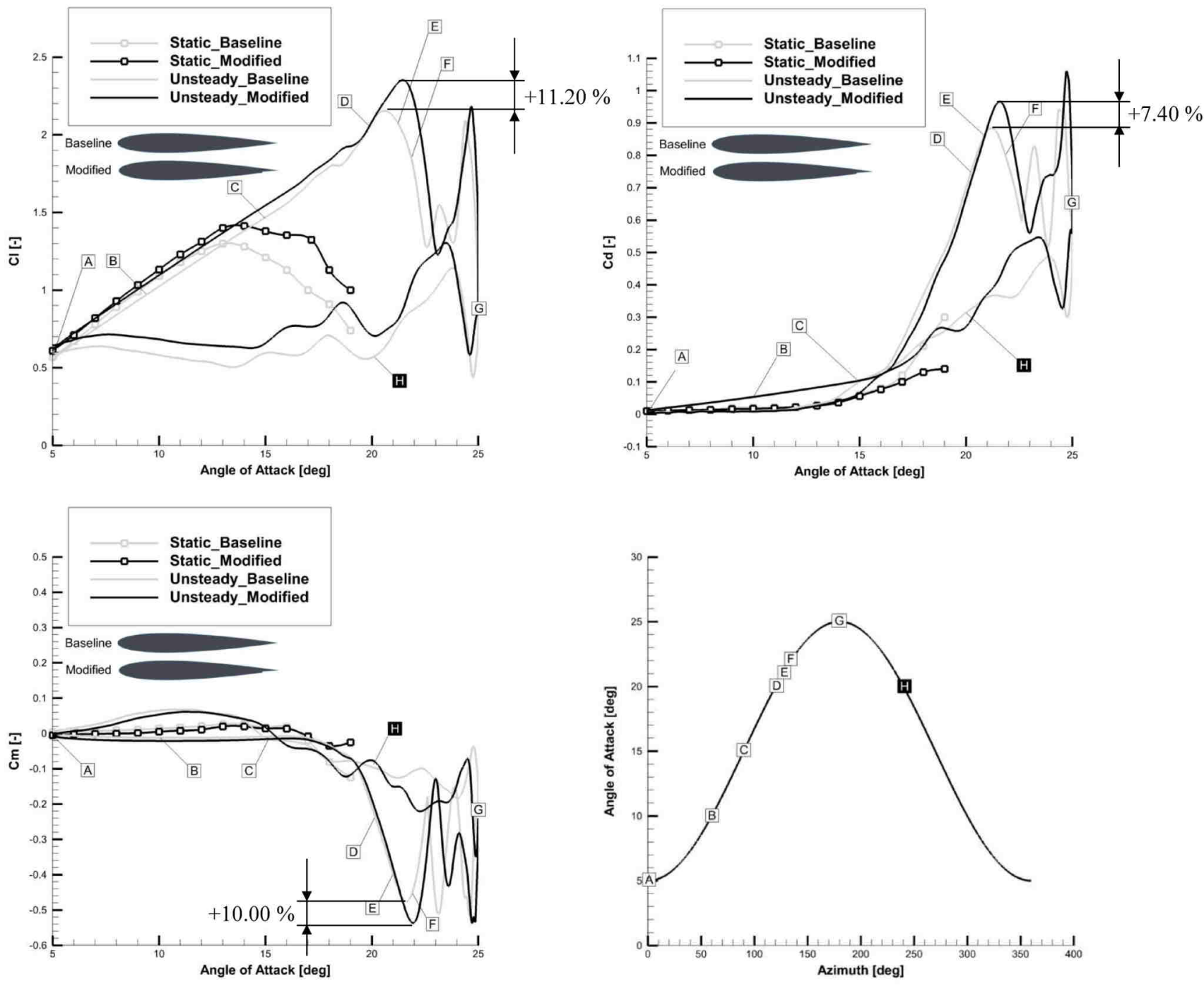

Figure 4.29 Aerodynamic loads for the $\mathrm{G}^{2} \mathrm{~L} 1$ airfoil with the selected azimuthal positions. $M=0.3, \operatorname{Re}=3.76 \times 10^{6}$ and $\alpha=15^{\circ} \pm 10^{\circ}$. 


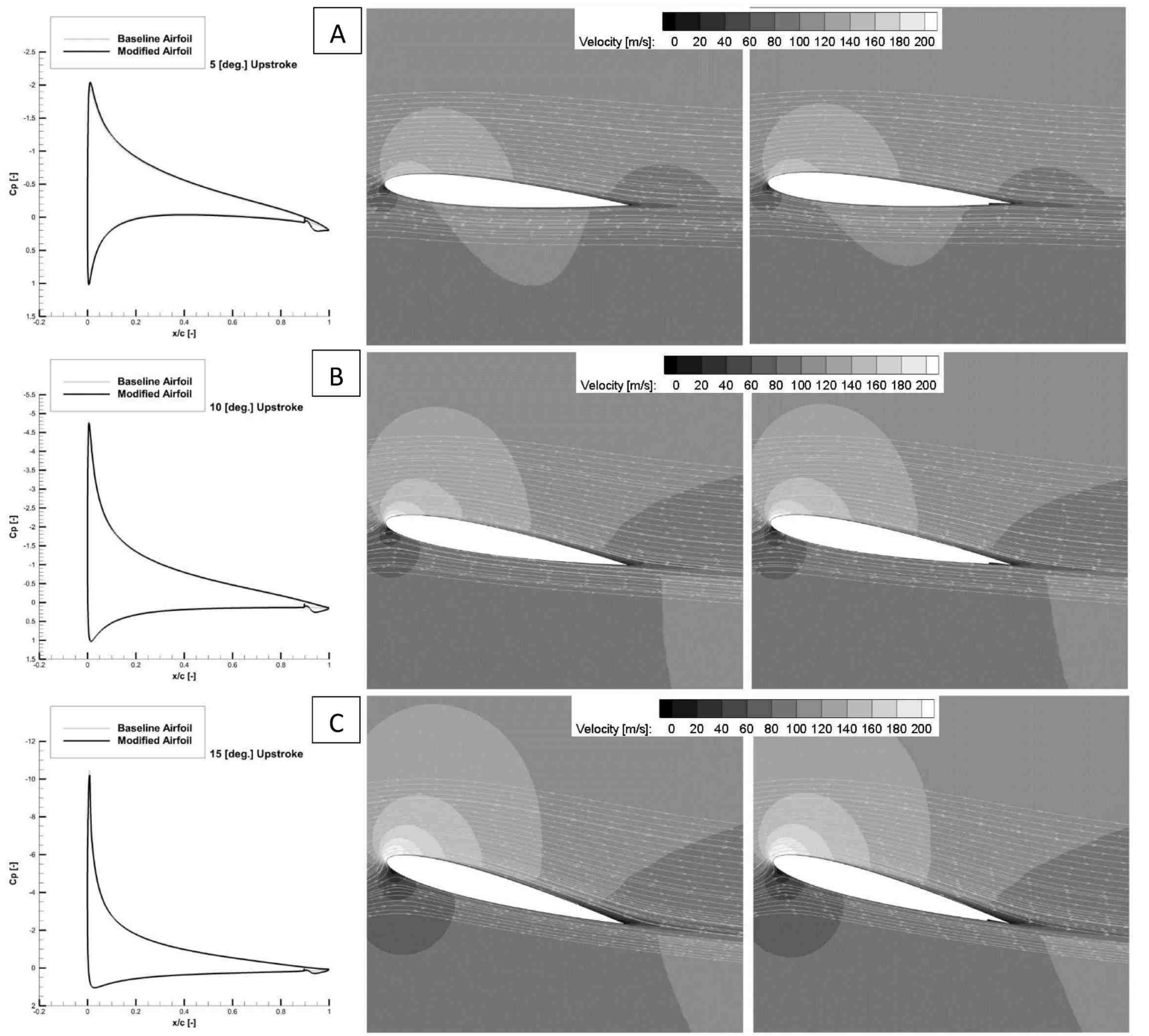

Figure 4.30 Pressure coefficient, instantaneous streamlines and velocity contours for the NACA 0012 airfoil and the $\mathrm{G}^{2} \mathrm{~L} 1$ airfoil. $M=0.3, R e=3.76 \times 10^{6}$ and $\alpha=15^{\circ} \pm 10^{\circ}$. 

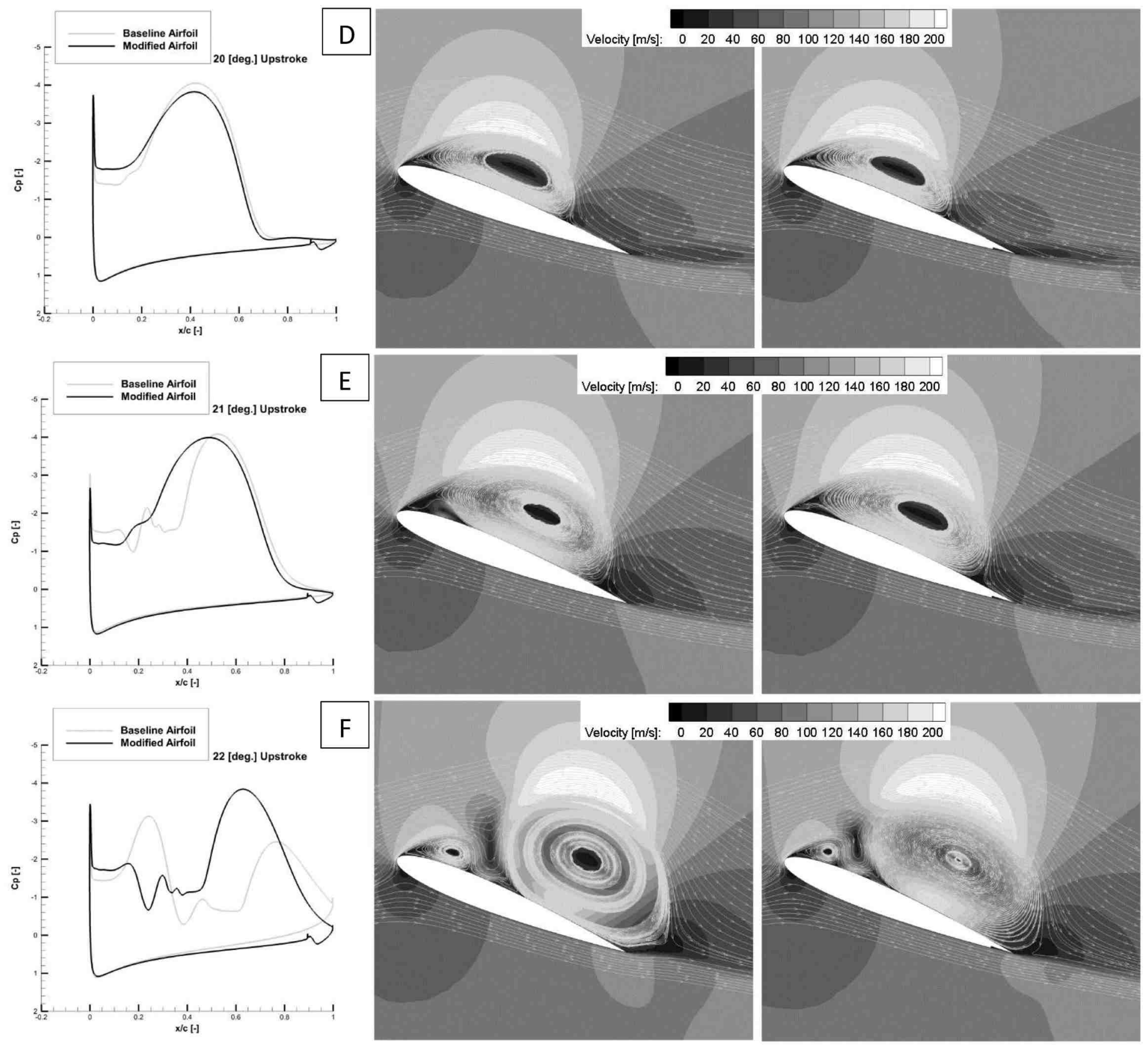

Figure 4.30 (Continued) Pressure coefficient, instantaneous streamlines and velocity contours for the NACA 0012 airfoil and the $\mathrm{G}^{2} \mathrm{~L} 1$ airfoil. $M=0.3, R e=3.76 \times 10^{6}$ and $\alpha$ $=15^{\circ} \pm 10^{\circ}$. 

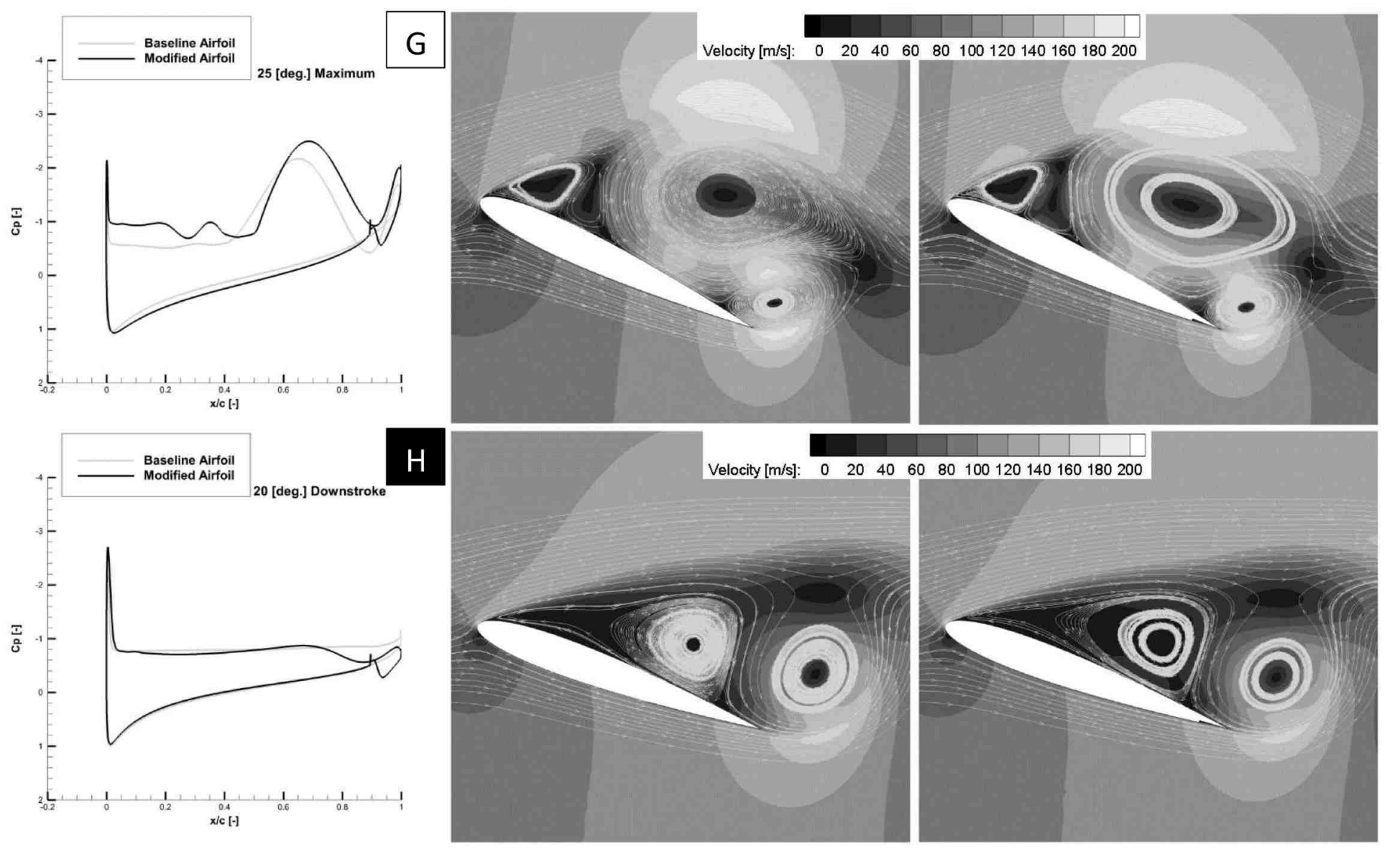

Figure 4.30 (Continued) Pressure coefficient, instantaneous streamlines and velocity contours for the NACA 0012 airfoil and the $\mathrm{G}^{2} \mathrm{~L} 1$ airfoil. $M=0.3, R e=3.76 \times 10^{6}$ and $\alpha$ $=15^{\circ} \pm 10^{\circ}$.

\subsubsection{Fluctuating Freestream Dynamic Stall}

CFD simulations of 2D dynamic stall for an SC1095 airfoil were completed with the numerical parameters described in Chapter 3. Two simulations were conducted. The first one was a dynamic stall case with a compressible constant freestream velocity corresponding to the mean velocity of the fluctuating freestream case. This will serve as the baseline case, to which fluctuating freestream cases will be compared to. The second case is dynamic stall with compressible fluctuating freestream. The discussion of results below is therefore divided into two sections. However, before presenting the results, a 
note needs to be made about the scaling factors used to express the coefficients of aerodynamic forces and moments.

In the current study, for both the constant freestream simulations (CFS) and fluctuating freestream simulations (FFS), lift, drag and pitching moment were scaled by the mean velocity at each instant in time to express the aerodynamic coefficients. This is in contrast to the literature, where forces and moment of FFS cases are scaled by the instantaneous freestream velocity at each instant in time, for example, Gosselin et al. [60], Kerho [71], Hird et al. [77, 78], Zhao et al. [79] and Gregory et al. [106]. This type of scaling leads to fluctuating freestream (FFS) results to be higher than those under constant freestream (CFS), as illustrated for example in Fig. 4.31, which reproduces the experimental results of [106]. However, this gives a false impression about how the FFS and CFS loads relate to each other in reality, i.e. in dimensional terms. Fig. 4.32 illustrates that the actual loads, i.e. the dimensional forms of the aerodynamic forces and moment, behave in the opposite manner and that for these the FFS values are actually smaller than the CFS values (Fig.4.32). The study dictates the believe that the nondimensionalized aerodynamic loads as well their dimensional equivalents should show the same trends. Therefore, it is proposed in this thesis that the aerodynamic loads are scaled by the mean velocity and density, which yield the correct trends between the FFS and CFS results (see Fig. 4.32). 

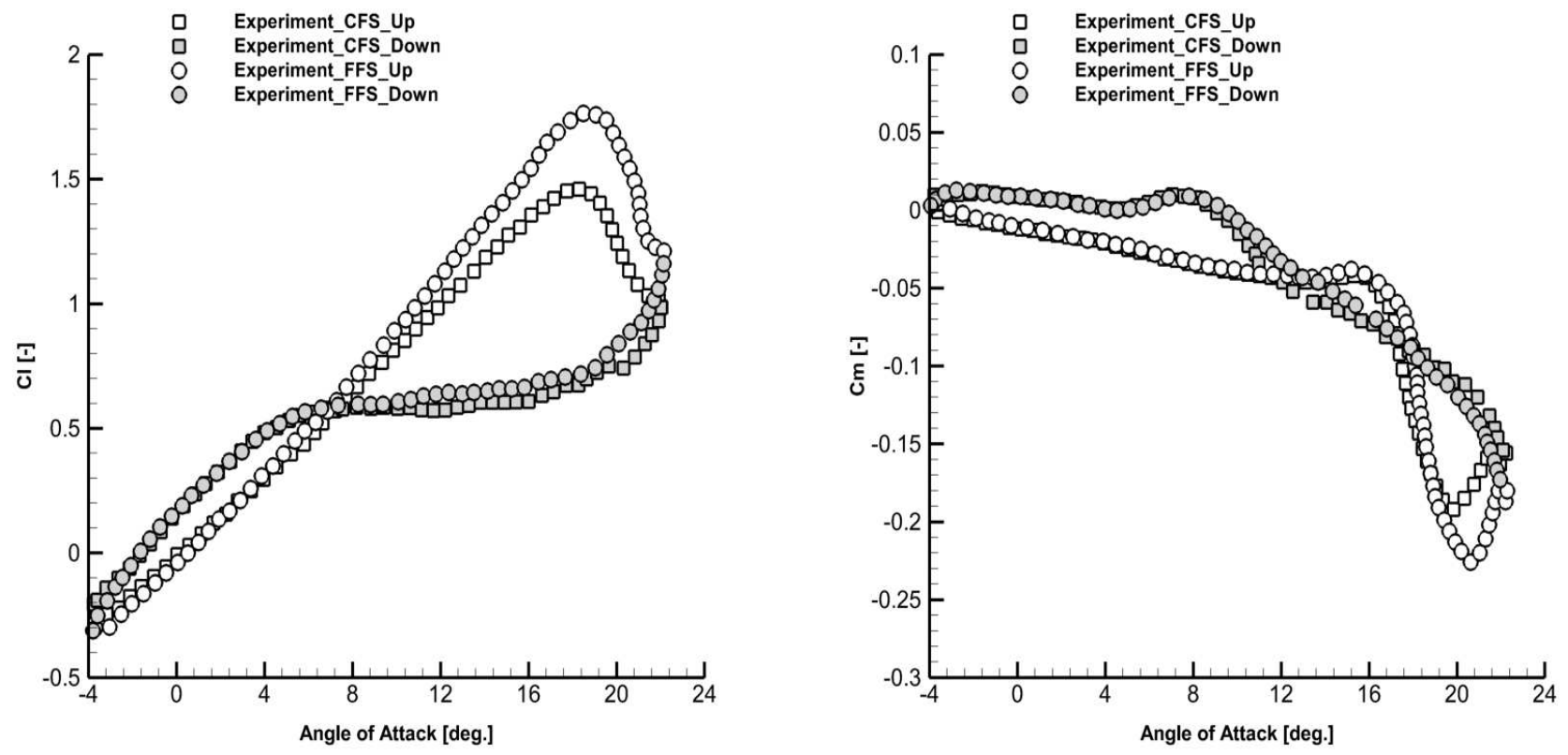

Figure 4.31 CFS $(M=0.4)$ vs. FFS $(M=0.4 \pm 0.08)$ dynamic stall of [106] experiment.

SSC-A09 airfoil, $R e=3 \times 10^{6}, k=0.05, \alpha=8.5^{\circ} \pm 13^{\circ}$.

A detailed flow visualization of the same case as in [106], at angle of attack $15.14^{\circ}$ is also provided in Fig. 4.33 to support the fact above. One can clearly see the increased size of separation in FFS compared to that of the CFS, and hence, lift should be smaller in the FFS case compared to the CFS case, not the opposite.

This is further proof that the recommended scaling, based on the mean values appears to be correct. This was the method used in the current study. 

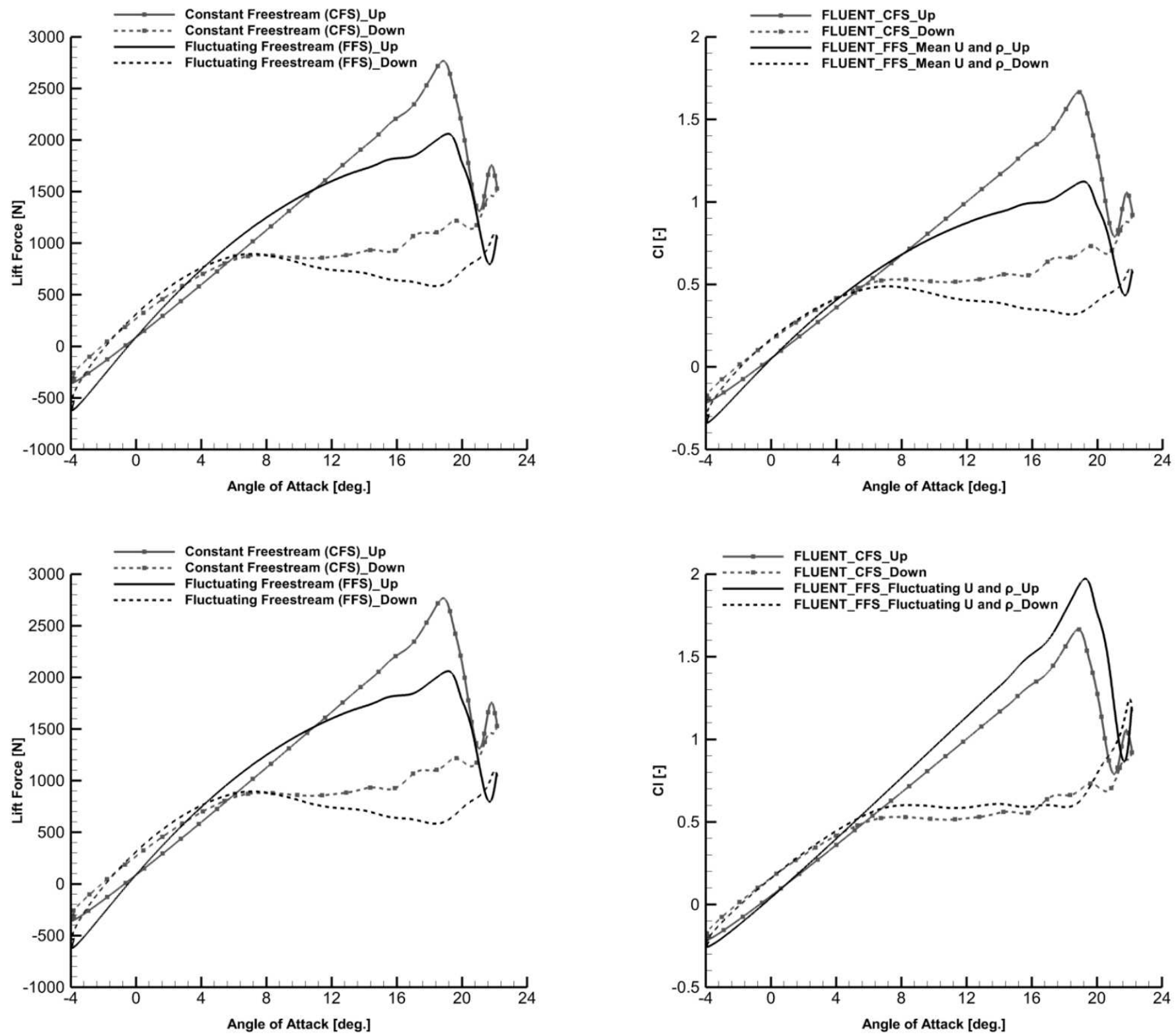

Figure 4.32 CFS $(M=0.4)$ vs. FFS $(M=0.4 \pm 0.08)$ dynamic stall. Loads vs. coefficients.

Scaled using the mean values vs. fluctuating values. Case study of [106] experiment.

$$
\mathrm{SSC}-\mathrm{A} 09, \operatorname{Re}=3 \times 10^{6}, k=0.05, \alpha=8.5 \pm 13 \text {. }
$$



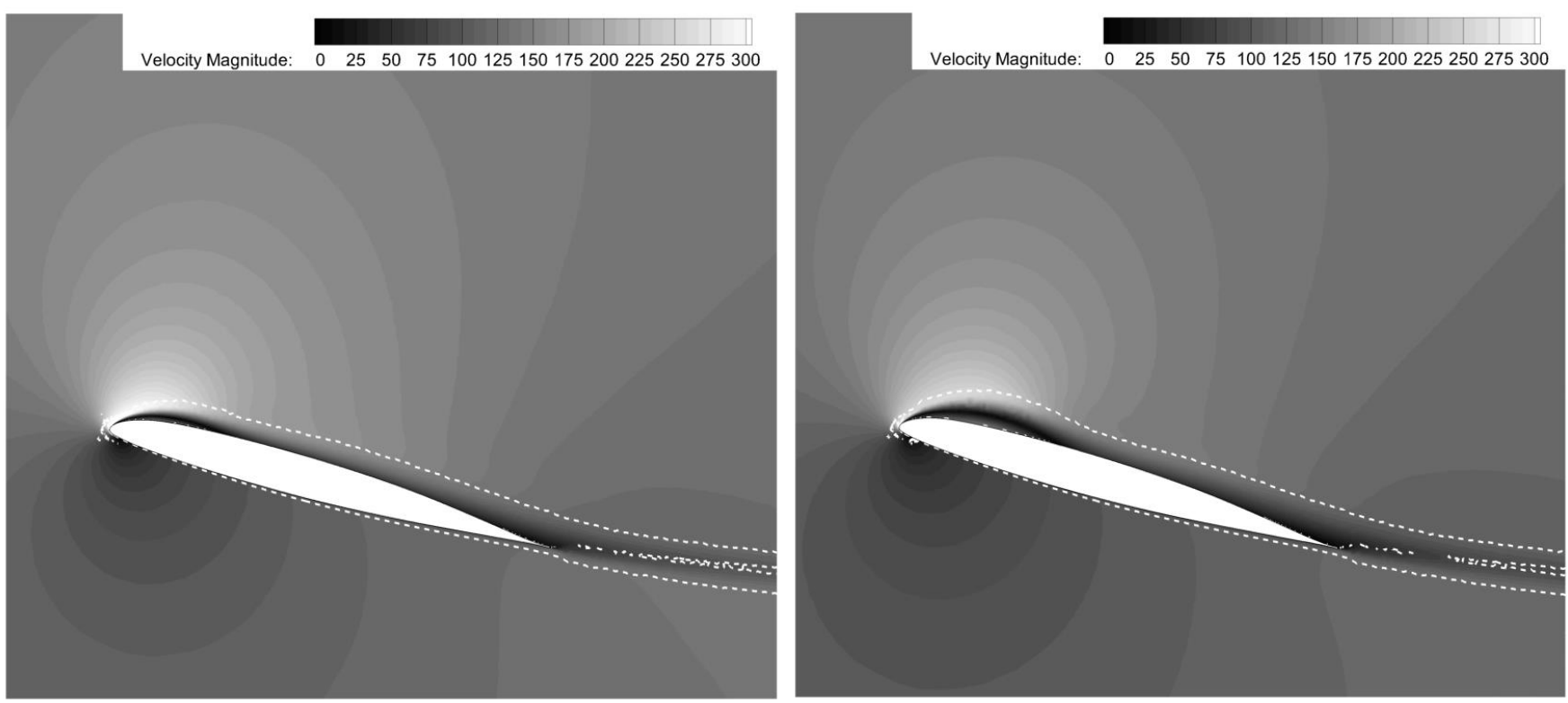

Figure 4.33 CFS (left) vs. FFS (right) dynamic stall. White dashed-line represents maximum vorticity. Case study of [106] experiment. SSC-A09 airfoil, $R e=3 \times 10^{6}$,

$$
k=0.05, \alpha=15.14^{\circ} \text { and } M=0.4 \pm 0.08 \text {. }
$$

Compressible Constant Freestream Dynamic Stall: UH-60A case

For comparing the CFS and FFS dynamic stall results, as it is intended in this study, one must choose a common mean freestream Mach number for both cases. For this, a common freestream Mach number was selected by taking the rotational velocity at $86.5 \%$ radius of the blade, which according to Table 3.2, in Chapter 3, corresponds to Mach 0.537. Therefore, this will be the freestream for the constant freestream dynamic stall simulation as well as the mean value used for non-dimensionalizing the aerodynamic loads in the fluctuating freestream cases too. According to Table 3.2, in Chapter 3, the amplitude of the freestream fluctuation will be Mach 0.205, yielding a fluctuating freestream of $0.537 \pm 0.205$. 
Results for the CFS simulations are shown in Fig. 4.34. Note that the main influence of exposing an airfoil to dynamic stall conditions (in terms of frequency and angle of attack fluctuation) at the constant freestream of Mach 0.537 leads to the domination of compressibility effects. This is best manifested by the dramatic decrease of the stall angle of attack from 20 degrees (as was seen at Mach 0.4 in Fig. 4.31) to only about 12 degrees, i.e. lift stall occurs closer to the static stall value, rather than at the usual overshoot associated with a dynamic stall vortex. This is due to the occurrence of transonic flow and shock-induced boundary layer separation on the upper surface well below the "classical" dynamic stall angle of attack. This means that compressibility effects dominate the stall mechanism, a fact supported also in the literature, for example in [109] and [110].
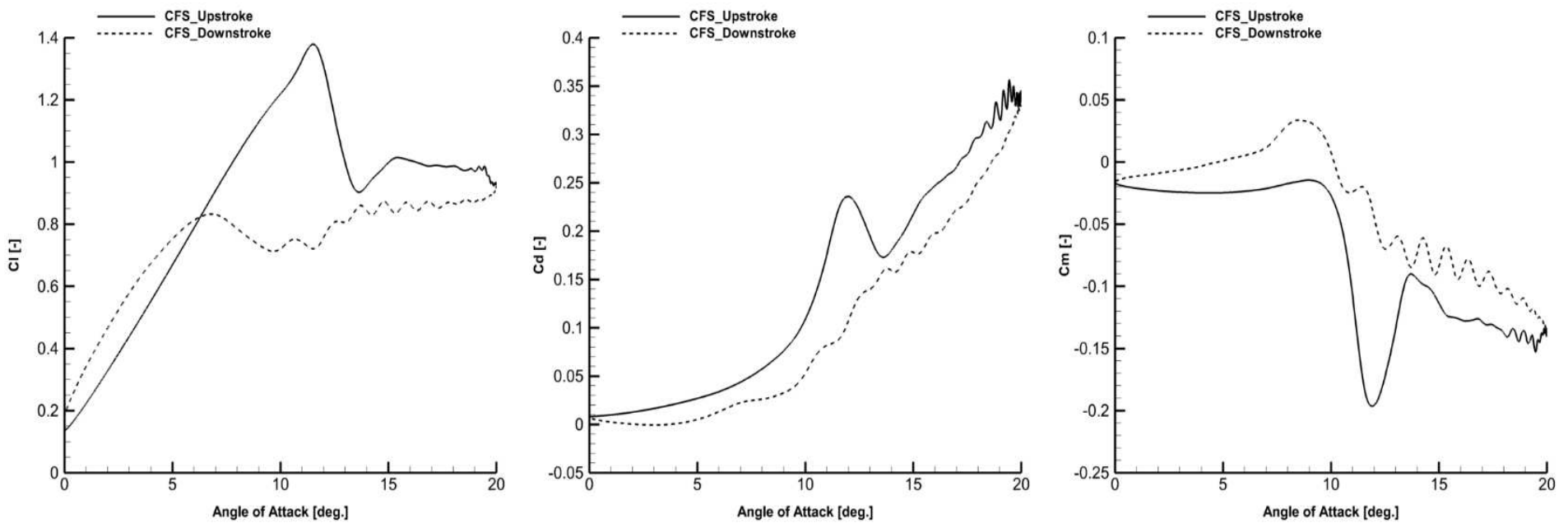

Figure 4.34 Compressible constant freestream (CFS) dynamic stall. SC1095, $R e=$ $6.1 \times 10^{6}, f=4.25 \mathrm{~Hz}, \alpha=10 \pm 10$ and $M=0.537$. 


\section{Fluctuating Freestream Dynamic Stall: UH-60A case}

The FFS simulation conditions of the main rotor blade of UH-60A in a forward flight are sketched in Fig. 4.35. The selected $r / R=0.865$ section is represented by the dashed-arrows in the velocity distribution plot. Because of the fluctuating relative freestream of $0.537 \pm 0.205$, the advancing blade will be subject to transonic flow ( $M=$ $0.742)$ while the retreating blade to dynamic stall $(M=0.332)$. A detailed analysis of the flow via flow visualizations is provided in Fig. 4.36 and according to the selected frames (boxed letters) shown in Fig. 4.36.

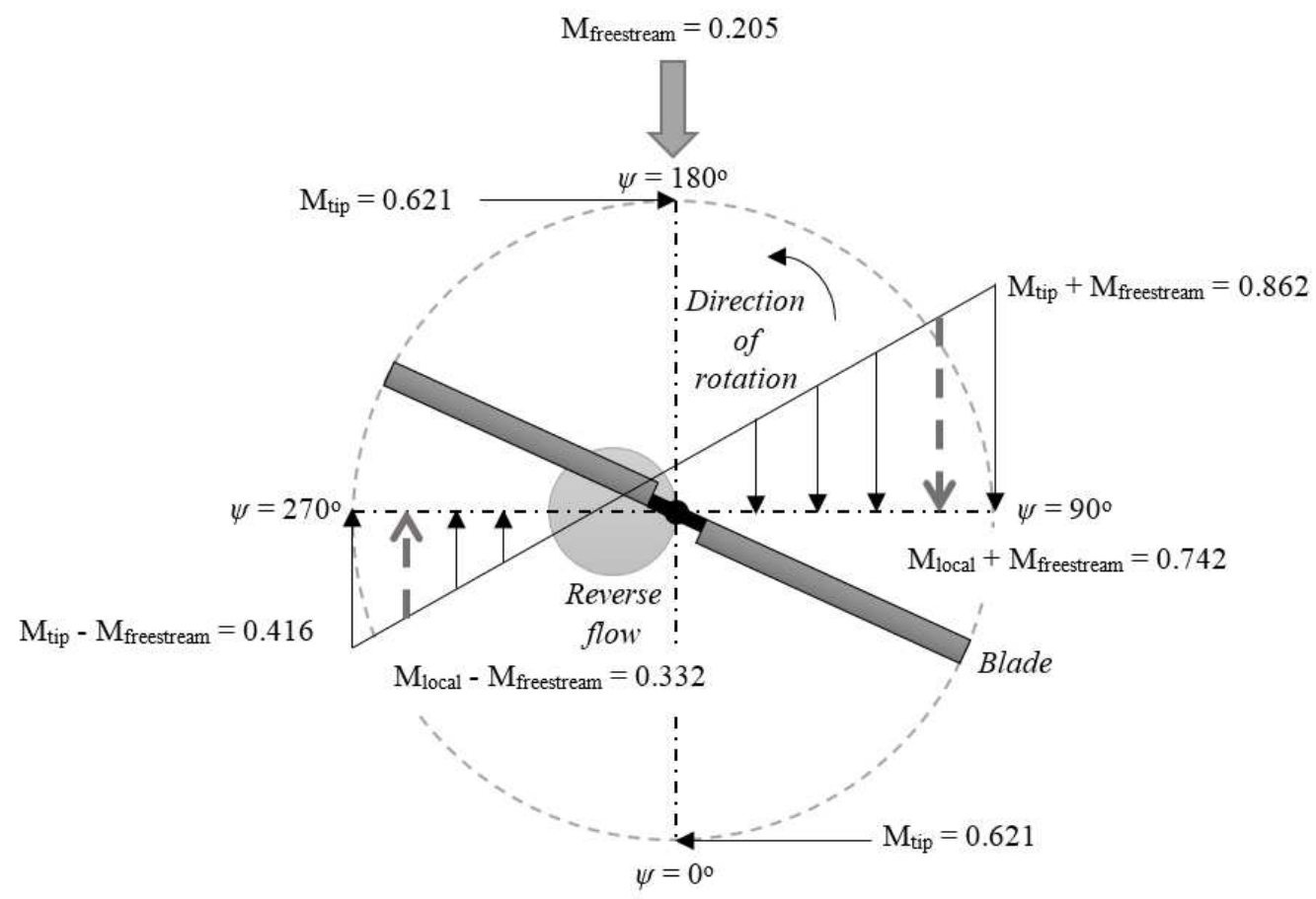

Figure 4.35 Top view sketch of UH-60A main rotor disk showing Mach number distribution along the blade in a forward flight phase according to Table 3.2, in Chapter 3. 

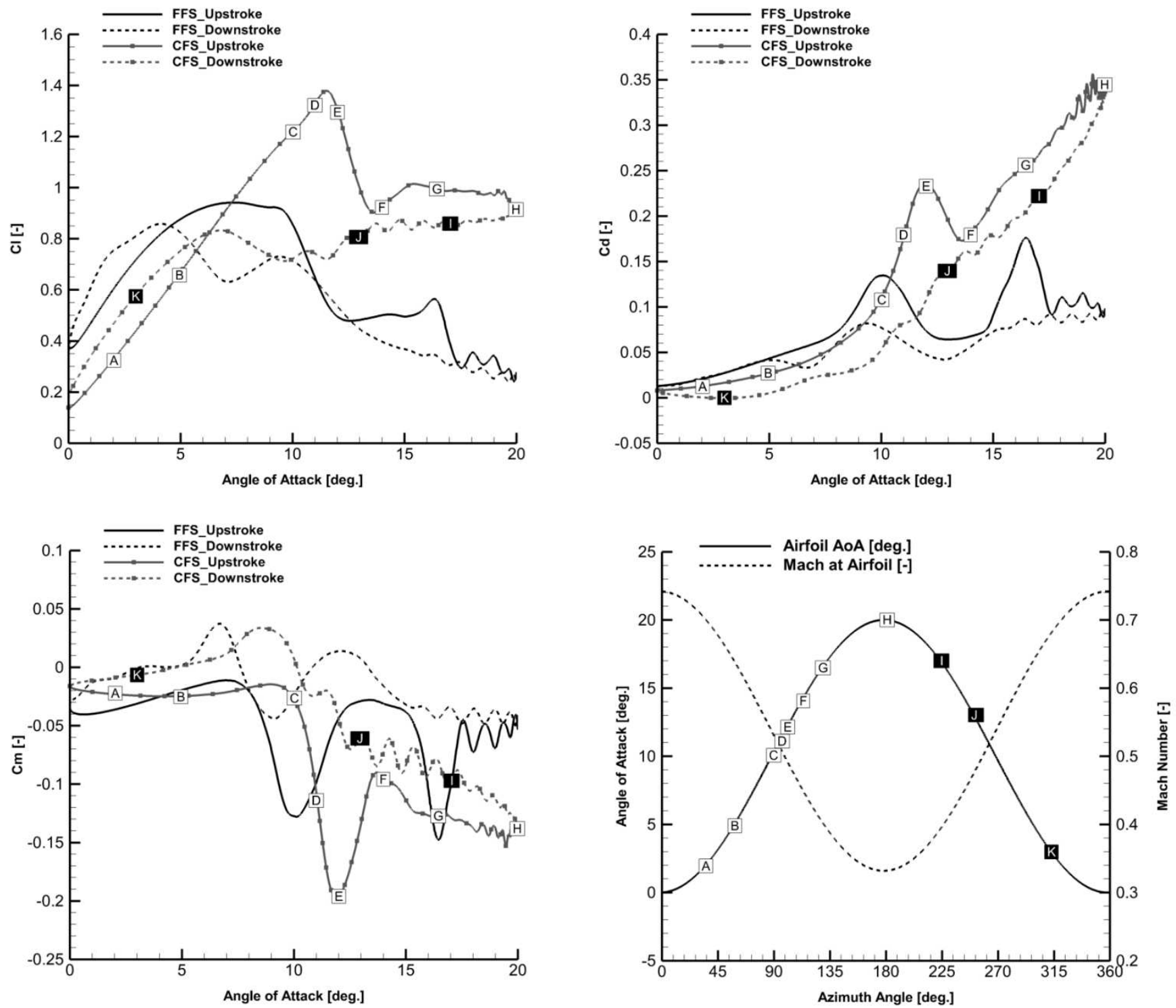

Figure 4.36 Comparison of aerodynamic loads at Fluctuating Freestream (FFS) dynamic stall, $(M=0.537 \pm 0.205)$ and at Constant Freestream (CFS) dynamic stall $(M=0.537)$ with the selected azimuthal positions. SC1095, $R e=6.1 \times 10^{6}, f=4.25 \mathrm{~Hz}$, and $\alpha=$

$$
10^{\circ} \pm 10^{\circ}
$$

From Fig. 4.36 above, there are four substantial differences between the "real life" dynamic stall at FFS and the "representative dynamic stall" at CFS: 
a) Stall in the aerodynamic loads in the FFS case occur earlier than in the CFS case, i.e. at lower angles of attack.

b) The peak values at stall are smaller in the FFS case than in the CFS case.

c) Before stall, the aerodynamic loads are generally larger in the FFS case than in the CFS case.

d) Beyond stall, the aerodynamic loads are generally lower in the FFS case than in the CFS case.

Moreover, due to the compressibility effects in the FFS cases, there are two large peaks during the upstroke phase (Fig. 4.36). The presence of these can be explained by examining the flow visualisation frames on Fig. 4.37. From these it can be seen that the first peak (Frame C) is generated by the shock-formation as the angle of attack increases, while the second one likely because of the stationary shockwave at the leading-edge causing shock wave - boundary layer interaction and a consequent vortex shedding, which forms alternating leading and trailing-edges vortices (shock-induced dynamic stall). This process is visible from Fig. 4.36, Frames C, D, E, F and G.

In the FFS case, there is a large shock wave at the quarter-chord position of the airfoil, starting from the beginning of the upstroke phase (for example, see Frame A, Fig. 4.36 at $\alpha=2^{\circ}$ and $\psi=36^{\circ}$ ). This diminishes in strength and moves upstream along the airfoil as the angle of attack increases, until the first leading-edge vortex is shed (Frame C, Fig. 4.37 at $\alpha=10^{\circ}$ and $\psi=90^{\circ}$ ). During this process, the flow upstream and downstream of the shock appears to be attached.

The lift during upstroke phase was higher compared to the CFS case from angle of attack $0^{\circ}$ to $7^{\circ}$. This is because of the higher speed on the upper surface of the airfoil during this range. However, lift starts to decrease during the next upstroke phase due to 
the decrease in speed on the airfoil upper surface. This is true because this will lead to higher pressure on the upper surface compared to that on the lower surface. This can be illustrated by Frame $\mathrm{C}$ on Fig. 4.37, where the shockwave is moving upstream during the upstroke phase. The separated flow region starts to increase and becomes large enough to cause the lift coefficient for the FFS case to be less than that for the CFS case, which is logical since separated flow generates less lift than attached flow. This further supports the argument, that the aerodynamic loads shall be non-dimensionalized by the mean velocity and not the instantaneous velocity to reflect the flow physics.

On the other hand, because of the shockwave was present from the beginning of the upstroke phase, between angles of attack $0^{\circ}$ to $10^{\circ}$ the drag was higher in the FFS case compared to that in the CFS case (Fig. 4.37). For the rest of the upstroke, the drag in the FFS case becomes lower than that in CFS case. This is likely due to the shockwave moving upstream. As a result, the wave-drag did not have enough time to develop into a value that could affect the total drag. Moreover, the instantaneous velocity starts to decrease after Frame $\mathrm{C}$ (Mach number decreases) and this leads to a decrease in the speed, and hence, the drag.

Regarding the FFS pitching moment, at angle of attack $12^{\circ}$ (Frame E in Fig. 4.37), the pitching moment was remarkably lower in the FFS case than in the CFS case. This behaviour is due to the trailing-edge vortex, which has the effect of decreasing the pitching moment as well as the drag.

It is noteworthy that in the FFS case, when the angle of attack increases, the leading-edge vortex convected aft to the trailing-edge is shrunk in size and appears to be closer to the airfoil. On the other hand, the trailing-edge vortex starts stronger at angle of attack $12^{\circ}$ and becomes weaker as the freestream Mach number decreases during the 
upstroke phase (see Frames E to H, Fig. 4.37). Further in the upstroke phase of the FFS case, at angle of attack $20^{\circ}$ (Frame H, Fig. 4.37), the wake of the airfoil features a weaker trailing-edge vortex (divided into two vortices) with a leading-edge vortex significantly lower in its strength compared to that in the CFS case.

The downstroke phase begins with a leading-edge vortex shedding. As the vortex is shed downstream over the airfoil, a significant enhancement in lift and a reduction in the magnitude of the pitching moment can be observed due to the increasing freestream Mach number and the flow re-attachment process. For example, from Fig. 4.36 Frame I, the pitching moment is higher due to these reasons, and as a result, the drag is significantly reduced compared to that in the CFS case. Also, in Fig. 4.36 Frame K, although the drag has increased, lift has greatly increased compared to that in CFS case. This is due to the presence of the shockwave that is clearly shown in Fig. 4.37, Frame K, which it was absence in the CFS case. 

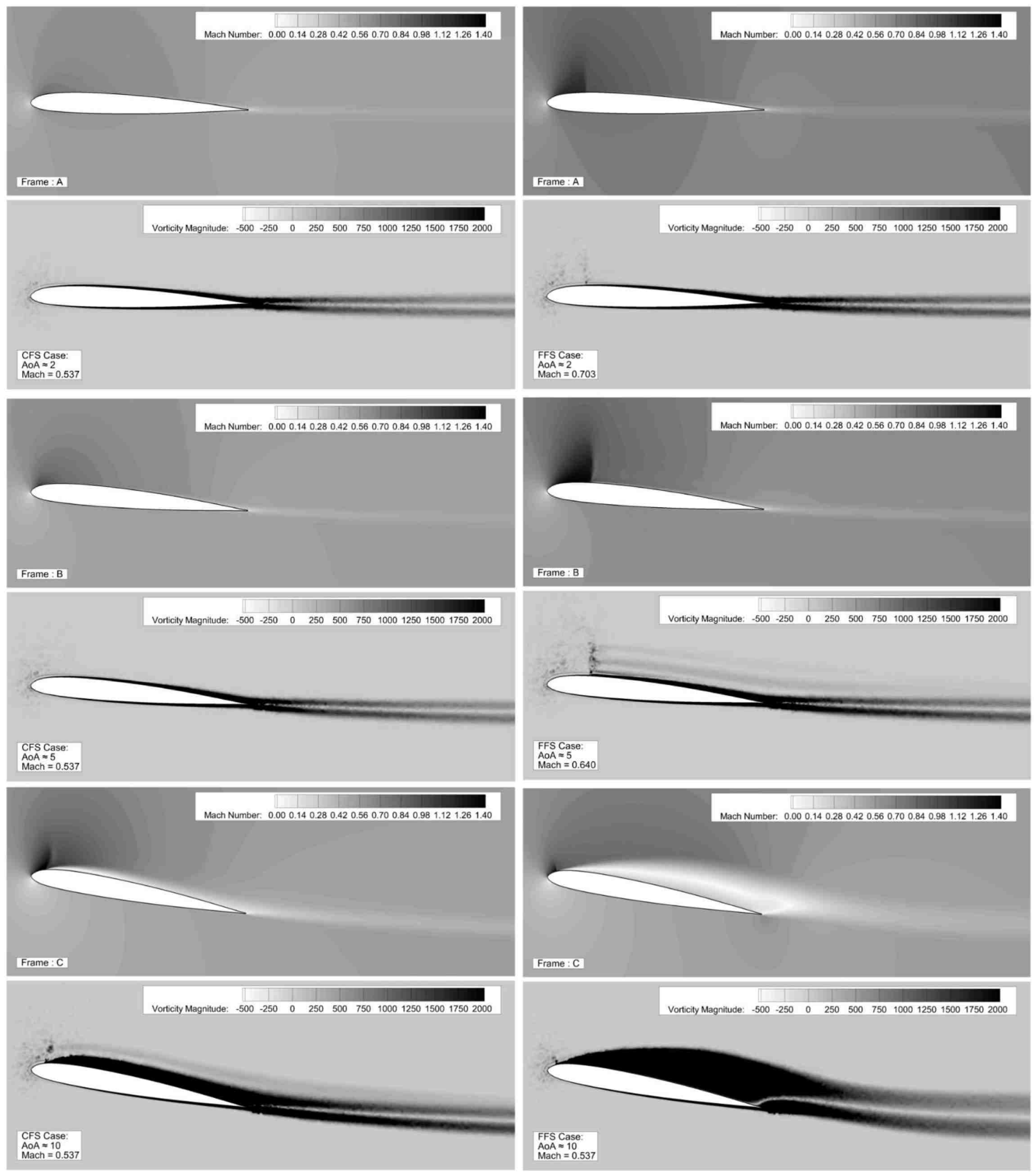

Figure 4.37 Mach contours and instantaneous vorticities. SC1095 airfoil, $M=0.537$ (left) and $0.537 \pm 0.205$ (right), $R e=6.1 \times 10^{6}, f=4.25 \mathrm{~Hz}$ and $\alpha=10^{\circ} \pm 10^{\circ}$. 

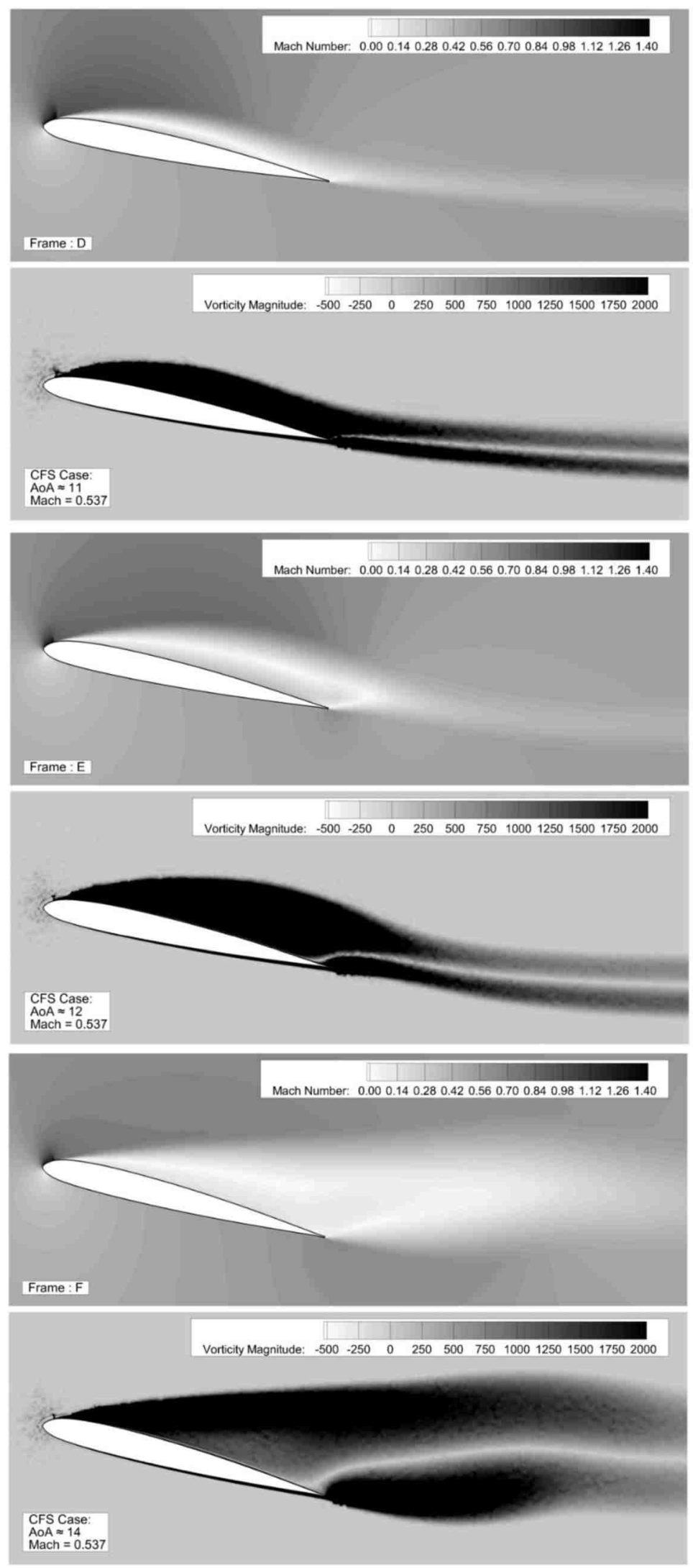
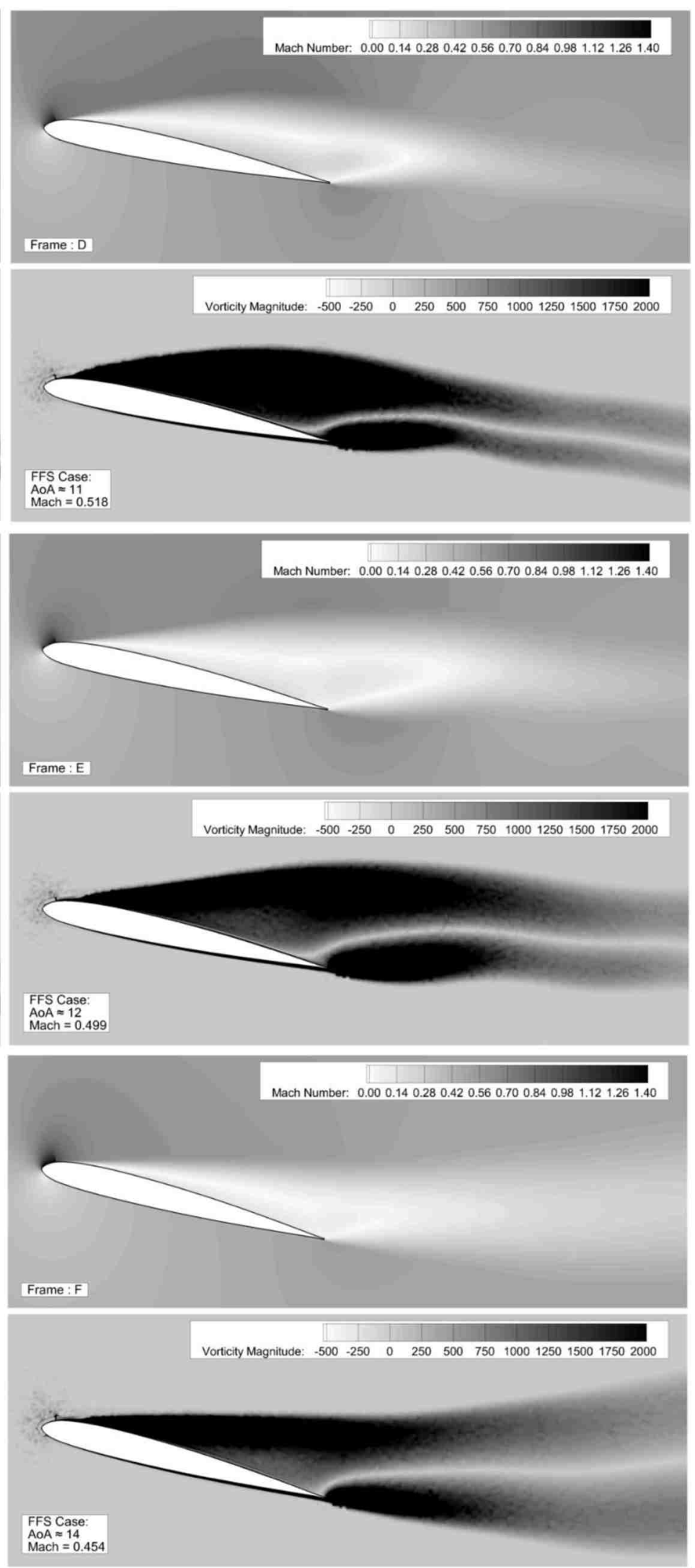

Figure 4.37 (Continued) Mach contours and instantaneous vorticities. SC1095 airfoil, $M=0.537$ (left) and $0.537 \pm 0.205$ (right), $R e=6.1 \times 10^{6}, f=4.25 \mathrm{~Hz}$ and $\alpha=10^{\circ} \pm 10^{\circ}$. 

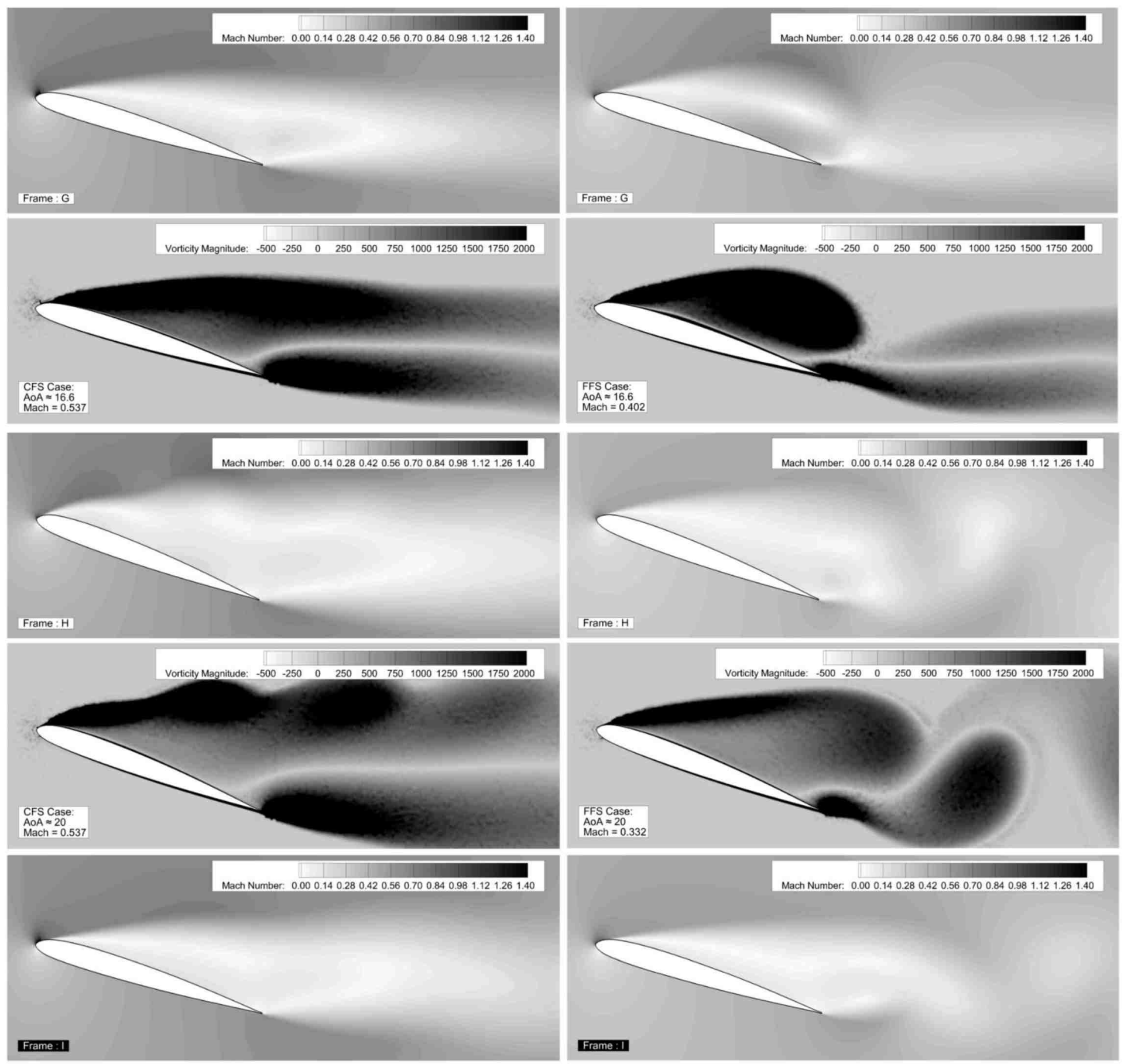

Frame:1

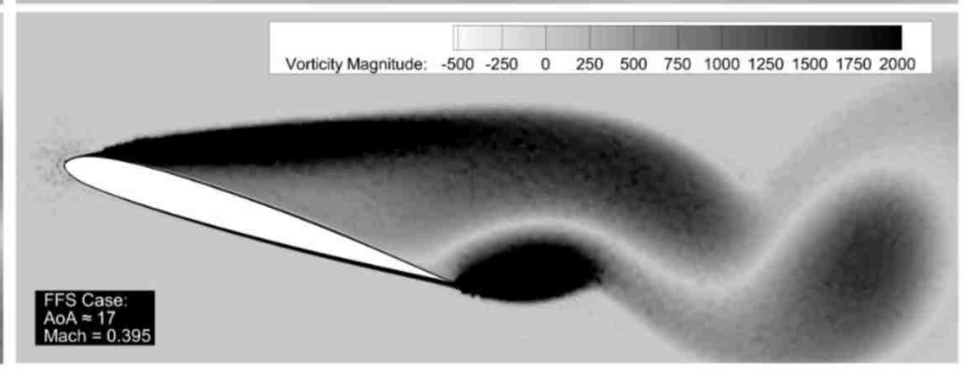

Figure 4.37 (Continued) Mach contours and instantaneous vorticities. SC1095 airfoil, $M=0.537$ (left) and $0.537 \pm 0.205$ (right), $R e=6.1 \times 10^{6}, f=4.25 \mathrm{~Hz}$ and $\alpha=10^{\circ} \pm 10^{\circ}$. 

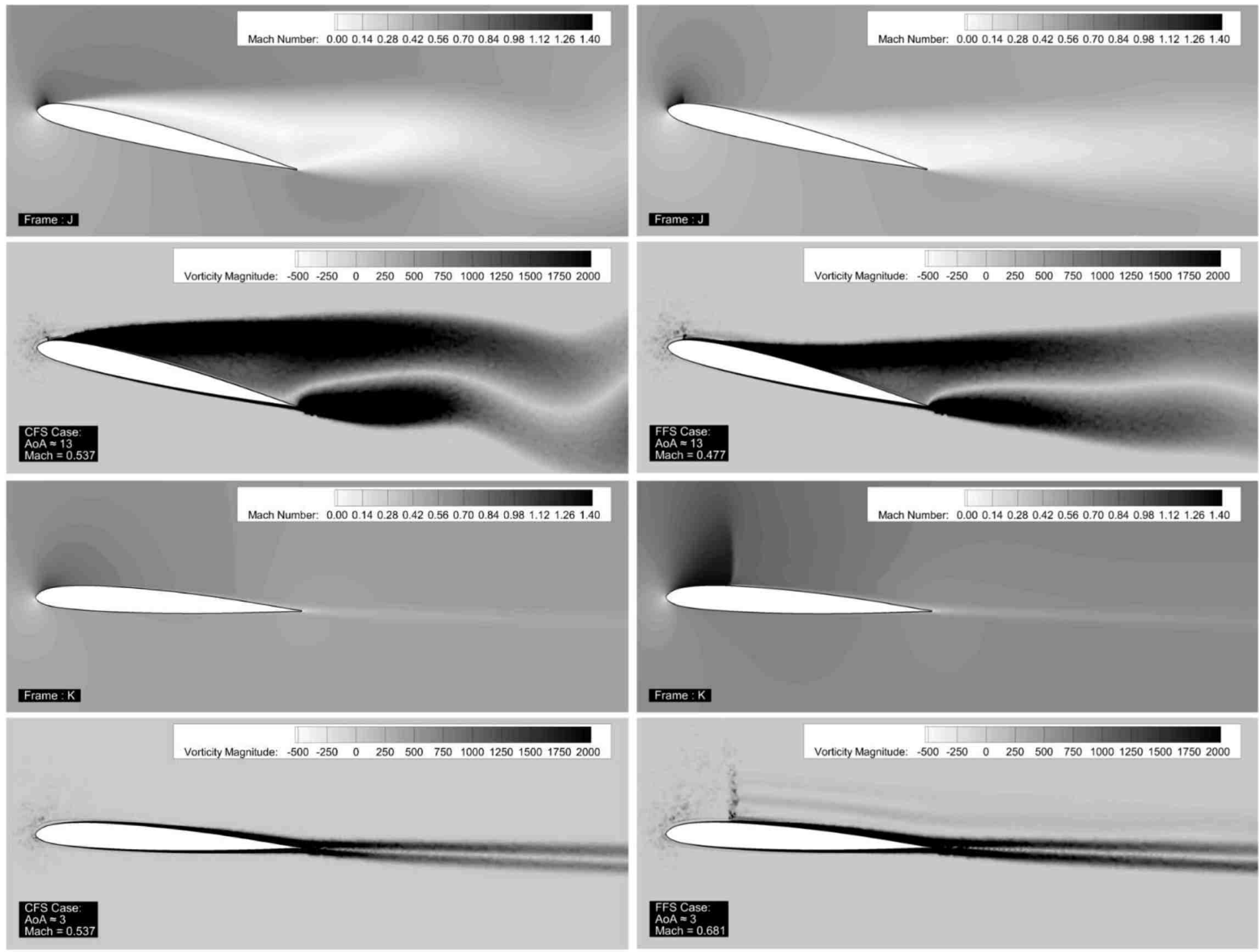

Figure 4.37 (Continued) Mach contours and instantaneous vorticities. SC1095 airfoil, $M=0.537$

(left) and $0.537 \pm 0.205$ (right), $R e=6.1 \times 10^{6}, f=4.25 \mathrm{~Hz}$ and $\alpha=10^{\circ} \pm 10^{\circ}$. 


\subsubsection{Fluctuating Freestream Dynamic Stall Control}

The current study imitates the flight conditions, which a UH-60A main rotor blade section at $r / R=0.865$ would experience in forward flight at Mach number of 0.205. The UH-60A main rotor blade consists of two airfoils, SC1095 and SC1094 R8 [111]. At $r / R=0.865$, the airfoil is the SC1095 (Fig. 4.38), hence, this was selected as the baseline airfoil for this section.

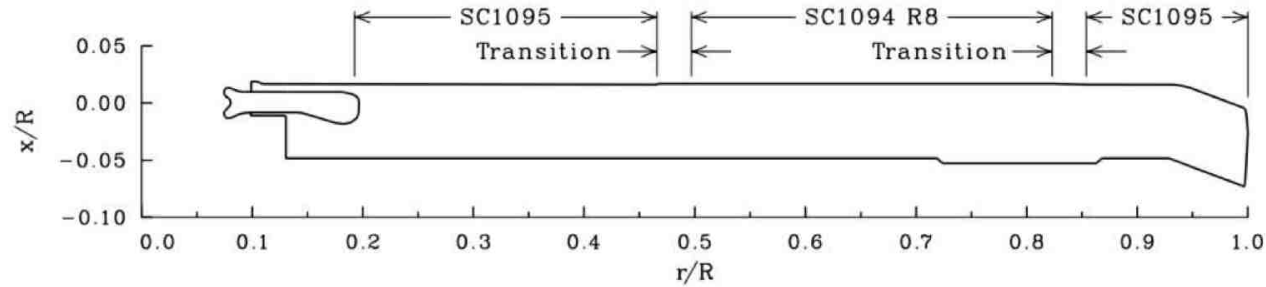

Figure 4.38 UH-60A main rotor blade and SC1095 airfoil [111].

Twenty surface-based passive flow controls, of those depicted in Fig. 4.2, and with the geometric specifications found in Table 4.1 was implemented on the SC1095 airfoil by modifying one of the airfoil surfaces and by introducing a Surface-Based Trapped Vortex Generator (SGVT) in it. For the sake of comparison, the geometries used in this section are similar to those of Section 4.4.1 of constant freestream dynamic stall. However, the stepped modifications $\left(1^{\text {st }}\right.$ generation) were excluded from the current study due to their poor performance in transonic flow, as previously established in this chapter. 
To further increase the confidence in the proposed modifications, the same geometries chosen for Section 4.4.1 for constant freestream $(\mathrm{M}=0.3)$ dynamic stall over NACA 0012 airfoil, will be investigated here using the transonic airfoil SC1095 (real-life blade airfoil). Fig. 4.39 illustrates SC1095 transonic airfoil with the selected modification implement on the airfoil surface.

Figure 4.39 SC1095 transonic airfoil with the selected modifications applied.

As mentioned before, unlike the typical, constant freestream dynamic stall, compressible dynamic stall under fluctuating freestream have two phases, transonic phase and dynamic stall phase. Hence, several parameters were chosen to assess the performance of the airfoil with modification. These parameters were: a) the peaks of the lift-to-drag ratio, drag, and pitching moment coefficients $\left(C_{l} / C_{d}, C_{d}, C_{m}\right)$, for both the transonic and dynamic stall phases, and b) the average values of the aerodynamic coefficients $\left(C_{l} / C_{d}, C_{d}\right.$, and $\left.C_{m}\right)$ over one rotor cycle. The latter choice was made because the current 2D study - naturally - disregards the "3D effects" of the helicopter main rotor blade aerodynamics, i.e., crossflow, Coriolis forces and tip vortices. It is believed that the averaged values of the aerodynamic coefficients over one rotor cycle provide a decent evaluation of the aerodynamic performance and loads of the blade section. Thus, the average values over one rotor cycle will also be used to evaluate the performance of the proposed passive flow control techniques. 
The objective of the present work was to design a passive flow control concept, which can serve as a viable alternative to active flow control techniques, since due to its simplicity it should have the promise of easier implementation in practice. The intended passive flow control technique should not deteriorate the lift characteristics of the original airfoil significantly, while providing noteworthy improvements in drag and pitching moment reductions, i.e. it should decrease peak drag and peak negative pitching moments. Reducing the pitching moment is desired since this exhibits as vibrations in the pitch links during forward flight.

All simulations completed with the numerical parameters described in Chapter 3, Section 3.5 , for the UH-60A test case at 0.865 blade radius, i.e. $f=4.25 \mathrm{~Hz}, R e=$ $6.1 \times 10^{6}, M=0.537 \pm 0.205$ and $\alpha=10^{\circ} \pm 10^{\circ}$.

It can be seen from Table 4.4, that the SC1095 airfoils with the flow control concepts out-perform the clean (baseline) airfoil, in fact, most of the designs perform remarkably well. For instance, virtually all surface modifications reduced the peak negative pitching moment and peak drag for the complete cycle, and by as much as $23 \%$ and $17 \%$, respectively, for the transonic phase, and by $71 \%$ and $53 \%$, respectively, for the dynamic stall phase. A penalty in the peak lift-to-drag ratio occurred only in the transonic phase, between angles of attack $0^{\circ}$ and $2^{\circ}$. Beyond this, the modified airfoil dominates the baseline airfoil as it will be seen later. Moreover, the penalty is very small and its duration within one revolution is short too.

The averaged results over one rotor cycle confirms the potentials of the proposed passive flow control technique to enhance the performance of the helicopter main rotor as can be seen from Table 4.4 . 
The flow physics behind these results were explained by the detailed analysis of the flow by means of flow visualization comparisons between the baseline airfoil and the modified airfoil. However, due to space constraints, only 2 airfoils were analyzed, 1 with upper surface and 1 with lower surface modifications. The airfoils, for which detailed analysis is provided are indicated by the gray arrows in Table 4.4.

Table 4.4 Summary of the CFD simulation results for the SC1095 at $f=4.25 \mathrm{~Hz}, R e=$

$$
6.1 \times 10^{6}, M=0.537 \pm 0.205 \text { and } \alpha=10^{\circ} \pm 10^{\circ} \text {. }
$$

\begin{tabular}{|c|c|c|c|c|c|c|c|c|c|}
\hline \multicolumn{10}{|c|}{ Upper Surface Modifications } \\
\hline \multicolumn{10}{|c|}{$2^{\text {nd }}$ Generation: Trailing-vortex Modifications } \\
\hline & \multicolumn{3}{|c|}{ Transonic Phase \% $\Delta$} & \multicolumn{3}{|c|}{ Dynamic Stall Phase \% $\Delta$} & \multicolumn{3}{|c|}{ Average of One Rotor Cycle \% $\Delta$} \\
\hline Model & $\begin{array}{l}C_{l} / C_{d}[-] \\
\quad \operatorname{Max}\end{array}$ & $C_{d}[-] \mathrm{Max}$ & $C_{m}[-]$ Max & $\begin{array}{l}C_{l} / C_{d}[-] \\
\quad \operatorname{Max}\end{array}$ & $C_{d}[-]$ Max & $C_{m}[-]$ Max & $\begin{array}{l}C_{l} / C_{d}[-] \\
\text { Ave. }\end{array}$ & $C_{d}[-]$ Ave. & $C_{m}[-]$ Ave. \\
\hline $\mathrm{G}^{2} \mathrm{U} 1$ & 9.58 & -10.60 & -12.51 & 8.31 & -28.30 & -42.20 & 14.00 & -14.69 & -31.44 \\
\hline $\mathrm{G}^{2} \mathrm{U} 2$ & 4.43 & -8.14 & -11.56 & 12.54 & -43.44 & -59.90 & 9.72 & -19.00 & -47.67 \\
\hline $\mathrm{G}^{2} \mathrm{U} 3$ & 6.71 & -9.42 & -13.45 & 12.10 & -44.20 & -61.00 & 10.76 & -19.12 & -41.36 \\
\hline \multicolumn{10}{|c|}{$3^{\text {rd }}$ Generation: Body-trapped vortex Modifications } \\
\hline & \multicolumn{3}{|c|}{ Transonic Phase \% $\Delta$} & \multicolumn{3}{|c|}{ Dynamic Stall Phase \% $\Delta$} & \multicolumn{3}{|c|}{ Average of One Rotor Cycle \% $\Delta$} \\
\hline Model & $\begin{array}{l}C_{l} / C_{d}[-] \\
\quad \operatorname{Max}\end{array}$ & $C_{d}[-]$ Max & $C_{m}[-]$ Max & $\begin{array}{l}C_{l} / C_{d}[-] \\
\quad \operatorname{Max}\end{array}$ & $C_{d}[-]$ Max & $C_{m}[-]$ Max & $\begin{array}{c}C_{l} / C_{d}[-] \\
\text { Ave. }\end{array}$ & $C_{d}[-]$ Ave. & $C_{m}[-]$ Ave. \\
\hline $\mathrm{G}^{3} \mathrm{U} 1$ & -7.27 & -17.34 & -22.34 & 18.67 & -16.61 & -19.10 & -0.40 & -11.64 & -25.20 \\
\hline $\mathrm{G}^{3} \mathrm{U} 2$ & -7.40 & -14.61 & -18.40 & 18.60 & -17.01 & -20.40 & -0.52 & -11.87 & -26.14 \\
\hline $\mathrm{G}^{3} \mathrm{U} 3$ & -4.77 & 0.378 & -14.68 & 22.26 & -52.74 & -70.43 & -0.9 & -18.00 & -69.32 \\
\hline $\mathrm{G}^{3} \mathrm{U} 4$ & -2.77 & 0.45 & -15.60 & 223.26 & -53.30 & -71.34 & 3.03 & -19.02 & -70.30 \\
\hline $\mathrm{G}^{3} \mathrm{U} 5$ & 9.89 & -12.65 & -15.48 & 19.83 & -15.55 & -16.50 & 14.33 & -10.70 & -18.16 \\
\hline $\mathrm{G}^{3} \mathrm{U} 6$ & -3.88 & -9.60 & -9.11 & 11.56 & -27.02 & -41.31 & 3.32 & -13.17 & -32.51 \\
\hline $\mathrm{G}^{3} \mathrm{U} 7$ & 8.77 & -19.60 & -23.10 & 21.10 & -30.41 & -40.56 & 12.84 & -15.78 & -30.78 \\
\hline \multicolumn{10}{|c|}{ Lower Surface Modifications } \\
\hline \multicolumn{10}{|c|}{$2^{\text {nd }}$ Generation: Trailing-vortex Modifications } \\
\hline & \multicolumn{3}{|c|}{ Transonic Phase \% $\Delta$} & \multicolumn{3}{|c|}{ Dynamic Stall Phase \% $\Delta$} & \multicolumn{3}{|c|}{ Average of One Rotor Cycle \% $\Delta$} \\
\hline Model & $\begin{array}{c}C_{l} / C_{d}[-] \\
\operatorname{Max}\end{array}$ & $C_{d}[-] \mathrm{Max}$ & $C_{m}[-]$ Max & $\begin{array}{c}C_{l} / C_{d}[-] \\
\quad \operatorname{Max}\end{array}$ & $C_{d}[-] \mathrm{Max}$ & $C_{m}[-]$ Max & $\begin{array}{c}C_{l} / C_{d}[-] \\
\text { Ave. }\end{array}$ & $C_{d}[-]$ Ave. & $C_{m}$ [-] Ave. \\
\hline $\mathrm{G}^{2} \mathrm{~L} 1$ & 25.20 & -16.40 & -15.59 & 34.60 & -16.10 & -19.82 & 16.74 & -9.031 & -3.43 \\
\hline $\mathrm{G}^{2} \mathrm{~L} 2$ & 44.88 & -7.59 & 3.20 & 9.31 & -25.20 & -31.64 & 22.58 & -10.15 & 9.86 \\
\hline $\mathrm{G}^{2} \mathrm{~L} 3$ & 27.78 & -7.82 & 0.06 & 11.62 & -19.69 & -24.02 & 19.20 & -10.42 & -5.39 \\
\hline \multicolumn{10}{|c|}{$3^{\text {rd }}$ Generation: Body-trapped vortex Modifications } \\
\hline & \multicolumn{3}{|c|}{ Transonic Phase \% $\Delta$} & \multicolumn{3}{|c|}{ Dynamic Stall Phase \% $\Delta$} & \multicolumn{3}{|c|}{ Average of One Rotor Cycle \% $\triangle$} \\
\hline Model & $\begin{array}{c}C_{l} / C_{d}[-] \\
\quad \operatorname{Max}\end{array}$ & $C_{d}[-] \mathrm{Max}$ & $C_{m}[-]$ Max & $\begin{array}{c}C_{l} / C_{d}[-] \\
\quad \operatorname{Max}\end{array}$ & $C_{d}[-]$ Max & $C_{m}[-]$ Max & $\begin{array}{c}C_{l} / C_{d}[-] \\
\text { Ave. }\end{array}$ & $C_{d}[-]$ Ave. & $C_{m}$ [-] Ave. \\
\hline $\mathrm{G}^{3} \mathrm{~L} 1$ & 1.16 & -13.55 & -14.96 & 22.03 & -21.25 & -24.66 & 6.22 & -9.47 & -17.78 \\
\hline $\mathrm{G}^{3} \mathrm{~L} 2$ & 1.52 & -8.00 & -4.78 & 15.01 & -17.63 & -19.74 & 7.10 & -8.49 & -15.61 \\
\hline $\mathrm{G}^{3} \mathrm{~L} 3$ & 67.38 & -5.19 & 11.60 & 11.10 & -21.58 & -22.83 & 27.83 & -7.14 & 37.54 \\
\hline $\mathrm{G}^{3} \mathrm{~L} 4$ & 70.81 & -2.11 & 20.46 & 7.50 & -30.87 & -30.80 & 28.37 & -7.83 & 32.53 \\
\hline $\mathrm{G}^{3} \mathrm{~L} 5$ & 13.29 & -11.73 & -9.80 & 21.73 & -14.72 & -14.10 & 15.70 & -10.72 & -18.33 \\
\hline $\mathrm{G}^{3} \mathrm{~L} 6$ & 16.10 & -10.40 & -7.48 & 15.23 & -20.75 & -25.67 & 11.16 & -9.98 & -11.83 \\
\hline $\mathrm{G}^{3} \mathrm{~L} 7$ & 12.83 & -12.69 & -11.10 & 19.10 & -19.40 & -22.33 & 13.58 & -10.68 & -17.65 \\
\hline
\end{tabular}




\subsubsection{SC1095 with $G^{3} U 2$ Modification}

The $G^{3} U 2$ flow control modification was developed after an extensive partial optimization, which objective was to decrease shock induced drag during the transonic phase, as well as to control vortex shedding during the dynamic stall phase via the interaction between the vortex trapped inside the SGTV cavity and the dynamic stall vortex over the airfoil upper surface.

The airfoil has the modification installed at $50 \% c$ with a depth of $18 \% t$ and length of $16.4 \% c$ towards the trailing edge. Fig. 4.39 shows the outline of the SC1095 airfoil with the $\mathrm{G}^{3} \mathrm{U} 2$ modification, while Figs. 4.40 displays the aerodynamic coefficients. Finally, on Fig. 4.41, the pressure coefficient as well as the Mach number contours, superimposed by vorticity are shown.

First, let observe the peculiarities of the flow due to the fluctuating nature of the freestream. Note from the flow visualization plots on Fig. 4.41, that unlike for constant freestream dynamic stall, where flow separation occurs only beyond the static stall angle of attack (typically beyond $12^{\circ}$ ), for the fluctuating freestream case the flow separation is present for all of the upstroke phase $\left(0^{\circ}-20^{\circ}\right)$. At low $A o A s$, flow separation occurs due to the shock wave - boundary layer interaction at transonic speeds (Frames A-E on Fig. 41, corresponding to $\operatorname{AoA} 0^{\circ}-12^{\circ}$ ). The vorticity plots illustrate clearly the regions of boundary layer separation. As a result of this, the airfoil effectively enters the "dynamic stall" dominated phase already with a massive flow separation of the upper surface (Frame F on Fig. 4.41). Then, a dynamic stall vortex appears to be formed on Frame G, followed by the classical trailing edge vortex in Frame H. 
The effect of the STVG appears to be that:

a) During the transonic phase, it changes the pressure distribution on the upper surface during the transonic phase in a favorable way (see Frames C-E in Fig. 4.41). Note that a larger portion of the airfoil surface is exposed to low pressure. This causes lift increase (see Fig. 4.40, points C-E). This effect is in line with the observations made for the same STVG geometry for constant freestream transonic flow in Section 4.3. At the same time, this leads to lower drag likely due to the lower height of the separated region (Frames C-D in Fig. 4.41). And, since the pressure is redistributed in a way that its resultant force acts more forward, pitching moment decreases as well (see Frames C-D again). All these affects can also be seen as the lift curve being shifted by about $1.5^{\circ}$ phase delay relative to the clean airfoil.

b) During the dynamic stall phase, the $1.5^{\circ}$ phase delay is maintained throughout the rest of the cycle for lift (Frames G-H), while a (favorable) decrease in drag and pitching moment can be observed.

An important effect of the modification was to generate a weaker shockwave during the transonic phase, as established earlier in Section 4.3 (see the pressure plots of Frames $\mathrm{A}, \mathrm{B}$ and $\mathrm{K}$ in Fig. 4.41), and to shift the peaks in the hysteresis loops by approximately $1.5^{\circ}$ later when compared to the original airfoil. This causes the first and second stall events of the modified airfoil to happen with a delay in comparison to the baseline airfoil. For example, the first stall event, which is due to shock-induced boundary layer separation, combined with the increase in the angle of attack happened at angle of attack of $10.5^{\circ}$ for the modified airfoil while it was $9^{\circ}$ for the baseline airfoil (Fig. 4.40). 

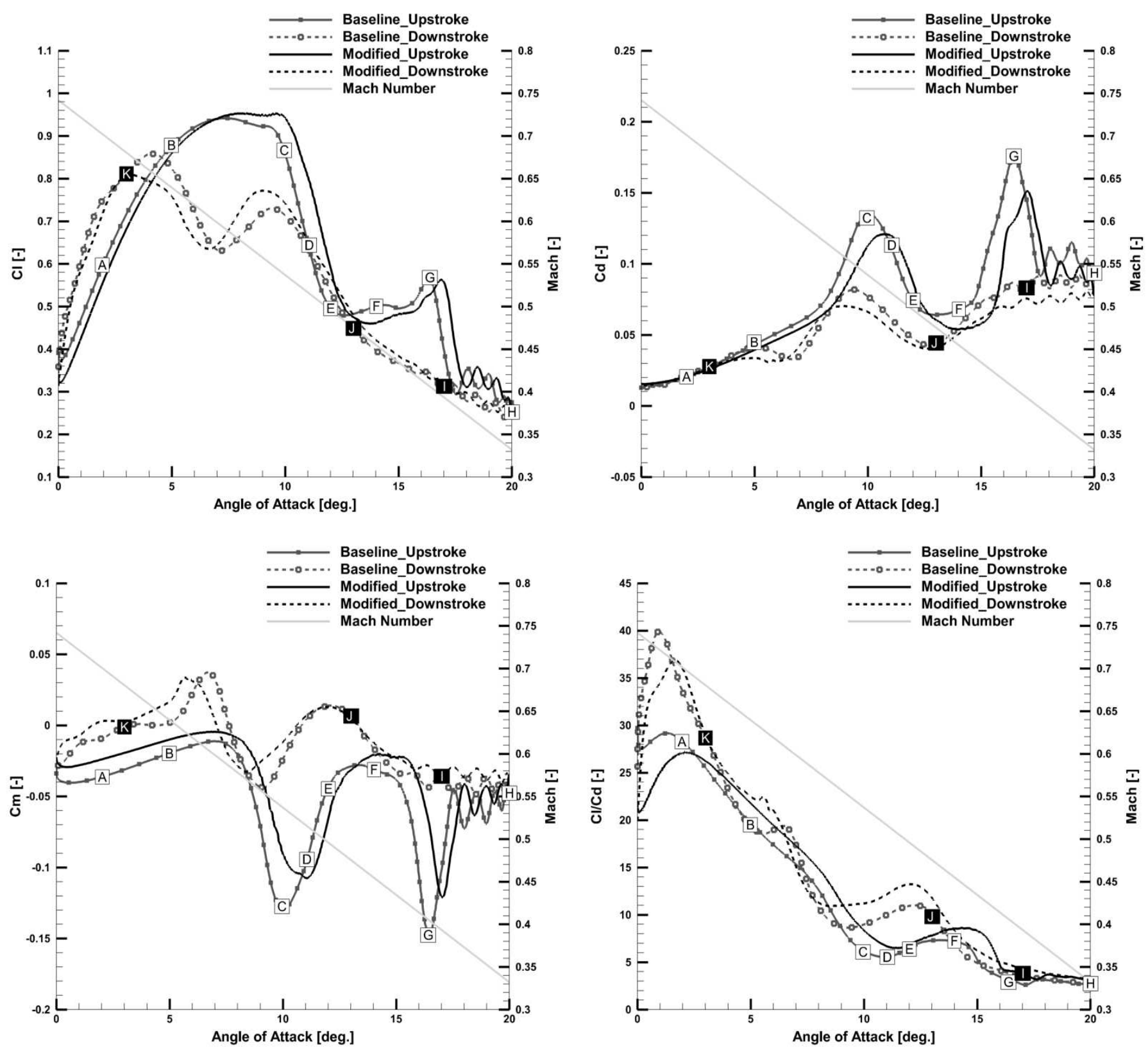

Figure 4.40 Aerodynamic loads SC1095 airfoil with $\mathrm{G}^{3} \mathrm{U} 2$ modification. $M=$

$0.537 \pm 0.205, f=4.25 \mathrm{~Hz}, \operatorname{Re}=6.1 \times 10^{6}$ and $\alpha=10^{\circ} \pm 10^{\circ}$. 
The last portion of the upstroke phase (Frames G-H), along with the beginning of the downstroke phase (Frames I-K) is dominated for both airfoils by shedding of multiple vortices, which is associated with the oscillations in the aerodynamic loads in Fig. 4.40.

The average loads of the modified airfoil over a rotor cycle was given in Table 4.4 Note that - over one rotor revolution - the lift-to-drag ratio remains virtually unchanged (a reduction of $-0.5 \%$ only) while drag is reduced by $12 \%$ and pitching moment by as much as $26 \%$ a very promising result in overall. 

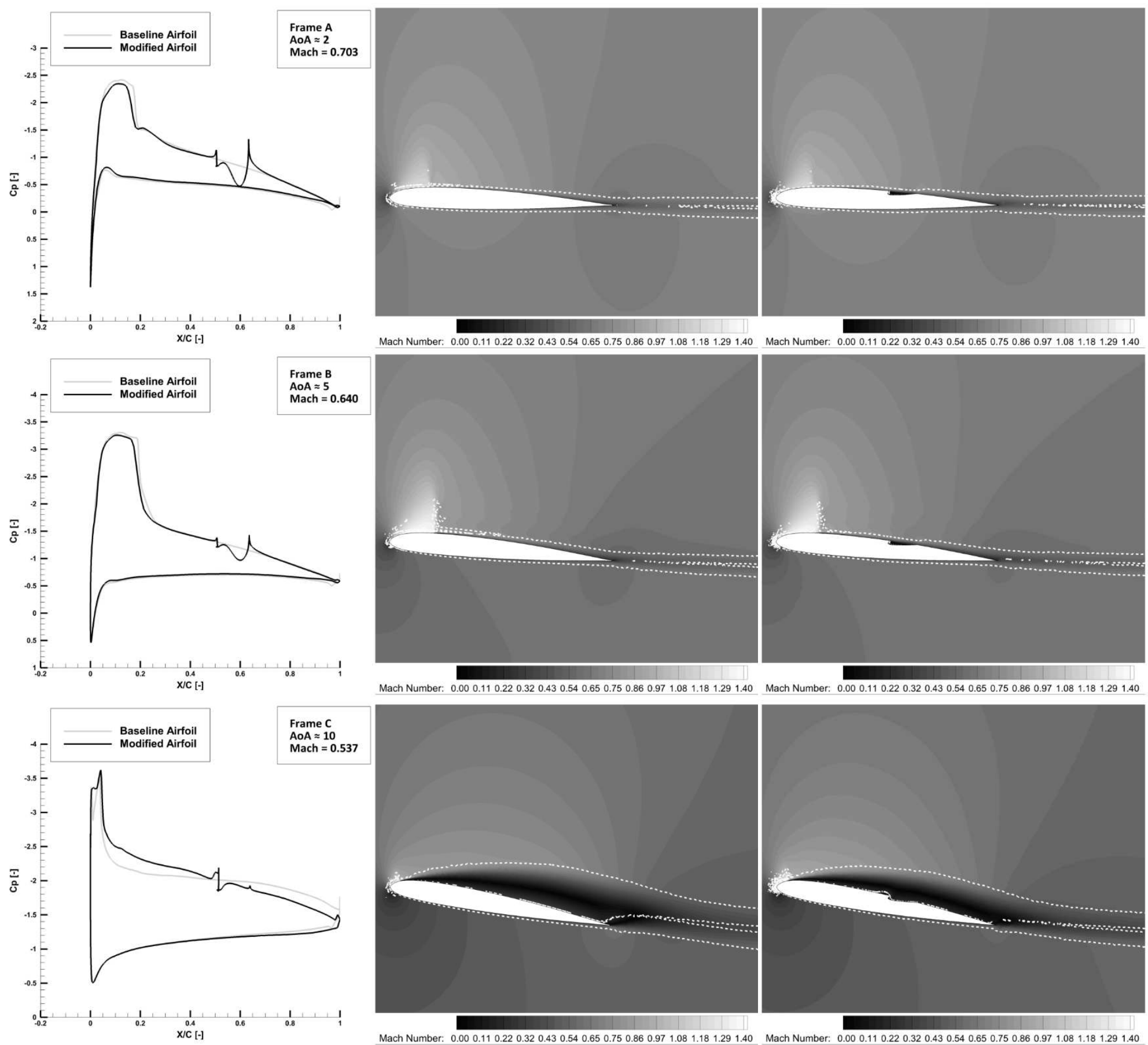

Mach Number: $0.00 \quad 0.110 .220 .320 .430 .540 .650 .750 .860 .971 .081 .181 .291 .40$ Mach Number: $0.000 .110 .220 .320 .43 \quad 0.540 .650 .750 .860 .971 .081 .181 .291 .40$
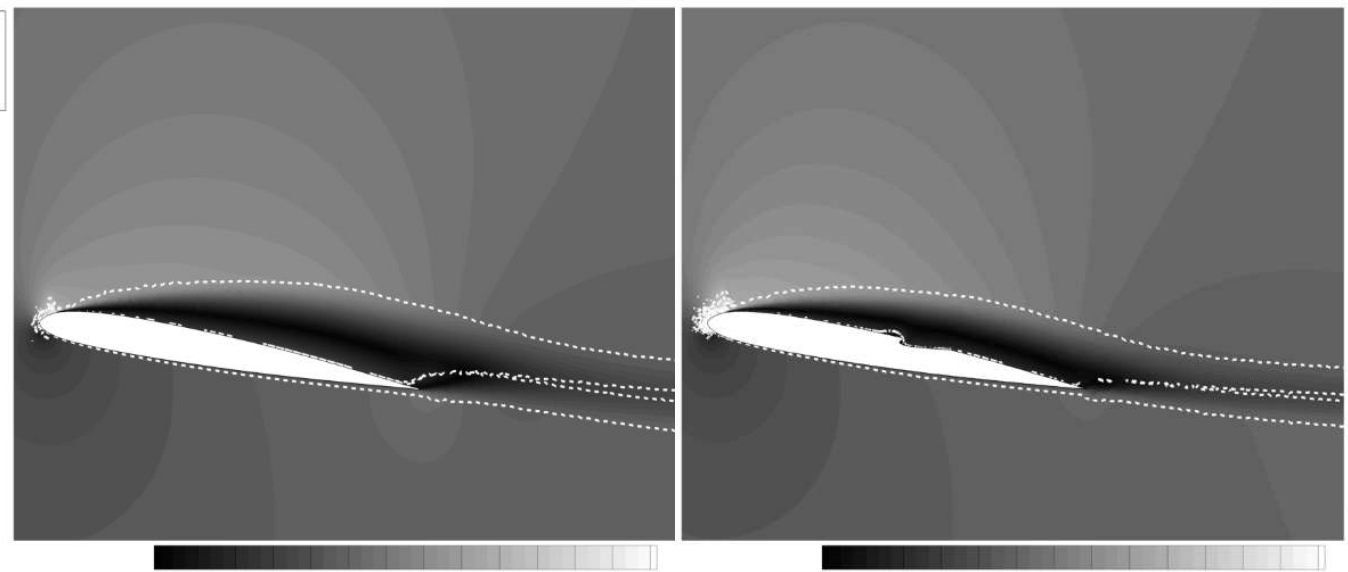

Mach Number: $0.000 .110 .220 .320 .430 .54 \quad 0.650 .750 .860 .97$ 1.08 1.18 1.29 1.40 Mach Number: 0.000 .110 .220 .320 .430 .540 .650 .750 .860 .971 .081 .181 .291 .40

Figure 4.41 Pressure coefficient, Mach number contours superimposed with maximum instantaneous vorticity lines (white dashed boundaries) for the SC1095 airfoil and SC1095 with $\mathrm{G}^{3} \mathrm{U} 2 . M=0.537 \pm 0.205, f=4.25 \mathrm{~Hz}, \operatorname{Re}=6.1 \times 10^{6}$ and $\alpha=10^{\circ} \pm 10^{\circ}$. 

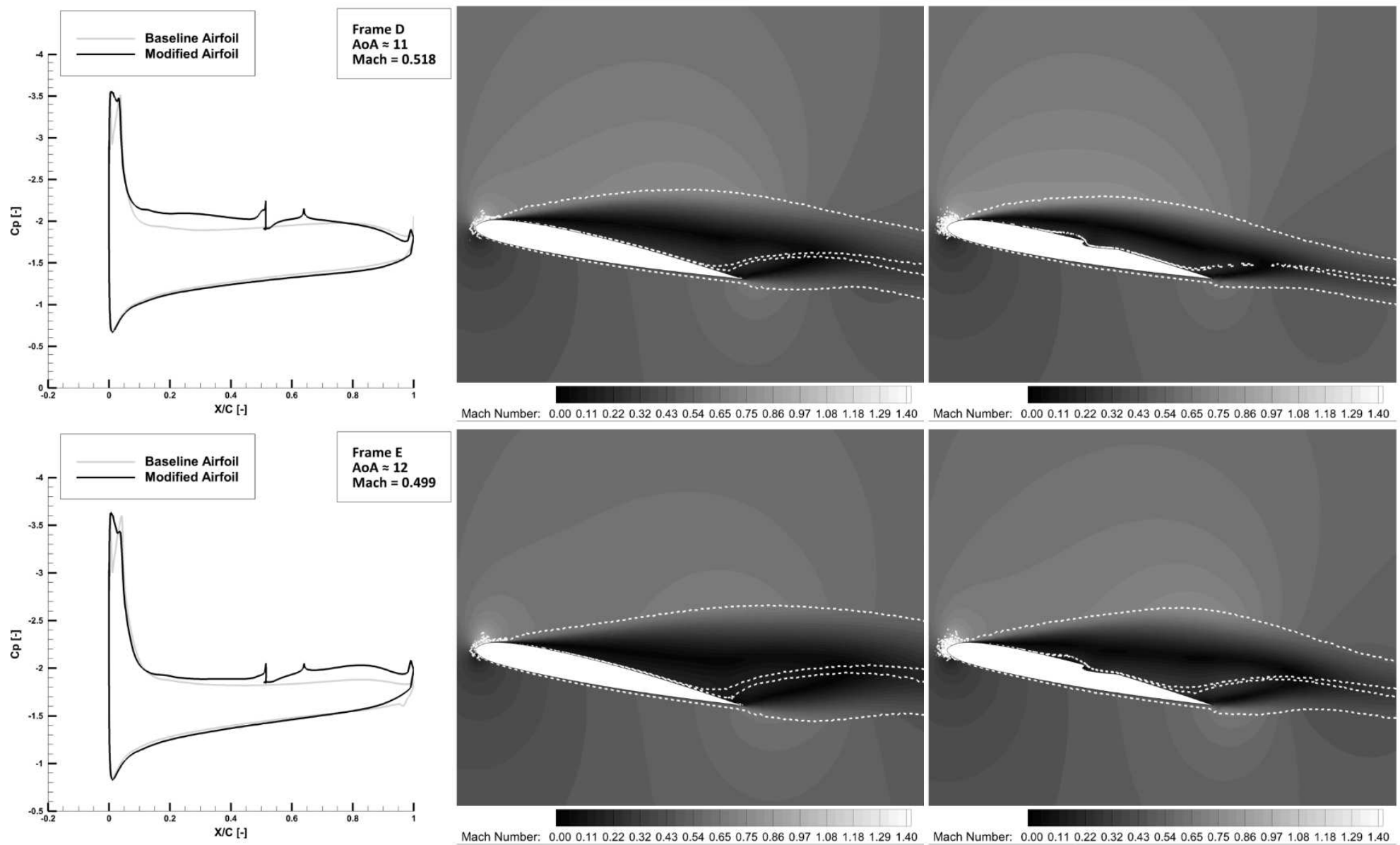

Mach Number: $0.000 .110 .22 \quad 0.320 .430 .54 \quad 0.650 .750 .860 .971 .081 .181 .291 .40 \quad$ Mach Number: 0.000 .110 .220 .320 .430 .540 .650 .750 .860 .971 .081 .181 .291 .40
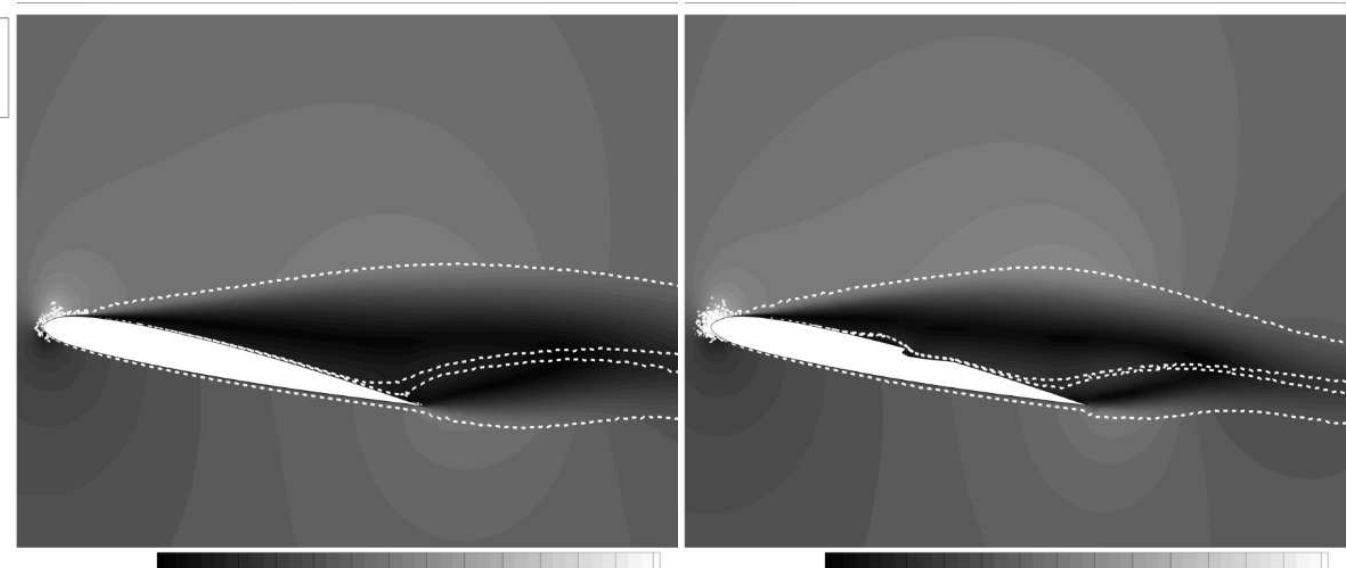

Mach Number: $0.000 .110 .220 .320 .430 .540 .650 .750 .860 .971 .081 .181 .291 .40 \quad$ Mach Number: 0.000 .110 .220 .320 .430 .540 .650 .750 .860 .971 .081 .181 .291 .40
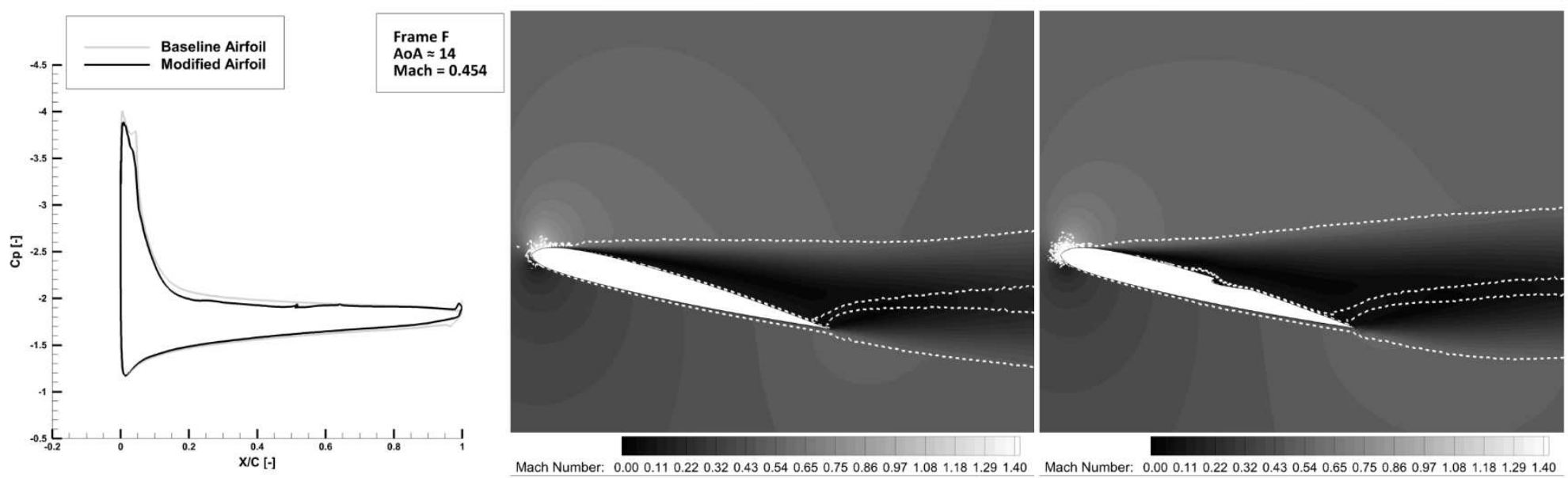

Figure 4.41 (Continued) Pressure coefficient, Mach number contours superimposed with maximum instantaneous vorticity lines (white dashed boundaries) for the SC1095 airfoil and $\mathrm{SC} 1095$ with $\mathrm{G}^{3} \mathrm{U} 2 . M=0.537 \pm 0.205, f=4.25 \mathrm{~Hz}, \operatorname{Re}=6.1 \times 10^{6}$ and $\alpha=$

$$
10^{\circ} \pm 10^{\circ}
$$



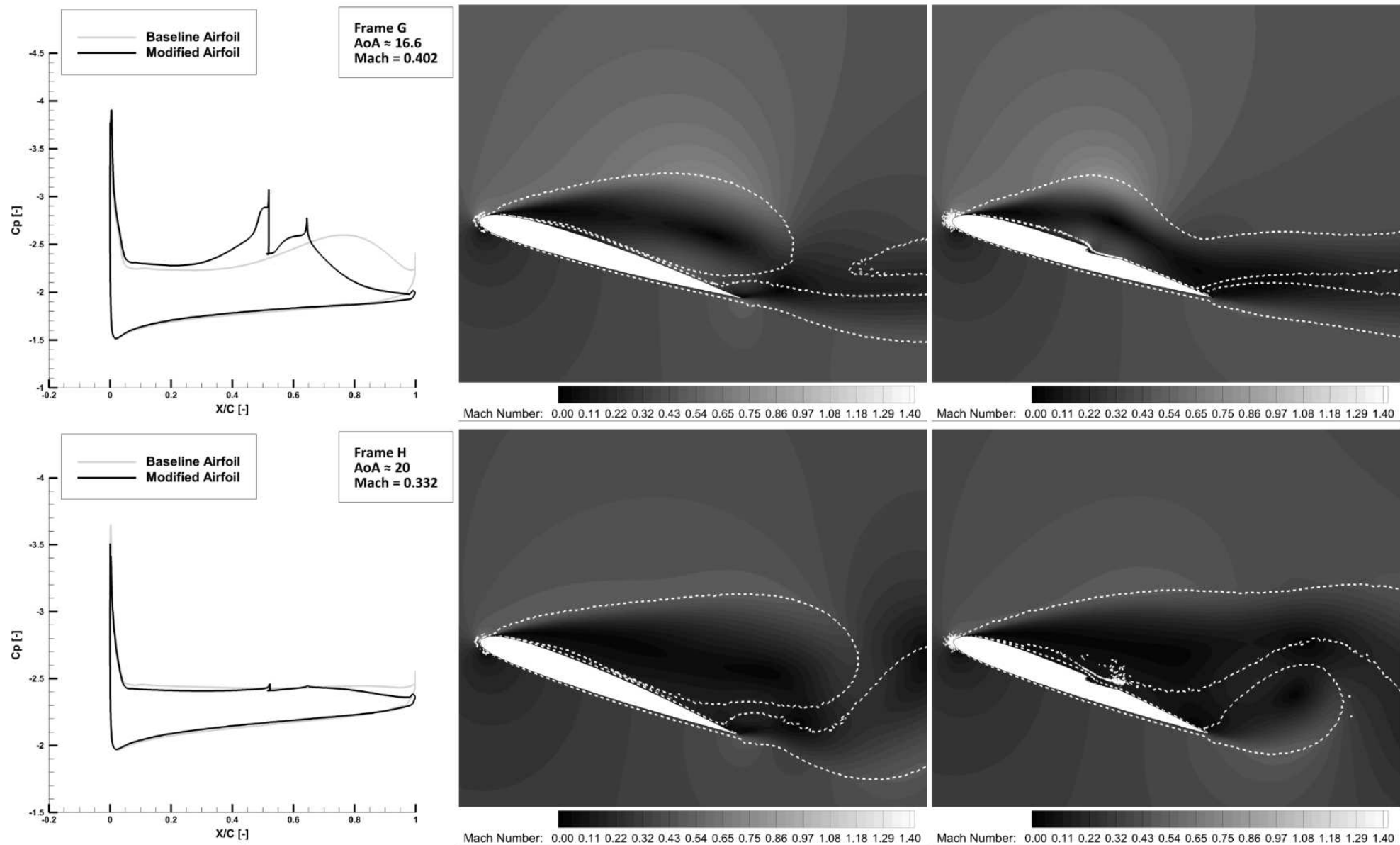

Mach Number: 0.000 .110 .220 .320 .430 .540 .650 .750 .860 .971 .081 .181 .291 .40 Mach Number: 0.000 .110 .220 .320 .430 .540 .650 .750 .860 .971 .081 .181 .291 .40
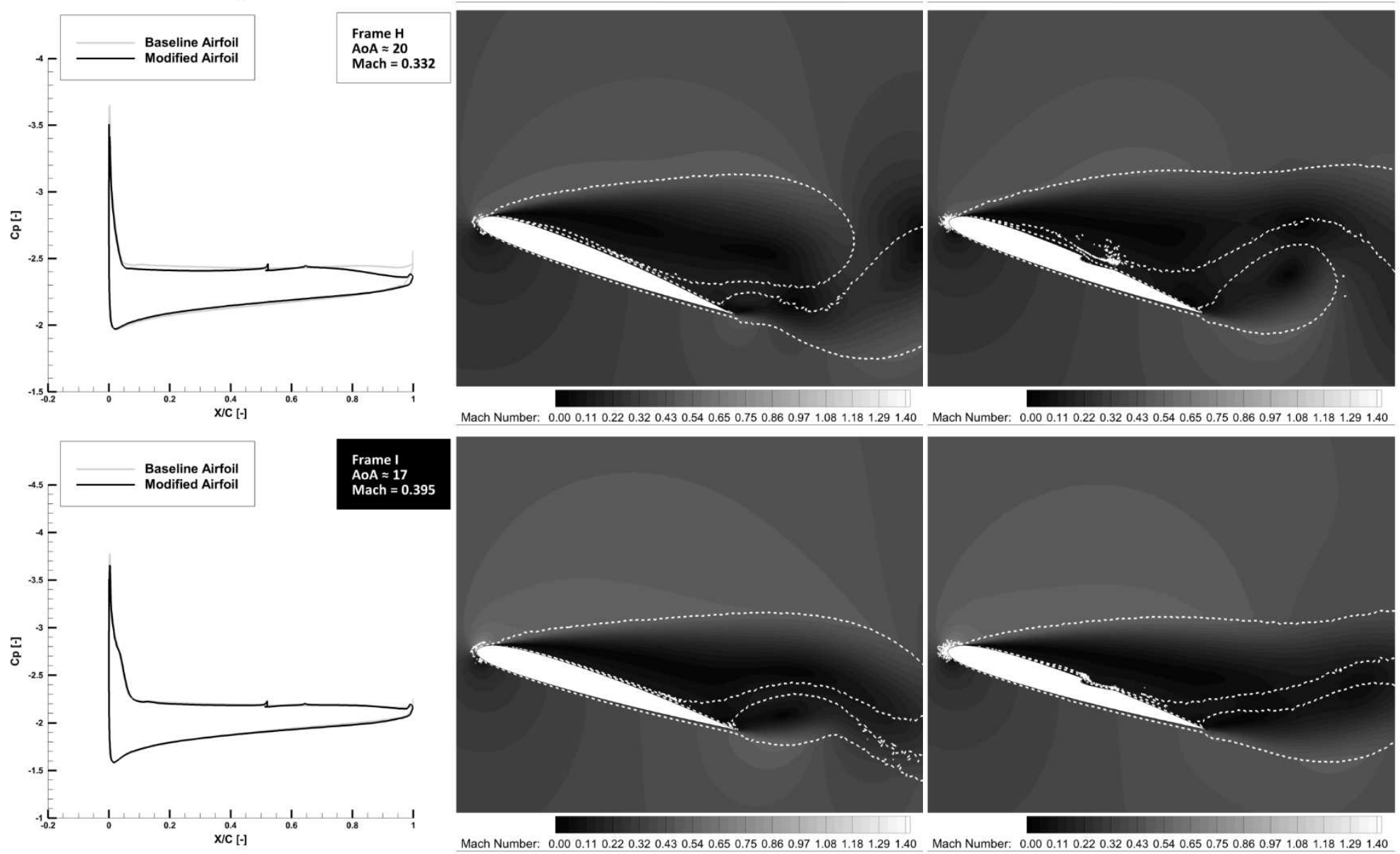

Figure 4.41 (Continued) Pressure coefficient, Mach number contours superimposed

with maximum instantaneous vorticity lines (white dashed boundaries) for the SC1095 airfoil and $\mathrm{SC} 1095$ with $\mathrm{G}^{3} \mathrm{U} 2 . M=0.537 \pm 0.205, f=4.25 \mathrm{~Hz}, \operatorname{Re}=6.1 \times 10^{6}$ and $\alpha=$

$$
10^{\circ} \pm 10^{\circ}
$$



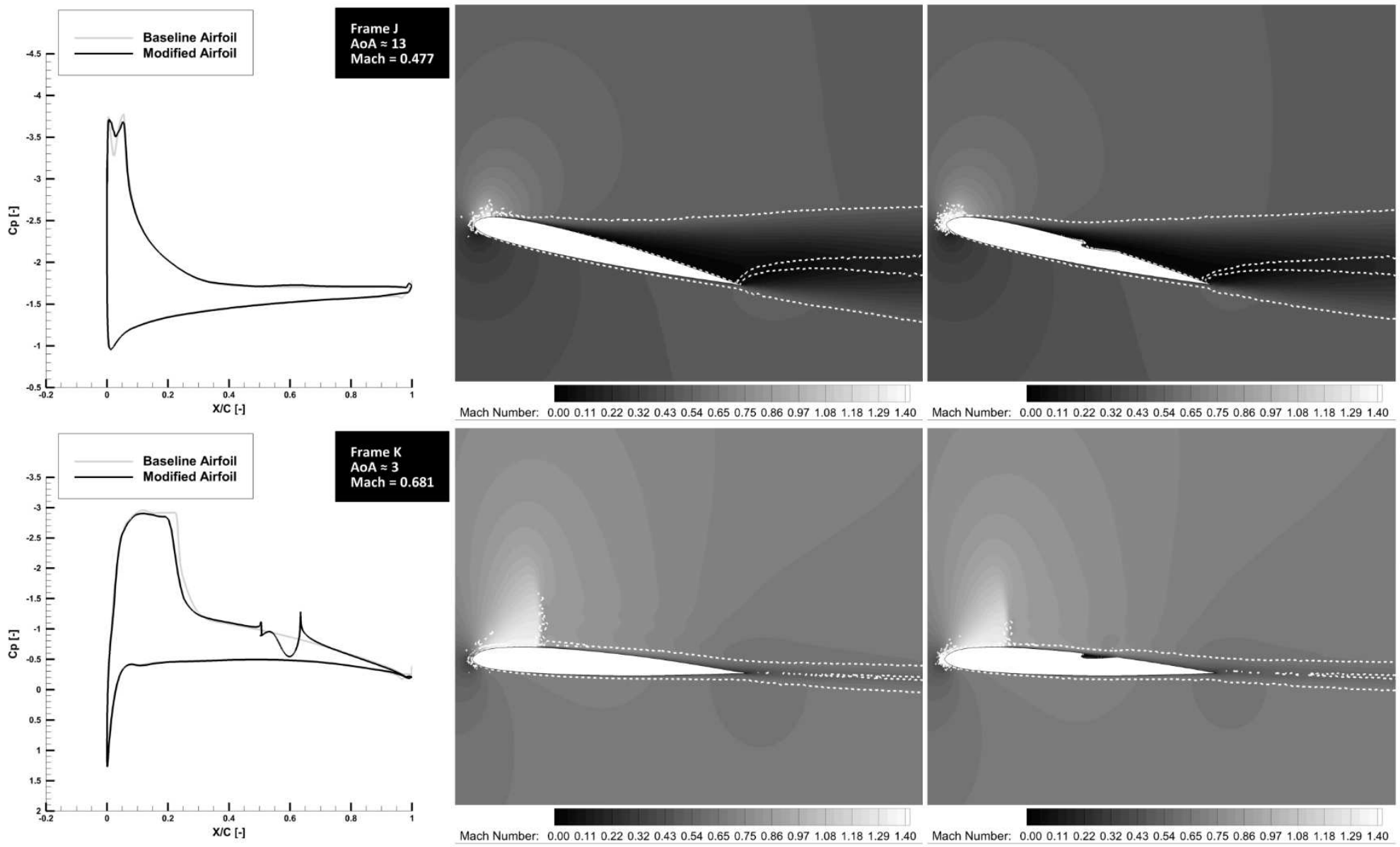

Figure 4.41 (Continued) Pressure coefficient, Mach number contours superimposed with maximum instantaneous vorticity lines (white dashed boundaries) for the SC1095 airfoil and $\mathrm{SC} 1095$ with $\mathrm{G}^{3} \mathrm{U} 2 . M=0.537 \pm 0.205, f=4.25 \mathrm{~Hz}, \operatorname{Re}=6.1 \times 10^{6}$ and $\alpha=$

$$
10^{\circ} \pm 10^{\circ}
$$

\subsubsection{SC1095 with $G^{2} L 1$ modification}

As have been shown in Section 4.3, the lower surface modifications have the ability to increase the lift-to-drag ratio for the full range of angles of attack by increasing the pressure on the lower surface of the airfoil, where the modification is implemented (Section 4.3). Several designs have been proposed in the current paper, but only the results for $\mathrm{G}^{2} \mathrm{~L} 1$ type will be discussed. This had the STVG placed near the trailing-edge 
on the lower surface. The STVG geometry is a small step-like shape, starting at $0.9 c$. The location was dictated by structural design considerations: the smaller the modification, the better from structural point of view. The cavity is extended to the trailing-edge, and its depth is $0.25 t$, (relative to the local thickness where the modification starts). Fig. 4.39 illustrates the geometry.

Fig. 4.42 provides the aerodynamic coefficients of the modified airfoil compared to that of the baseline airfoil. From this it can be seen that the $G^{2} L 1$ modification outperforms the baseline airfoil in terms of lift as well as the lift-to-drag ratio for both the transonic and dynamic stall phases. It also decreases the drag and the nose-down pitching moment during both phases.

This time, the STVG appears to cause an opposite overall effect in comparison to the previous case. Instead of delaying events by $1.5^{\circ}$, it causes them to appear earlier, at about $1^{\circ}$. This appears to be certainly true for lift and drag during the upstroke.

Examining the flow mechanism, again, the same major flow features are present as for the previous case. In the transonic phase, however, the increase in lift is likely not due to modifying the upper surface flow, but the lower one. Here, the pressure appears to rise within the cavity, causing larger difference between the upper and lower surface, hence leading to increased lift (see the pressure distribution of Frames A-F in Fig. 4.43). This observation is in accordance with Section 4.3.

In the dynamic stall phase, the same effect dominates: although a dynamic stall vortex and trailing edge vortex are created as usual, they are counter-balanced by a highpressure region on the lower surface, thus increasing lift and decreasing the nose-down pitching moment (see Frames G-H in Fig. 4.43). 
Despite the high oscillation frequency $(4.25 \mathrm{~Hz})$ and the fluctuating freestream, the modified airfoil succeeded in favorably augmenting lift, reducing both drag and pitching moment during the upstroke and downstroke phases, at virtually no penalty as shown previously in Table 4.4.
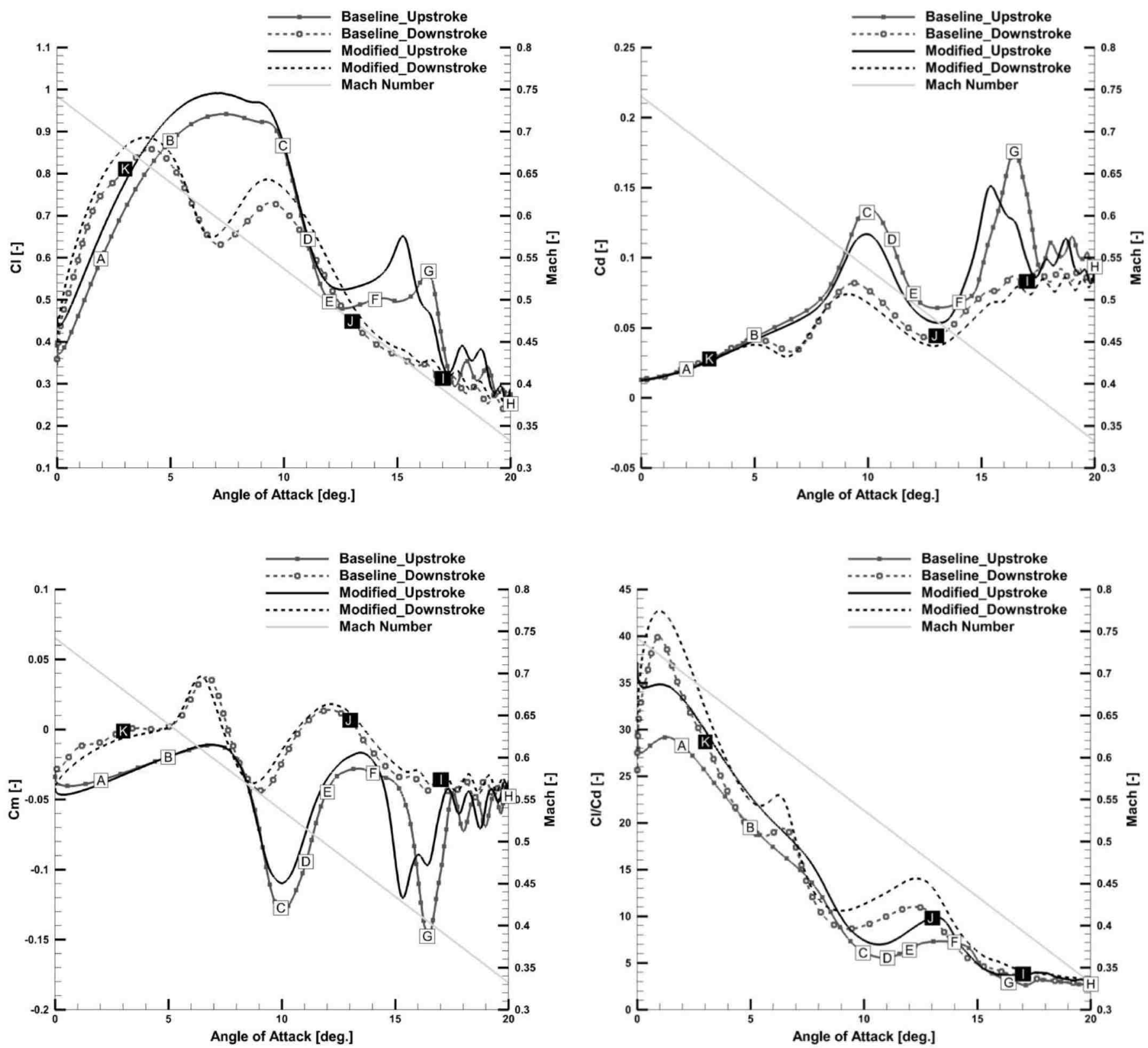

Figure 4.42 Aerodynamic loads SC1095 airfoil with $\mathrm{G}^{2} \mathrm{~L} 1$ modification. $M=$

$$
0.537 \pm 0.205, f=4.25 \mathrm{~Hz}, \operatorname{Re}=6.1 \times 10^{6} \text { and } \alpha=10^{\circ} \pm 10^{\circ} \text {. }
$$



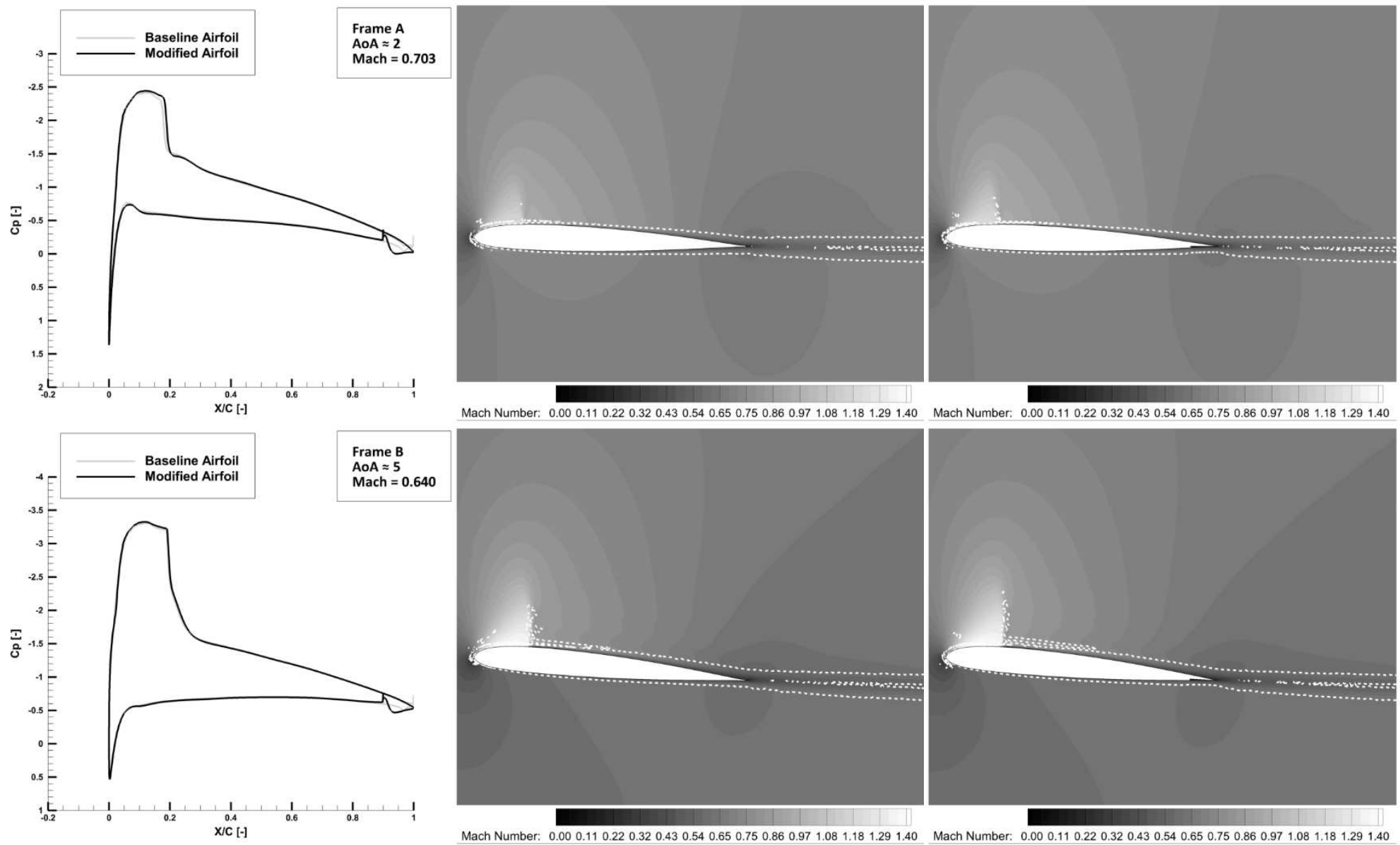

Mach Number: $0.000 .110 .220 .320 .430 .54 \quad 0.650 .750 .860 .971 .081 .181 .291 .40 \quad$ Mach Number: 0.000 .110 .220 .320 .430 .540 .650 .750 .860 .971 .081 .181 .291 .40
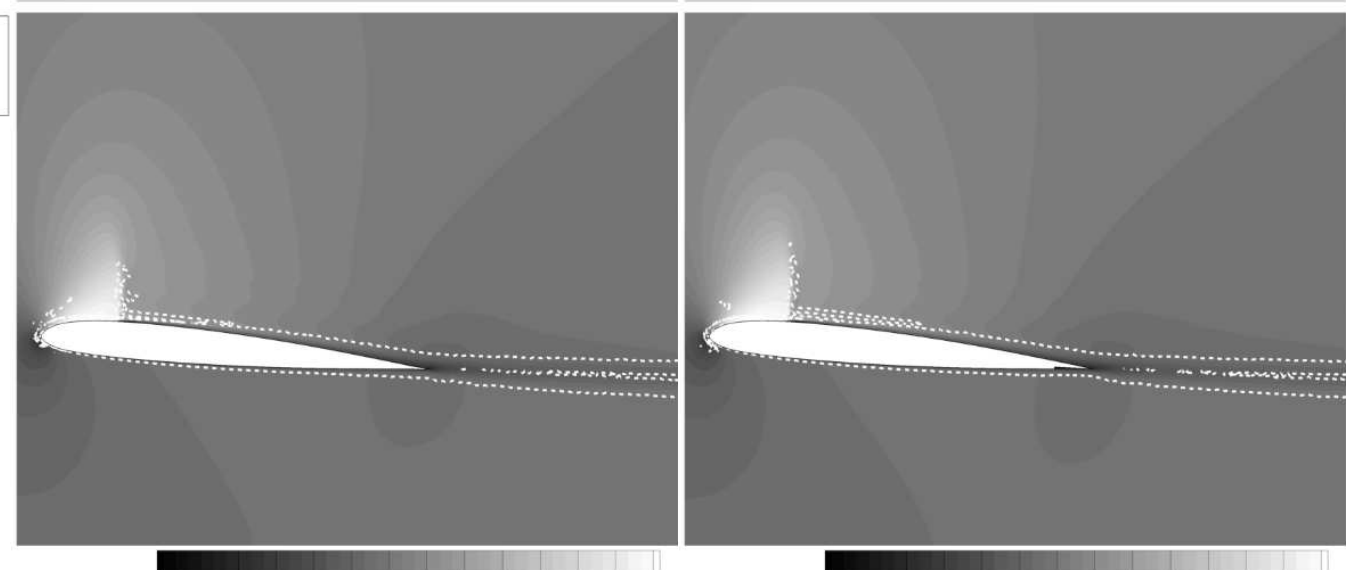

Mach Number: $0.000 .110 .220 .320 .430 .540 .650 .750 .860 .971 .081 .181 .291 .40 \quad$ Mach Number: 0.000 .110 .220 .320 .430 .540 .650 .750 .860 .971 .081 .181 .291 .40
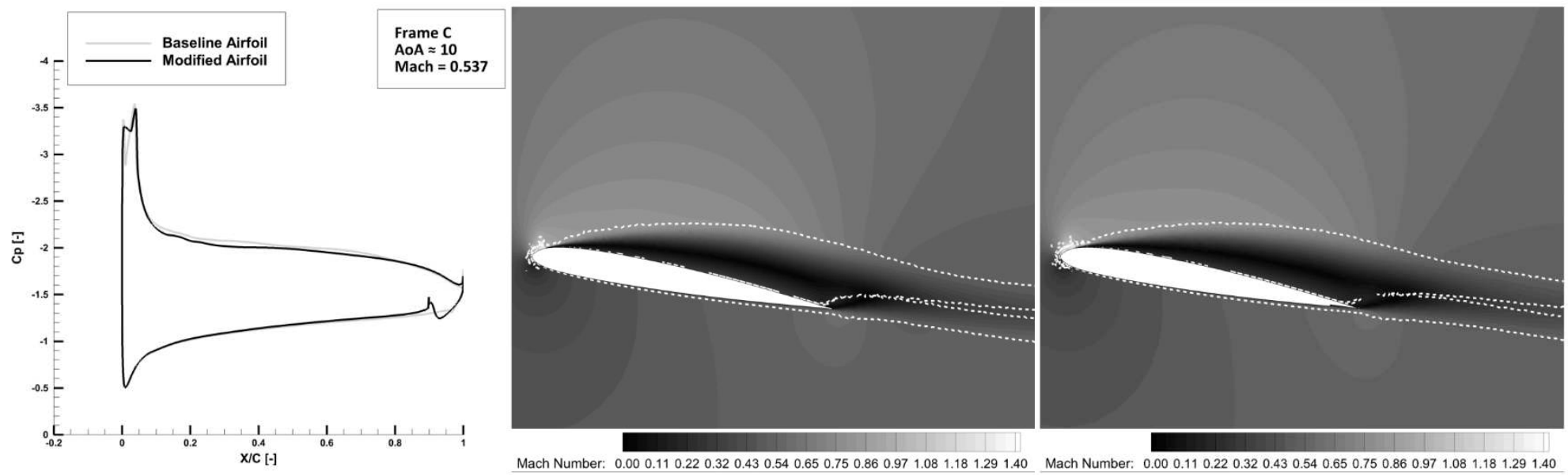

Mach Number: $0.000 .110 .22 \quad 0.320 .430 .540 .650 .750 .860 .971 .081 .181 .291 .40$ Mach Number: 0.000 .110 .220 .320 .430 .540 .650 .750 .860 .971 .081 .181 .291 .40

Figure 4.43 Pressure coefficient, Mach number contours superimposed with maximum instantaneous vorticity lines (white dashed boundaries) for the SC1095 airfoil and SC1095 with $\mathrm{G}^{2} \mathrm{~L} 1 . M=0.537 \pm 0.205, f=4.25 \mathrm{~Hz}, \operatorname{Re}=6.1 \times 10^{6}$ and $\alpha=10^{\circ} \pm 10^{\circ}$. 

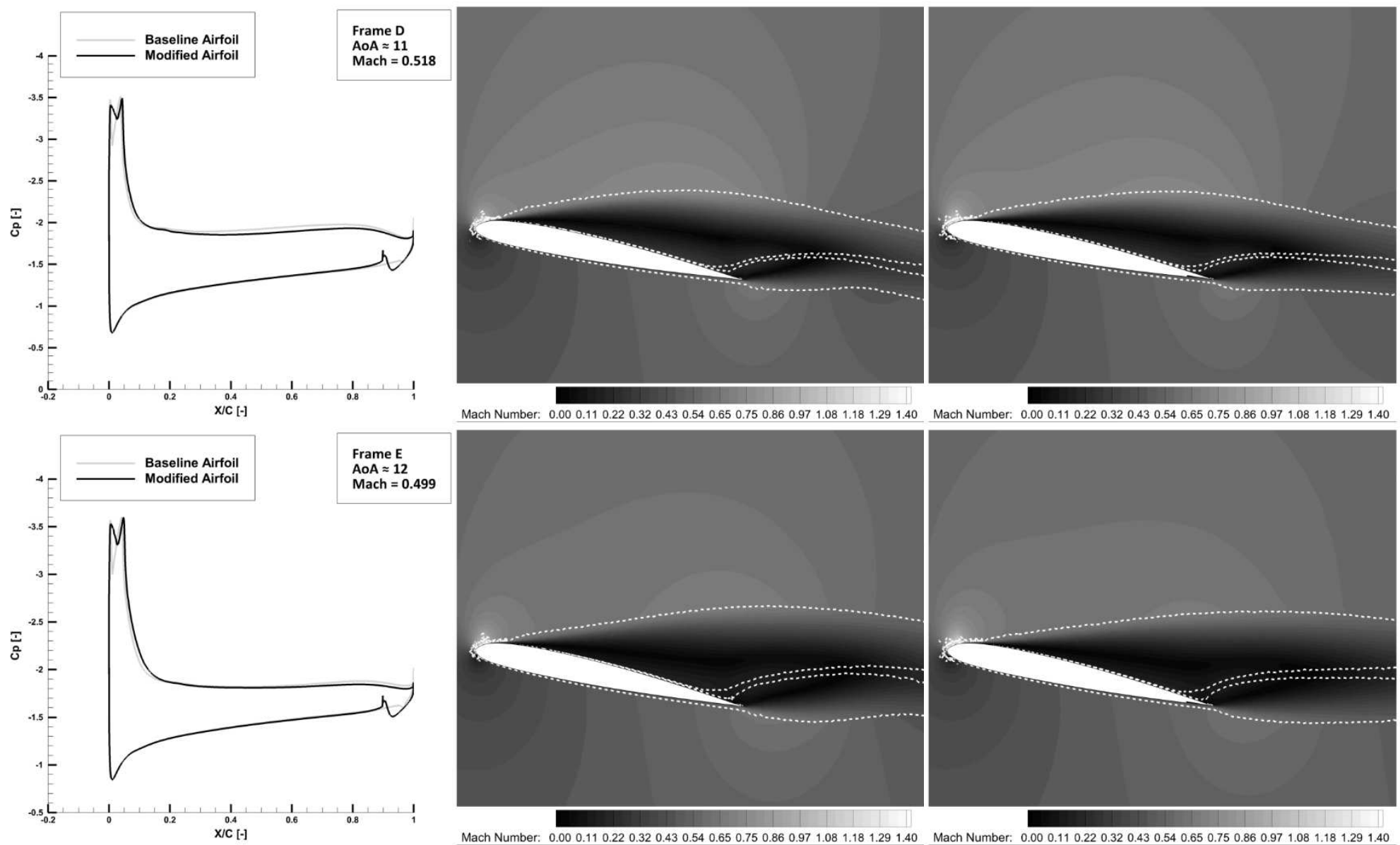

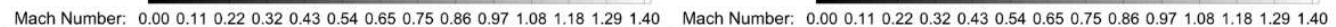

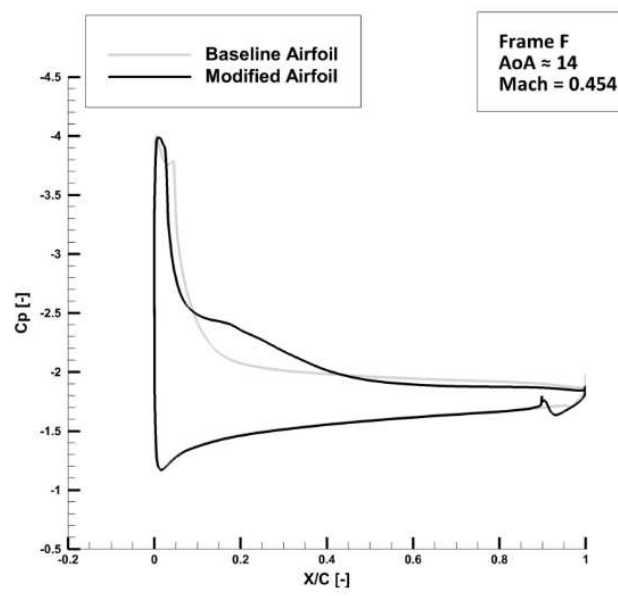

Mach Number: $0.000 .11 \quad 0.22 \quad 0.32 \quad 0.43 \quad 0.540 .650 .750 .860 .971 .081 .181 .29 \quad 1.40$ Mach Number: $0.000 .110 .220 .320 .430 .54 \quad 0.650 .750 .860 .971 .081 .181 .291 .40$
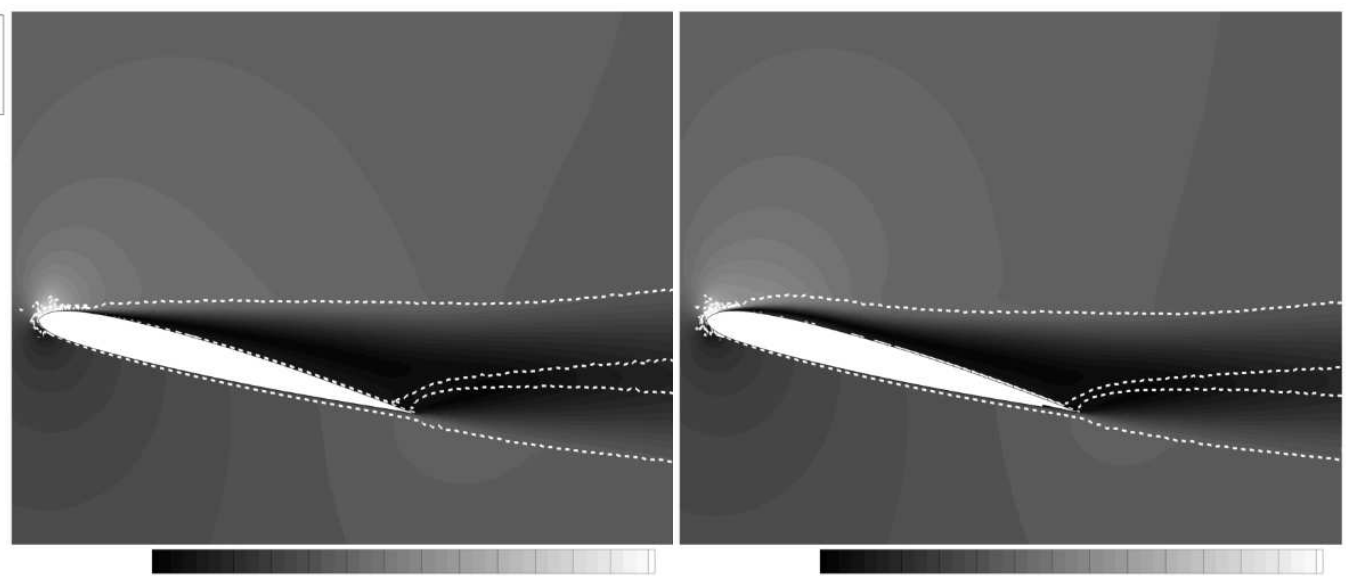

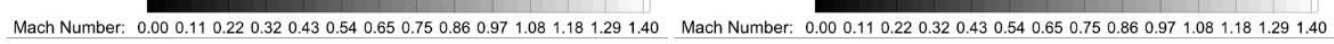

Figure 4.42 (Continued) Pressure coefficient, Mach number contours superimposed

with maximum instantaneous vorticity lines (white dashed boundaries) for the SC1095 airfoil and SC1095 with $\mathrm{G}^{2} \mathrm{~L} 1 . M=0.537 \pm 0.205, f=4.25 \mathrm{~Hz}, \operatorname{Re}=6.1 \times 10^{6}$ and $\alpha=$

$$
10^{\circ} \pm 10^{\circ}
$$



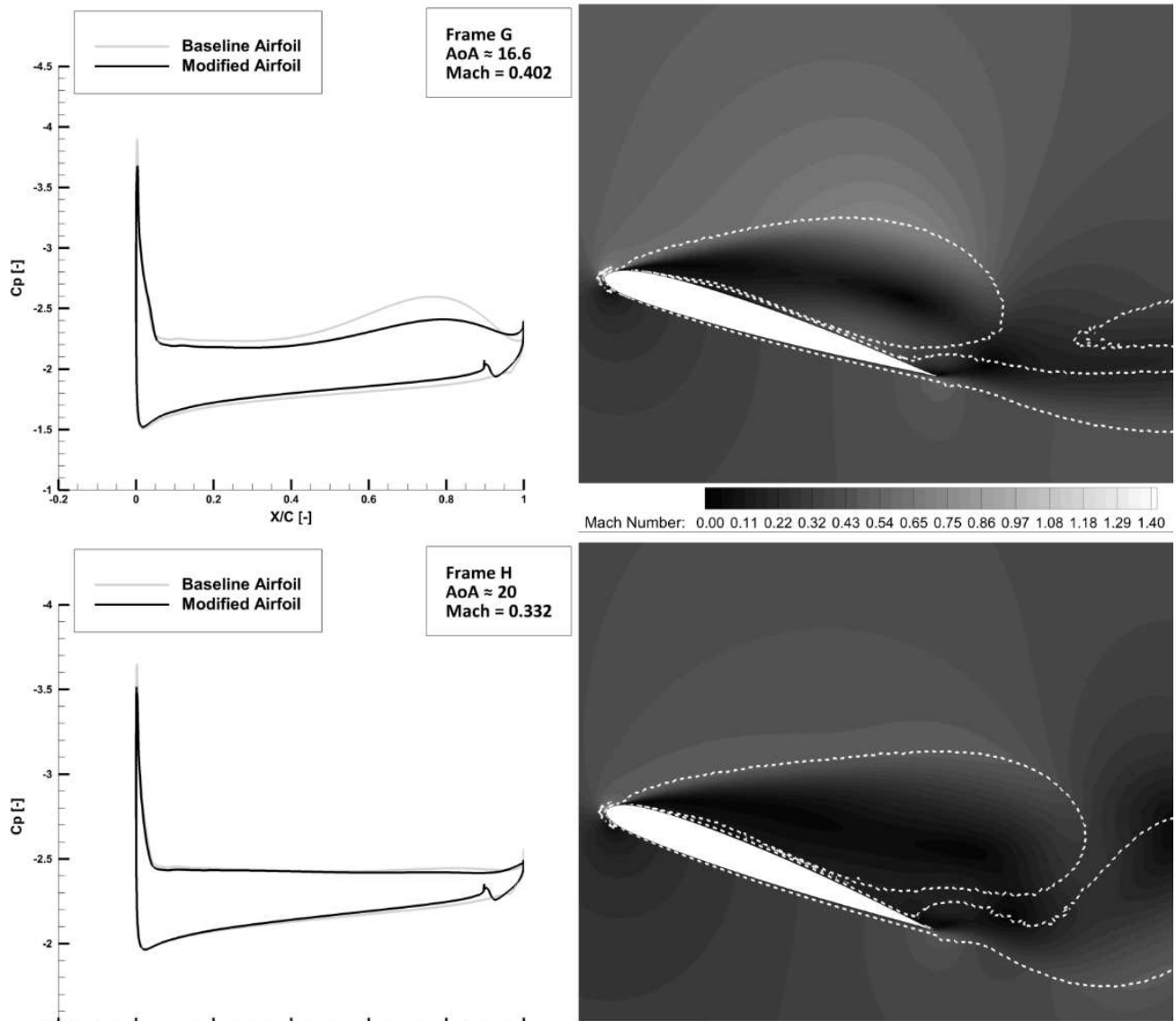

Frame $\mathrm{H}$
$\mathrm{A} O \mathrm{~A} \approx 20$

Mach $=0.33$
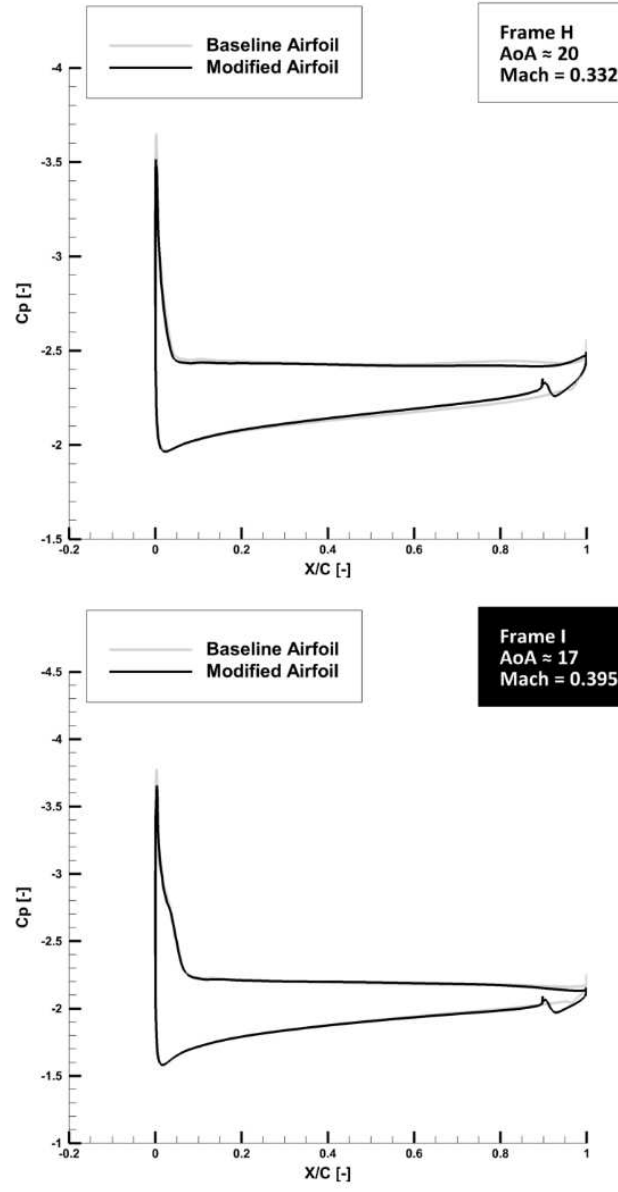

Mach Number: $0.000 .110 .220 .320 .430 .540 .650 .750 .860 .971 .081 .181 .291 .40 \quad$ Mach Number: 0.000 .110 .220 .320 .430 .540 .650 .750 .860 .971 .081 .181 .291 .40
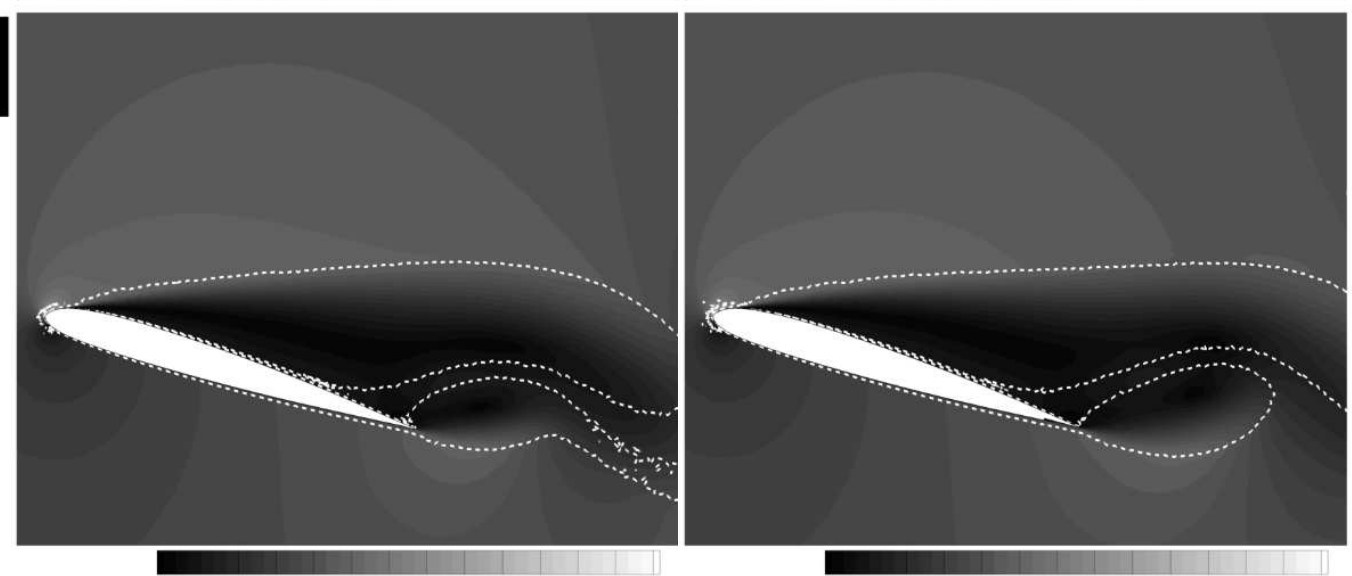

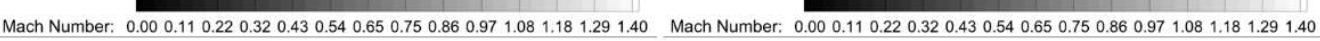

Figure 4.43 (Continued) Pressure coefficient, Mach number contours superimposed

with maximum instantaneous vorticity lines (white dashed boundaries) for the SC1095 airfoil and SC1095 with $\mathrm{G}^{2} \mathrm{~L} 1 . M=0.537 \pm 0.205, f=4.25 \mathrm{~Hz}, \operatorname{Re}=6.1 \times 10^{6}$ and $\alpha=$

$$
10^{\circ} \pm 10^{\circ}
$$



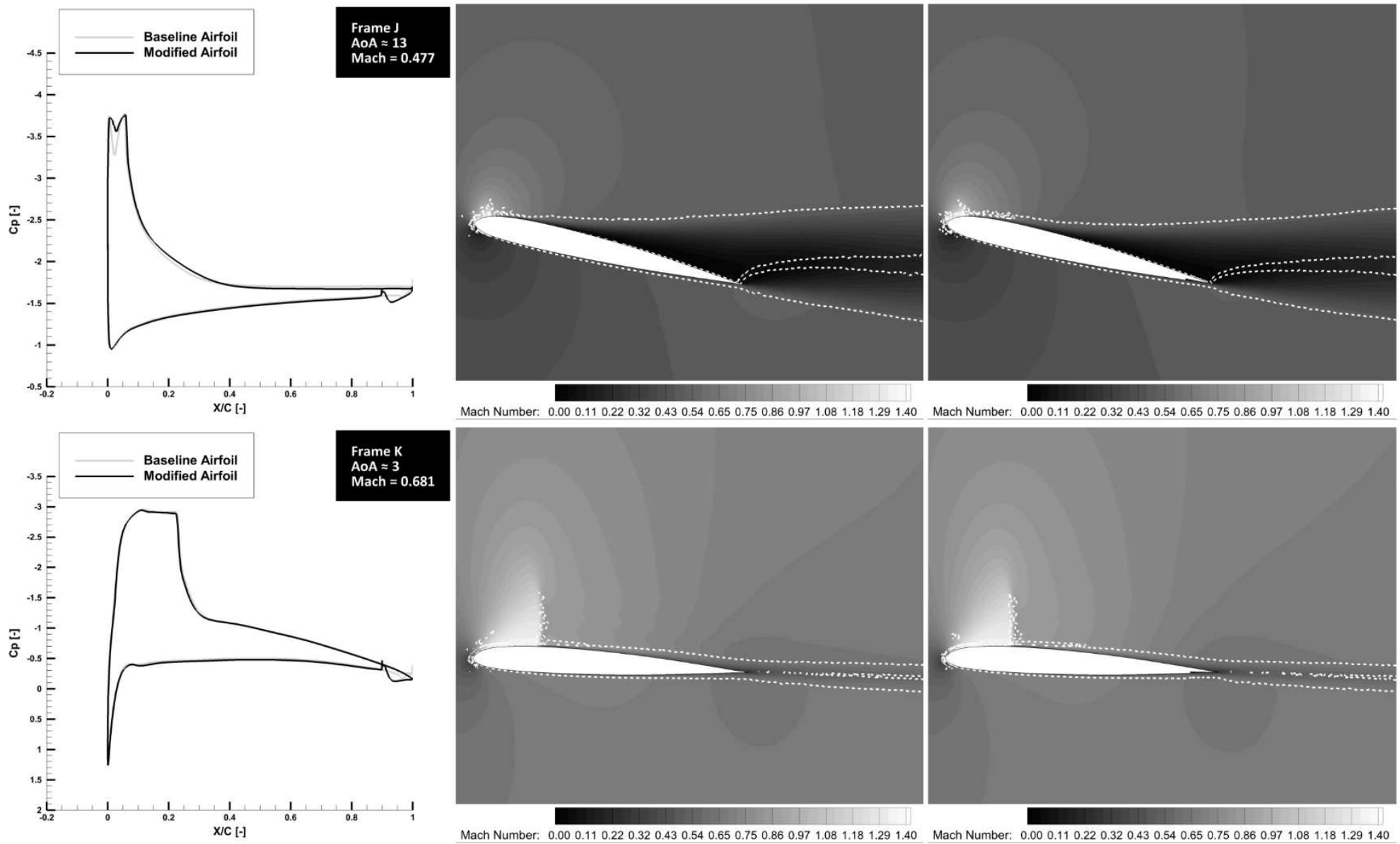

Figure 4.43 (Continued) Pressure coefficient, Mach number contours superimposed with maximum instantaneous vorticity lines (white dashed boundaries) for the SC1095 airfoil and SC1095 with $\mathrm{G}^{2} \mathrm{~L} 1 . M=0.537 \pm 0.205, f=4.25 \mathrm{~Hz}, \operatorname{Re}=6.1 \times 10^{6}$ and $\alpha=$

$$
10^{\circ} \pm 10^{\circ}
$$




\section{Chapter 5}

\section{Conclusions, Contributions and Future Work}

\subsection{Conclusions}

A novel and easy to implement passive flow control concept was investigated via CFD for mitigating the negative effects of transonic flow on the wing section of a commercial jet during cruise, as well as of dynamic stall on the blades of helicopters main rotors in forward flight.

The idea was to create a cavity in one of the airfoil surfaces, through which it will generate a "trapped vortex" inside it, thus redistributing the pressure around the modified airfoil surfaces. The novelty of the work is both in the applications of such passive control technique as well as carrying out the investigations at transonic and fluctuating freestream conditions. Various test cases were examined, and the main conclusions are listed below.

\subsubsection{Transonic Flow Aerodynamic Improvement}

Thirty-two different STVG geometries were proposed and examined via CFD for a NACA 0012 airfoil as well as a NASA SC(3)-0712(B) supercritical airfoil. The study showed that the upper surface modifications were able to reduce the strength of the shockwave more effectively than the lower surface ones, yielding a significant drag reduction. 
For the NACA 0012 airfoil, the best upper surface modifications were able to reduce drag by $20 \%$, at only minor deduction in lift. Moreover, the $2^{\text {nd }}$ and $3^{\text {rd }}$ generation upper surface modification allowed to increase the lift-to drag ratio by as much as $32 \%$.

On the other hand, the lower surface modifications have the ability to double the lift with the majority of the designs and also exhibiting a sensible reduction in drag (up to $12 \%$ ) but with a penalty in pitching moment. Yet, they have the ability to increase the lift-to-drag ratio by a maximum of $44 \%$.

For the NASA SC(3)-0712(B) supercritical airfoil, the drag reduction was better if not the same when compared to the symmetric airfoil, while the lift-to-drag ratio was in the same order of magnitude. The maximum reduction in drag was about $35 \%$ while the lift-to-drag ratio increase about $34 \%$.

It is also worth to mention that in general, all 32 proposed modifications proved to be suitable for implementation on any symmetrical and cambered airfoils. However, for modern supercritical airfoils - due to their unique design features, one must carefully select the STVG type that leads to maximum gain at minimum surface modifications.

The novel airfoil modifications $\left(2^{\text {nd }}, 3^{\text {rd }}\right.$ and $4^{\text {th }}$ generations $)$ proved to be very good alternatives to the stepped airfoil modifications at high flight speeds. They demonstrated very good aerodynamic characteristics not only for a symmetric NACA 0012 airfoil, but also for a realistic supercritical airfoil, promising a variety of applications in the future. For fixed-wing aircraft, since the flight range is proportional to the lift-to-drag ratio, any increase in this ratio will allow for longer flights and less fuel consumption during the cruise segment of the flight. There is great potential to improve these features by using the proposed novel airfoil modifications. For helicopters, the 
results presented in this study could be promising for the advancing blades and could lead to decrease in required power or increase in thrust.

The physical explanation behind drag reduction and lift increase was the ability of the STVG to modify the location of the shock wave: it moved it to a more aft position. This meant that more area (before the shock wave) was exposed to low pressure supersonic flow, while less area (after the shock wave) to flow separation. Moreover, it is also suspected that the STVG changes the flow characteristics over the airfoil either by altering the circulation or by thickening the boundary layer.

\subsubsection{Constant Freestream Dynamic Stall Mitigation}

The advantage of using the proposed STVG in a dynamic stall environment is that, it affects the airfoil characteristics only close to the maximum angle of attack range, while largely maintaining the airfoil's original behavior at the lower angles of attack. Thus, although the system is passive, its functionality mimics the effects of an active flow control system tuned to reduce vibration (i.e. peak negative pitching moments) at those phases of the cycle, where dynamic stall occurs. This makes the concept a potential alternative for rotary wing flow control, where implementation of active flow control technologies is not trivial due to the large centripetal forces and limited space.

Twenty-six different STVG geometries were proposed and examined via CFD for the dynamic stall cases. The studies showed that the upper surface modifications were able to mitigate the negative effects of dynamic stall better than the lower surface ones.

The best upper surface modifications were able to reduce the peak negative pitching moment by about $50-60 \%$, peak drag by $30-40 \%$, while sacrificing only $2-10 \%$ of peak lift. It is also worth to draw the attention to the fact that drag reductions were 
noticed even at the irregularities near the maximum angle of attack. These results are remarkable and comparable to the effects of Active Flap Control, but without the complexity of implementing moving surfaces in a high-centripetal force dominated environment.

On the other hand, the lower surface modifications have the ability to increase the maximum lift by as much as $16 \%$ with minor penalty in pitching moment and drag.

The presented flow control concept is simple and could appear as "grooves" on the upper/lower surface of the blade at the blade span where dynamic stall occurs.

\subsubsection{Methodology to Investigate Shock-induced Dynamic Stall}

A methodology for simulating 2D shock-induced dynamic stall under realistic fluctuating freestream was also described in this thesis. This work is among the first ones to provide a detailed methodology and validation for such case. In the current study, it was found that for both of Constant Freestream (CFS) and Fluctuating Freestream (FFS), the lift, drag and pitching moment should all be scaled by the mean velocity and density over one revolution. This approach is considered to be more suitable than scaling by the instantaneous values, since the resulting coefficients are in this way consistent with the unscaled forces and moment.

It was shown that dynamic stall under FFS significantly differs from the typical dynamic stall at CFS in many ways. Most comprehensive rotor codes are based on using dynamic stall data neglecting the fluctuating freestream and these - based on the results of this thesis - represent a rather conservative approach since these over-predict the loads when compared to that seen in dynamic stall under FFS. In general, FFS is characterized by shock-induced flow separation and as such, stall will occur much earlier than in 
constant freestream dynamic stall. Also, one can notice that the phenomena on the advancing blades do affect the phenomena on the retreating blades, emphasizing the need to consider fluctuating freestream when transonic flow is achieved on the advancing blades of a helicopter.

The effect of compressibility is significant when simulating dynamic stall under CFS conditions as this will shift the location of the peak aerodynamic loads to occur before the airfoil static-stall angle of attack, which decreases the overall values of the aerodynamic loads.

\subsubsection{Shock-induced Dynamic Stall Mitigation}

As previously discussed, during shock-induced dynamic stall, the blade will be subject to a transonic flow on the advancing side of the rotor disk and dynamic stall on the retreating side of the rotor disk. The two phenomena alternate each other at very high frequency. The benefit of the STVG here is that, it affects the airfoil characteristics favorably at both the transonic and dynamic stall phases.

Twenty different geometries were examined via CFD for the FFS case. The results showed that both the upper and lower surface modifications were able to mitigate the negative effects of shock-induced dynamic stall. Besides providing benefits in the peaks of the aerodynamic loops, the average of aerodynamic loads per one rotor cycle was also examined as a mean to assess the performance of the modified airfoil with passive flow control. Per one cycle, the average values of lift-to-drag ratio increased by $3-28 \%$, drag decreased by $11-19 \%$ and pitching moment decreased by $3-70 \%$, at virtually no penalty in lift. These results are important for designers (i.e. blade performance analysis and pitch-link structural design) and are comparable to the effects of active flow 
control concepts, but without the complexity of implementing moving surfaces in a highcentripetal force dominated environment.

The proposed flow control concept is simple and could be implemented in the form of "grooves" on the upper or lower surface of the blade.

\subsection{Thesis Contributions}

The foremost contributions of the thesis to contemporary research are:

1. The use of Fertis and Smith airfoil "stepped airfoil" $[19,20]$, which itself was inspired from Kline-Fogleman's airfoil [14, 17], has not been examined previously for the flow control of high Mach and Reynolds numbers flows, such as, of helicopter dynamic stall during forward flight, and transonic cruise of commercial jet transport. Therefore, this is the first work to examine the use of Fertis and Smith airfoil for mitigating the negative effects of transonic flows and dynamic stall.

2. Based on careful systematic studies and meticulous partial optimization studies, thirty-two novel different STVG geometries developed for generating trapped vortices in the current thesis. The geometries were designed to be implemented as a passive flow control on any type of airfoils. The implementation could occur on the airfoil's upper or lower surfaces, as well as the leading and trailing edges of the airfoil. No prior work in the literature has presented such unique shapes and designs for passive flow control of transonic flow and dynamic stall. 
3. The thesis provides a systematic methodology for conducting dynamic stall simulations at constant freestream. Notice that: the currently available data in the literature differ greatly in terms of the numerical parameters used, giving vague guidance to other researchers as to what should be the correct choice of these parameters. A comprehensive overview of existing simulation methodologies is provided, i.e. the best practices (i.e. what works and what not) on how to set up dynamic stall simulations, using a dynamic mesh or domain oscillation, providing useful guideline for other researchers and engineers, intending to perform dynamic stall simulations.

4. The thesis is the first one to provide a methodology to accurately simulating shock-induced dynamic stall (FFS dynamic stall). Moreover, the thesis is the first study to provide a set of careful verification tests for FFS as well as the first one to validate FFS simulations at the extremes of the Mach number envelope (to the knowledge of the author, these have not been done before by any other researcher).

5. Although there are several CFD papers in the literature that address dynamic stall under FFS conditions, yet, none of them aimed to investigate whether transonic conditions on the advancing blades can alter the dynamic stall behavior on the retreating blades. This thesis considers real-life UH-60 flight test data to investigate whether such interference between the two phenomenon is important or not. Hence, it provides a detailed comparison and discussions to the results obtained from the typical incompressible CFS dynamic stall, the compressible 
CFS dynamic stall, and the compressible FFS dynamic stall. The results clearly suggest that the FFS alters the dynamic stall mechanism documented for CFS in a major way, emphasizing that inclusion of this effect in the prediction of dynamic stall related rotor loads is imperative for rotor performance analysis and blade design. To the knowledge of the author, no other work has investigated the interference of transonic flow and dynamic stall within one cycle, and as such this thesis presents novel and original results in this field.

6. The thesis provides a recommendation (with proof) on how the aerodynamic forces and moments should be scaled properly in the FFS dynamic stall case. This was not addressed in prior literature and hence, prior FFS result may give false impression about how the FFS and CFS loads relate to each other in reality, i.e. in dimensional terms. To avoid this, it is proposed in this thesis that the mean values of FFS velocities and densities are used to no-dimensionalized the aerodynamic forces and moments, as this would yield trends consistent with the actual loads.

7. The thesis proposes a novel way of assessing the performance of rotorcraft blade airfoils, which design might aim to mitigate the negative effects of dynamic stall. The thesis proposes calculating the average load per one rotor cycle for evaluating the airfoil's performance.

8. Although the feasibility of several active control methods has been researched in the literature, implementing an active flow control system in a high-speed environment or in the rotating frame (i.e. on the helicopter blades, in which high- 
centripetal forces dominate the environment) is very challenging. Thus, the proposed novel flow control technique (STVGs) provide an alternative simple, reliable, airfoil-based control system, which could be more attractive for both transport airplane and helicopter developers, than active systems.

\subsection{Future Work}

Future work shall involve more detailed analysis of the flow mechanism, investigation of the effectiveness of the concepts (STVGs) at other flow conditions, as well as examining their feasibility in $3 \mathrm{D}$ rotor aeromechanic simulations and actual aircraft wings.

It is also important to conduct experimental studies to validate the conclusions of this thesis further. Setting-up an experimental model capable of reproducing shockinduced dynamic stall at the required amplitudes of freestream fluctuation has so far not been achieved in a wind tunnel and might be extremely challenging. Therefore, it might be necessary to validate the CFD results in a rotating test rig or flight tests.

Modelling the STVGs on a full set of helicopter rotor blades is an interesting subject to investigate in the future to understand how the presence of such a passive flow technique would affect the "blade-vortex interaction" phenomenon. Furthermore, aeroelasticity, vibration and aeronautics studies might be also strong research topics for the future to further expand the understanding on how the STVG would affect the performance of the main rotor. 


\section{References}

[1] Leishman, J. G., Principles of Helicopter Aerodynamics, $2^{\text {nd }}$ ed., Cambridge University Press, New York, 2006, Chapter 9.

[2] Lin, J. C., Howard, F. G., Bushnell, D. M. and Selby, G. V., "Investigation of Several Passive and Active Methods for Turbulent Flow Separation Control," $21^{\text {st }}$ Fluid Dynamics, Plasma Dynamics and Lasers Conference Seattle, AIAA, WA, U.S.A, 1990. doi: $10.2514 / 6.1990-1598$

[3] Reuss, R. L., Hoffman, M. J. \& Gregorek G. M., "Effects of Surface Roughness and Vortex Generators on The NACA 4415 Airfoil," National laboratory of the U.S. Department of Energy, Contract No. DE-AC36-83CH10093, 1995.

[4] Paul, K. C., Separation of flow, $1^{\text {st }}$ ed., Pergamon Press, Hanguy, 1970.

[5] Kim, J., Kline, S. J. \& Johnston, J. P., "Investigation of a reattaching turbulent shear layer: Flow over a backward facing step." Transactions of ASME, 102, 1980.

[6] Shih, C. and Ho, C. M., "Three dimensional recirculation flow in a backward facing step." Transaction of ASME, 116, 1994.

[7] Rygg, K., Alendal, G., \& Haugan, P. M., "Flow over a rounded backward-facing step, using a z-coordinate model and a $\sigma$-coordinate model." Ocean Dynamics, 61, 2011, pp. 1681-1696. 
[8] Yang, D., Li, J., Fan, Z., and Yao, D., “Aerodynamic Characteristics of Transonic and Supersonic Flow Over Rectangular Cavities," Flow Turbulence Combust, Vol. 84, 2010, pp. 639-652.

doi: 10.1007/s10494-010-9246-7

[9] Yang, J. T., Tsai, B. B. \& Tsai, G. L., "Separated reattaching flow over a backward facing step with uniform normal mass bleed." Journal of Fluids Engineering, Vol. 116. No. 29, 1994.

[10] Chun, K. B. \& Sung, H. J., "Control of turbulent separated flow over a backwardfacing step by local forcing." Experiments in Fluids, 21, 1996, pp. 417-426.

[11] Heenan, A.F. \& Morrison, J.F., "Passive control of back step flow." Experimental Thermal and Fluid Science, 16, 1998, pp. 122-132.

[12] Khan, O. U. \& Hoffmann, K. A, "Unsteady supersonic flows over a backward-facing step with applied magnetic field.” Journal of Spacecraft and Rockets, Vol. 47, No. 3,2010 .

[13] Jürgens, W., \& Kaltenbach, H. J., "The effect of sweep on the forced transitional flow over a backward-facing step.” Journal of Computers \& Fluids, 59, 2012, pp. 1-10.

[14] Kline, R. L., and Fogleman, F. F., U.S. Patent Application for a "Airfoil for Aircraft," Appl. No. 20195, filed 17 Mar. 1970.

[15] Lumsdaine, E., Johnson, Y. S., Fletcher, L. M., \& Peach, J. E., "Investigation of the Kline-Fogleman airfoil section for applications.” NASA, AE-74-1054-1, 1974. 
[16] DeLaurier, J. D., \& Harris, J. M., “An expermental investigation of the aerodynamic charactarastics of stepped-wedge airfoils at low speeds." Journal of American Institute of Aeronautics and Astronautics, 1974, pp. 68-74.

[17] Kline, R. L., and Fogleman, F. F., U.S. Patent Application for a "Airfoil for aircraft having improved lift generating device,” Appl. No. 4046338, filed 14 Oct. 1975.

[18] Dave Powers, KF Airfoils: explained by Dave Powers. [Online]. Available: https://www.rcpowers.com/community/threads/kf-airfoils-explained-by-davepowers.2445/ [Accessed: 30 Nov. 2018].

[19] Fertis, D. G., \& Smith, L. L., U.S. Patent Application for a “Airfoil," Appl. No. 4606519, filed Aug. 6, 1984.

[20] Fertis, D. G., "New Airfoil Design Concept with Improved Aerodynamic Characteristics," Journal of Aerospace Engineering, Vol. 7, No. 3, 1994, pp. 328339.

[21] Finaish, F. and Witherspoon, S., "Aerodynamic Performance of an Airfoil with Step Induced Vortex for Lift Augmentation,” Journal of Aerospace Engineering, Vol. 11, No. 1, 1998, pp. 9-16.

[22] Al-Jaburi, K. J., “An Experimental Study of Backward Facing Step's Effect on The Aerodynamic Characteristics of Airfoils at Low Flight Speeds," Master's Thesis, University of Technology, Baghdad, 2003.

[23] Voona, R., "Enhancing the aerodynamic performance of the stepped airfoils." Master's Thesis, Missouri University of Science and Technology. Missouri, 2012. 
[24] Boroomand, M., Hosseinverdi, S., "Numerical Investigation of Turbulent Flow Around a Stepped Airfoil at High Reynolds Number," Proceedings of the ASME 2009 Fluids Engineering Division Summer Meeting, Vail, Colorado, August 2009.

[25] Mishriky, F., and Walsh, P., "Effect of the Backward-Facing Step Location on the Aerodynamics of a Morphing Wing," MDPI, Aerospace, Vol. 3, No. 25, 2016, pp. 1-15.

doi:10.3390/aerospace3030025

[26] Zha, G. C. and Paxton, C., “A Novel Airfoil Circulation Augment Flow Control Method Using Co-Flow Jet," $2^{\text {nd }}$ AIAA Flow Control Conference, AIAA, Portland, Oregon, 2004, pp. 1-13.

doi: $10.2514 / 6.2004-2208$

[27] You, D. and Moin, P., "Large-Eddy Simulation of Flow Separation Over an Airfoil with Synthetic Jet Control," Center for Turbulence Research Annual Research Briefs, 2006, pp. 337-346.

[28] Yeung, W.W.H., "Lift Enhancement on Unconventional Airfoils," Journal Mekanikal, No. 22, Dec. 2006, pp. 17-25.

[29] Shan, H., Jiang, L., Liu, C., Love M., and Maines, B., "Numerical Study of Passive and Active Flow Separation Control over a NACA0012 Airfoil," Computers \& Fluids, No. 37, 2008, pp. 975-992.

[30] Prince, S. A., Khodagolian, V., and Singh, C., “Aerodynamic Stall Suppression on Airfoil Sections Using Passive Air-Jet Vortex Generators," AIAA Journal, Vol. 47, No. 9, 2009, pp. 2232-2242. 
[31] Anand, U., Sudhakar, Y., Thileepanragu, R., Gopinathan, V.T., and Rajasekar, R., "Passive Flow Control over NACA0012 Airfoil Using Vortex Generators," Proceedings of the 37th National \& 4th International Conference on Fluid Mechanics and Fluid Power, IIT Madras, Chennai, India, Dec. 16-18, 2010, pp. $1-8$.

[32] Favier, J., Pinelli, A., \& Piomelli, U., "Control of The Separated Flow Around an Airfoil Using a Wavy Leading Edge Inspired by Humpback Whale Flippers," Comptes Rendus Mecanique, Elsevier, Vol. 340, No. (1-2), 2012, pp. 107-114.

[33] Srivastav, D., "Flow Control over Airfoils Using Different Shaped Dimples," International Conference on Fluid Dynamics and Thermodynamics Technologies, Vol. 33, 2012.

[34] Yagiz B., Kandil O., and Pehlivanoglu, Y. V., "Drag Minimization Using Active and Passive Flow Control Techniques," Aerospace Science and Technology, Elsevier, Vo. 17, 2012, pp. 21-31. doi: 10.1016/j.ast.2011.03.003

[35] Bourgois, S., Tensi, J., Sommier E., and Favier, J., "Experimental Investigation on Fluidic Control over an Airfoil," Journal of Flow Visualization and Image Processing, Begell House, Vol.13, No. 3, 2014, pp. 265-286.

[36] Bouzaher, M. T., "Large Eddy Simulation of Flow Separation Control over a NACA2415 Airfoil," International Journal of Mechanical, Aerospace, Industrial and Mechatronics Engineering, Vol. 8, No. 5, 2014, pp. 867-870. 
[37] Singh, C., Peake, D. J., Kokkalis, A. and Khodagolian, V., "Control of Rotorcraft Retreating Blade Stall Using Air-Jet Vortex Generators," Journal of Aircraft, Vol. 43, No. 4, 2006, pp. 1169-1176.

[38] Roedts II, R. L., "Rotorcraft Performance Enhancement Due to a Lower-Surface Miniature Effector," Masters Theses, Dept. of Aerospace Engineering, The Pennsylvania State Univ., United States, 2008.

[39] Barbarino, S., Gandhi, F. and Webster, S. D., "Design of Extendable Chord Sections for Morphing Helicopter Rotor Blades," Journal of Intelligent Material Systems and Structures, Vol. 22, June 2011.

doi: $10.1177 / 1045389 X 11414077$

[40] Rong, W. and PinQi. X., "Control of Dynamic Stall of Helicopter Rotor Blades,” Sci China Tech Sci, Vol. 56, No. 1, 2012, pp. 171-180.

doi: $10.1007 /$ s11431-012-5022-3

[41] Heine, B., Mulleners, K., Joubert, G. and Raffel, M., "Dynamic Stall Control by Passive Disturbance Generators," 29 $9^{\text {th }}$ AIAA Applied Aerodynamics Conference, AIAA, Honolulu, Hawaii, 2011. pp. 1-14.

doi: $10.2514 / 6.2011-3371$

[42] Le Pape, A., Lienard, C., and Bailly, J., "Active Flow Controls of Helicopters," Journal of Aerospace Lab, Vol. 6, No. 4, 2013, pp. 1-11.

[43] Vu, N. A., Lee, J. W., and Shu, J. I., “Aerodynamic Design Optimization of Helicopter Rotor Blades Including Airfoil Shape for Hover Performance,” Chinese Journal of Aeronautics, Vol. 26, No. 1, 2013, pp. 1-8. 
doi: 10.1016/j.cja.2012.12.008

[44] Lefebvre, A. and Zha, G. C., "Pitching Airfoil Performance Enhancement Using CoFlow Jet Flow Control at High Mach Number," $52^{\text {nd }}$ Aerospace Sciences Meeting, AIAA SciTech, Maryland, 2014, pp. 1-16.

[45] Cesnik, C. E. S., Opoku, D. G., Nitzsche, F., and Cheng, T. "Active twist rotor blade modelling using particle-wake aerodynamics and geometrically exact beam structural dynamics," Journal of Fluids and Structures, Vol. 19, 2004, pp. 651-668.

[46] Wilbur, M. L., Mirick, P. H., Yeager Jr, W. T., Langston, C. W., Cesnik, C. E. S., and Shin, S. "Vibratory loads reduction testing of the NASA/Army/MIT active twist rotor," Journal of the American Helicopter Society, Vol. 47, 2002, pp. 123-133.

[47] Mander, A., Feszty, D., and Nitzsche, F. "Active pitch link actuator for impedance control of helicopter vibration," 64th Annual Forum of the American Helicopter Society, Montreal, QC, 2008.

[48] Richardson, M., Feszty, D., A. P. J., Nitzsche, F., Khomutov, K. V., Vamosi, S. B., and Young, R. D. "Towards closed-loop control tests of the SHARCS active pitch link in the rotating frame," 67th Annual Forum of the American Helicopter Society, Virginia Beach, VA, 2011.

[49] Kloeppel, V. and Enenkl, B. "Rotor blade control by active helicopter servo flaps," International Forum on Aeroeleasticty and Structural Dynamics, Munich, Germany, 2005.

[50] Gardner, A.D., Knopp, T., Richter, K., Rosemann, H., "Numerical investigation of pulsed air jets for dynamic stall control on the OA209 airfoil", In New Results in 
Numerical and Experimental Fluid Mechanics VIII, Contributions to the 17th STAB/DGLR Symposium Berlin, Germany 2010, Vol. 121, of the series Notes on Numerical Fluid Mechanics and Multidisciplinary Design, Springer-Verlag, pp. 287-295, 2013.

[51] Anusonti-Inthra, P., Gandhi, F. "Helicopter vibration reduction through cyclic variations in rotor blade root stiffness", Journal of Intelligent Material Systems and Structures, Vol. 11, (2), 2000, pp. 153-166.

[52] Heverly D. E., Wang, K. W., Smith, and Edward, C., “An optimal actuator placement methodology for active control of helicopter airframe vibration," Journal of the American Helicopter Society, Vol. 46 (4), 2001, pp. 251-261(11).

[53] Konstanzer, P., Enenkl, B., Aubourg, P., Cranga, P., “Recent advances in Eurocopter's passive and active vibration control," 64th Annual Forum of the American Helicopter Society, Montreal, QC, Canada, 2008.

[54] Gardner, A. D., Opitz, S., Wolf, C. C., and Merz, C. B., "Experiment demonstrating reduction of dynamic stall by a back-flow flap," 72nd Annual Forum of the American Helicopter Society, Florida, May 2016.

[55] McCroskey, W. J. and Pucci, S. L., "Viscous-Inviscid Interaction on Oscillating Airfoils in Subsonic Flow," AIAA Journal, Vol. 20, (2), 1982, pp. 167-174.

[56] Carr, L. W., McAlister, K. W., and McCroskey, W. J., "Analysis of the Development of Dynamic Stall Based on Oscillating Airfoil Experiments," NASA TN D-8382, 1977. 
[57] Lee, T. and Gerontakos, P., "Investigation of flow over an oscillating airfoil," Journal of Fluid Mechanics, Vol. 512, 2004, pp. 313-341.

[58] Gardner, A. D., Richter, K., Mai, H., and Neuhaus, D., "Experimental investigation of air jets to control shock induced dynamic stall," Journal of the American Helicopter Society, Vol. 59, (2), 2014, pp. 022003-1-11.

[59] McAlister, K. W., Pucci, S. L., McCroskey, W. J., and Carr, L.W., “An Experimental Study of Dynamic Stall on Advanced Airfoil Sections, Volume 2. Pressure and Force Data,” NASA TM 84245, 1982.

[60] Gosselin, D. and Feszty, D., "Numerical investigation of the effect of shock-induced flow separation on dynamic stall in time-varying freestream conditions," $72^{\text {nd }}$ American Helicopter Society International Annual Forum, West Palm Beach, Florida, May 2016.

[61] Pierce, G. A., Kunz, D. L., and Malone, J. B., "Effect of varying freestream velocity on airfoil dynamic stall characteristics," Journal of the American Helicopter Society, vol. 23, no. 2, pp. 27-33, 1978.

[62] Maresca, C. A., Favier, D. J., and Rebont, J. M., "Unsteady Aerodynamics of An Aerofoil At High Angle of Incidence Performing Various Linear Oscillations in A Uniform Stream," Journal of the American Helicopter Society, vol. 26, no. 2, pp. 40-45, 1981.

[63] Brendel, M. and Mueller, T. J., "Features of Transitional Separation Bubbles in an Oscillating Freestream", Forum on Unsteady Flow Separation," in ASME Applied 
Mechanics, Bioengineering and Fluids Engineering Conference, Cincinnati, Ohio, 1987.

[64] Favier, D., Agnes, A., Barbi, C., and Maresca, C., "Combined Translation/Pitch Motion: A New Airfoil Dynamic Stall Simulation," Journal of Aircraft, vol. 25, no. 9, pp. 805-814, 1988.

[65] Ellsworth, R. H., and Mueller, T. J., "Airfoil boundary layer measurements at low Re in an accelerating flow from a nonzero velocity," Experiments in Fluids, vol. 11, no. 6 , pp. 368-374, 1991.

[66] Favier, D., Belleudy, J. and Maresca, C., "Influence of Coupling Incidence and Velocity Variations on the Airfoil Dynamic Stall," in AHS $48^{\text {th }}$ Annual Forum, Washington DC, June 3-5, 1992.73.

[67] Shi, Z. and Ming, X., "Effects of Unsteady Freestream on Aerodynamic Characteristics of Pitching Delta Wing," Journal of Aircraft, vol. 45, no. 11, pp. 2182-2185, 2008.

[68] Shi, Z. and Ming, X., "Experimental Investigation on a Pitching Motion Delta Wing in Unsteady Freestream," Modern Physics Letters B, vol. 23, no. 3, pp. 409-412.

[69] Gompertz, K., Jensen, C. D., Gregory, J. W. and Bons, J. P., "Compressible dynamic stall mechanisms due to airfoil pitching and freestream Mach oscillations," in $68^{\text {th }}$ AHS International Annual Forum, Fort Worth, TX, May 1-3, 2012.

[70] Kerstens, W. and Williams, D. R., "Energy exchange measurements with a longitudinally oscillating flow and a vertically oscillating wing," in $50^{\text {th }}$ AIAA 
Aerospace Sciences Meeting including the New Horizons Forum and Aerospace Exposition, Nashville, TN, 2012.

[71] Kerho, M. "Adaptive Airfoil Dynamic Stall Control," Journal of Aircraft, vol. 44, no. 4, pp. 1350-1360, 2007.

[72] Martinat, G., Braza, M., Harran, G., Sevrain, A., Tzabiras, G., Hoarau Y. and Favier, D., "Dynamic Stall of a Pitching and Horizontally Oscillating Airfoil," in IUTAM Symposium on Unsteady Separated Flows and their Control, 2009.

[73] Gharali, K. and Johnson, D. A., "Dynamic stall simulation of a pitching airfoil under unsteady freestream velocity," Journal of Fluids and Structures, Vol. 42, 2013, pp. $228-244$.

[74] Glaz, B., Liu, L., and Friedmann, P. P., "Reduced- Order Nonlinear Unsteady Aerodynamic Modeling Using a Surrogate-Based Recurrence Framework," AIAA Journal, Vol. 48, (10), October 2010, pp. 2418-2429.

[75] Glaz, B., Liu, L., Friedmann, P. P., Bain, J., and Sankar, L. N., “A Surrogate Based Approach to Reduced-Order Dynamic Stall Modeling,” Paper AIAA 2010-3042, $51^{\text {st }}$ AIAA/ASME/ASCE/AHS/ASC Structures, Structural Dynamics, and Materials Conference, Orlando, Florida, 12-15 April 2010.

[76] Fernie, R. M. and Babinsky, H., "Unsteady shock motion on a NACA0012 aerofoil at low reduced frequencies," Paper AIAA 2004-49, 42 ${ }^{\text {nd }}$ AIAA Aerospace Sciences Meeting and Exhibit, Reno, NV, January 2004.

[77] Hird, K., Frankhouser, M. W., Gregory, J. W., and Bons, J. P., "Compressible Dynamic Stall of an SSC-A09 Airfoil Subjected to Coupled Pitch and Freestream 
Mach Oscillations," Proceedings of the AHS $70^{\text {th }}$ Annual Forum, Montreal, Quebec, Canada, 20-22 May 2014.

[78] Hird, K., Frankhouser, M. W., Naigle, S., Gregory, J. W., and Bons, J. P., "Study of an SSC-A09 Airfoil in Compressible Dynamic Stall with Freestream Mach Oscillations," Proceedings of the AHS $71^{\text {st }}$ Annual Forum, Virginia Beach, Virginia, 5-7 May 2015.

[79] Zhao, Q., and Wang, Q. "Unsteady aerodynamic characteristics investigation of rotor airfoil under variational freestream velocity," Journal of Aerospace Science and Technology, vol. 58, pp. 82-91, 2016.

[80] “ANSYS FLUENT Theory Guide,” Canonsburg: ANSYS, Inc., 2016.

[81] “ANSYS FLUENT Solver Guide,” Canonsburg: ANSYS, Inc., 2016.

[82] Ko, S., and McCroskey, W. J., "Computations of Unsteady Separating Flow over an Oscillating Airfoil,” AIAA Journal, Vol. 35, (7), 1997, pp. 1235-1238.

[83] Ravindran, S., “Active Control of Flow Separation Over an Airfoil,” NASA TM-1999209838, Dec. 1999.

[84] Richter, K., Le Pape, A., Knopp, T., Costes, M., Gleize, V. and Gardner, A. D., “Improved Two-Dimensional Dynamic Stall Prediction with Structured and Hybrid Numerical Methods," 56th Annual Forum of the American Helicopter Society, Grapevine, TX, May 2009. 
[85] Richter, K., Koch, S., and Gardner, A. D., "Influence of oscillation amplitude and Mach number on the unsteady transition on a pitching rotor blade airfoil," $69^{\text {th }}$ Annual Forum of the American Helicopter Society, Phoenix, AZ, May 2013.

[86] Klein, A., Lutz, Th., and Krämer, E., "Numerical Comparison of Dynamic Stall for Two-Dimensional Airfoils and an Airfoil Model in the DNW-TWG," $37^{\text {th }}$ European Rotorcraft Forum, Gallarate, Italy, September 2011.

[87] Al-Jaburi, K. J., and Feszty, D., "Passive Flow Control of Dynamic Stall via Surfacebased Trapped Vortex Generators," Journal of the American Helicopter Society, Vol. 63, No. 3, 2018, pp. 1-14. doi: https://doi.org/10.4050/JAHS.63.032005

[88] Charles D. H., "Two-Dimensional Aerodynamic Characteristics of the NACA0012 Airfoil in The Langley 8-foot Transonic Pressure Tunnel," NASA TM-81927, 1981.

[89] Johnson W. G., Jr., Hill, A. S., and Eichmann O., "High Reynolds Number Tests of a NASA SC(3)-0712(B) Airfoil in the Langley 0.3-Meter Transonic Cryogenic Tunnel," NASA TM-86371, 1985.

[90] Thomas, J. L., and Salas, M. D., “Far-field Boundary Conditions for Transonic Lifting Solutions to The Euler Equations," AIAA Journal, Vol. 24, No. 7, 1986, pp. 10741080.

[91] Blazek, J., Computational Fluid Dynamics: Principles and Applications, $1^{\text {st }}$ ed., Elsevier Science Ltd, United Kingdom, 2001, Chapter 8. 
[92] Rumsey, C., Smith, B. R., and Huang, G. P., "Description of a Website Resource for Turbulence Modeling Verification and Validation," $40^{\text {th }}$ AIAA Fluid Dynamics Conference and Exhibit, Chicago, IL, 2010, pp. 1-17.

[93] McAlister, K. W., Pucci, S. L., McCroskey, W. J., and Carr, L.W., “An Experimental Study of Dynamic Stall on Advanced Airfoil Sections, Volume 2. Pressure and Force Data,"NASA TM 84245, 1982.

[94] “ANSYS FLUENT User’s Guide,” Canonsburg: ANSYS, Inc., 2016.

[95] Shinoda, P., M., Yeo, H., and Norman, T., R., "Rotor Performance of a UH-60 Rotor System in the NASA Ames 80 - by 120 -Foot Wind Tunnel," 58 ${ }^{\text {th }}$ American Helicopter Society International Annual Forum, Montreal, Canada, June 2002.

[96] Nagata, J., Skinner, G., Buckanin, R., Robbins, R., and Williams, R., “Airworthiness and Flight Characteristics Evaluation UH-60A (Black Hawk) Helicopter," USAAEFA Project No. 77-17, United states Army Aviation Engineering Flight Activity, Edwards air force base, California 93523, 1981.

[97] Buckanin, R., Kelly, W., Webre, J., Reynolds, T., Lockwood, R. and Cason, R., “Level Flight Performance Evaluation of the UH-60A Helicopter with the Production External Stores Support System and Ferry Tanks Installed,” USAAEFA Project No. 86-01, United states Army Aviation Engineering Flight Activity, Edwards air force base, California 93523, 1986.

[98] Murrell, R., Lockwood, R., Williams, R., Herbst, M., Skinner, G., and Cripps, D., "Flight Evaluation of the UH-60A Helicopter with the Pitch Bias Actuator Centered and Electricity Disconnected," USAAEFA Project No. 84-28, United states Army 
Aviation Engineering Flight Activity, Edwards air force base, California 93523, 1986.

[99] Coleman, C. P. and Bousman W., G., "Aerodynamic Limitations of the UH-60A Rotor,” NASA TM 110396, August 1996.

[100] Bousman W., G., "Airfoil Design and Rotorcraft Performance," 58 ${ }^{\text {th }}$ American Helicopter Society International Annual Forum, Montreal, Canada, June 2002.

[101] Destarac, D., "Spurious Far-Field-Boundary Induced Drag in Two-Dimensional Flow Simulations," Journal of Aircraft, Vol. 48, (4), 2011, pp. 1444-1455.

[102] Thompson, K. W., "Time- Dependent Boundary Conditions for Hyperbolic Systems," Journal of Computational Physics, Vol. 68, 1987, pp. 1-24.

[103] Thompson, K. W., “Time- Dependent Boundary Conditions for Hyperbolic Systems, II,” Journal of Computational Physics, Vol. 89, 1990, pp. 439-461.

[104] Flemming, R., J., “An Experimental Evaluation of Advanced Rotorcraft Airfoils in the NASA Ames Eleven-Foot Transonic Wind Tunnel," NASA Contract Report $166587,1984$.

[105] Lorber, P. F. and Carta, F. O., "Unsteady stall penetration experiments at high Reynolds number,” Technical report, DTIC Document, 1987.

[106] Gregory, J., W., “Unsteady Aerodynamics: Time-Varying Compressible Dynamic Stall Mechanisms Due to Freestream Mach Oscillations," U.S. Army Research Office Report 58835-EG-YIP.3, 2014.

[107] “ANSYS FLUENT Adjoint Solver,” Canonsburg: ANSYS, Inc., 2011. 
[108] Wakefield, I., and Dubuque, C., "Exceeding Tire Speed Rating During Takeoff," Boeing Aero-magazine, Aero quarterly QTR02, 2009.

[109] Gardner, A., D., Richter, K., Mai, H., and Neuhaus, D., "Experimental Investigation of Air Jets to Control Shock-Induced Dynamic Stall," Journal of the American Helicopter Society, vol. 59, pp. 1-11, 2014.

[110] Sangwan, J., Sengupta, T., K., and Suchandra, P., "Investigation of compressibility effects on dynamic stall of pitching airfoil," Journal of Physics of Fluids, vol. 29, pp. 1-15, 2017.

[111] Bousman W., G., "Aerodynamic Characteristics of SC1095 and SC1094 R8 Airfoils," NASA/TP-2003-212265, December 2003. 


\section{Appendix A. FLUENT Input Files}

This appendix comprises of two examples (A.1) and (A.2). The input files used to modify ANSYS FLUENT solver to accommodate dynamic stall simulations. The modification involves a User Define Functions (UDF) used to introduce the oscillation motion of the airfoil in the constant freestream dynamic stall simulations (A.1), and also to provide the fluctuation in the freestream at the domain inlet for shock-induced dynamic stall simulations (A.2). 


\section{A.1 UDF Example for Constant Freestream Dynamic Stall Simulation}

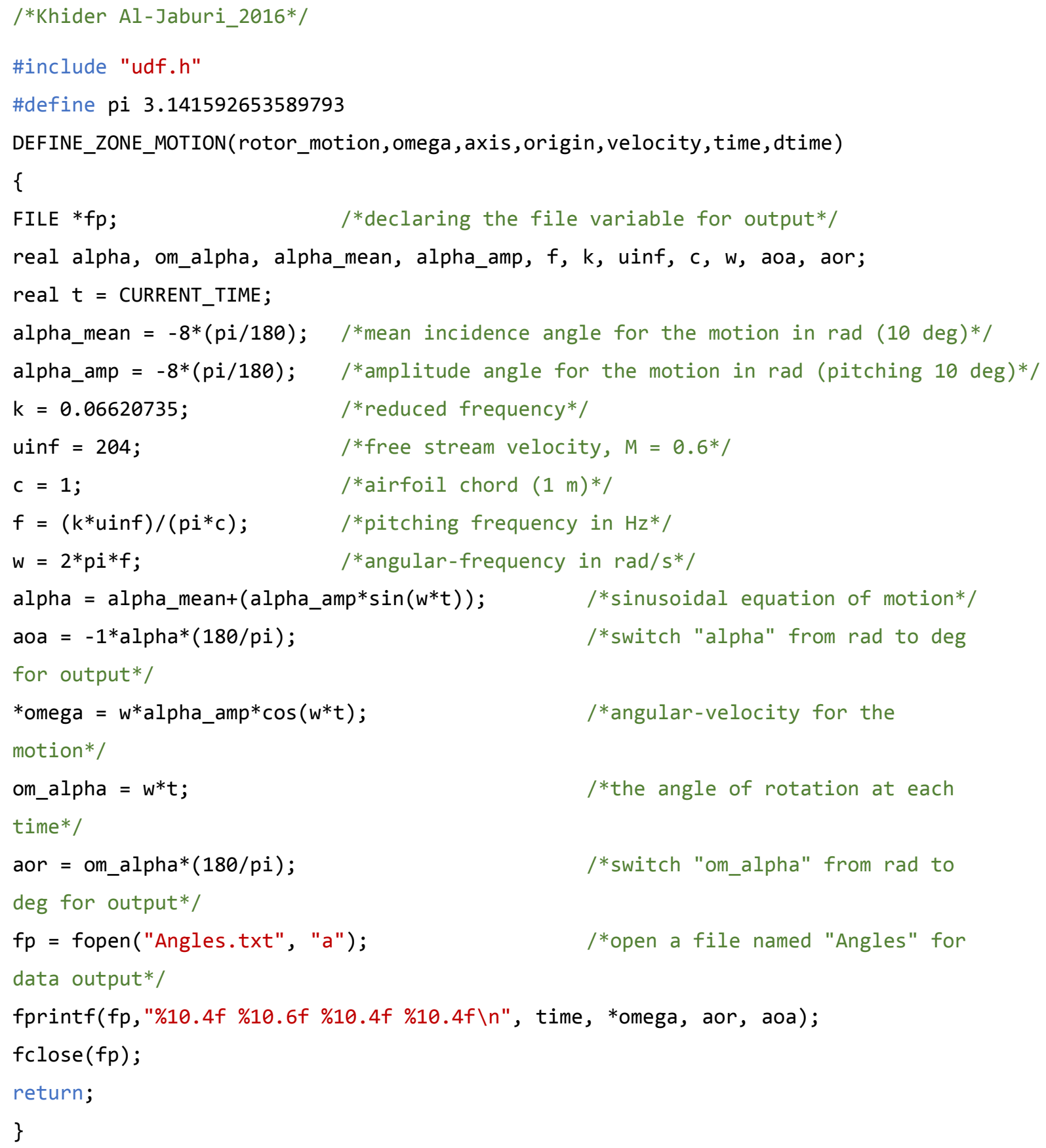




\section{A.2 UDF Example for Unsteady Freestream Dynamic Stall Simulation}

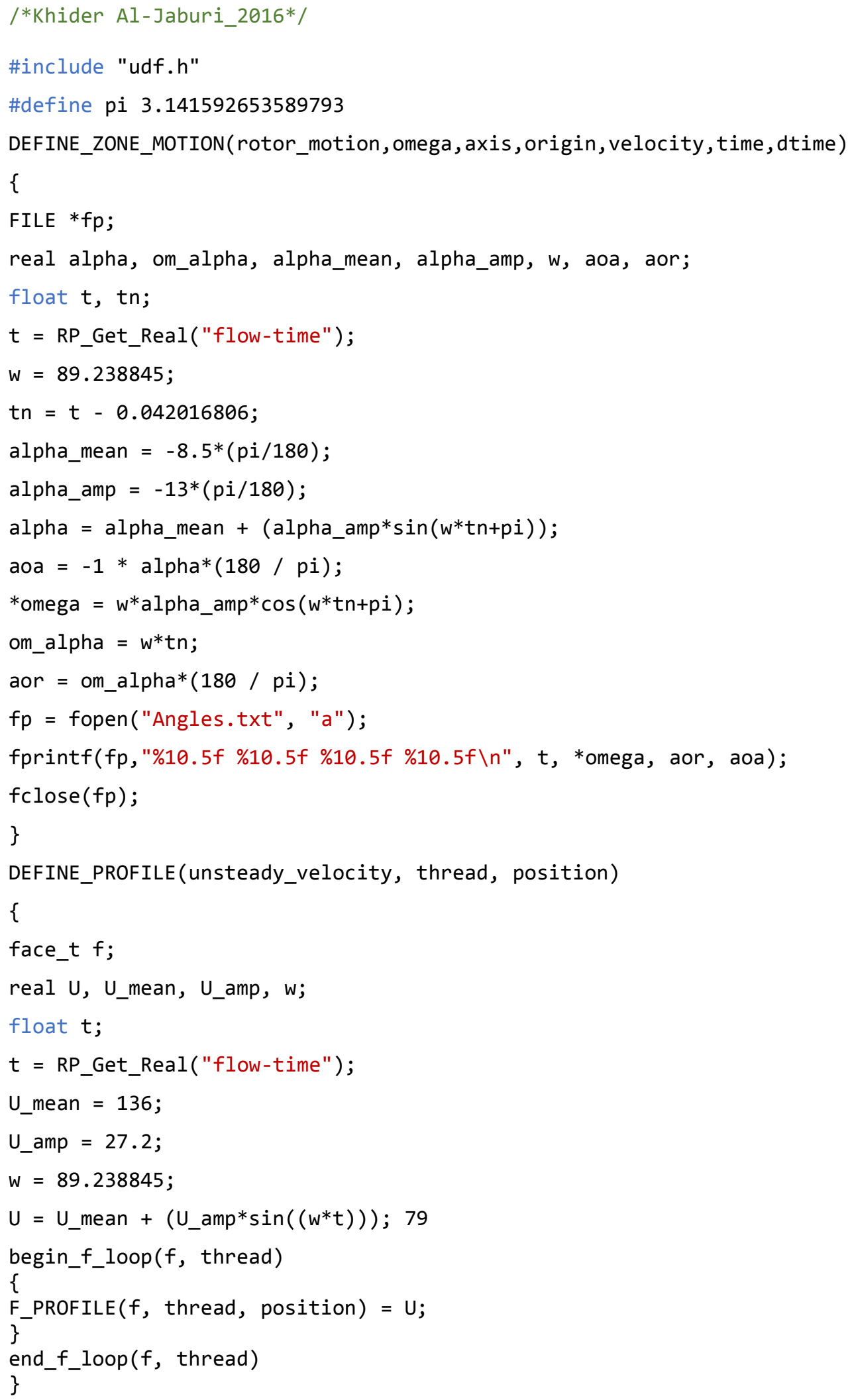




\section{Appendix B. Baseline Airfoils Geometries Coordinates}

This appendix provides the coordinates of the baseline airfoil geometries. The geometries consist of NACA 0012 airfoil and NASA SC(3)-0712(B) supercritical airfoil, used for the transonic flow simulations (B.1 and B.2), NACA 0012 airfoil used in constant freestream dynamic stall simulations (B.3). In addition, the airfoil coordinates of SSC A09 used in validating the fluctuating freestream methodology will also be provided here (B.4). Finally, in B.5, the coordinates of SC1095 transonic airfoil used for the compressible dynamic stall simulations (both constant and fluctuating freestreams). All of the provided coordinates were reproduced from the airfoils experiments reports. 


\section{B.1 NACA 0012 Airfoil Coordinates: Transonic Flow Simulations}

Table B.1 NACA 0012 Airfoil Coordinates [88]

\begin{tabular}{cccc}
\multicolumn{2}{c}{ Upper Surface } & \multicolumn{2}{c}{ Lower Surface } \\
X/C [-] & Y/C $[-]$ & X/C $[-]$ & Y/C $[-]$ \\
0 & 0 & 0 & 0 \\
0.002 & 0.0078 & 0.002 & -0.0078 \\
0.005 & 0.0122 & 0.005 & -0.0122 \\
0.01 & 0.017 & 0.01 & -0.017 \\
0.02 & 0.0236 & 0.02 & -0.0236 \\
0.03 & 0.0284 & 0.03 & -0.0284 \\
0.04 & 0.0323 & 0.04 & -0.0323 \\
0.05 & 0.0355 & 0.05 & -0.0355 \\
0.06 & 0.0384 & 0.06 & -0.0384 \\
0.08 & 0.0431 & 0.08 & -0.0431 \\
0.1 & 0.0468 & 0.1 & -0.0468 \\
0.12 & 0.0499 & 0.12 & -0.0499 \\
0.14 & 0.0524 & 0.14 & -0.0524 \\
0.16 & 0.0544 & 0.16 & -0.0544 \\
0.18 & 0.0561 & 0.18 & -0.0561 \\
0.2 & 0.0574 & 0.2 & -0.0574 \\
0.22 & 0.0584 & 0.22 & -0.0584 \\
0.24 & 0.0591 & 0.24 & -0.0591 \\
0.26 & 0.0596 & 0.26 & -0.0596 \\
0.28 & 0.0599 & 0.28 & -0.0599 \\
0.3 & 0.06 & 0.3 & -0.06 \\
0.32 & 0.0599 & 0.32 & -0.0599 \\
0.34 & 0.0597 & 0.34 & -0.0597 \\
0.36 & 0.0593 & 0.36 & -0.0593 \\
0.38 & 0.0587 & 0.38 & -0.0587 \\
0.4 & 0.058 & 0.4 & -0.058 \\
0.42 & 0.0572 & 0.42 & -0.0572 \\
0.44 & 0.0563 & 0.44 & -0.0563
\end{tabular}


Table B.1 NACA 0012 Airfoil Coordinates Continued [88]

\begin{tabular}{|c|c|c|c|}
\hline \multicolumn{2}{|c|}{ Upper Surface } & \multicolumn{2}{|c|}{ Lower Surface } \\
\hline$X / C[-]$ & $\mathrm{Y} / \mathrm{C}[-]$ & $\mathrm{X} / \mathrm{C}[-]$ & $\mathrm{Y} / \mathrm{C}[-]$ \\
\hline 0.46 & 0.0553 & 0.46 & -0.0553 \\
\hline 0.48 & 0.0542 & 0.48 & -0.0542 \\
\hline 0.5 & 0.0529 & 0.5 & -0.0529 \\
\hline 0.52 & 0.0516 & 0.52 & -0.0516 \\
\hline 0.54 & 0.0502 & 0.54 & -0.0502 \\
\hline 0.56 & 0.0488 & 0.56 & -0.0488 \\
\hline 0.58 & 0.0472 & 0.58 & -0.0472 \\
\hline 0.6 & 0.0456 & 0.6 & -0.0456 \\
\hline 0.62 & 0.044 & 0.62 & -0.044 \\
\hline 0.64 & 0.0422 & 0.64 & -0.0422 \\
\hline 0.66 & 0.0404 & 0.66 & -0.0404 \\
\hline 0.68 & 0.0386 & 0.68 & -0.0386 \\
\hline 0.7 & 0.0366 & 0.7 & -0.0366 \\
\hline 0.72 & 0.0347 & 0.72 & -0.0347 \\
\hline 0.74 & 0.0326 & 0.74 & -0.0326 \\
\hline 0.76 & 0.0306 & 0.76 & -0.0306 \\
\hline 0.78 & 0.0284 & 0.78 & -0.0284 \\
\hline 0.8 & 0.0262 & 0.8 & -0.0262 \\
\hline 0.82 & 0.024 & 0.82 & -0.024 \\
\hline 0.84 & 0.0217 & 0.84 & -0.0217 \\
\hline 0.86 & 0.0193 & 0.86 & -0.0193 \\
\hline 0.88 & 0.0169 & 0.88 & -0.0169 \\
\hline 0.9 & 0.0145 & 0.9 & -0.0145 \\
\hline 0.92 & 0.012 & 0.92 & -0.012 \\
\hline 0.94 & 0.0094 & 0.94 & -0.0094 \\
\hline 0.96 & 0.0067 & 0.96 & -0.0067 \\
\hline 0.98 & 0.004 & 0.98 & -0.004 \\
\hline 1 & 0.0013 & 1 & -0.0013 \\
\hline
\end{tabular}




\section{B.2 NASA SC(3)-0712(B) Supercritical Airfoil Coordinates: Transonic Flow Simulations}

Table B.2 NASA SC(3)-0712(B) supercritical Airfoil Coordinates [89]

\begin{tabular}{cccc}
\multicolumn{2}{c}{ Upper Surface } & \multicolumn{2}{c}{ Lower Surface } \\
X/C [-] & Y/C [-] & X/C [-] & Y/C [-] \\
0.002 & 0.0092 & 0.002 & -0.0051 \\
0.005 & 0.0141 & 0.005 & -0.0081 \\
0.01 & 0.019 & 0.01 & -0.0116 \\
0.02 & 0.0252 & 0.02 & -0.0165 \\
0.03 & 0.0294 & 0.03 & -0.0204 \\
0.04 & 0.0327 & 0.04 & -0.0238 \\
0.05 & 0.0354 & 0.05 & -0.0266 \\
0.07 & 0.0397 & 0.07 & -0.0316 \\
0.08 & 0.0415 & 0.08 & -0.0338 \\
0.1 & 0.0446 & 0.1 & -0.0377 \\
0.12 & 0.0471 & 0.12 & -0.0412 \\
0.15 & 0.0504 & 0.15 & -0.0458 \\
0.18 & 0.053 & 0.18 & -0.0498 \\
0.2 & 0.0544 & 0.2 & -0.0521 \\
0.22 & 0.0557 & 0.23 & -0.055 \\
0.25 & 0.0572 & 0.25 & -0.0566 \\
0.28 & 0.0584 & 0.28 & -0.0585 \\
0.3 & 0.059 & 0.3 & -0.0595 \\
0.33 & 0.0596 & 0.33 & -0.0605 \\
0.35 & 0.0599 & 0.35 & -0.0609 \\
0.37 & 0.0601 & 0.38 & -0.061 \\
0.4 & 0.0601 & 0.4 & -0.0608 \\
0.42 & 0.06 & 0.43 & -0.06 \\
0.45 & 0.0596 & 0.45 & -0.0591 \\
0.48 & 0.059 & 0.48 & -0.0573
\end{tabular}


Table B.2 NASA SC(3)-0712(B) supercritical Airfoil Coordinates Continued [89]

\begin{tabular}{|c|c|c|c|}
\hline \multicolumn{2}{|c|}{ Upper Surface } & \multicolumn{2}{|c|}{ Lower Surface } \\
\hline $\mathrm{X} / \mathrm{C}[-]$ & $\mathrm{Y} / \mathrm{C}[-]$ & $\mathrm{X} / \mathrm{C}[-]$ & $\mathrm{Y} / \mathrm{C}[-]$ \\
\hline 0.5 & 0.0584 & 0.5 & -0.0558 \\
\hline 0.53 & 0.0573 & 0.53 & -0.053 \\
\hline 0.55 & 0.0564 & 0.55 & -0.0509 \\
\hline 0.58 & 0.0549 & 0.58 & -0.0472 \\
\hline 0.6 & 0.0537 & 0.6 & -0.0446 \\
\hline 0.63 & 0.0516 & 0.62 & -0.0419 \\
\hline 0.65 & 0.05 & 0.65 & -0.0376 \\
\hline 0.67 & 0.0482 & 0.68 & -0.0331 \\
\hline 0.7 & 0.0451 & 0.7 & -0.0299 \\
\hline 0.73 & 0.0416 & 0.72 & -0.0267 \\
\hline 0.75 & 0.039 & 0.75 & -0.0221 \\
\hline 0.77 & 0.0362 & 0.77 & -0.0191 \\
\hline 0.8 & 0.0316 & 0.8 & -0.0149 \\
\hline 0.83 & 0.0266 & 0.82 & -0.0123 \\
\hline 0.85 & 0.023 & 0.85 & -0.0088 \\
\hline 0.87 & 0.0192 & 0.88 & -0.0059 \\
\hline 0.9 & 0.0131 & 0.9 & -0.0049 \\
\hline 0.92 & 0.0088 & 0.93 & -0.0055 \\
\hline 0.94 & 0.0042 & 0.95 & -0.0074 \\
\hline 0.95 & 0.0018 & 0.96 & -0.0088 \\
\hline 0.96 & -0.0007 & 0.97 & -0.0105 \\
\hline 0.97 & -0.0033 & 0.98 & -0.0126 \\
\hline 0.98 & -0.006 & 0.99 & -0.015 \\
\hline 0.99 & -0.0088 & 1 & -0.0177 \\
\hline 1 & -0.0117 & & \\
\hline
\end{tabular}




\section{B.3 NACA 0012 Airfoil Coordinates: Constant Dynamic Stall Simulations}

Table B.3 NACA 0012 Airfoil Coordinates [93]

\begin{tabular}{cccc}
\multicolumn{2}{c}{ Upper Surface } & \multicolumn{2}{c}{ Lower Surface } \\
X/C $[-]$ & $Y / C[-]$ & X/C $[-]$ & $Y / C[-]$ \\
0 & 0 & 0 & 0 \\
0.0005 & 0.00395 & 0.0005 & -0.00395 \\
0.001 & 0.00556 & 0.001 & -0.00556 \\
0.002 & 0.00781 & 0.002 & -0.00781 \\
0.0035 & 0.01027 & 0.0035 & -0.01027 \\
0.005 & 0.01221 & 0.005 & -0.01221 \\
0.0065 & 0.01386 & 0.0065 & -0.01386 \\
0.008 & 0.01531 & 0.008 & -0.01531 \\
0.01 & 0.01704 & 0.01 & -0.01704 \\
0.0125 & 0.01894 & 0.0125 & -0.01894 \\
0.016 & 0.02127 & 0.016 & -0.02127 \\
0.02 & 0.0236 & 0.02 & -0.0236 \\
0.025 & 0.02615 & 0.025 & -0.02615 \\
0.035 & 0.03043 & 0.035 & -0.03043 \\
0.05 & 0.03555 & 0.05 & -0.03555 \\
0.065 & 0.03966 & 0.065 & -0.03966 \\
0.08 & 0.04307 & 0.08 & -0.04307 \\
0.1 & 0.04683 & 0.1 & -0.04683 \\
0.125 & 0.05055 & 0.125 & -0.05055 \\
0.15 & 0.05345 & 0.15 & -0.05345
\end{tabular}


Table B.3 NACA 0012 Airfoil Coordinates Continued [93]

\begin{tabular}{cccc}
\multicolumn{2}{c}{ Upper Surface } & \multicolumn{2}{c}{ Lower Surface } \\
X/C [-] & $Y / C[-]$ & $X / C[-]$ & $Y / C[-]$ \\
0.2 & 0.05737 & 0.2 & -0.05737 \\
0.25 & 0.05941 & 0.25 & -0.05941 \\
0.3 & 0.06002 & 0.3 & -0.06002 \\
0.35 & 0.05949 & 0.35 & -0.05949 \\
0.4 & 0.05803 & 0.4 & -0.05803 \\
0.45 & 0.05581 & 0.45 & -0.05581 \\
0.5 & 0.05294 & 0.5 & -0.05294 \\
0.55 & 0.04952 & 0.55 & -0.04952 \\
0.6 & 0.04563 & 0.6 & -0.04563 \\
0.65 & 0.04132 & 0.65 & -0.04132 \\
0.7 & 0.03664 & 0.7 & -0.03664 \\
0.75 & 0.0316 & 0.75 & -0.0316 \\
0.8 & 0.02623 & 0.8 & -0.02623 \\
0.85 & 0.02053 & 0.85 & -0.02053 \\
0.9 & 0.01448 & 0.9 & -0.01448 \\
0.925 & 0.01132 & 0.925 & -0.01132 \\
0.95 & 0.00807 & 0.95 & -0.00807 \\
0.975 & 0.00472 & 0.975 & -0.00472 \\
0.99 & 0.00265 & 0.99 & -0.00265 \\
1 & 0.00126 & 1 & -0.00126
\end{tabular}




\section{B.4 SSC A09 Airfoil Coordinates: Validation of Fluctuating Freestream Dynamic Stall Simulations}

Table B.4 SSC A09 Airfoil Coordinates [106]

\begin{tabular}{|c|c|c|c|}
\hline \multicolumn{2}{|c|}{ Upper Surface } & \multicolumn{2}{|c|}{ Lower Surface } \\
\hline $\mathrm{X} / \mathrm{C}[-]$ & $\mathrm{Y} / \mathrm{C}[-]$ & $\mathrm{X} / \mathrm{C}[-]$ & $\mathrm{Y} / \mathrm{C}[-]$ \\
\hline 0 & 0 & 0 & 0 \\
\hline 0.0002 & 0.002 & 0.0002 & -0.00145 \\
\hline 0.0008 & 0.00395 & 0.0008 & -0.00287 \\
\hline 0.00199 & 0.00648 & 0.00199 & -0.00457 \\
\hline 0.00299 & 0.00803 & 0.00299 & -0.00545 \\
\hline 0.00449 & 0.00987 & 0.00449 & -0.00645 \\
\hline 0.00698 & 0.01239 & 0.00698 & -0.0077 \\
\hline 0.00997 & 0.01492 & 0.00997 & -0.00888 \\
\hline 0.01595 & 0.01908 & 0.01595 & -0.0107 \\
\hline 0.02193 & 0.0225 & 0.02193 & -0.01218 \\
\hline 0.02792 & 0.02545 & 0.02792 & -0.01345 \\
\hline 0.0339 & 0.02804 & 0.0339 & -0.01459 \\
\hline 0.03988 & 0.03037 & 0.03988 & -0.01563 \\
\hline 0.04586 & 0.03249 & 0.04586 & -0.01659 \\
\hline 0.05185 & 0.03445 & 0.05185 & -0.01749 \\
\hline 0.05783 & 0.03625 & 0.05783 & -0.01831 \\
\hline 0.0678 & 0.0389 & 0.0678 & -0.01957 \\
\hline 0.07777 & 0.04114 & 0.07777 & -0.02069 \\
\hline 0.08774 & 0.04302 & 0.08774 & -0.02171 \\
\hline 0.09771 & 0.04458 & 0.09771 & -0.02264 \\
\hline 0.11266 & 0.0465 & 0.11266 & -0.02391 \\
\hline 0.12762 & 0.04805 & 0.12762 & -0.02506 \\
\hline 0.14257 & 0.04935 & 0.14257 & -0.02612 \\
\hline 0.15753 & 0.05044 & 0.15753 & -0.0271 \\
\hline 0.17249 & 0.05139 & 0.17249 & -0.02801 \\
\hline 0.18744 & 0.05218 & 0.18744 & -0.02885 \\
\hline 0.2024 & 0.05286 & 0.2024 & -0.02963 \\
\hline 0.21735 & 0.05343 & 0.21735 & -0.03034 \\
\hline 0.23231 & 0.05391 & 0.23231 & -0.03099 \\
\hline 0.24726 & 0.05432 & 0.24726 & -0.03158 \\
\hline 0.27717 & 0.05496 & 0.27717 & -0.03259 \\
\hline 0.30708 & 0.05537 & 0.30708 & -0.0334 \\
\hline 0.33699 & 0.05556 & 0.33699 & -0.03401 \\
\hline
\end{tabular}


Table B.4 SSC A09 Airfoil Coordinates Continued [106]

\begin{tabular}{cccc}
\multicolumn{2}{c}{ Upper Surface } & \multicolumn{2}{c}{ Lower Surface } \\
X/C [-] & Y/C [-] & X/C [-] & $Y / C[-]$ \\
0.37687 & 0.05549 & 0.37687 & -0.03451 \\
0.41675 & 0.05504 & 0.41675 & -0.03464 \\
0.43669 & 0.05466 & 0.43669 & -0.03456 \\
0.45664 & 0.05418 & 0.45664 & -0.03438 \\
0.47658 & 0.0536 & 0.47658 & -0.03409 \\
0.49652 & 0.0529 & 0.49652 & -0.03368 \\
0.51646 & 0.05209 & 0.51646 & -0.03317 \\
0.5364 & 0.05118 & 0.5364 & -0.03253 \\
0.55634 & 0.05015 & 0.55634 & -0.03179 \\
0.57628 & 0.04901 & 0.57628 & -0.03095 \\
0.59622 & 0.04776 & 0.59622 & -0.03002 \\
0.61616 & 0.04638 & 0.61616 & -0.029 \\
0.6361 & 0.04488 & 0.6361 & -0.0279 \\
0.65604 & 0.04322 & 0.65604 & -0.02672 \\
0.67598 & 0.04139 & 0.67598 & -0.02545 \\
0.69592 & 0.03937 & 0.69592 & -0.02409 \\
0.71586 & 0.03714 & 0.71586 & -0.02264 \\
0.7358 & 0.03472 & 0.7358 & -0.02112 \\
0.75574 & 0.03214 & 0.75574 & -0.01954 \\
0.77568 & 0.02945 & 0.77568 & -0.01792 \\
0.79562 & 0.02668 & 0.79562 & -0.01627 \\
0.81556 & 0.02387 & 0.81556 & -0.01462 \\
0.8355 & 0.02101 & 0.8355 & -0.01296 \\
0.85544 & 0.01809 & 0.85544 & -0.01129 \\
0.87538 & 0.01509 & 0.87538 & -0.0096 \\
0.89532 & 0.01205 & 0.89532 & -0.00786 \\
0.91526 & 0.00905 & 0.91526 & -0.00608 \\
0.9352 & 0.00623 & 0.9352 & -0.00429 \\
0.95514 & 0.00385 & 0.95514 & -0.00261 \\
0.97508 & 0.00229 & 0.97508 & -0.00133 \\
0.98506 & 0.00199 & 0.98506 & -0.00099 \\
0.99503 & 0.00214 & 0.99503 & -0.00086 \\
1 & 0.00241 & 1 & -0.0008
\end{tabular}




\section{B.5 SC 1095 Transonic Airfoil Coordinates: Compressible Constant and Fluctuating Freestream Dynamic Stall Simulations}

Table B.5 SC 1095 Airfoil Coordinates [111]

\begin{tabular}{cccc}
\multicolumn{2}{c}{ Upper Surface } & \multicolumn{2}{c}{ Lower Surface } \\
X/C [-] & Y/C [-] & X/C [-] & Y/C [-] \\
1 & 0.00173 & 1 & -0.00173 \\
0.99644 & 0.00201 & 0.99644 & -0.00188 \\
0.98627 & 0.00281 & 0.98627 & -0.00229 \\
0.97611 & 0.0036 & 0.97611 & -0.00271 \\
0.96594 & 0.00443 & 0.96594 & -0.00313 \\
0.95577 & 0.0057 & 0.95577 & -0.00402 \\
0.9456 & 0.00695 & 0.9456 & -0.00489 \\
0.93544 & 0.00818 & 0.93544 & -0.00575 \\
0.92527 & 0.00939 & 0.92527 & -0.00659 \\
0.9151 & 0.0106 & 0.9151 & -0.00742 \\
0.89476 & 0.01297 & 0.89476 & -0.00908 \\
0.87443 & 0.01532 & 0.87443 & -0.01072 \\
0.85409 & 0.01764 & 0.85409 & -0.01236 \\
0.83376 & 0.01993 & 0.83376 & -0.01397 \\
0.81342 & 0.02218 & 0.81342 & -0.01557 \\
0.79309 & 0.02439 & 0.79309 & -0.01714 \\
0.77275 & 0.02655 & 0.77275 & -0.01868 \\
0.75242 & 0.02865 & 0.75242 & -0.02019 \\
0.73208 & 0.0307 & 0.73208 & -0.02166 \\
0.71174 & 0.03267 & 0.71174 & -0.02308 \\
0.69141 & 0.03458 & 0.69141 & -0.02445 \\
0.67107 & 0.0364 & 0.67107 & -0.02577 \\
0.65074 & 0.03815 & 0.65074 & -0.02703 \\
0.6304 & 0.03982 & 0.6304 & -0.02824 \\
0.61007 & 0.0414 & 0.61007 & -0.02938 \\
0.58973 & 0.04291 & 0.58973 & -0.03047 \\
0.5694 & 0.04434 & 0.5694 & -0.03149 \\
0.54906 & 0.04568 & 0.54906 & -0.03245 \\
0.52872 & 0.04694 & 0.52872 & -0.03335 \\
0.50839 & 0.04812 & 0.50839 & -0.03419 \\
0.48805 & 0.04922 & 0.48805 & -0.03497 \\
0.46772 & 0.05024 & 0.46772 & -0.03569 \\
0.44738 & 0.05118 & 0.44738 & -0.03635 \\
0.42705 & 0.05203 & 0.42705 & -0.03695 \\
0.40671 & 0.0528 & 0.40671 & -0.03749
\end{tabular}


Table B.5 SC 1095 Airfoil Coordinates Continued [111]

\begin{tabular}{|c|c|c|c|}
\hline \multicolumn{2}{|c|}{ Upper Surface } & \multicolumn{2}{|c|}{ Lower Surface } \\
\hline $\mathrm{X} / \mathrm{C}[-]$ & $\mathrm{Y} / \mathrm{C}[-]$ & $\mathrm{X} / \mathrm{C}[-]$ & $\mathrm{Y} / \mathrm{C}[-]$ \\
\hline 0.38638 & 0.05348 & 0.38638 & -0.03797 \\
\hline 0.36604 & 0.05407 & 0.36604 & -0.03839 \\
\hline 0.3457 & 0.05458 & 0.3457 & -0.03874 \\
\hline 0.32537 & 0.05498 & 0.32537 & -0.03903 \\
\hline 0.30503 & 0.05528 & 0.30503 & -0.03924 \\
\hline 0.2847 & 0.05547 & 0.2847 & -0.03937 \\
\hline 0.26945 & 0.05554 & 0.26945 & -0.03941 \\
\hline 0.25419 & 0.05553 & 0.25419 & -0.03941 \\
\hline 0.23894 & 0.05541 & 0.23894 & -0.03935 \\
\hline 0.22369 & 0.05518 & 0.22369 & -0.03923 \\
\hline 0.20844 & 0.0548 & 0.20844 & -0.03903 \\
\hline 0.19319 & 0.05426 & 0.19319 & -0.03876 \\
\hline 0.17794 & 0.05354 & 0.17794 & -0.03841 \\
\hline 0.16268 & 0.05265 & 0.16268 & -0.03795 \\
\hline 0.14743 & 0.05158 & 0.14743 & -0.03737 \\
\hline 0.13218 & 0.05033 & 0.13218 & -0.03666 \\
\hline 0.11693 & 0.04885 & 0.11693 & -0.0358 \\
\hline 0.10168 & 0.04705 & 0.10168 & -0.03476 \\
\hline 0.09151 & 0.04562 & 0.09151 & -0.03397 \\
\hline 0.08134 & 0.04398 & 0.08134 & -0.03304 \\
\hline 0.07117 & 0.04205 & 0.07117 & -0.03191 \\
\hline 0.06101 & 0.03979 & 0.06101 & -0.03048 \\
\hline 0.05084 & 0.03707 & 0.05084 & -0.02862 \\
\hline 0.04271 & 0.03443 & 0.04271 & -0.02669 \\
\hline 0.0366 & 0.03208 & 0.0366 & -0.02494 \\
\hline 0.0305 & 0.02934 & 0.0305 & -0.02293 \\
\hline 0.0244 & 0.02608 & 0.0244 & -0.02066 \\
\hline 0.0183 & 0.0222 & 0.0183 & -0.01798 \\
\hline 0.0122 & 0.01748 & 0.0122 & -0.01453 \\
\hline 0.00915 & 0.01473 & 0.00915 & -0.01236 \\
\hline 0.00661 & 0.01215 & 0.00661 & -0.0102 \\
\hline 0.00407 & 0.00913 & 0.00407 & -0.00757 \\
\hline 0.00203 & 0.00626 & 0.00203 & -0.0051 \\
\hline 0.00081 & 0.00396 & 0.00081 & -0.00322 \\
\hline 0.0001 & 0.00147 & 0.0001 & -0.00112 \\
\hline 0 & 0 & 0 & 0 \\
\hline
\end{tabular}




\section{Appendix C. The Permission to Reproduce Copyrighted Materials}

This appendix comprises the permission attained from the AHS journal publisher allowing the reproduction of the copyrighted materials presented in Chapter 2, section 2.6; Chapter 3, section 3.4; Chapter 4, subsection 4.4.1; and Chapter 5, subsection 5.1.2. 


\title{
ASSIGNMENT OF COPYRIGHT OWNERSHIP
}

AHS International - The Vertical Flight Technical Society

Journal of the American Helicopter Society

I, Khider Al-Jaburi (hereinafter, "the author") as author(s)

(or employer, in the case of "work made for hire") of the article entitled:

(hereinafter "the article") hereby grant and assign to the AHS International (hereinafter, "the publisher") all rights of copyright ownership now and hereafter protected in the article (for U.S. Government employees, to the extent transferable) effective if and when the article is accepted for publication in the Journal of the American Helicopter Society, including, but not limited to, derivation rights, the right to reproduce and distribute the article as part of the issue, and the right to grant or refuse permission to third parties to republish all or part of the article.

Provided, however, that the right to grant or refuse permission to third parties to republish all or part of this article (subject to giving proper credit including the copyright notice) shall also be held by the author.

And further provided, however, that the author reserves the right as author or editor to republish or use all or part of this article in future works, but not limited to lectures, press releases, reviews, or textbooks.

Name: (Print) Khider Al-Jaburi

Signature:

- Author

- Employer (if the paper is the result of "work made for hire")

To ensure that you and AHS International are protected from unauthorized use of copyright material, we request that you (or, in the case of "work made for hire", your employer) sign and return this transfer agreement to AHS International at the email or address listed below. This must be received before your article can be accepted for publication.

\author{
AHS International \\ Attn: Journal Production Manager \\ 2701 Prosperity Ave., Suite 210 \\ Fairfax, VA 22031 \\ Tel. 1-703-684-6777, Fax: 1-703-739-9279 \\ Email: staff@vtol.org
}


11/29/2018_Mail - KhiderAIJaburi@cmail.carleton.ca

FW: Permission to use AHS paper: Volume 63, Number 3, July 2018, pp. 1-14(14).

Julie Gibbs <jmgibbs@vtol.org>

Fri 11/30/2018 12:35 AM

To:Khider Al Jaburi <KhiderAlJaburi@cmail.carleton.ca>;

Khider: thank you for your email and seeking permission to use data from your AHS Journal technical article "Passive Flow Control of Dynamic Stall via Surface-Based Trapped Vortex Generators"

AHS grants you permission to use the information in your thesis but please cite use of the figure etc. accordingly "Passive Flow Control of Dynamics Stall via Surface Based Trapped Vortex Generators" by Khider Al-Jaburi, published in the American Helicopter Society Journal, Volume 63, Number 3, July 2018, pp. 1-14.

Thank you and good luck.

Julie M. Gibbs, Technical Programs Director

AHS - The Vertical Flight Society

Phone: $1-703-684-6777 \times 103$

Toll Free: 1-855-AHS-INTL

jmgibbs@vtol.org

www.vtol.org

Don't miss Forum $75 \star$ The Future of Vertical Flight!

May 13-16, $2019 \star$ Philadelphia, PA, USA

From: Julie Gibbs <jmgibbs@vtol.org>

Sent: Wednesday, November 28, 2018 9:05 AM

To: jmgibbs@vtol.org

Subject: FW: Permission to use AHS paper: Volume 63, Number 3, July 2018, pp. 1-14(14).

Julie M. Gibbs, Technical Programs Director

AHS - The Vertical Flight Society

Phone: 1-703-684-6777 x103

Toll Free: 1-855-AHS-INTL

jmgibbs@vtol.org

www.vtol.org

Don't miss Forum $75 \star$ The Future of Vertical Flight!

May 13-16, $2019 \star$ Philadelphia, PA, USA 
$11 / 29 / 2018$

Mail - KhiderAlJaburi@cmail.carleton.ca

From: Khider Al Jaburi <KhiderAlJaburi@cmail.carleton.ca $>$

Sent: Tuesday, November 27, 2018 6:48 PM

To: staff@vtol.org

Subject: Permission to use AHS paper: Volume 63, Number 3, July 2018, pp. 1-14(14).

Dear JAHS,

I am writing this email to request for permission to use the paper titled "Passive Flow Control of Dynamic Stall via Surface-Based Trapped Vortex Generators" in my PhD thesis. This paper published in Journal of the American Helicopter Society, Volume 63, Number 3, July 2018, pp. 1-14(14). I am the first author of the paper, and I need to reproduce the text, figures, and tables inside my thesis. Readers of the thesis will be explicitly instructed to cite the AHS paper not the thesis regarding any reproduced materials.

Thanks,

Khider Al-Jaburi, B.Sc., M.Sc.

Ph.D. Candidate

Department of Mechanical and Aerospace Engineering

Carleton University

1125 Colonel By Drive

K1S 5B6 Ottawa, ON

Canada

Phone: (613)262-1793

e-mail: khideraljaburi@cmail.carleton.ca 